\title{
Pyridazine N-Oxides as Precursors of Metallocarbenes: Rhodium-Catalyzed Transannulation with Pyrroles
}

\author{
Vinaykumar Kanchupalli, Desna Joseph and Sreenivas Katukojvala* \\ Department of Chemistry, Indian Institute of Science Education \& Research, Bhopal, MP, India 462066
}

\section{Supporting Information}

\section{Contents :}

$\begin{array}{ll}\text { 1. General methods } & \text { S2 }\end{array}$

2. Starting materials $\quad$ S2

$\begin{array}{ll}\text { a) Preparation of pyridazines s1-s19 } & \text { S2 }\end{array}$

$\begin{array}{ll}\text { b) Preparation of pyridazine } N \text {-oxides 9a-t } & \text { S10 }\end{array}$

$\begin{array}{lr}\text { c) Preparation of pyridazinium salts } 6 & \text { S18 }\end{array}$

$\begin{array}{lr}\text { d) Preparation of substituted pyrroles } & \text { S19 }\end{array}$

3. Optimization of transannulation reaction $\quad$ S19

4. Scope of the transannulation towards alkylindoles $\quad$ S22

5. Scope of the transannulation towards 7-arylindoles $\quad$ S25

6. Transannulation towards 7-vinylindoles $\quad$ S33

7. Synthetic applications of transannulation $\quad$ S35

$\begin{array}{ll}\text { 8. Characterization of the intermediate } \mathbf{1 5 b} & \text { S38 }\end{array}$

9. XRD crystal data of 10fc $\quad$ S40

$\begin{array}{ll}\text { 10. References } & \text { S41 }\end{array}$

$\begin{array}{ll}\text { 11. NMR spectra } & \text { S42 }\end{array}$ 


\section{General Methods:}

All the reactions were performed in an oven-dried glassware under argon atmosphere. Solvents were dried using standard methods. Dimethoxy ethane, dichloromethane were distilled over calcium hydride. Unless otherwise stated, all the commercial reagents were used as received. Progress of the reaction was monitored by thin layer chromatography (Merck Silica gel 60 F254, $0.25 \mathrm{~nm}$, precoated plates on alumina). Column chromatographic purifications were performed on Merck silica gel (100-200 mesh). Melting points were recorded on a digital melting point apparatus and are uncorrected.

Spectroscopic characterizations were carried out at the Central Instrumentation Facility (CIF), Indian Institute of Science Education and Research (IISER) Bhopal. ${ }^{1} \mathrm{H}-\mathrm{NMR}$ spectra were recorded on Bruker Avance III FT-NMR spectrometers at $400 \mathrm{MHz}, 500 \mathrm{MHz}$ or $700 \mathrm{MHz}$ and ${ }^{13} \mathrm{C}-\mathrm{NMR}$ spectra were recorded at $101 \mathrm{MHz}, 126 \mathrm{MHz}$ or $176 \mathrm{MHz} .{ }^{1} \mathrm{H}-\mathrm{NMR}$ chemical shifts are reported in ppm relative to the TMS $(\delta=0)$ or $\mathrm{CDCl}_{3}$ signal $(\delta=7.26)$ and are abbreviated as follows: s (singlet), d (doublet), $\mathrm{t}$ (triplet), q (quartet), m (multiplet), br (broad). ${ }^{13} \mathrm{C}-\mathrm{NMR}$ chemical shifts are reported in ppm relative to the residual $\mathrm{CDCl}_{3}$ signal $(\delta=77.16)$. IR spectra were recorded on a Perkin Elmer FT-IR spectrometer. HRMS data was obtained on a Bruker microTOF-QII or Agilent 5975C high resolution mass spectrometers.

\section{Starting materials:}

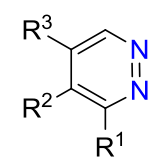

s1 - s19

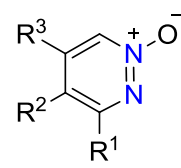

9a-t

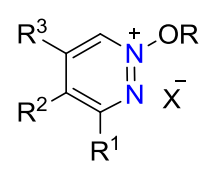

6a-t

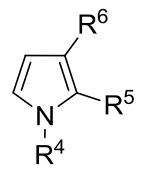

sa-i

\section{a) Preparation of pyridazines s1-s19}

Pyridazines s1, s2 and $\mathbf{s 4}$ were purchased from Sigma Aldrich. 3-Phenethylpyridazine s3 was prepared by following the literature procedure. ${ }^{1}$ 


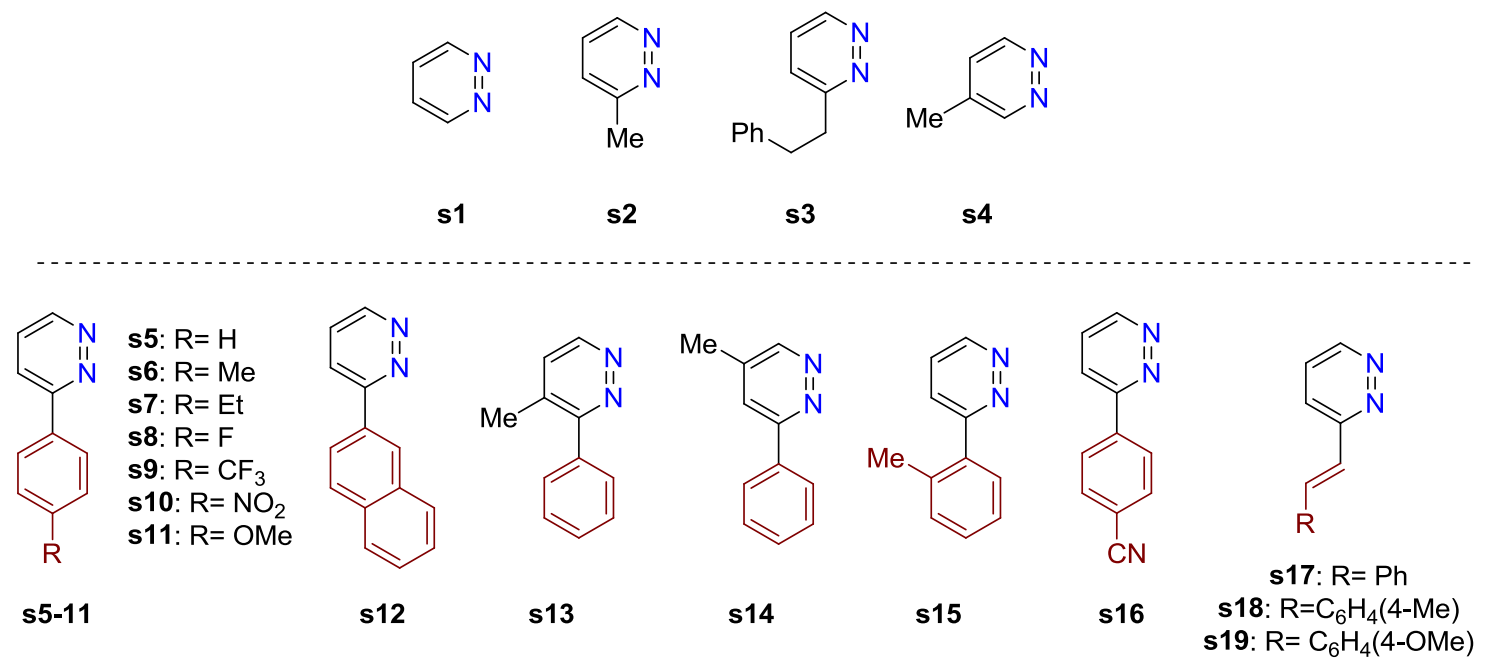

3-Arylpyridazines s5-s12, s15 and s16 were prepared from 3-chloropyridazine ${ }^{2}$ according to the general procedure $A$. The disubstituted pyridazines $\mathbf{s 1 3}$ and $\mathbf{s 1 4}$ were prepared from commercially available 3,6-dichloro-4-methylpyridazine according to the general procedure $\mathbf{B}$. 3-Vinylpyridazines s17-s19 were prepared by the known procedures. ${ }^{3}$

General procedure A: Preparation of 3-arylpyridazines s5-s12, s15 and s16

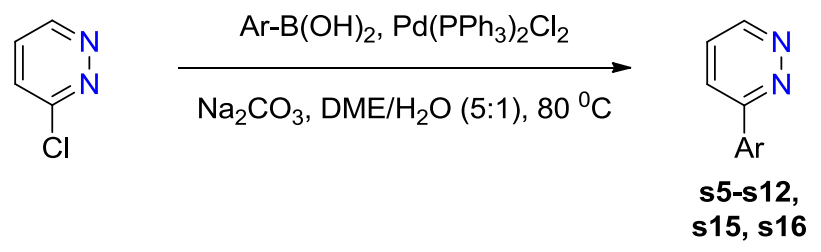

In a flame-dried round bottom flask $\mathrm{Pd}\left(\mathrm{PPh}_{3}\right)_{2} \mathrm{Cl}_{2}$ (0.4 mmol, 0.1 equiv), $\mathrm{Na}_{2} \mathrm{CO}_{3}$ (22 mmol, 5 equiv) and dry dimethoxy ethane $(10 \mathrm{ml})$ were taken. The solution was degassed with argon for $10 \mathrm{~min}$ followed by addition of aryl boronic acid (4.8 mmol, 1.1 equiv) and 3-chloropyridazine (4.3 mmol, 1 equiv). After $2 \mathrm{~min}$ water $(2 \mathrm{ml})$ was added and the reaction continued for $12 \mathrm{~h}$ at $80{ }^{\circ} \mathrm{C}$ under inert atmosphere. The reaction mixture was cooled to room temperature and extracted with DCM $(50 \mathrm{ml})$ and water $(10 \mathrm{ml})$. The organic layer was washed with brine $(10$ $\mathrm{ml}$ ), dried over anhydrous $\mathrm{Na}_{2} \mathrm{SO}_{4}$ and concentrated. Purification of the crude product by silica gel column chromatography afforded 3-arylpyridazine. 


\section{General procedure B: Preparation of disubstituted pyridazines s13 and s14}<smiles>Cc1cc(Cl)nnc1Cl</smiles>

$$
\underset{\mathrm{Na}_{2} \mathrm{CO}_{3}, \mathrm{DME} / \mathrm{H}_{2} \mathrm{O}(5: 1), 80^{\circ} \mathrm{C}}{\stackrel{\mathrm{Ar}}{\mathrm{O}})_{2}, \mathrm{Pd}\left(\mathrm{PPh}_{3}\right)_{2} \mathrm{Cl}_{2}}
$$<smiles>Cc1cc(Cl)nnc1Br</smiles>

s13a<smiles>Cc1cc(Br)nnc1Cl</smiles>

s14a

1:1 mixture

Step I: In a flame-dried round bottom flask $\mathrm{Pd}\left(\mathrm{PPh}_{3}\right)_{2} \mathrm{Cl}_{2}$ (0.3 mmol, 0.1 equiv), $\mathrm{Na}_{2} \mathrm{CO}_{3}(15$ mmol, 5 equiv) and dry dimethoxy ethane $(10 \mathrm{ml})$ were taken. The solution was degassed with argon for $10 \mathrm{~min}$ followed by addition of aryl boronic acid (3.3 mmol, 1.1 equiv) and 3,6dichloro-4-methylpyridazine (3.0 mmol, 1 equiv). After 2 min water $(2 \mathrm{ml})$ was added and the reaction was continued for $12 \mathrm{~h}$ at $80{ }^{\circ} \mathrm{C}$ under inert atmosphere. The reaction mixture was cooled to room temperature and extracted with DCM $(50 \mathrm{ml})$ and $\mathrm{H}_{2} \mathrm{O}(10 \mathrm{ml})$. The organic layer was washed with brine $(10 \mathrm{ml})$, dried over anhydrous $\mathrm{Na}_{2} \mathrm{SO}_{4}$ and concentrated. Purification of the crude product by silica gel column chromatography afforded a 1:1 mixture of s13a and s14a.

Step II: Hydrogenation of s13a and s14a

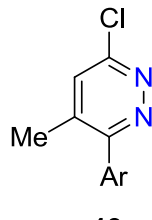

(or)<smiles>Cc1cc(Br)nnc1Cl</smiles>

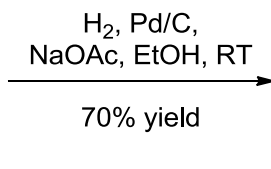<smiles>Cc1ccnnc1Br</smiles>

(or)

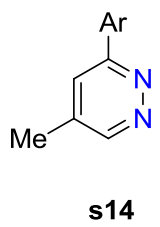

To a solution of $\mathbf{s 1 3 a}$ or $\mathbf{s 1 4 a}(0.63 \mathrm{mmol}, 1$ equiv $)$ in ethanol $(5 \mathrm{ml})$ was added $\mathrm{NaOAc}(1.27$ mmol, 2 equiv) and $10 \% \mathrm{Pd} / \mathrm{C}(0.06 \mathrm{mmol}, 0.1$ equiv). The contents were stirred under hydrogen atmosphere (with hydrogen balloon) at rt. After completion (monitored by TLC) of the reaction $(5 \mathrm{~h})$, the reaction mixure was filtered through a celite pad and concentrated under reduced pressure. The residue was purified by a silca gel column chromatography using ethyl acetate/hexanes as the eluent to give $\mathbf{s 1 3}$ or $\mathbf{s 1 4}$.

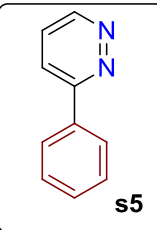


3-Phenylpyridazine (s5): ${ }^{4}$ Prepared by following General procedure A. Obtained as a white solid $(0.402 \mathrm{~g}$, Yield $=60 \%) ; \mathrm{R}_{\mathrm{f}}=0.5$ (ethyl acetate/hexane :50:50); ${ }^{1} \mathrm{H}$ NMR (400 MHz, $\left.\mathrm{CDCl}_{3}\right) \delta 9.15(\mathrm{~d}, J=2.9 \mathrm{~Hz}, 1 \mathrm{H}), 8.07(\mathrm{~d}, J=7.5 \mathrm{~Hz}, 2 \mathrm{H}), 7.85(\mathrm{~d}, J=8.6 \mathrm{~Hz}, 1 \mathrm{H}), 7.61-7.42$ $(\mathrm{m}, 4 \mathrm{H}) ;{ }^{13} \mathrm{C} \mathrm{NMR}\left(101 \mathrm{MHz}, \mathrm{CDCl}_{3}\right) \delta 159.6,150.1,136.5,130.2,129.1,127.2,126 ., 124.0$.

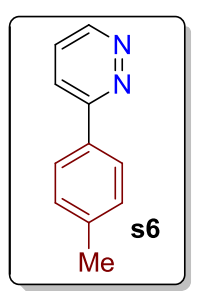

3-(p-Tolyl)pyridazine (s6): ${ }^{5}$ Prepared by following General procedure A. Obtained as a white solid $(0.402 \mathrm{~g}$, Yield $=55 \%) ; \mathrm{R}_{\mathrm{f}}=0.5$ (ethyl acetate/hexane : 50:50); ${ }^{1} \mathrm{H} \mathrm{NMR}(500 \mathrm{MHz}$, $\left.\mathrm{CDCl}_{3}\right) \delta 9.13-9.11(\mathrm{~m}, 1 \mathrm{H}), 7.97(\mathrm{~d}, J=8.2 \mathrm{~Hz}, 2 \mathrm{H}), 7.84(\mathrm{dd}, J=8.6,1.6 \mathrm{~Hz}, 1 \mathrm{H}), 7.52(\mathrm{dd}$, $J=8.6,4.9 \mathrm{~Hz}, 1 \mathrm{H}), 7.34(\mathrm{dd}, J=8.0,0.5 \mathrm{~Hz}, 2 \mathrm{H}), 2.43(\mathrm{~s}, 3 \mathrm{H}) ;{ }^{13} \mathrm{C} \mathrm{NMR}\left(126 \mathrm{MHz}, \mathrm{CDCl}_{3}\right) \delta$ $159.6,149.8,140.6,133.5,130.0,127.1,127.1,124.0,21.5$.

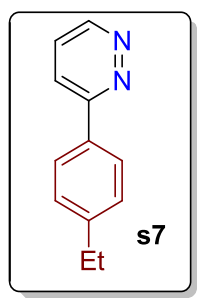

3-(4-Ethylphenyl)pyridazine (s7): Prepared by following General procedure A. Obtained as a white solid (0.411 g, Yield = 52\%); m.p. $=86-88{ }^{\circ} \mathrm{C} ; \mathrm{R}_{\mathrm{f}}=0.5$ (ethyl acetate/hexane : 50:50); ${ }^{1} \mathrm{H}$ NMR $\left(500 \mathrm{MHz}, \mathrm{CDCl}_{3}\right) \delta 9.12(\mathrm{dd}, J=4.9,1.6 \mathrm{~Hz}, 1 \mathrm{H}), 8.02-7.99(\mathrm{~m}, 2 \mathrm{H}), 7.83(\mathrm{dd}, J=8.6$, $1.6 \mathrm{~Hz}, 1 \mathrm{H}), 7.50(\mathrm{dd}, J=8.6,4.9 \mathrm{~Hz}, 1 \mathrm{H}), 7.35(\mathrm{~d}, J=8.4 \mathrm{~Hz}, 2 \mathrm{H}), 2.72(\mathrm{q}, J=7.6 \mathrm{~Hz}, 2 \mathrm{H})$, $1.28(\mathrm{t}, J=7.6 \mathrm{~Hz}, 3 \mathrm{H}) ;{ }^{13} \mathrm{C} \mathrm{NMR}\left(126 \mathrm{MHz}, \mathrm{CDCl}_{3}\right) \delta 159.6,149.9,146.7,133.9,128.7,127.2$, 126.8, 123.7, 28.8, 15.5; IR (neat): 3065, 2965, 2924, 1650, 1548, 837, $765 \mathrm{~cm}^{-1}$; HRMS (ESI) $m / z$ calc. for $\mathrm{C}_{12} \mathrm{H}_{12} \mathrm{~N}_{2}(\mathrm{M}+\mathrm{H})^{+}$185.1073, found 185.1070 .

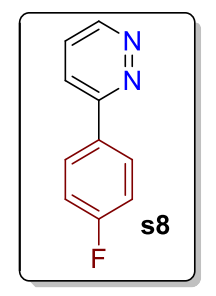

3-(4-Fluorophenyl)pyridazine (s8): ${ }^{6}$ Prepared by following General procedure A. Obtained as a white solid $(0.374 \mathrm{~g}$, Yield $=50 \%) ; \mathrm{R}_{\mathrm{f}}=0.5$ (ethyl acetate/hexane $\left.: 50: 50\right) ;{ }^{1} \mathrm{H}$ NMR (400 
$\left.\mathrm{MHz}, \mathrm{CDCl}_{3}\right) \delta 9.13(\mathrm{dd}, J=4.8,1.3 \mathrm{~Hz}, 1 \mathrm{H}), 8.06(\mathrm{dd}, J=8.8,5.3 \mathrm{~Hz}, 2 \mathrm{H}), 7.81(\mathrm{dd}, J=8.6$, $1.4 \mathrm{~Hz}, 1 \mathrm{H}), 7.52(\mathrm{dd}, J=8.6,4.9 \mathrm{~Hz}, 1 \mathrm{H}), 7.19(\mathrm{t}, J=8.6 \mathrm{~Hz}, 2 \mathrm{H}) ;{ }^{13} \mathrm{C}$ NMR $(101 \mathrm{MHz}$, $\left.\mathrm{CDCl}_{3}\right) \delta 164.3(\mathrm{~d}, J=250.4 \mathrm{~Hz}), 158.9,150.0,132.6(\mathrm{~d}, J=3.2 \mathrm{~Hz}), 129.2(\mathrm{~d}, J=8.5 \mathrm{~Hz})$, $126.9,123.6,116.2(\mathrm{~d}, J=21.8 \mathrm{~Hz})$.

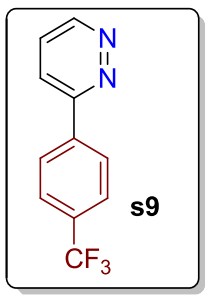

3-(4-(Trifluoromethyl)phenyl)pyridazine (s9): Prepared by following General procedure A. Obtained as a white solid $(0.481 \mathrm{~g}$, Yield $=50 \%)$; m.p. $=136-138{ }^{\circ} \mathrm{C} ; \mathrm{R}_{\mathrm{f}}=0.5$ (ethyl acetate/hexane : 50:50); ${ }^{1} \mathrm{H}$ NMR $\left(400 \mathrm{MHz}, \mathrm{CDCl}_{3}\right) \delta 9.21(\mathrm{dd}, J=4.9,1.4 \mathrm{~Hz}, 1 \mathrm{H}), 8.20(\mathrm{~d}, J$ $=8.2 \mathrm{~Hz}, 2 \mathrm{H}), 7.90(\mathrm{dd}, J=8.6,1.4 \mathrm{~Hz}, 1 \mathrm{H}), 7.78(\mathrm{~d}, J=8.2 \mathrm{~Hz}, 2 \mathrm{H}), 7.59(\mathrm{dd}, J=8.6,4.9 \mathrm{~Hz}$, $1 \mathrm{H}) ;{ }^{13} \mathrm{C}$ NMR $\left(101 \mathrm{MHz}, \mathrm{CDCl}_{3}\right) \delta 158.3,150.7,139.8,132.1$ (q, $\left.J=32.7 \mathrm{~Hz}\right), 127.6(2 \mathrm{C})$, 127.1, 126.1 (q, $J=3.7 \mathrm{~Hz}$ ), 124.2; IR (neat): 3035, 1640, 1443, 1094, 835, $735 \mathrm{~cm}^{-1}$; HRMS (ESI) $m / z$ calc. for $\mathrm{C}_{11} \mathrm{H}_{7} \mathrm{~F}_{3} \mathrm{~N}_{2}(\mathrm{M}+\mathrm{H})^{+} 225.0634$, found 225.0658 .

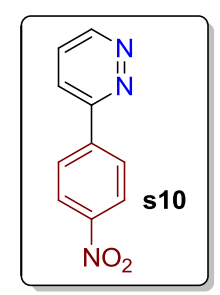

3-(4-Nitrophenyl)pyridazine (s10): Prepared by following General procedure A. Obtained as a white solid $(0.501 \mathrm{~g}$, Yield $=58 \%) ; \mathrm{m} . \mathrm{p} .=136-138{ }^{\circ} \mathrm{C} ; \mathrm{R}_{\mathrm{f}}=0.45$ (ethyl acetate/hexane:50:50); ${ }^{1} \mathrm{H}$ NMR $\left(400 \mathrm{MHz}, \mathrm{CDCl}_{3}\right) \delta 9.26(\mathrm{dd}, J=4.9,1.3 \mathrm{~Hz}, 1 \mathrm{H}), 8.39(\mathrm{~d}, J=8.8 \mathrm{~Hz}, 2 \mathrm{H}), 8.28(\mathrm{~d}, J$ $=8.9 \mathrm{~Hz}, 2 \mathrm{H}), 7.95(\mathrm{dd}, J=8.6,1.4 \mathrm{~Hz}, 1 \mathrm{H}), 7.64(\mathrm{dd}, J=8.6,5.0 \mathrm{~Hz}, 1 \mathrm{H}) ;{ }^{13} \mathrm{C} \mathrm{NMR}(101$ $\left.\mathrm{MHz}, \mathrm{CDCl}_{3}\right) \delta 157.6,150.9,149.1,142.4,128.2,127.2,124.4,124.4$; IR (neat): 3066, 2922, 2843, 1501, 1350, 1294, 1110, $813 \mathrm{~cm}^{-1}$; HRMS (ESI) $\mathrm{m} / z$ calc. for $\mathrm{C}_{10} \mathrm{H}_{7} \mathrm{~N}_{3} \mathrm{O}_{2}(\mathrm{M}+\mathrm{H})^{+}$ 202.0611, found 202.0640.

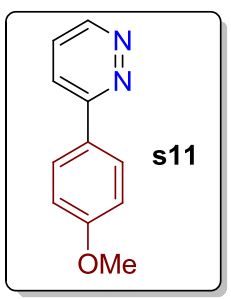


3-(4-Methoxyphenyl)pyridazine (s11): ${ }^{6}$ Prepared by following General procedure A. Obtained as a white solid $(0.480 \mathrm{~g}$, Yield $=60 \%) ; \mathrm{R}_{\mathrm{f}}=0.5$ (ethyl acetate/hexane : 50:50); ${ }^{1} \mathrm{H}$ NMR $\left(400 \mathrm{MHz}, \mathrm{CDCl}_{3}\right) \delta 9.05(\mathrm{dd}, J=4.9,1.6 \mathrm{~Hz}, 1 \mathrm{H}), 8.04-8.00(\mathrm{~m}, 2 \mathrm{H}), 7.76(\mathrm{dd}, J=8.7$, $1.6 \mathrm{~Hz}, 1 \mathrm{H}), 7.45(\mathrm{dd}, J=8.7,4.9 \mathrm{~Hz}, 1 \mathrm{H}), 7.04-6.98(\mathrm{~m}, 2 \mathrm{H}), 3.84(\mathrm{~s}, 3 \mathrm{H}) ;{ }^{13} \mathrm{C}$ NMR $(101$ $\left.\mathrm{MHz}, \mathrm{CDCl}_{3}\right) \delta 161.4,159.0,149.5,128.8,128.5,126.8,123.2,114.5,55.4$.

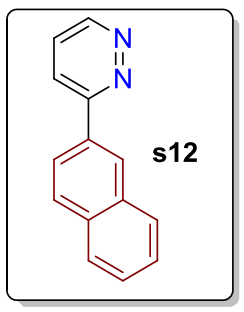

3-(Naphthalen-2-yl)pyridazine (s12): ${ }^{6}$ Prepared by following General procedure A. Obtained as a white solid $(0.513 \mathrm{~g}$, Yield $=58 \%) ; \mathrm{R}_{\mathrm{f}}=0.5$ (ethyl acetate/hexane : 50:50); ${ }^{1} \mathrm{H}$ NMR (400 $\left.\mathrm{MHz} \mathrm{CDCl}_{3}\right) \delta 9.19(\mathrm{~d}, J=4.4 \mathrm{~Hz}, 1 \mathrm{H}), 8.56(\mathrm{~s}, 1 \mathrm{H}), 8.27-8.20(\mathrm{~m}, 1 \mathrm{H}), 8.05-7.93(\mathrm{~m}, 3 \mathrm{H})$, $7.94-7.87(\mathrm{~m}, 1 \mathrm{H}), 7.62-7.50(\mathrm{~m}, 3 \mathrm{H}) ;{ }^{13} \mathrm{C} \mathrm{NMR}\left(101 \mathrm{MHz}, \mathrm{CDCl}_{3}\right) \delta$ 159.6, 150.1, 134.3, $133.8,133.5,129.0,129.0,127.9,127.3,127.2,126.9,126.8,124.3,124.2$.

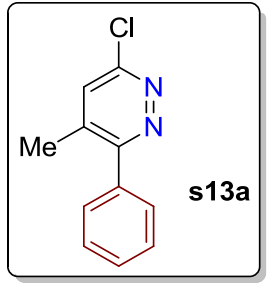

6-Chloro-4-methyl-3-phenylpyridazine (s13a): Prepared by following General procedure B. Obtained as a white solid (0.183 g, Yield $=30 \%$ ); m.p. $=142-144{ }^{\circ} \mathrm{C} ; \mathrm{R}_{\mathrm{f}}=0.5$ (ethyl acetate/hexane: 20:80); ${ }^{1} \mathrm{H}$ NMR (400 MHz, $\left.\mathrm{CDCl}_{3}\right) \delta 7.57-7.46(\mathrm{~m}, 5 \mathrm{H}), 7.42(\mathrm{~s}, 1 \mathrm{H}), 2.36(\mathrm{~s}$, $3 \mathrm{H}) ;{ }^{13} \mathrm{C} \mathrm{NMR}\left(101 \mathrm{MHz}, \mathrm{CDCl}_{3}\right) \delta 161.7,155.3,139.1,136.0,129.4,129.2,129.2,128.7,19.8$; IR (neat): 3054, 2963, 2925, 1589, 1471, 1093, 835, $740 \mathrm{~cm}^{-1}$; HRMS (ESI) m/z calc. for $\mathrm{C}_{11} \mathrm{H}_{9} \mathrm{ClN}_{2}(\mathrm{M}+\mathrm{Na})^{+}$227.0346, found 227.0359.

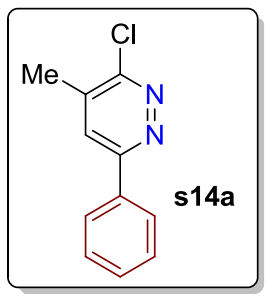

3-Chloro-4-methyl-6-phenylpyridazine (s14a): Prepared by following General procedure B. Obtained as a white solid $\left(0.214 \mathrm{~g}\right.$, Yield $=35 \%$ ); m.p. $=106-108{ }^{\circ} \mathrm{C} ; \mathrm{R}_{\mathrm{f}}=0.46$ (ethyl 
acetate/hexane: 20:80); ${ }^{1} \mathrm{H}$ NMR $\left(400 \mathrm{MHz}, \mathrm{CDCl}_{3}\right) \delta 8.05-7.99(\mathrm{~m}, 2 \mathrm{H}), 7.70(\mathrm{~d}, J=0.9 \mathrm{~Hz}$, 1H), $7.54-7.48(\mathrm{~m}, 3 \mathrm{H}), 2.47(\mathrm{~d}, J=0.8 \mathrm{~Hz}, 3 \mathrm{H}) ;{ }^{13} \mathrm{C} \mathrm{NMR}\left(101 \mathrm{MHz}, \mathrm{CDCl}_{3}\right) \delta 158.9,157.0$, 138.2, 135.4, 130.4, 129.2, 127.2, 126.6, 19.6; IR (neat): 3035, 2963, 2925, 1668, $1095835 \mathrm{~cm}^{-1}$; HRMS (ESI) $m / z$ calc. for $\mathrm{C}_{11} \mathrm{H}_{9} \mathrm{ClN}_{2}(\mathrm{M}+\mathrm{Na})^{+} 227.0346$, found 227.0359 .

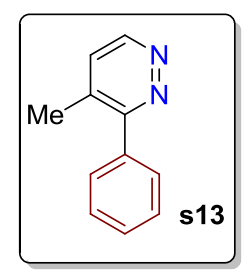

4-Methyl-3-phenylpyridazine (s13): Prepared by following General procedure B. Obtained as a colourless liquid $(0.357 \mathrm{~g}$, Yield $=70 \%) ; \mathrm{R}_{\mathrm{f}}=0.45$ (ethyl acetate/hexane:50:50); ${ }^{1} \mathrm{H}$ NMR (400 $\left.\mathrm{MHz}, \mathrm{CDCl}_{3}\right) \delta 8.99(\mathrm{~d}, J=5.1 \mathrm{~Hz}, 1 \mathrm{H}), 7.57-7.52(\mathrm{~m}, 2 \mathrm{H}), 7.50-7.41(\mathrm{~m}, 3 \mathrm{H}), 7.34(\mathrm{dd}, J=$ 5.1, $0.5 \mathrm{~Hz}, 1 \mathrm{H}), 2.34(\mathrm{~s}, 3 \mathrm{H}) ;{ }^{13} \mathrm{C} \mathrm{NMR}\left(101 \mathrm{MHz}, \mathrm{CDCl}_{3}\right) \delta 162.4,149.8,137.1,136.2,129.2$, 128.9, 128.5, 128.2, 19.7; IR (neat): 3054, 2963, 2926, 1601, 1107, 840, $795 \mathrm{~cm}^{-1}$; HRMS (ESI) $m / z$ calc. for $\mathrm{C}_{11} \mathrm{H}_{10} \mathrm{~N}_{2}(\mathrm{M}+\mathrm{H})^{+} 171.0917$, found 171.0934 .

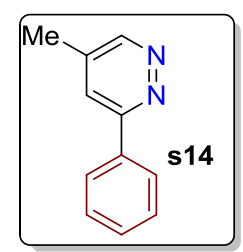

5-Methyl-3-phenylpyridazine (s14): ${ }^{4}$ Prepared by following General procedure B. Obtained as a white solid $(0.357 \mathrm{~g}$, Yield $=70 \%) ; \mathrm{R}_{\mathrm{f}}=0.5$ (ethyl acetate/hexane : 50:50); ${ }^{1} \mathrm{H}$ NMR (400 $\left.\mathrm{MHz}, \mathrm{CDCl}_{3}\right) \delta 8.97(\mathrm{~s}, 1 \mathrm{H}), 8.03(\mathrm{dd}, J=7.1,1.0 \mathrm{~Hz}, 2 \mathrm{H}), 7.62(\mathrm{~s}, 1 \mathrm{H}), 7.53-7.42(\mathrm{~m}, 3 \mathrm{H})$, $2.39(\mathrm{~s}, 3 \mathrm{H}) ;{ }^{13} \mathrm{C}$ NMR $\left(101 \mathrm{MHz}, \mathrm{CDCl}_{3}\right) \delta 158.9,151.6,138.0,136.5,130.0,129.0,127.2$, 124.4, 18.6.

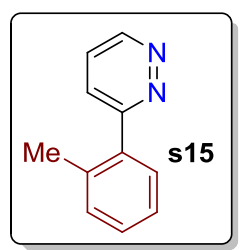

3-(o-Tolyl)pyridazine (s15): ${ }^{7}$ Prepared by following General procedure A. Obtained as a white solid $(0.402 \mathrm{~g}$, Yield $=55 \%) ; \mathrm{m} . \mathrm{p} .=86-88{ }^{\circ} \mathrm{C} ; \mathrm{R}_{\mathrm{f}}=0.5$ (ethyl acetate/hexane : 50:50); ${ }^{1} \mathrm{H}$ NMR $\left(400 \mathrm{MHz}, \mathrm{CDCl}_{3}\right) \delta 9.18(\mathrm{dd}, J=4.7,1.9 \mathrm{~Hz}, 1 \mathrm{H}), 7.59-7.51(\mathrm{~m}, 2 \mathrm{H}), 7.45-7.29(\mathrm{~m}, 4 \mathrm{H}), 2.38$ 
(s, 3H) ${ }^{13} \mathrm{C}$ NMR $\left(101 \mathrm{MHz}, \mathrm{CDCl}_{3}\right) \delta$ 162.4, 149.7, 137.3, 136.3, 131.1, 129.9, 129.4, 127.4, $126.3,126.2,20.4$.

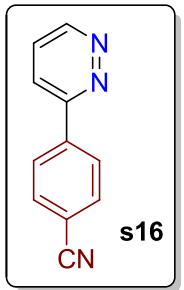

4-(Pyridazin-3-yl)benzonitrile (s16): Prepared by following General procedure A. Obtained as a white color solid $(0.350 \mathrm{~g}$, Yield $=45 \%) ; \mathrm{m} . \mathrm{p} .=146-148{ }^{\circ} \mathrm{C} ; \mathrm{R}_{\mathrm{f}}=0.35$ (ethyl acetate/hexane:50:50); ${ }^{1} \mathrm{H}$ NMR (500 MHz, $\mathrm{CDCl}_{3}$ ) $\delta 9.22(\mathrm{dd}, J=4.9,1.6 \mathrm{~Hz}, 1 \mathrm{H}$ ), $8.22-8.19$ $(\mathrm{m}, 2 \mathrm{H}), 7.90(\mathrm{dd}, J=8.6,1.6 \mathrm{~Hz}, 1 \mathrm{H}), 7.84-7.79(\mathrm{~m}, 2 \mathrm{H}), 7.61(\mathrm{dd}, J=8.6,5.0 \mathrm{~Hz}, 1 \mathrm{H}) ;{ }^{13} \mathrm{C}$ NMR $\left(126 \mathrm{MHz}, \mathrm{CDCl}_{3}\right) \delta 157.8,150.8,140.58,132.9,127.8,127.2,124.2,118.5,113.9$; IR (neat): $3055,2985,2926,2223,1590,1430,1269,1176,987,810 \mathrm{~cm}^{-1}$; HRMS (ESI) $\mathrm{m} / z$ calc. for $\mathrm{C}_{11} \mathrm{H}_{7} \mathrm{~N}_{3}(\mathrm{M}+\mathrm{H})^{+}$182.0713, found 182.0734 .

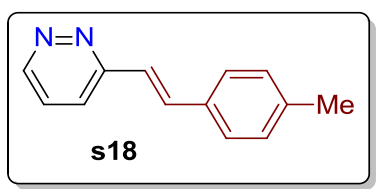

(E)-3-(4-Methylstyryl)pyridazine (s18): Prepared from 3-methylpyridazine s2 according to the literature procedure. ${ }^{2}$ Obtained as a brown solid $(0.357 \mathrm{~g}$, Yield $=70 \%)$; m.p. $=130-134{ }^{\circ} \mathrm{C} ; \mathrm{R}_{\mathrm{f}}$ $=0.6$ (ethyl acetate/hexane:50:50); ${ }^{1} \mathrm{H}$ NMR $\left(400 \mathrm{MHz}, \mathrm{CDCl}_{3}\right) \delta 9.01(\mathrm{dd}, J=4.8,1.5 \mathrm{~Hz}, 1 \mathrm{H})$, $7.64(\mathrm{~d}, J=16.4 \mathrm{~Hz}, 1 \mathrm{H}), 7.59(\mathrm{dd}, J=8.6,1.4 \mathrm{~Hz}, 1 \mathrm{H}), 7.48(\mathrm{~d}, J=8.0 \mathrm{~Hz}, 2 \mathrm{H}), 7.40(\mathrm{dd}, J=$ 8.6, 4.9 Hz, 1H), 7.29 (d, $J=16.4 \mathrm{~Hz}, 1 \mathrm{H}), 7.19(\mathrm{~d}, J=7.9 \mathrm{~Hz}, 2 \mathrm{H}), 2.36(\mathrm{~s}, 3 \mathrm{H}) ;{ }^{13} \mathrm{C}$ NMR $(101$ $\left.\mathrm{MHz}, \mathrm{CDCl}_{3}\right) \delta 158.5,149.6,139.3,135.2,133.2,129.7,127.4,126.5,124.2,123.9,21.5 ; \mathrm{IR}$ (neat): $3043,2916,2858,1635,1575,1431,1255,817 \mathrm{~cm}^{-1}$; HRMS (ESI) $\mathrm{m} / z$ calc. for $\mathrm{C}_{13} \mathrm{H}_{12} \mathrm{~N}_{2}$ $(\mathrm{M}+\mathrm{H})^{+}$197.1073, found 197.1098.

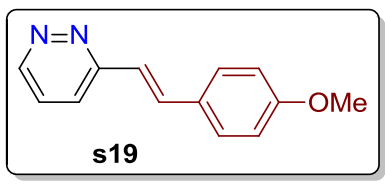

(E)-3-(4-Methoxystyryl)pyridazine (s19): Prepared from 3-methylpyridazine s2 according to the literature procedure. ${ }^{2}$ Obtained as a brown solid $(0.359 \mathrm{~g}$, Yield $=65 \%) ; \mathrm{m} . \mathrm{p} .=116-118{ }^{\circ} \mathrm{C}$; $\mathrm{R}_{\mathrm{f}}=0.5$ (ethyl acetate/hexane:50:50); ${ }^{1} \mathrm{H}$ NMR $\left(400 \mathrm{MHz}, \mathrm{CDCl}_{3}\right) \delta 9.01$ (dd, $J=4.8,1.5 \mathrm{~Hz}$, $1 \mathrm{H}), 7.64(\mathrm{~d}, J=16.4 \mathrm{~Hz}, 1 \mathrm{H}), 7.59(\mathrm{dd}, J=8.6,1.5 \mathrm{~Hz}, 1 \mathrm{H}), 7.55(\mathrm{~d}, J=8.7 \mathrm{~Hz}, 2 \mathrm{H}), 7.41(\mathrm{dd}$, 
$J=8.6,4.9 \mathrm{~Hz}, 1 \mathrm{H}), 7.21(\mathrm{~d}, J=16.4 \mathrm{~Hz}, 1 \mathrm{H}), 6.93(\mathrm{~d}, J=8.7 \mathrm{~Hz}, 2 \mathrm{H}), 3.84(\mathrm{~s}, 3 \mathrm{H}) ;{ }^{13} \mathrm{C} \mathrm{NMR}$ $\left(101 \mathrm{MHz}, \mathrm{CDCl}_{3}\right) \delta 160.6,158.7,149.5,134.9,128.9,128.8,126.5,123.8,123.0,114.5,55.5$; IR (neat): 3090, 2910, 2835, 1645, 1604, 1510, 1253, 1028, $795 \mathrm{~cm}^{-1}$; HRMS (ESI) $\mathrm{m} / z$ calc. for $\mathrm{C}_{13} \mathrm{H}_{12} \mathrm{~N}_{2} \mathrm{O}(\mathrm{M}+\mathrm{H})^{+} 213.1022$, found 213.1038.

\section{b) Preparation of pyridazine $N$-oxides 9 a- $t$}

Pyridazine $N$-oxides 9a-t were prepared from s1-s19 according to the following general procedure $\mathbf{C}$.
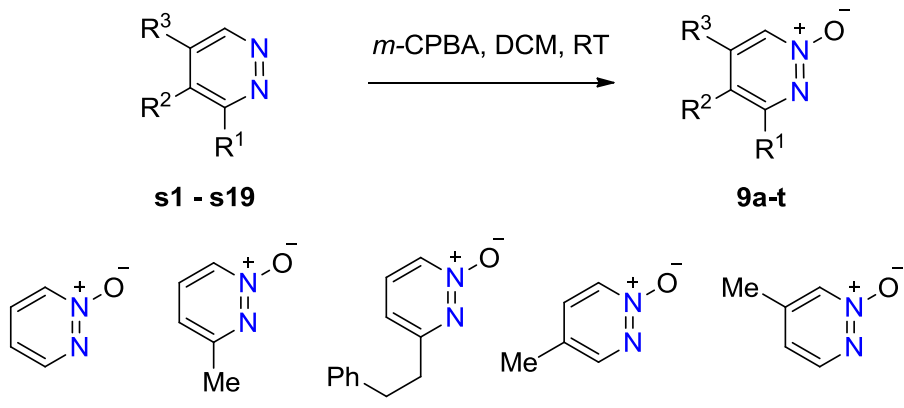

$9 a$

$9 b$

9c

9d

$9 e$

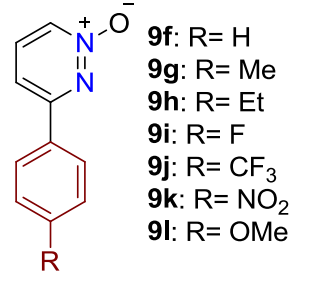

9f-I

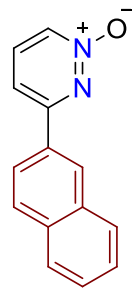

$9 \mathrm{~m}$

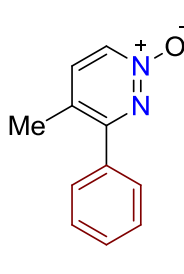

$9 n$<smiles>Cc1cc(-c2ccccc2)n[n+]([O-])c1</smiles>

90<smiles>N#Cc1ccc(-c2ccc[n+]([O-])n2)cc1</smiles>

$9 q$ 9s: $\mathrm{R}=\mathrm{C}_{6} \mathrm{H}_{4}(4-\mathrm{Me})$

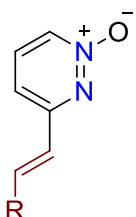

9s: $\mathrm{R}=\mathrm{C}_{6} \mathrm{H}_{4}(4-\mathrm{Me})$
9t: $\mathrm{R}=\mathrm{C}_{6} \mathrm{H}_{4}(4-\mathrm{OMe})$

\section{General procedure C:}

To a solution of pyridazine s1-s19 ( $1 \mathrm{mmol}, 1$ equiv) in DCM at rt was slowly added $m$-CPBA ( $1.5 \mathrm{mmol}, 1.5$ equiv) and continued stirring for $1-2 \mathrm{~h}$ (as judged by TLC). The crude reaction mixture was directly purified by a silica gel flash column chromatography using ethyl acetate petroleum ether or methanol-DCM as the eluent.

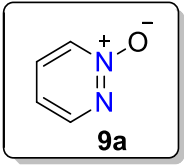


Pyridazine 1-oxide (9a): ${ }^{7}$ Prepared by following General procedure C. Obtained as a clear liquid $(0.094 \mathrm{~g}$, Yield $=98 \%) ; \mathrm{R}_{\mathrm{f}}=0.35$ (ethyl acetate: $\left.100 \%\right) ;{ }^{1} \mathrm{H} \mathrm{NMR}\left(400 \mathrm{MHz}, \mathrm{CDCl}_{3}\right) \delta$ $8.45(\mathrm{~s}, 1 \mathrm{H}), 8.15(\mathrm{~d}, J=6.5 \mathrm{~Hz}, 1 \mathrm{H}), 7.67-7.56(\mathrm{~m}, 1 \mathrm{H}), 7.10-7.01(\mathrm{~m}, 1 \mathrm{H}) ;{ }^{13} \mathrm{C}$ NMR $(101$ $\left.\mathrm{MHz}, \mathrm{CDCl}_{3}\right) \delta 150.7,134.5,134.2,116.0$; IR (neat): 3440, 1581, 1531, 1415, 1306, $793 \mathrm{~cm}^{-1}$; HRMS (Q-TOF) $m / z$ calc. for $\mathrm{C}_{4} \mathrm{H}_{4} \mathrm{~N}_{2} \mathrm{O}(\mathrm{M})^{+}$96.0324, found 96.0310 .

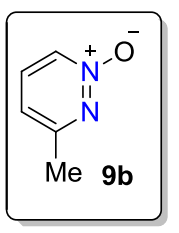

3-Methylpyridazine 1-oxide (9b): Prepared by following General procedure C. Obtained as a colorless liquid $(0.060 \mathrm{~g}$, Yield $=55 \%) ; \mathrm{R}_{\mathrm{f}}=0.3$ (ethyl acetate: $\left.100 \%\right) ;{ }^{1} \mathrm{H} \mathrm{NMR}(500 \mathrm{MHz}$, $\left.\mathrm{CDCl}_{3}\right) \delta 8.04-8.02(\mathrm{~m}, 1 \mathrm{H}), 7.49(\mathrm{dd}, J=7.8,6.4 \mathrm{~Hz}, 1 \mathrm{H}), 6.92(\mathrm{~d}, J=7.9 \mathrm{~Hz}, 1 \mathrm{H}), 2.53(\mathrm{~s}$, $3 \mathrm{H}) ;{ }^{13} \mathrm{C}$ NMR $\left(176 \mathrm{MHz}, \mathrm{CDCl}_{3}\right) \delta 161.0,133.7,131.5,116.7,21.8$; IR (neat): 3450, 1585, 1280, 1150, 960, $780 \mathrm{~cm}^{-1}$; HRMS (Q-TOF) $\mathrm{m} / z$ calc. for $\mathrm{C}_{5} \mathrm{H}_{6} \mathrm{~N}_{2} \mathrm{O}(\mathrm{M})^{+} 110.0474$, found 110.0455 .

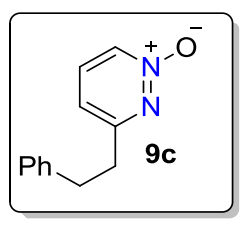

3-Phenethylpyridazine 1-oxide (9c): Prepared by following General procedure C. Obtained as a white solid $(0.100 \mathrm{~g}$, Yield $=50 \%)$; m.p. $=98-100{ }^{\circ} \mathrm{C} ; \mathrm{R}_{\mathrm{f}}=0.3$ (ethyl acetate: $\left.100 \%\right) ;{ }^{1} \mathrm{H}$ NMR $\left(400 \mathrm{MHz}, \mathrm{CDCl}_{3}\right) \delta 8.00(\mathrm{~d}, J=6.3 \mathrm{~Hz}, 1 \mathrm{H}), 7.43(\mathrm{dd}, J=7.8,6.4 \mathrm{~Hz}, 1 \mathrm{H}), 7.27-7.20(\mathrm{~m}, 2 \mathrm{H})$, $7.19-7.11(\mathrm{~m}, 3 \mathrm{H}), 6.75(\mathrm{~d}, J=7.9 \mathrm{~Hz}, 1 \mathrm{H}), 3.03(\mathrm{~s}, 4 \mathrm{H}) ;{ }^{13} \mathrm{C} \mathrm{NMR}\left(101 \mathrm{MHz}, \mathrm{CDCl}_{3}\right) \delta$ 163.5, 140.1, 133.7, 131.8, 128.6, 128.5, 126.4, 116.5, 37.3, 34.9; IR (neat): 3404, 2924, 2863 , 1654, 1573, 1409, 1236, 1016, $798 \mathrm{~cm}^{-1}$; HRMS (ESI) $\mathrm{m} / z$ calc. for $\mathrm{C}_{12} \mathrm{H}_{12} \mathrm{~N}_{2} \mathrm{O}(\mathrm{M}+\mathrm{H})^{+}$ 201.1022, found 201.1044.

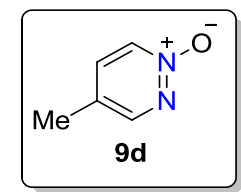

4-Methylpyridazine 1-oxide(9d): Prepared by following General procedure C. Obtained as a colorless liquid $(0.049 \mathrm{~g}$, Yield $=45 \%) ; \mathrm{R}_{\mathrm{f}}=0.3$ (ethyl acetate: $\left.100 \%\right) ;{ }^{1} \mathrm{H}$ NMR $(400 \mathrm{MHz}$, $\left.\mathrm{CDCl}_{3}\right) \delta 8.31(\mathrm{~d}, J=4.6 \mathrm{~Hz}, 1 \mathrm{H}), 8.04(\mathrm{~s}, 1 \mathrm{H}), 6.89(\mathrm{~d}, J=5.5 \mathrm{~Hz}, 1 \mathrm{H}), 2.33(\mathrm{~s}, 3 \mathrm{H}) ;{ }^{13} \mathrm{C} \mathrm{NMR}$ 
$\left(101 \mathrm{MHz}, \mathrm{CDCl}_{3}\right) \delta 149.6,146.3,134.5,117.6,18.3 ; \mathrm{IR}$ (neat): 3410, 2922, 2853, 1620, 1559, 1438, 1382, 1306, 1190, 999, $831 \mathrm{~cm}^{-1}$; HRMS (ESI) $\mathrm{m} / z$ calc. for $\mathrm{C}_{5} \mathrm{H}_{6} \mathrm{~N}_{2} \mathrm{O}(\mathrm{M}+\mathrm{Na})^{+} 133.0372$, found 133.0389 .

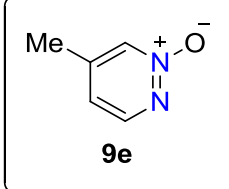

5-Methylpyridazine 1-oxide (9e): Prepared by following General procedure C. Obtained as a colorless liquid $(0.046 \mathrm{~g}$, Yield $=42 \%) ; \mathrm{R}_{\mathrm{f}}=0.3$ (ethyl acetate: $\left.100 \%\right) ;{ }^{1} \mathrm{H} \mathrm{NMR}(500 \mathrm{MHz}$, $\left.\mathrm{CDCl}_{3}\right) \delta 8.23(\mathrm{~s}, 1 \mathrm{H}), 8.06-7.98(\mathrm{~m}, 1 \mathrm{H}), 7.39(\mathrm{~d}, J=3.9 \mathrm{~Hz}, 1 \mathrm{H}), 2.28(\mathrm{~d}, J=4.2 \mathrm{~Hz}, 3 \mathrm{H}) ;{ }^{13} \mathrm{C}$ NMR (126 MHz, $\left.\mathrm{CDCl}_{3}\right) \delta 150.9,134.6,133.4,127.4,17.4$; IR (neat): 3460, 2929, 2853, 1643 , 1550, 1456, 1294, 1020, $846 \mathrm{~cm}^{-1}$; HRMS (ESI) $\mathrm{m} / z$ calc. for $\mathrm{C}_{5} \mathrm{H}_{6} \mathrm{~N}_{2} \mathrm{O}(\mathrm{M}+\mathrm{Na})^{+} 133.0372$, found 133.0384 .

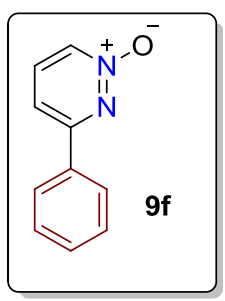

3-Phenylpyridazine 1-oxide (9f): Prepared by following General procedure C. Obtained as a white solid (0.163 g, Yield =95\%); m.p.= $102-104{ }^{\circ} \mathrm{C} ; \mathrm{R}_{\mathrm{f}}=0.35$ (ethyl acetate: $100 \%$ ); ${ }^{1} \mathrm{H}$ NMR (400 MHz, $\left.\mathrm{CDCl}_{3}\right) \delta 8.12-8.09(\mathrm{~m}, 1 \mathrm{H}), 7.98-7.92(\mathrm{~m}, 2 \mathrm{H}), 7.65(\mathrm{dd}, J=8.1,6.3 \mathrm{~Hz}$, 1H), $7.50-7.42(\mathrm{~m}, 4 \mathrm{H}) ;{ }^{13} \mathrm{C} \mathrm{NMR}\left(101 \mathrm{MHz}, \mathrm{CDCl}_{3}\right) \delta 159.7,134.2,133.8,132.3,131.1$, 129.2, 127.2, 113.7; IR (neat): 3460, 1555, 1410, 1334, 1270, 985, 767, $729 \mathrm{~cm}^{-1}$; HRMS (QTOF) $m / z$ calc. for $\mathrm{C}_{10} \mathrm{H}_{8} \mathrm{~N}_{2} \mathrm{O}(\mathrm{M})^{+} 172.0594$, found 172.0581 .

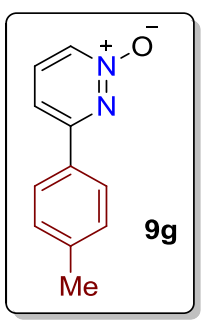

3-(p-Tolyl)pyridazine 1-oxide (9g): Prepared by following General procedure C. Obtained as a colorless liquid $(0.167 \mathrm{~g}$, Yield $=90 \%) ; \mathrm{R}_{\mathrm{f}}=0.35$ (ethyl acetate: $\left.100 \%\right) ;{ }^{1} \mathrm{H}$ NMR $(400 \mathrm{MHz}$, $\left.\mathrm{CDCl}_{3}\right) \delta 8.10(\mathrm{~d}, J=6.2 \mathrm{~Hz}, 1 \mathrm{H}), 7.87(\mathrm{~d}, J=8.2 \mathrm{~Hz}, 2 \mathrm{H}), 7.62(\mathrm{dd}, J=8.1,6.3 \mathrm{~Hz}, 1 \mathrm{H}), 7.43$ 
$(\mathrm{d}, J=8.1 \mathrm{~Hz}, 1 \mathrm{H}), 7.28(\mathrm{~d}, J=8.0 \mathrm{~Hz}, 2 \mathrm{H}), 2.40(\mathrm{~s}, 3 \mathrm{H}) ;{ }^{13} \mathrm{C} \mathrm{NMR}\left(101 \mathrm{MHz}, \mathrm{CDCl}_{3}\right) \delta$ 159.7, 141.6, 134.1, 131.9, 131.1, 129.9, 127.2, 113.4, 21.5; IR (neat): 3405, 3060, 2922, 2853, 1620, 1550, 1205, $760 \mathrm{~cm}^{-1}$; HRMS (ESI) $\mathrm{m} / z$ calc. for $\mathrm{C}_{11} \mathrm{H}_{10} \mathrm{~N}_{2} \mathrm{O}(\mathrm{M}+\mathrm{Na})^{+} 187.0866$, found 187.0893 .

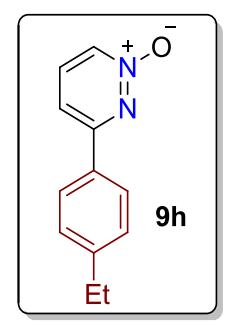

3-(4-Ethylphenyl)pyridazine 1-oxide (9h): Prepared by following General procedure C. Obtained as a white solid $(0.192 \mathrm{~g}$, Yield $=96 \%) ; \mathrm{m} . \mathrm{p} .=142-144{ }^{\circ} \mathrm{C} ; \mathrm{R}_{\mathrm{f}}=0.35$ (ethyl acetate: 100\%); ${ }^{1} \mathrm{H}$ NMR (400 MHz, $\left.\mathrm{CDCl}_{3}\right) \delta 8.08(\mathrm{~d}, J=6.2 \mathrm{~Hz}, 1 \mathrm{H}), 7.88(\mathrm{~d}, J=8.3 \mathrm{~Hz}, 2 \mathrm{H}), 7.62$ (dd, $J=8.2,6.2 \mathrm{~Hz}, 1 \mathrm{H}), 7.45-7.40(\mathrm{~m}, 1 \mathrm{H}), 7.30(\mathrm{~d}, J=8.2 \mathrm{~Hz}, 2 \mathrm{H}), 2.69$ (q, $J=7.6 \mathrm{~Hz}, 2 \mathrm{H})$, $1.25(\mathrm{t}, J=7.6 \mathrm{~Hz}, 3 \mathrm{H}) ;{ }^{13} \mathrm{C}$ NMR $\left(101 \mathrm{MHz}, \mathrm{CDCl}_{3}\right) \delta 159.7,147.8,134.1,131.9,131.2,128.7$, 127.2, 113.4, 28.8, 15.3; IR (neat): 3431, 2963, 2853, 1580, 1440, 1380, 1271,767 $\mathrm{cm}^{-1}$; HRMS (ESI) $m / z$ calc. for $\mathrm{C}_{12} \mathrm{H}_{12} \mathrm{~N}_{2} \mathrm{O}(\mathrm{M}+\mathrm{Na})^{+} 223.0842$, found 223.0838 .

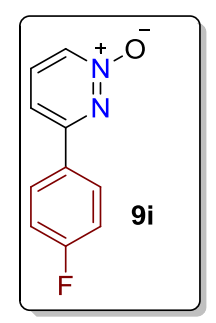

3-(4-Fluorophenyl)pyridazine 1-oxide (9i): Prepared by following General procedure C. Obtained as a white solid $(0.178 \mathrm{~g}$, Yield $=94 \%)$; m.p. $=134-136{ }^{\circ} \mathrm{C} ; \mathrm{R}_{\mathrm{f}}=0.35$ (ethyl acetate: 100\%); ${ }^{1} \mathrm{H}$ NMR $\left(400 \mathrm{MHz}, \mathrm{CDCl}_{3}\right) \delta 8.11-8.07(\mathrm{~m}, 1 \mathrm{H}), 7.97-7.91(\mathrm{~m}, 2 \mathrm{H}), 7.64(\mathrm{dd}, J=$ 8.1, $6.3 \mathrm{~Hz}, 1 \mathrm{H}), 7.42-7.38(\mathrm{~m}, 1 \mathrm{H}), 7.17-7.10(\mathrm{~m}, 2 \mathrm{H}) ;{ }^{13} \mathrm{C} \mathrm{NMR}\left(101 \mathrm{MHz}, \mathrm{CDCl}_{3}\right) \delta 164.6$ $(\mathrm{d}, J=252.1 \mathrm{~Hz}), 158.6,134.3,132.2,130.0(\mathrm{~d}, J=3.2 \mathrm{~Hz}), 129.3(\mathrm{~d}, J=8.8 \mathrm{~Hz}), 116.3(\mathrm{~d}, J=$ $21.9 \mathrm{~Hz}), 113.3$; IR (neat): 3442, 1575, 1458, 1380, 1271,1100, 980, $730 \mathrm{~cm}^{-1}$; HRMS (ESI) $\mathrm{m} / 2$ calc. for $\mathrm{C}_{10} \mathrm{H}_{7} \mathrm{FN}_{2} \mathrm{O}(\mathrm{M}+\mathrm{Na})^{+} 213.0435$, found 213.0447. 


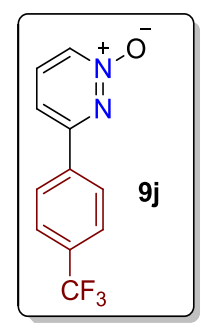

3-(4-(Trifluoromethyl)phenyl)pyridazine 1-oxide (9j): Prepared by following General procedure C. Obtained as a white solid $(0.235 \mathrm{~g}$, Yield $=98 \%)$; m.p. $=156-158{ }^{\circ} \mathrm{C} ; \mathrm{R}_{\mathrm{f}}=0.35$ (ethyl acetate: $100 \%) ;{ }^{1} \mathrm{H}$ NMR $\left(400 \mathrm{MHz}, \mathrm{CDCl}_{3}\right) \delta 8.17(\mathrm{~d}, J=6.3 \mathrm{~Hz}, 1 \mathrm{H}), 8.09(\mathrm{~d}, J=8.2$ $\mathrm{Hz}, 2 \mathrm{H}), 7.77-7.68(\mathrm{~m}, 3 \mathrm{H}), 7.49(\mathrm{~d}, J=8.1 \mathrm{~Hz}, 1 \mathrm{H}) ;{ }^{13} \mathrm{C} \mathrm{NMR}\left(101 \mathrm{MHz}, \mathrm{CDCl}_{3}\right) \delta 158.3$, 137.2, 134.5, 133.2, $132.9(\mathrm{~d}, J=32.8 \mathrm{~Hz}), 127.7,126.2(\mathrm{q}, J=3.8 \mathrm{~Hz}), 123.8(\mathrm{q}, J=272.5 \mathrm{~Hz})$, 113.8; IR (neat): 3433, 1615, 1565, 1332, 961, $765 \mathrm{~cm}^{-1}$; HRMS (ESI) $m / z$ calc. for $\mathrm{C}_{11} \mathrm{H}_{7} \mathrm{~F}_{3} \mathrm{~N}_{2} \mathrm{O}$ $(\mathrm{M}+\mathrm{Na})^{+}$263.0403, found 263.0386 .

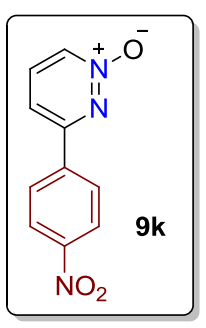

3-(4-Nitrophenyl)pyridazine 1-oxide (9k): Prepared by following General procedure C. Obtained as a pale yellow solid $(0.206 \mathrm{~g}$, Yield $=95 \%) ;$ m.p. $=210-212{ }^{\circ} \mathrm{C} ; \mathrm{R}_{\mathrm{f}}=0.35$ (ethyl acetate: $100 \%) ;{ }^{1} \mathrm{H}$ NMR $\left(500 \mathrm{MHz}, \mathrm{CDCl}_{3}\right) \delta 8.38-8.33(\mathrm{~m}, 2 \mathrm{H}), 8.21-8.19(\mathrm{~m}, 1 \mathrm{H}), 8.18-$ $8.14(\mathrm{~m}, 2 \mathrm{H}), 7.75(\mathrm{dd}, J=8.0,6.4 \mathrm{~Hz}, 1 \mathrm{H}), 7.52(\mathrm{~d}, J=8.1 \mathrm{~Hz}, 1 \mathrm{H}) ;{ }^{13} \mathrm{C} \mathrm{NMR}(126 \mathrm{MHz}$, $\left.\mathrm{CDCl}_{3}\right) \delta 157.4,149.5,139.7,134.6,133.7,128.3,124.4,113.9$; IR (neat): 3437, 3091, 1645, 1512, 1415, 1344, 1018, $850 \mathrm{~cm}^{-1}$; HRMS (ESI) $\mathrm{m} / z$ calc. for $\mathrm{C}_{10} \mathrm{H}_{7} \mathrm{~N}_{3} \mathrm{O}_{3}(\mathrm{M}+\mathrm{H})^{+} 218.0560$, found 218.0588 .

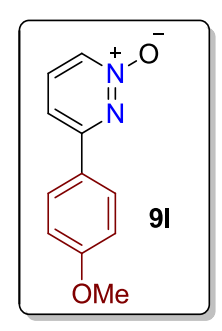

3-(4-Methoxyphenyl)pyridazine 1-oxide (91): Prepared by following General procedure C. Obtained as a white solid m.p. $=134-136{ }^{\circ} \mathrm{C} ; \mathrm{R}_{\mathrm{f}}=0.35$ (ethyl acetate: $\left.100 \%\right) ;{ }^{1} \mathrm{H}$ NMR $(400$ $\left.\mathrm{MHz}, \mathrm{CDCl}_{3}\right) \delta 8.08(\mathrm{~d}, J=6.2 \mathrm{~Hz}, 1 \mathrm{H}), 7.97-7.93(\mathrm{~m}, 2 \mathrm{H}), 7.60(\mathrm{dd}, J=8.2,6.2 \mathrm{~Hz}, 1 \mathrm{H})$, 
$7.40(\mathrm{~d}, J=8.2 \mathrm{~Hz}, 1 \mathrm{H}), 7.03-6.97(\mathrm{~m}, 2 \mathrm{H}), 3.87(\mathrm{~s}, 3 \mathrm{H}) ;{ }^{13} \mathrm{C} \mathrm{NMR}\left(101 \mathrm{MHz}, \mathrm{CDCl}_{3}\right) \delta$ 162.2, 159.4, 134.0, 131.6, 128.8, 126.2, 114.6, 112.9, 55.6; IR (neat): 3450, 2956, 2823, 1635, 1580, 1332, 1230, 1165, $961 \mathrm{~cm}^{-1}$; HRMS (ESI) $\mathrm{m} / z$ calc. for $\mathrm{C}_{11} \mathrm{H}_{10} \mathrm{~N}_{2} \mathrm{O}_{2}(\mathrm{M}+\mathrm{Na})^{+} 225.0634$, found 225.0648 .

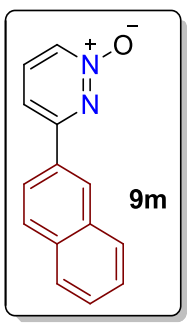

3-(Naphthalen-2-yl)pyridazine 1-oxide (9m): Prepared by following General procedure C. Obtained as a white solid $(0.208 \mathrm{~g}$, Yield $=94 \%) ; \mathrm{m} . \mathrm{p} .=196-198{ }^{\circ} \mathrm{C} ; \mathrm{R}_{\mathrm{f}}=0.4$ (ethyl acetate: 100\%); ${ }^{1} \mathrm{H}$ NMR (400 MHz, $\left.\mathrm{CDCl}_{3}\right) \delta 8.52(\mathrm{~d}, \mathrm{~J}=1.3 \mathrm{~Hz}, 1 \mathrm{H}), 8.14(\mathrm{dd}, \mathrm{J}=6.2,0.5 \mathrm{~Hz}, 1 \mathrm{H})$, $8.04(\mathrm{dd}, J=8.6,1.9 \mathrm{~Hz}, 1 \mathrm{H}), 7.97-7.91(\mathrm{~m}, 2 \mathrm{H}), 7.88(\mathrm{dd}, J=7.7,5.3 \mathrm{~Hz}, 1 \mathrm{H}), 7.67$ (dd, $J=$ 8.1, $6.2 \mathrm{~Hz}, 1 \mathrm{H}), 7.61-7.51(\mathrm{~m}, 3 \mathrm{H}) ;{ }^{13} \mathrm{C} \mathrm{NMR}\left(101 \mathrm{MHz}, \mathrm{CDCl}_{3}\right) \delta 159.6,134.6,134.2,133.3$, 132.3, 131.0, 129.1, 129.1, 127.9, 127.8, 127.7, 127.1, 123.8, 113.8; IR (neat): 3450, 2749, $1715,1664,1543,1250,1113,885,730 \mathrm{~cm}^{-1} \mathrm{HRMS}(\mathrm{ESI}) \mathrm{m} / \mathrm{z}$ calc. for $\mathrm{C}_{14} \mathrm{H}_{10} \mathrm{~N}_{2} \mathrm{O}(\mathrm{M}+\mathrm{Na})^{+}$ 245.0685 , found 245.0701 .

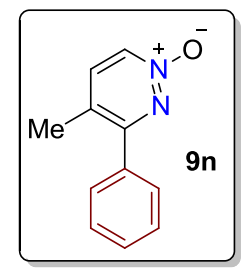

4-Methyl-3-phenylpyridazine 1-oxide (9n): Prepared by following General procedure C. Obtained as a white solid $(0.180 \mathrm{~g}$, Yield $=97 \%) ; \mathrm{m} . \mathrm{p} .=96-98{ }^{\circ} \mathrm{C} ; \mathrm{R}_{\mathrm{f}}=0.4$ (ethyl acetate: 100\%); ${ }^{1} \mathrm{H}$ NMR (400 MHz, $\left.\mathrm{CDCl}_{3}\right) \delta 8.04(\mathrm{~d}, J=6.4 \mathrm{~Hz}, 1 \mathrm{H}), 7.51-7.44(\mathrm{~m}, 3 \mathrm{H}), 7.44-7.39$ (m, 3H), $2.28(\mathrm{~s}, 3 \mathrm{H}) ;{ }^{13} \mathrm{C}$ NMR $\left(101 \mathrm{MHz}, \mathrm{CDCl}_{3}\right) \delta 160.7,135.9,134.8,132.0,129.6,129.0$, 128.4, 126.1, 18.6; IR (neat): 3420, 2963, 2925, 1530, 1280, 994, 871, $768 \mathrm{~cm}^{-1}$; HRMS (ESI) $\mathrm{m} / \mathrm{z}$ calc. for $\mathrm{C}_{11} \mathrm{H}_{10} \mathrm{~N}_{2} \mathrm{O}(\mathrm{M}+\mathrm{H})^{+}$187.0866, found 187.0894 . 


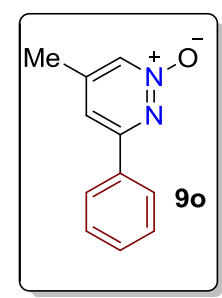

5-Methyl-3-phenylpyridazine 1-oxide (9o): Prepared by following General procedure C. Obtained as a semisolid (0.176 $\mathrm{g}$, Yield $=95 \%) ; \mathrm{R}_{\mathrm{f}}=0.4$ (ethyl acetate:100); ${ }^{1} \mathrm{H}$ NMR (400 $\left.\mathrm{MHz}, \mathrm{CDCl}_{3}\right) \delta 8.00(\mathrm{~s}, 1 \mathrm{H}), 7.94(\mathrm{dd}, J=6.5,3.1 \mathrm{~Hz}, 2 \mathrm{H}), 7.49-7.44(\mathrm{~m}, 3 \mathrm{H}), 7.28(\mathrm{~s}, 1 \mathrm{H})$, 2.37 (s, 3H); ${ }^{13} \mathrm{C}$ NMR (101 MHz, $\left.\mathrm{CDCl}_{3}\right) \delta$ 158.7, 146.2, 134.0, 132.5, 130.9, 129.1, 127.3, 115.3, 18.5; IR (neat): 3350, 2749, 1700, 1620, 1543, 1250, 1080, 885, 735; HRMS (ESI) m/z calc. for $\mathrm{C}_{11} \mathrm{H}_{10} \mathrm{~N}_{2} \mathrm{O}(\mathrm{M}+\mathrm{H})^{+}$187.0866, found 187.0894.

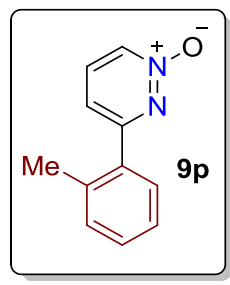

3-(o-Tolyl)pyridazine 1-oxide (9p): Prepared by following General procedure C. Obtained as a colorless semisolid $(0.178 \mathrm{~g}$, Yield $=96 \%) ; \mathrm{R}_{\mathrm{f}}=0.35$ (ethyl acetate: $\left.100 \%\right) ;{ }^{1} \mathrm{H}$ NMR $(400$ $\left.\mathrm{MHz} \mathrm{CDCl}_{3}\right) \delta 8.15(\mathrm{~d}, J=6.3 \mathrm{~Hz}, 1 \mathrm{H}), 7.65(\mathrm{dd}, J=7.8,6.5 \mathrm{~Hz}, 1 \mathrm{H}), 7.42-7.34(\mathrm{~m}, 2 \mathrm{H}), 7.32$ - $7.26(\mathrm{~m}, 2 \mathrm{H}), 7.14(\mathrm{~d}, J=8.0 \mathrm{~Hz}, 1 \mathrm{H}), 2.41(\mathrm{~s}, 3 \mathrm{H}) ;{ }^{13} \mathrm{C} \mathrm{NMR}\left(101 \mathrm{MHz}, \mathrm{CDCl}_{3}\right) \delta 162.4$, 136.3, 134.9, 133.7, 132.1, 131.3, 130.0, 129.7, 126.3, 117.2, 20.5; IR (neat): 3451, 2916, 1738, 1540, 1297, 1063, 994, $768 \mathrm{~cm}^{-1}$; HRMS (ESI) $\mathrm{m} /$ calc. for $\mathrm{C}_{11} \mathrm{H}_{10} \mathrm{~N}_{2} \mathrm{O}(\mathrm{M}+\mathrm{H})^{+} 187.0866$, found 187.0888 .

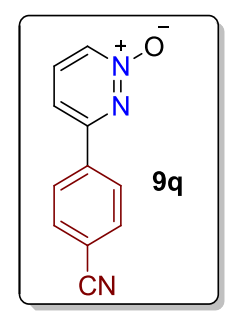

3-(4-Cyanophenyl)pyridazine 1-oxide (9q): Prepared by following General procedure C. Obtained as a pale yellow solid $(0.177 \mathrm{~g}$, Yield $=90 \%) ; \mathrm{m} . \mathrm{p} .=218-220{ }^{0} \mathrm{C} ; \mathrm{R}_{\mathrm{f}}=0.35$ (ethyl acetate: $100 \%) ;{ }^{1} \mathrm{H} \mathrm{NMR}\left(500 \mathrm{MHz}, \mathrm{CDCl}_{3}\right) \delta 8.18(\mathrm{~d}, J=6.3 \mathrm{~Hz}, 1 \mathrm{H}), 8.12-8.07(\mathrm{~m}, 2 \mathrm{H})$, $7.83-7.78(\mathrm{~m}, 2 \mathrm{H}), 7.73(\mathrm{dd}, J=8.1,6.4 \mathrm{~Hz}, 1 \mathrm{H}), 7.50-7.46(\mathrm{~m}, 1 \mathrm{H}) ;{ }^{13} \mathrm{C} \mathrm{NMR}(126 \mathrm{MHz}$, 
$\left.\mathrm{CDCl}_{3}\right) \delta 157.7,137.9,134.6,133.5,133.0,127.9,118.2,114.8,113.7$; IR (neat): 3433, 3050, $1583,1423,1267,1166,981,750 \mathrm{~cm}^{-1}$; HRMS (ESI) $\mathrm{m} / z$ calc. for $\mathrm{C}_{11} \mathrm{H}_{7} \mathrm{~N}_{3} \mathrm{O}(\mathrm{M}+\mathrm{H})^{+} 198.0662$, found 198.0660 .

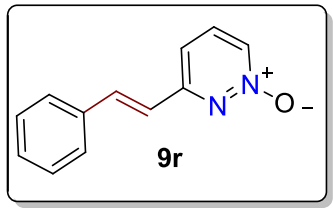

(E)-3-Styrylpyridazine 1-oxide (9r): Prepared by following General procedure C. Obtained as a brown solid $(0.122 \mathrm{~g}$, Yield $=62 \%) ; \mathrm{m} . \mathrm{p} .=206-208{ }^{\circ} \mathrm{C} ; \mathrm{R}_{\mathrm{f}}=0.5$ (ethyl acetate:100); ${ }^{1} \mathrm{H}$ NMR $\left(400 \mathrm{MHz}, \mathrm{CDCl}_{3}\right) \delta 8.04(\mathrm{~d}, J=6.2 \mathrm{~Hz}, 1 \mathrm{H}), 7.75(\mathrm{~d}, J=16.2 \mathrm{~Hz}, 1 \mathrm{H}), 7.58-7.52(\mathrm{~m}, 3 \mathrm{H})$, $7.43-7.31(\mathrm{~m}, 3 \mathrm{H}), 7.11(\mathrm{~d}, J=8.0 \mathrm{~Hz}, 1 \mathrm{H}), 6.95(\mathrm{~d}, J=16.2 \mathrm{~Hz}, 1 \mathrm{H}) ;{ }^{13} \mathrm{C}$ NMR $(101 \mathrm{MHz}$, $\left.\mathrm{CDCl}_{3}\right) \delta 158.0,137.5,135.4,133.9,132.2,129.7,129.1,127.7,122.1,114.8$; IR (neat): 3441, 3061, 1636, 1562, 1330, 1153, 979, $798 \mathrm{~cm}^{-1}$; HRMS (ESI) m/zcalc. for $\mathrm{C}_{12} \mathrm{H}_{10} \mathrm{~N}_{2} \mathrm{O}(\mathrm{M}+\mathrm{H})^{+}$ 199.0866, found 199.0889 .

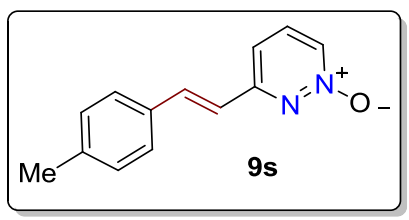

(E)-3-(4-Methylstyryl)pyridazine 1-oxide (9s): Prepared by following General procedure C. Obtained as a yellow solid $(0.137 \mathrm{~g}$, Yield $=65 \%)$; m.p. $=158-160{ }^{0} \mathrm{C} ; \mathrm{R}_{\mathrm{f}}=0.4$ (ethyl acetate:100); ${ }^{1} \mathrm{H}$ NMR (400 MHz, $\left.\mathrm{CDCl}_{3}\right) \delta 8.00(\mathrm{~d}, J=6.2 \mathrm{~Hz}, 1 \mathrm{H}), 7.69(\mathrm{~d}, J=16.2 \mathrm{~Hz}, 1 \mathrm{H})$, $7.52(\mathrm{dd}, J=7.9,6.3 \mathrm{~Hz}, 1 \mathrm{H}), 7.42(\mathrm{~d}, J=8.0 \mathrm{~Hz}, 2 \mathrm{H}), 7.17(\mathrm{~d}, J=7.9 \mathrm{~Hz}, 2 \mathrm{H}), 7.08$ (d, $J=8.0$ $\mathrm{Hz}, 1 \mathrm{H}), 6.89(\mathrm{~d}, J=16.2 \mathrm{~Hz}, 1 \mathrm{H}), 2.35(\mathrm{~s}, 3 \mathrm{H}) ;{ }^{13} \mathrm{C} \mathrm{NMR}\left(101 \mathrm{MHz}, \mathrm{CDCl}_{3}\right) \delta 158.2,140.0$, 137.4, 133.8, 132.6, 131.9, 129.7, 127.6, 121.1, 114.7, 21.5; IR (neat): 3446, 3095, 2956, 2920, 1637, 1568, 1417, 1161, 810, $750 \mathrm{~cm}^{-1}$; HRMS (ESI) m/z calc. for $\mathrm{C}_{13} \mathrm{H}_{12} \mathrm{~N}_{2} \mathrm{O}(\mathrm{M}+\mathrm{H})^{+} 213.1022$, found 213.1048 .

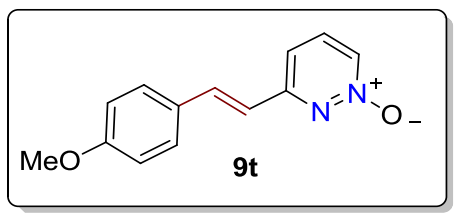

(E)-3-(4-Methoxystyryl)pyridazine 1-oxide (9t): Prepared by following General procedure $\mathbf{C}$. Obtained as a brown solid $(0.136 \mathrm{~g}$, Yield $=60 \%) ; \mathrm{m} . \mathrm{p}=130-132{ }^{0} \mathrm{C} ; \mathrm{R}_{\mathrm{f}}=0.4$ (ethyl 
acetate:100); ${ }^{1} \mathrm{H} \mathrm{NMR}\left(400 \mathrm{MHz}, \mathrm{CDCl}_{3}\right) \delta 7.98(\mathrm{~d}, J=6.2 \mathrm{~Hz}, 1 \mathrm{H}), 7.67(\mathrm{~d}, J=16.1 \mathrm{~Hz}, 1 \mathrm{H})$, $7.53-7.41(\mathrm{~m}, 3 \mathrm{H}), 7.06(\mathrm{~d}, J=8.1 \mathrm{~Hz}, 1 \mathrm{H}), 6.89(\mathrm{~d}, J=8.6 \mathrm{~Hz}, 2 \mathrm{H}), 6.79(\mathrm{~d}, J=16.1 \mathrm{~Hz}, 1 \mathrm{H})$, $3.81(\mathrm{~s}, 3 \mathrm{H}) ;{ }^{13} \mathrm{C} \mathrm{NMR}\left(101 \mathrm{MHz}, \mathrm{CDCl}_{3}\right) \delta 160.9,158.3,137.0,133.8,131.7,129.2,128.1$, 119.7, 114.6, 114.5, 55.4; IR (neat): 3448, 3064, 2935, 2837, 1636, 1566, 1421, 1174, 1026, 826 $\mathrm{cm}^{-1}$; HRMS (ESI) m/z calc. for $\mathrm{C}_{13} \mathrm{H}_{12} \mathrm{~N}_{2} \mathrm{O}_{2}(\mathrm{M}+\mathrm{H})^{+} 229.0972$, found 229.0998.

\section{c) Preparation of pyridazinium salts 6}

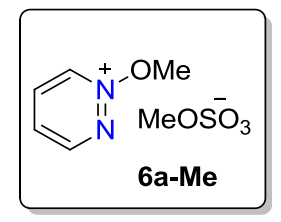

1-Methoxypyridazin-1-ium methyl sulfate (6a-Me): A mixture of pyridazine 1-oxide 9a (1 mmol, $96 \mathrm{mg})$ and dimethyl sulfate $(1.2 \mathrm{mmol}, 151 \mathrm{mg})$ was stirred at $70{ }^{\circ} \mathrm{C}$ for $2 \mathrm{~h}$. The reaction mixture was cooled to rt and washed with diethyl ether and dried under vacuum to obtain the salt 6a-Me as a brown liquid $(0.170 \mathrm{~g}$, Yield $=78 \%) ; \mathrm{R}_{\mathrm{f}}=0.3$ (methanol/DCM : 10:90); ${ }^{1} \mathrm{H}$ NMR $\left(500 \mathrm{MHz}, \mathrm{D}_{2} \mathrm{O}\right) \delta 9.58(\mathrm{~d}, J=6.3 \mathrm{~Hz}, 1 \mathrm{H}), 9.37(\mathrm{~d}, J=3.2 \mathrm{~Hz}, 1 \mathrm{H}), 8.70-8.64(\mathrm{~m}, 1 \mathrm{H}), 8.51-$ $8.44(\mathrm{~m}, 2 \mathrm{H}), 4.48$ (s, 3H), 3.69 (s, 3H); ${ }^{13} \mathrm{C}$ NMR (126 MHz, DMSO- $\left.d_{6}\right) \delta 153.3,140.4,139.0$, 135.5, 64.1, 53.0; IR (neat): 3459, 1644, 1584, 1417, 1221, 1050, $773 \mathrm{~cm}^{-1}$; HRMS (ESI) m/z calc. for $\mathrm{C}_{5} \mathrm{H}_{7} \mathrm{~N}_{2} \mathrm{O}^{+}(\mathrm{M})^{+} 111.0553$, found 111.0570 .

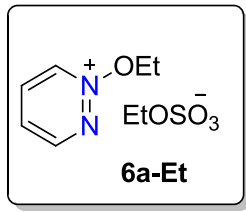

1-Ethoxypyridazin-1-ium ethyl sulfate (6a-Et): A mixture of pyridazine 1-oxide 9a (1 mmol, $96 \mathrm{mg})$ and diethyl sulfate $(1.2 \mathrm{mmol}, 184 \mathrm{mg})$ was stirred at $70{ }^{\circ} \mathrm{C}$ for $2 \mathrm{~h}$. The reaction mixture was cooled to $\mathrm{rt}$ and washed with diethyl ether and dried under vacuum to obtain the salt $\mathbf{6 a - E t}$ as a brown liquid $(0.187 \mathrm{~g}$, Yield $=75 \%) ; \mathrm{R}_{\mathrm{f}}=0.3$ (methanol/DCM : 10:90); ${ }^{1} \mathrm{H}$ NMR $(500$ $\left.\mathrm{MHz}, \mathrm{D}_{2} \mathrm{O}\right) \delta 9.57(\mathrm{~d}, J=6.3 \mathrm{~Hz}, 1 \mathrm{H}), 9.36(\mathrm{dd}, J=4.9,1.8 \mathrm{~Hz}, 1 \mathrm{H}), 8.70-8.64(\mathrm{~m}, 1 \mathrm{H}), 8.52-$ $8.44(\mathrm{~m}, 1 \mathrm{H}), 4.91(\mathrm{q}, J=7.1 \mathrm{~Hz}, 2 \mathrm{H}), 4.06(\mathrm{q}, J=7.1 \mathrm{~Hz}, 2 \mathrm{H}), 1.46(\mathrm{t}, J=7.1 \mathrm{~Hz}, 3 \mathrm{H}), 1.26(\mathrm{t}$, $J=7.1 \mathrm{~Hz}, 3 \mathrm{H}) ;{ }^{13} \mathrm{C}$ NMR $\left(101 \mathrm{MHz}, \mathrm{DMSO}-d_{6}\right) \delta 153.7,141.6,139.2,135.9,75.2,61.4,15.2$, 
12.6; IR (neat): $3454,2952,2854,1652,1583,1430,1330,1228,726 \mathrm{~cm}^{-1}$; HRMS (ESI) $m / z$ calc. for $\mathrm{C}_{6} \mathrm{H}_{9} \mathrm{~N}_{2} \mathrm{O}^{+}(\mathrm{M})^{+} 125.0709$, found 125.0707.

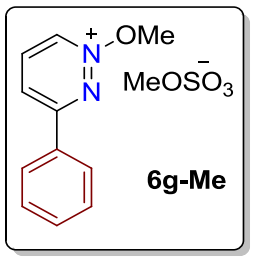

1-Methoxy-3-phenylpyridazin-1-ium methyl sulfate (6g-Me): A mixture of 3phenylpyridazine 1-oxide (1 mmol, $172 \mathrm{mg})$ and dimethyl sulfate $(1.2 \mathrm{mmol}, 151 \mathrm{mg})$ was stirred at $70{ }^{\circ} \mathrm{C}$ for $2 \mathrm{~h}$. The reaction mixture was cooled to $\mathrm{rt}$ and washed with diethyl ether and dried under vacuum to obtain the salt $\mathbf{6 g}$-Me as a brown liquid $(0.290 \mathrm{~g}$, Yield $=98 \%) ; \mathrm{R}_{\mathrm{f}}=0.3$ (methanol/DCM : 10:90); ${ }^{1} \mathrm{H}$ NMR (500 MHz, DMSO- $\left.d_{6}\right) \delta 9.92(\mathrm{~d}, J=6.0 \mathrm{~Hz}, 1 \mathrm{H}), 9.16(\mathrm{~d}, J$ $=8.4 \mathrm{~Hz}, 1 \mathrm{H}), 8.87(\mathrm{dd}, J=8.0,6.5 \mathrm{~Hz}, 1 \mathrm{H}), 8.29(\mathrm{~d}, J=7.3 \mathrm{~Hz}, 2 \mathrm{H}), 7.79-7.62(\mathrm{~m}, 3 \mathrm{H}), 4.58$ (s, 3H), 3.37 (s, 3H); ${ }^{13} \mathrm{C}$ NMR (126 MHz, DMSO- $\left.d_{6}\right) \delta 160.2,139.0,137.8,133.0,132.0,131.4$, 129.7, 128.0, 63.7, 52.8; IR (neat): 3065, 2965, 2925, 1580, 834, $734 \mathrm{~cm}^{-1}$; HRMS (ESI) $\mathrm{m} / \mathrm{z}$ calc. for $\mathrm{C}_{11} \mathrm{H}_{11} \mathrm{~N}_{2} \mathrm{O}^{+}(\mathrm{M})^{+} 187.0866$, found 187.0879.

\section{d) Preparation of substituted pyrroles sa-i}<smiles>c1cc[nH]c1</smiles>

sa<smiles>Cn1cccc1</smiles>

sb<smiles>Cc1ccc[nH]1</smiles>

sc<smiles>Cc1cc[nH]c1</smiles>

sd

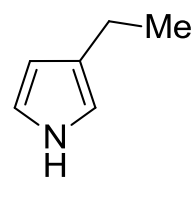

se

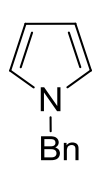

sf<smiles>O=C(Oc1ccccc1)n1cccc1</smiles>

sg

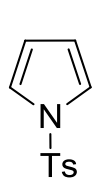

sh<smiles>CC(=O)c1cc[nH]c1</smiles>

si

Pyrroles sa and sb were purchased from sigma aldrich. Pyrroles sc-si were prepared according to reported procedures. ${ }^{8 \mathrm{a}, \mathrm{b}}$

\section{Optimization of transannulation reaction}

Characterization of enaldiazomethane ((E)-4-diazobut-2-enal) 7a:

NOTE: The enaldiazomethane $7 \boldsymbol{a}$ is explosive at room temperature. It should be stored and handled at low temperatures (below $10^{\circ} \mathrm{C}$ ). 


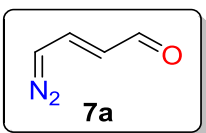

(E)-4-Diazobut-2-enal 7a: ${ }^{9}$ A suspension of 1-methoxypyridazin-1-ium methyl sulfate 6a-Me (222 mg, $2 \mathrm{mmol})$ in DCM $(10 \mathrm{ml})$ at $0{ }^{\circ} \mathrm{C}$ was treated with an ice cold $1 \mathrm{~N}$ aq KOH $(2 \mathrm{ml})$ for 5 minutes. The contents were extracted and the organic phase was dried over anh $\mathrm{Na}_{2} \mathrm{SO}_{4}$ and evaporated under reduced pressure at $0{ }^{\circ} \mathrm{C}$. The enaldiazomethane 7a was purified by a silica gel column chromatography using ethyl acetate and hexane as the eluent. Obtained as an orange red oil $(0.124 \mathrm{~g}$, Yield $=65 \%) ; \mathrm{R}_{\mathrm{f}}=0.3$ (ethyl acetate/hexane : 20:80); ${ }^{1} \mathrm{H}$ NMR $\left(500 \mathrm{MHz}, \mathrm{CDCl}_{3}\right)$ $\delta 9.38(\mathrm{~d}, J=7.8 \mathrm{~Hz}, 1 \mathrm{H}), 7.01(\mathrm{dd}, J=14.6,9.0 \mathrm{~Hz}, 1 \mathrm{H}), 5.94(\mathrm{dd}, J=15.1,7.8 \mathrm{~Hz}, 1 \mathrm{H}), 5.02$ $(\mathrm{d}, J=8.8 \mathrm{~Hz}, 1 \mathrm{H}) ;{ }^{13} \mathrm{C} \mathrm{NMR}\left(176 \mathrm{MHz}, \mathrm{CDCl}_{3}\right) \delta 190.3,144.5,120.8,51.8$ (diazo carbon); IR (neat): 2866, 2762, 2077, 1660, 1571, 1359, 1151, 956, $648 \mathrm{~cm}^{-1}$; HRMS (ESI) $\mathrm{m} / z$ calc. for $\mathrm{C}_{4} \mathrm{H}_{4} \mathrm{~N}_{2} \mathrm{O}(\mathrm{M}+\mathrm{Na})^{+} 119.0216$, found 119.0225 .

\section{Optimization of the additive}

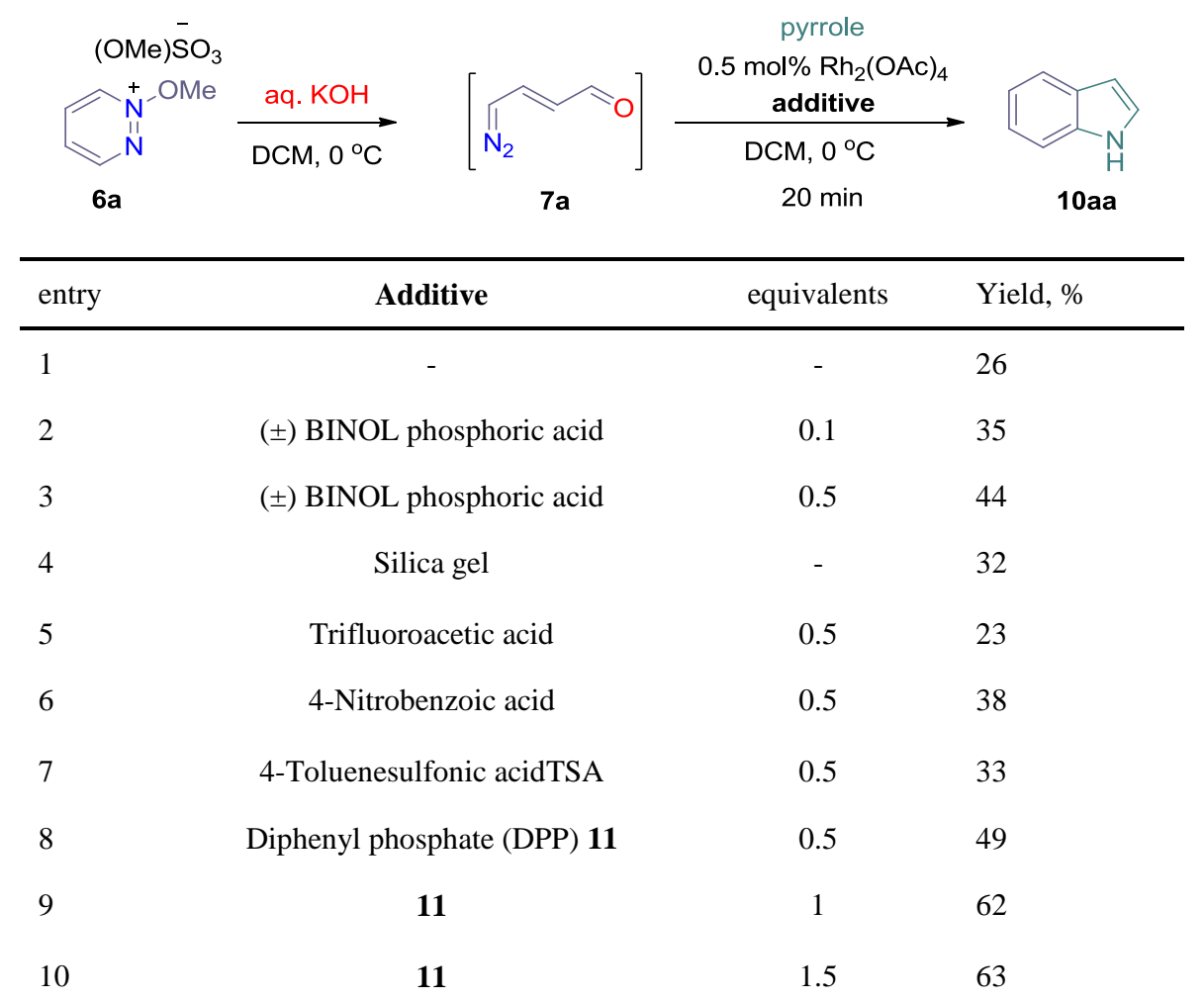

Additive optimization reactions were performed with $0.5 \mathrm{mmol}$ of pyrrole and $1 \mathrm{mmol}$ of salt $6 \mathbf{a}$. 


\section{Optimization of the catalyst and solvent ${ }^{\mathrm{a}}$}

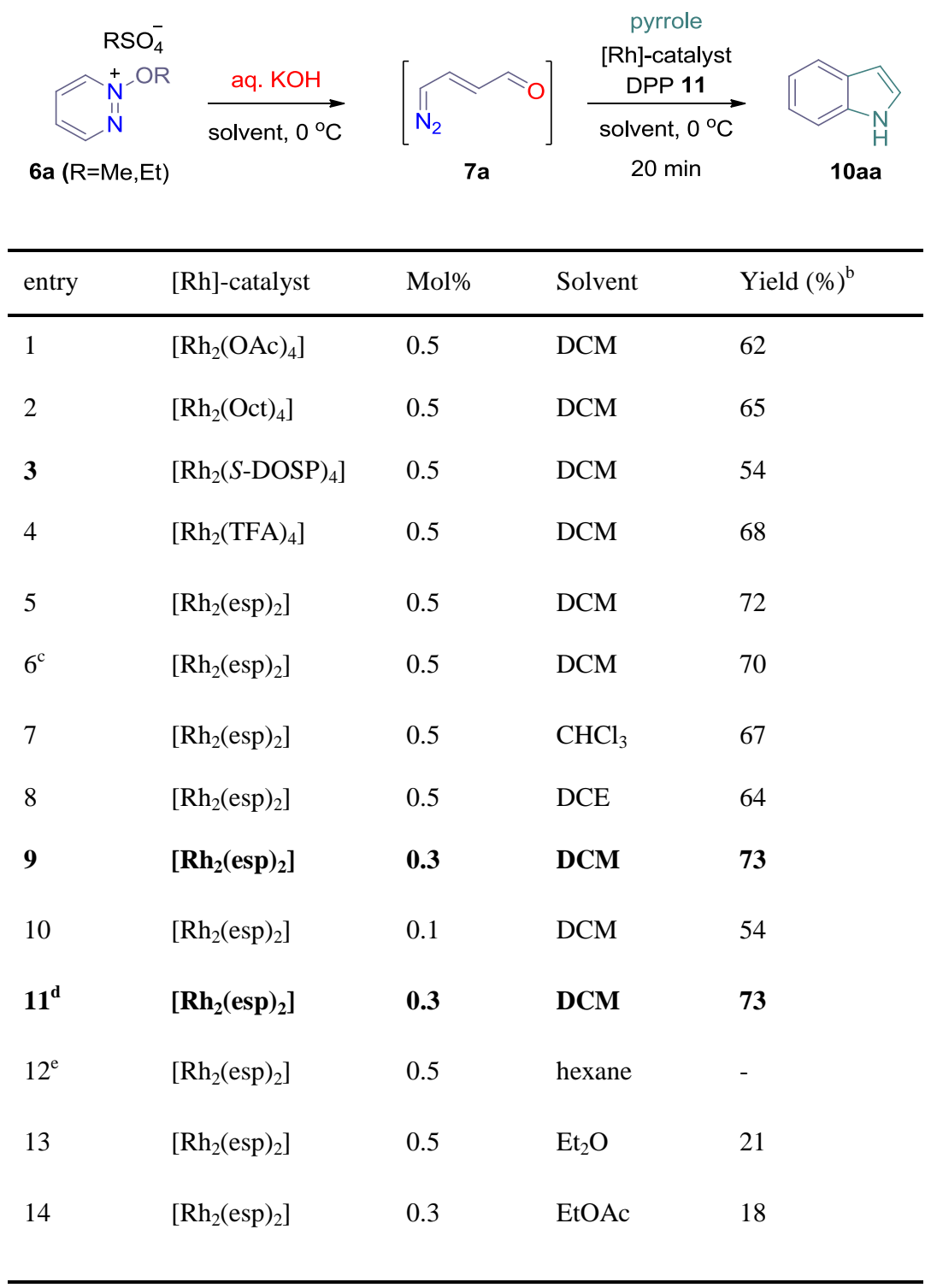

${ }^{\mathrm{a}}$ Reaction conditions: $\mathbf{6 a} /$ pyrrole/DPP $\mathbf{1 1}=1 / 0.5 / 1 \mathrm{mmol}$. ${ }^{\mathrm{b}}$ Yields of isolated products. ${ }^{\mathrm{c} E t h y l}$ salt $\mathbf{6 a}(\mathrm{R}=\mathrm{Et})$ was used. ${ }^{\mathrm{d}}$ Reaction was performed with

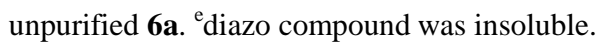

Optimization reactions were performed with $0.5 \mathrm{mmol}$ ( 1 equiv) of pyrrole, $1 \mathrm{mmol}$ ( 2 equiv) of salt 6 a and $0.5 \mathrm{mmol}$ (1 equiv) of diphenyl phosphate (DPP) 11 at $0{ }^{\circ} \mathrm{C}$ in an appropriate solvent.

Procedure for entries 1-10 and 12-14: To a suspension of 1-alkoxypyridazin-1-ium alkyl sulfate 6a $(1 \mathrm{mmol})$ in an appropriate solvent $(10 \mathrm{ml})$ at $0{ }^{\circ} \mathrm{C}$ was added cold $1 \mathrm{~N}$ aq $\mathrm{KOH}(2 \mathrm{ml})$. The contents were extracted and the organic layer was collected and concentrated to $2 \mathrm{ml}$ volume 
under reduced pressure at $0{ }^{\circ} \mathrm{C}$. The crude $(E)$-enaldiazomethane $7 \mathbf{a}$ was immediately added dropwise over $5 \mathrm{~min}$ to a solution of pyrrole $(0.5 \mathrm{mmol})$ and a rhodium catalyst in the same solvent $(2 \mathrm{ml})$ at $0{ }^{\circ} \mathrm{C}$. After $5 \mathrm{~min}$, diphenyl phosphate $(0.5 \mathrm{mmol})$ was added to the reaction mixture and continued stirring for another $10 \mathrm{~min}$. The indole product was purified by a silica gel flash chromography using ethyl acetate - petroleum ether as the eluent.

Procedure for entry 11: A mixture of pyridazine 1-oxide 9a $(1.1 \mathrm{mmol})$ and dimethyl sulfate $(1.3 \mathrm{mmol})$ in a $10 \mathrm{ml}$ round-bottom flask was stirred at $70{ }^{\circ} \mathrm{C}$ for $2 \mathrm{~h}$. The flask was cooled to 0 ${ }^{\circ} \mathrm{C}$ and the contents were extracted with ice cold $1 \mathrm{~N}$ aq KOH $(2 \mathrm{ml})$ and DCM $(10 \mathrm{ml})$. The organic layer was collected and concentrated to $2 \mathrm{ml}$ volume under reduced pressure at $0{ }^{\circ} \mathrm{C}$. The crude enaldiazomethane 7a was immediately added dropwise over 5 min to a DCM solution (2 $\mathrm{ml})$ of pyrrole $(0.5 \mathrm{mmol})$ and $\mathrm{Rh}_{2}(\mathrm{esp})_{2}(0.0015 \mathrm{mmol})$ at $0{ }^{\circ} \mathrm{C}$. After $5 \mathrm{~min}$, diphenyl phosphate $11(0.5 \mathrm{mmol})$ was added to the reaction mixture and continued stirring for another $10 \mathrm{~min}$. The crude reaction mixture was purified by a silica gel flash chromography using ethyl acetate petroleum ether as the eluent.

\section{Scope of the transannulation towards alkylindoles}
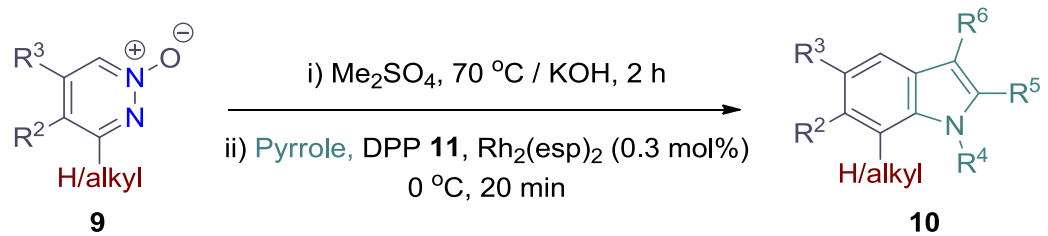

\section{General procedure D:}

A mixture of pyridazine 1-oxide $9(1.1 \mathrm{mmol})$ and dimethyl sulfate $(1.3 \mathrm{mmol})$ in a $10 \mathrm{ml}$ roundbottom flask was stirred at $70{ }^{\circ} \mathrm{C}$ for $2 \mathrm{~h}$. The flask was cooled to $0{ }^{\circ} \mathrm{C}$ and the contents were extracted with ice cold $1 \mathrm{~N}$ aq $\mathrm{KOH}(2 \mathrm{ml})$ and DCM $(10 \mathrm{ml})$. The organic layer was collected and concentrated to $2 \mathrm{ml}$ volume under reduced pressure at $0{ }^{0} \mathrm{C}$. The crude diazo enal was immediately added dropwise over $5 \mathrm{~min}$ to a DCM solution $(2 \mathrm{ml})$ of pyrrole $(0.5 \mathrm{mmol})$ and $\mathrm{Rh}_{2}(\operatorname{esp})_{2}(0.0015 \mathrm{mmol})$ at $0{ }^{\circ} \mathrm{C}$. After $5 \mathrm{~min}$, diphenyl phosphate DPP $11(0.5 \mathrm{mmol})$ was added to the reaction mixture and continued stirring for another $10 \mathrm{~min}$. The crude reaction mixture was purified by a silica gel flash chromography using ethyl acetate - petroleum ether as the eluent to furnish indole $\mathbf{1 0}$. 


\section{Spectral data for indole compounds:}

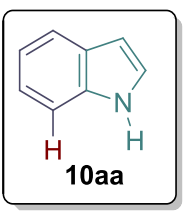

1H-Indole (10aa): Prepared by following General procedure D. Obtained as a white solid $(0.042 \mathrm{~g}$, Yield $=73 \%)$; The product data was matched with the commercially available compound. $\mathrm{R}_{\mathrm{f}}=0.4$ (Ethyl Acetate/Hexane :10/90); ${ }^{1} \mathrm{H}$ NMR $\left(400 \mathrm{MHz}, \mathrm{CDCl}_{3}\right) \delta 8.14(\mathrm{~s}, 1 \mathrm{H})$, $7.67(\mathrm{~d}, J=7.8 \mathrm{~Hz}, 1 \mathrm{H}), 7.41(\mathrm{~d}, J=8.0 \mathrm{~Hz}, 1 \mathrm{H}), 7.24-7.18(\mathrm{~m}, 2 \mathrm{H}), 7.14(\mathrm{t}, J=7.4 \mathrm{~Hz}, 1 \mathrm{H})$, $6.58(\mathrm{~s}, 1 \mathrm{H}) ;{ }^{13} \mathrm{C} \mathrm{NMR}\left(101 \mathrm{MHz}, \mathrm{CDCl}_{3}\right) \delta 135.9,128.0,124.2,122.12,120.9,119.9,111.1$, 102.8 .

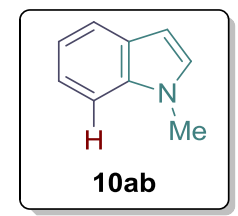

1-Methyl-1H-indole (10ab): ${ }^{10}$ Prepared by following General procedure D. Obtained as a brown liquid (0.042 g, Yield $=65 \%) ; \mathrm{R}_{\mathrm{f}}=0.5$ (Ethyl Acetate/Hexane: 10/90); Desired product data were matched with known literature compound. ${ }^{1} \mathrm{H}$ NMR $\left(400 \mathrm{MHz}, \mathrm{CDCl}_{3}\right) \delta 7.67(\mathrm{~d}, J=$ $7.9 \mathrm{~Hz}, 1 \mathrm{H}), 7.35(\mathrm{~d}, J=8.1 \mathrm{~Hz}, 1 \mathrm{H}), 7.29-7.23(\mathrm{~m}, 1 \mathrm{H}), 7.18-7.12(\mathrm{~m}, 1 \mathrm{H}), 7.07$ (d, $J=3.1$ $\mathrm{Hz}, 1 \mathrm{H}), 6.52(\mathrm{~d}, J=2.8 \mathrm{~Hz}, 1 \mathrm{H}), 3.80(\mathrm{~s}, 3 \mathrm{H}) ;{ }^{13} \mathrm{C} \mathrm{NMR}\left(101 \mathrm{MHz}, \mathrm{CDCl}_{3}\right) \delta 136.8,128.9$, $128.6,121.6,121.0,119.4,109.3,101.0,32.9$.

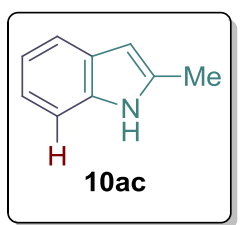

2-Methyl-1H-indole (10ac): ${ }^{11}$ Prepared by following General procedure D. Obtained as a pale yellow solid (0.045 g, Yield $=70 \%) ; \mathrm{R}_{\mathrm{f}}=0.5$ (Ethyl Acetate/Hexane: 10/90); desired product data was matched with known literature compound; ${ }^{1} \mathrm{H}$ NMR $\left(700 \mathrm{MHz}, \mathrm{CDCl}_{3}\right) \delta 7.86(\mathrm{~s}, 1 \mathrm{H})$, $7.51(\mathrm{~d}, J=7.9 \mathrm{~Hz}, 1 \mathrm{H}), 7.29(\mathrm{dd}, J=8.0,0.8 \mathrm{~Hz}, 1 \mathrm{H}), 7.12-7.09$ (m, 1H), $7.06(\mathrm{td}, J=7.5,1.0$ $\mathrm{Hz}, 1 \mathrm{H}), 6.22(\mathrm{dt}, J=1.9,0.9 \mathrm{~Hz}, 1 \mathrm{H}), 2.45(\mathrm{~d}, J=0.8 \mathrm{~Hz}, 3 \mathrm{H}) ;{ }^{13} \mathrm{C} \mathrm{NMR}\left(176 \mathrm{MHz}, \mathrm{CDCl}_{3}\right) \delta$ $136.1,135.2,129.2,121.1,119.7(2 \mathrm{C}), 110.3,100.5,13.9$. 


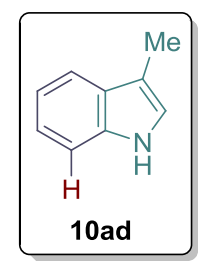

3-Methyl-1H-indole (10ad): ${ }^{11}$ Prepared by following General procedure D. Obtained as a white color solid (0.043 g, Yield $=67 \%) ; \mathrm{R}_{\mathrm{f}}=0.5$ (Ethyl Acetate/Hexane: 20/80); desired product data was matched with known literature compound; ${ }^{1} \mathrm{H} \mathrm{NMR}\left(400 \mathrm{MHz}, \mathrm{CDCl}_{3}\right) \delta 7.85$ (s, 1H), $7.61(\mathrm{~d}, J=7.7 \mathrm{~Hz}, 1 \mathrm{H}), 7.36(\mathrm{~d}, J=8.0 \mathrm{~Hz}, 1 \mathrm{H}), 7.21(\mathrm{t}, J=7.3 \mathrm{~Hz}, 1 \mathrm{H}), 7.15(\mathrm{t}, J=$ $7.2 \mathrm{~Hz}, 1 \mathrm{H}), 6.97(\mathrm{~s}, 1 \mathrm{H}), 2.36(\mathrm{~s}, 3 \mathrm{H}) ;{ }^{13} \mathrm{C} \mathrm{NMR}\left(101 \mathrm{MHz}, \mathrm{CDCl}_{3}\right) \delta 136.4,128.4,122.0$, $121.7,119.2,119.0,111.9,111.1,9.8$.

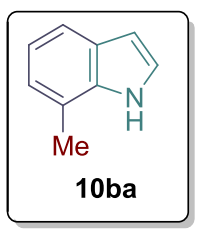

7-Methyl-1H-indole (10ba): ${ }^{12}$ Prepared by following General procedure D. Obtained as a brown solid $(0.040 \mathrm{~g}$, Yield $=62 \%) ; \mathrm{R}_{\mathrm{f}}=0.5$ (Ethyl Acetate/Hexane: $\left.20 / 80\right)$; desired product was matched with commercially available compound. ${ }^{1} \mathrm{H} \mathrm{NMR}\left(400 \mathrm{MHz}, \mathrm{CDCl}_{3}\right) \delta 8.07$ (s, $1 \mathrm{H}), 7.52(\mathrm{~d}, J=7.7 \mathrm{~Hz}, 1 \mathrm{H}), 7.22(\mathrm{t}, J=2.8 \mathrm{~Hz}, 1 \mathrm{H}), 7.06(\mathrm{t}, J=7.4 \mathrm{~Hz}, 1 \mathrm{H}), 7.01(\mathrm{~d}, J=7.0$ $\mathrm{Hz}, 1 \mathrm{H}), 6.58(\mathrm{dd}, J=3.0,2.1 \mathrm{~Hz}, 1 \mathrm{H}), 2.52(\mathrm{~s}, 3 \mathrm{H}) ;{ }^{13} \mathrm{C} \mathrm{NMR}\left(101 \mathrm{MHz}, \mathrm{CDCl}_{3}\right) \delta 135.6$, $127.5,123.9,122.6,120.3,120.1,118.6,103.3,16.8$.

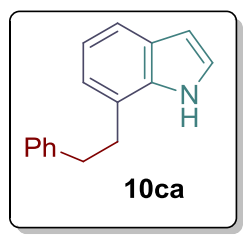

7-Phenethyl-1H-indole (10ca): Prepared by following General procedure D. Obtained brown sticky solid (0.049 g, Yield $=45 \%) ; \mathrm{R}_{\mathrm{f}}=0.4$ (ethyl acetate:100); ${ }^{1} \mathrm{H}$ NMR $\left(400 \mathrm{MHz}, \mathrm{CDCl}_{3}\right) \delta$ $7.79(\mathrm{~s}, 1 \mathrm{H}), 7.54(\mathrm{~d}, J=7.5 \mathrm{~Hz}, 1 \mathrm{H}), 7.34-7.28(\mathrm{~m}, 2 \mathrm{H}), 7.27-7.19(\mathrm{~m}, 3 \mathrm{H}), 7.13-7.04(\mathrm{~m}$, $3 \mathrm{H}), 6.55(\mathrm{dd}, J=3.1,2.1 \mathrm{~Hz}, 1 \mathrm{H}), 3.19-3.13(\mathrm{~m}, 2 \mathrm{H}), 3.11-3.04(\mathrm{~m}, 2 \mathrm{H}) ;{ }^{13} \mathrm{C} \mathrm{NMR}(101$ $\left.\mathrm{MHz}, \mathrm{CDCl}_{3}\right) \delta 142.1,135.1,128.7,128.6,127.8,126.3,124.3,124.1,121.6,120.2,118.9$, 103.1, 36.4, 33.7; IR (neat) :3427, 2924, 2864, 1602, 1494, 1433, 1106, 790, $727 \mathrm{~cm}^{-1}$; HRMS (ESI) $\mathrm{m} /$ zcalc. for $\mathrm{C}_{16} \mathrm{H}_{15} \mathrm{~N}(\mathrm{M}+\mathrm{H})^{+} 222.1277$, found 222.1289 . 


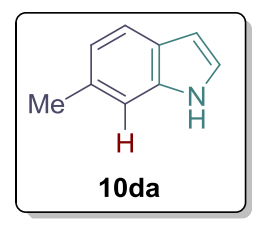

6-Methyl-1H-indole (10da): ${ }^{13}$ Prepared by following General procedure D. Obtained as a pale white solid (0.036 g, Yield $=55 \%) ; R_{\mathrm{f}}=0.4$ (Ethyl Acetate/Hexane: 10/90); The desired product data were matched with reported compound. ${ }^{1} \mathrm{H}$ NMR $\left(400 \mathrm{MHz}, \mathrm{CDCl}_{3}\right) \delta 7.93(\mathrm{~s}, 1 \mathrm{H}), 7.58(\mathrm{~d}$, $J=8.0 \mathrm{~Hz}, 1 \mathrm{H}), 7.19$ (s, 1H), $7.15-7.11(\mathrm{~m}, 1 \mathrm{H}), 7.01(\mathrm{~d}, J=8.0 \mathrm{~Hz}, 1 \mathrm{H}), 6.55(\mathrm{~s}, 1 \mathrm{H}), 2.52(\mathrm{~s}$, $3 \mathrm{H}) ;{ }^{13} \mathrm{C}$ NMR $\left(101 \mathrm{MHz}, \mathrm{CDCl}_{3}\right) \delta 136.4,131.9,125.7,123.6,121.7,120.4,111.1,102.5,21.8$.

\section{Scope of the transannulation towards 7-arylindoles}
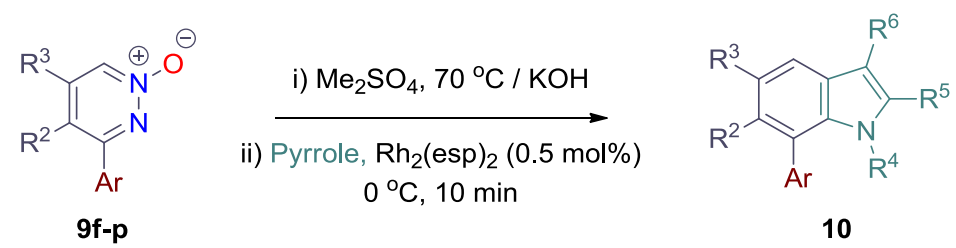

General procedure E: A mixture of 3-arylpyridazine 1-oxide 9f-p (0.93 mmol) and dimethyl sulphate $(1.11 \mathrm{mmol})$ in a $10 \mathrm{ml}$ round-bottom flask was stirred at $70{ }^{\circ} \mathrm{C}$ for $2 \mathrm{~h}$. The flask was cooled to $0{ }^{\circ} \mathrm{C}$ and the contents were extracted with ice cold $1 \mathrm{~N}$ aq $\mathrm{KOH}(3 \mathrm{ml})$ and DCM (10 $\mathrm{ml})$. The organic layer was collected and concentrated to $2 \mathrm{ml}$ volume under reduced pressure at $0{ }^{0} \mathrm{C}$. The crude diazo enal was immediately added dropwise over 5 min to a DCM solution (2 $\mathrm{ml})$ of pyrrole $(0.37 \mathrm{mmol})$ and $\mathrm{Rh}_{2}(\mathrm{esp})_{2}(0.00185 \mathrm{mmol})$ at $0{ }^{\circ} \mathrm{C}$. After $5 \mathrm{~min}$, the crude indole was purified by a silica gel flash chromography using ethyl acetate - petroleum ether as the eluent.

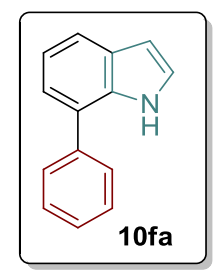

7-Phenyl-1H-indole (10fa): ${ }^{14}$ Prepared by following General procedure E. Obtained as a yellow semi solid $(0.045 \mathrm{~g}$, Yield $=63 \%) ; \mathrm{R}_{\mathrm{f}}=0.45$ (Ethyl Acetate/Hexane : 10/90); ${ }^{1} \mathrm{H}$ NMR $\left(400 \mathrm{MHz}, \mathrm{CDCl}_{3}\right) \delta 8.48(\mathrm{~s}, 1 \mathrm{H}), 7.72(\mathrm{dd}, J=6.0,2.0 \mathrm{~Hz}, 3 \mathrm{H}), 7.59$ (dd, $\left.J=10.5,4.7 \mathrm{~Hz}, 2 \mathrm{H}\right)$, 
$7.47(\mathrm{t}, J=7.4 \mathrm{~Hz}, 1 \mathrm{H}), 7.31-7.26(\mathrm{~m}, 3 \mathrm{H}), 6.70(\mathrm{dd}, J=3.1,2.1 \mathrm{~Hz}, 1 \mathrm{H}) ;{ }^{13} \mathrm{C}$ NMR $(101$ $\left.\mathrm{MHz}, \mathrm{CDCl}_{3}\right) \delta 139.3,133.7,129.2,128.3,128.2,127.4,125.6,124.3,121.9,120.3,120.0$, 103.1; IR (Neat): 3440, 1604, 1518, 1484, 1418, 1336, $759 \mathrm{~cm}^{-1}$; HRMS (ESI) $\mathrm{m} / z$ calc. for $\mathrm{C}_{14} \mathrm{H}_{11} \mathrm{~N}(\mathrm{M}+\mathrm{H})^{+}$194.0964, found 194.0956.

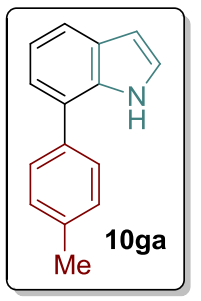

7-(p-Tolyl)-1H-indole (10ga): Prepared by following General procedure E. Obtained as a liquid (0.045 g, Yield $=60 \%) ; \mathrm{R}_{\mathrm{f}}=0.45$ (ethyl acetate/hexane : 20:80); ${ }^{1} \mathrm{H}$ NMR $(400 \mathrm{MHz}$, $\left.\mathrm{CDCl}_{3}\right) \delta 8.41(\mathrm{~s}, 1 \mathrm{H}), 7.65(\mathrm{dd}, J=5.6,3.3 \mathrm{~Hz}, 1 \mathrm{H}), 7.56(\mathrm{~d}, J=8.0 \mathrm{~Hz}, 2 \mathrm{H}), 7.34(\mathrm{~d}, J=7.8$ $\mathrm{Hz}, 2 \mathrm{H}), 7.25-7.20$ (m, 3H), $6.64(\mathrm{dd}, J=3.1,2.1 \mathrm{~Hz}, 1 \mathrm{H}), 2.46(\mathrm{~s}, 3 \mathrm{H}) ;{ }^{13} \mathrm{C}$ NMR $(101 \mathrm{MHz}$, $\left.\mathrm{CDCl}_{3}\right) \delta 137.3,136.5,133.9,130.0,128.4,128.2,125.7,124.4,121.9,120.4,119.9,103.2,21.3$; IR (neat): 3432, 3055, 2921, 2864, 1614, 1485, 1333, 1113, 926, $793 \mathrm{~cm}^{-1}$; HRMS (ESI) $\mathrm{m} / \mathrm{z}$ calc. for $\mathrm{C}_{15} \mathrm{H}_{13} \mathrm{~N}(\mathrm{M}+\mathrm{H})^{+} 208.1121$, found 208.1118.

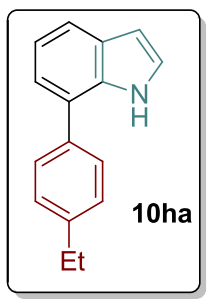

7-(4-Ethylphenyl)-1H-indole (10ha): Prepared by following General procedure E. Obtained as a white solid $(0.047 \mathrm{~g}$, Yield $=58 \%)$; m.p. $=52-54{ }^{0} \mathrm{C} ; \mathrm{R}_{\mathrm{f}}=0.4$ (Ethyl Acetate/Hexane : 10/90); ${ }^{1} \mathrm{H}$ NMR $\left(400 \mathrm{MHz}, \mathrm{CDCl}_{3}\right) \delta 8.43(\mathrm{~s}, 1 \mathrm{H}), 7.64(\mathrm{dd}, J=6.3,2.4 \mathrm{~Hz}, 1 \mathrm{H}), 7.57(\mathrm{~d}, J=$ $8.1 \mathrm{~Hz}, 2 \mathrm{H}), 7.35(\mathrm{~d}, J=8.1 \mathrm{~Hz}, 2 \mathrm{H}), 7.24-7.19(\mathrm{~m}, 3 \mathrm{H}), 6.63(\mathrm{dd}, J=3.1,2.1 \mathrm{~Hz}, 1 \mathrm{H}), 2.74$ (q, $J=7.6 \mathrm{~Hz}, 2 \mathrm{H}), 1.32$ (t, $J=7.6 \mathrm{~Hz}, 3 \mathrm{H}) ;{ }^{13} \mathrm{C} \mathrm{NMR}\left(101 \mathrm{MHz}, \mathrm{CDCl}_{3}\right) \delta 143.7,136.7,133.9$, 128.0, 128.4, 128.3, 125.8, 124.4, 121.9, 120.4, 119.9, 103.2, 28.8, 15.7; IR (Neat): 3440, 1629, 1413, 1335, 1270, 1065, 796, $729 \mathrm{~cm}^{-1}$; HRMS (ESI) $\mathrm{m} / z$ calc. for $\mathrm{C}_{16} \mathrm{H}_{15} \mathrm{~N}(\mathrm{M}+\mathrm{H})^{+} 222.1277$, found 222.1289 . 


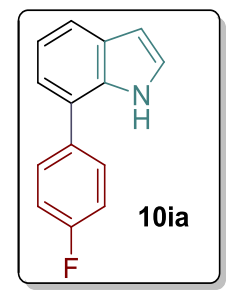

7-(4-Fluorophenyl)-1H-indole (10ia): Prepared by following General procedure E. Obtained as a white solid $(0.042 \mathrm{~g}$, Yield $=55 \%) ;$ m.p. $=72-74{ }^{0} \mathrm{C} ; \mathrm{R}_{\mathrm{f}}=0.4($ Ethyl Acetate $/$ Hexane :10/90); ${ }^{1} \mathrm{H}$ NMR (400 MHz, $\left.\mathrm{CDCl}_{3}\right) \delta 8.31(\mathrm{~s}, 1 \mathrm{H}), 7.66(\mathrm{~d}, J=7.2 \mathrm{~Hz}, 1 \mathrm{H}), 7.59$ (dd, $J=8.0$, $5.7 \mathrm{~Hz}, 2 \mathrm{H}), 7.26-7.15(\mathrm{~m}, 5 \mathrm{H}), 6.64(\mathrm{~s}, 1 \mathrm{H}) ;{ }^{13} \mathrm{C} \mathrm{NMR}\left(101 \mathrm{MHz}, \mathrm{CDCl}_{3}\right) \delta 163.6$ and 161.1 $(\mathrm{d}, J=246.6 \mathrm{~Hz}), 135.4$ and $135.4(\mathrm{~d}, J=3.3 \mathrm{~Hz}), 133.8,130.0,129.9$ and $128.5(\mathrm{~d}, J=7.9 \mathrm{~Hz})$, 124.7, 124.6, 122.1, 120.4, 120.3, 116.3 and 116.1 (d, $J=21.4 \mathrm{~Hz}), 103.3$; IR (Neat): 3440, 1612, 1504, 1414, 1224, 1095, 841, $730 \mathrm{~cm}^{-1}$; HRMS (ESI) $\mathrm{m} / z$ calc. for $\mathrm{C}_{14} \mathrm{H}_{10} \mathrm{FN}(\mathrm{M}+\mathrm{H})^{+}$ 212.0870, found 212.0877 .

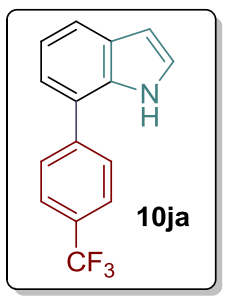

7-(4-(Trifluoromethyl)phenyl)-1H-indole (10ja): Prepared by following General procedure E. Obtained as a white solid $(0.057 \mathrm{~g}$, Yield $=60 \%)$; m.p. $=116-118{ }^{0} \mathrm{C} ; \mathrm{R}_{\mathrm{f}}=0.4$ (Ethyl Acetate/Hexane : 10/90); ${ }^{1} \mathrm{H}$ NMR (400 MHz, $\left.\mathrm{CDCl}_{3}\right) \delta 8.35(\mathrm{~s}, 1 \mathrm{H}), 7.80-7.73(\mathrm{~m}, 4 \mathrm{H}), 7.73-$ $7.67(\mathrm{~m}, 1 \mathrm{H}), 7.25-7.21(\mathrm{~m}, 3 \mathrm{H}), 6.68-6.64(\mathrm{~m}, 1 \mathrm{H}) ;{ }^{13} \mathrm{C} \mathrm{NMR}\left(101 \mathrm{MHz}, \mathrm{CDCl}_{3}\right) \delta 143.0$, 133.6, 129.8, 129.5, 128.7, 128.7, 126.2 (q, $J=3.8 \mathrm{~Hz}), 124.8,124.2,122.3$, 121.1, 120.6, 103.5; IR (Neat): 3417, 1626, 1380, 1324, 1164, 1117, 1096, 800, $737 \mathrm{~cm}^{-1}$; HRMS (Q-TOF) m/z calc. for $\mathrm{C}_{15} \mathrm{H}_{10} \mathrm{~F}_{3} \mathrm{~N}(\mathrm{M})^{+}$261.0739, found 261.0731.

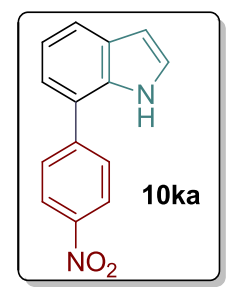


7-(4-Nitrophenyl)-1H-indole (10ka): Prepared by following General procedure E. Obtained as a white solid $(0.059 \mathrm{~g}$, Yield $=68 \%)$; m.p. $=196-198{ }^{0} \mathrm{C} ; \mathrm{R}_{\mathrm{f}}=0.4$ (Ethyl Acetate/Hexane : 10/90); ${ }^{1} \mathrm{H}$ NMR (500 MHz, $\left.\mathrm{CDCl}_{3}\right) \delta 8.43(\mathrm{~s}, 1 \mathrm{H}), 8.42-8.38(\mathrm{~m}, 2 \mathrm{H}), 7.87-7.83(\mathrm{~m}, 2 \mathrm{H})$, $7.76(\mathrm{ddd}, J=6.0,3.0,0.5 \mathrm{~Hz}, 1 \mathrm{H}), 7.32-7.30(\mathrm{~m}, 1 \mathrm{H}), 7.29-7.27(\mathrm{~m}, 2 \mathrm{H}), 6.70(\mathrm{dd}, J=3.2$, $2.0 \mathrm{~Hz}, 1 \mathrm{H}) ;{ }^{13} \mathrm{C}$ NMR $\left(126 \mathrm{MHz}, \mathrm{CDCl}_{3}\right) \delta 147.1,146.4,133.4,129.0,128.9,125.0,124.6$, 123.3, 122.5, 121.9, 120.6, 103.7; IR (Neat): 3398, 2922, 2862, 1591, 1504, 1340, 1267, 1022, $756 \mathrm{~cm}^{-1}$; HRMS (ESI) $\mathrm{m} / z$ calc. for $\mathrm{C}_{14} \mathrm{H}_{10} \mathrm{~N}_{2} \mathrm{O}_{2}(\mathrm{M}+\mathrm{H})^{+} 239.0815$, found 239.0827.

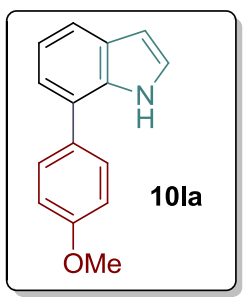

7-(4-Methoxyphenyl)-1H-indole (10la): Prepared by following General procedure E. Obtained as a white solid $(0.042 \mathrm{~g}$, Yield $=52 \%) ;$ m.p. $=112-114{ }^{0} \mathrm{C} ; \mathrm{R}_{\mathrm{f}}=0.4$ (Ethyl Acetate/Hexane : 10/90); ${ }^{1} \mathrm{H}$ NMR $\left(500 \mathrm{MHz}, \mathrm{CDCl}_{3}\right) \delta 8.39(\mathrm{~s}, 1 \mathrm{H}), 7.66-7.62(\mathrm{~m}, 1 \mathrm{H}), 7.60$ $7.56(\mathrm{~m}, 2 \mathrm{H}), 7.23-7.18(\mathrm{~m}, 3 \mathrm{H}), 7.08-7.04(\mathrm{~m}, 2 \mathrm{H}), 6.63(\mathrm{dd}, J=3.2,2.1 \mathrm{~Hz}, 1 \mathrm{H}), 3.89$ (s, $3 \mathrm{H}) ;{ }^{13} \mathrm{C} \mathrm{NMR}\left(101 \mathrm{MHz}, \mathrm{CDCl}_{3}\right) \delta 159.2,134.0,131.8,129.4,128.3,125.5,124.4,121.8$, 120.4, 119.7, 114.7, 103.2, 55.6; IR (Neat): 3432, 1628, 1586, 1280, 1085, 1038, 795, $740 \mathrm{~cm}^{-1}$; HRMS (ESI) $m / z$ calc. for $\mathrm{C}_{15} \mathrm{H}_{13} \mathrm{NO}(\mathrm{M}+\mathrm{H})^{+} 224.1070$, found 224.1060.

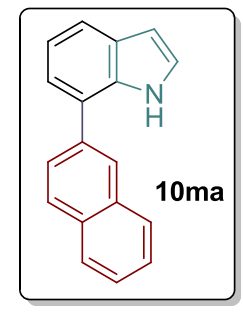

7-(Naphthalen-2-yl)-1H-indole (10ma): Prepared by following General procedure E. Obtained as a brown solid $(0.055 \mathrm{~g}$, Yield $=62 \%) ; \mathrm{m} . \mathrm{p}=98-100{ }^{0} \mathrm{C} ; \mathrm{R}_{\mathrm{f}}=0.4$ (Ethyl Acetate/Hexane :10/90); ${ }^{1} \mathrm{H}$ NMR $\left(500 \mathrm{MHz}, \mathrm{CDCl}_{3}\right) \delta 8.48(\mathrm{~s}, 1 \mathrm{H}), 8.11(\mathrm{~d}, J=0.8 \mathrm{~Hz}, 1 \mathrm{H})$, $8.00(\mathrm{~d}, J=8.4 \mathrm{~Hz}, 1 \mathrm{H}), 7.95-7.90(\mathrm{~m}, 2 \mathrm{H}), 7.78(\mathrm{dd}, J=8.4,1.7 \mathrm{~Hz}, 1 \mathrm{H}), 7.71(\mathrm{~d}, J=7.9 \mathrm{~Hz}$, $1 \mathrm{H}), 7.59-7.52(\mathrm{~m}, 2 \mathrm{H}), 7.35(\mathrm{dd}, J=7.2,1.0 \mathrm{~Hz}, 1 \mathrm{H}), 7.28(\mathrm{~d}, J=7.8 \mathrm{~Hz}, 1 \mathrm{H}), 7.25-7.23(\mathrm{~m}$, $1 \mathrm{H}), 6.68(\mathrm{dd}, J=3.2,2.1 \mathrm{~Hz}, 1 \mathrm{H}) ;{ }^{13} \mathrm{C} \mathrm{NMR}\left(101 \mathrm{MHz}, \mathrm{CDCl}_{3}\right) \delta 136.8,134.1,133.9,132.8$, 
129.0, 128.5, 128.1, 127.9, 126.9, 126.8, 126.7, 126.2, 125.7, 124.6, 122.3, 120.5, 120.3, 103.3; IR (Neat) : 3416, 1596, 1316, 1095, 795, $745 \mathrm{~cm}^{-1}$; HRMS (ESI) $\mathrm{m} / z$ calc. for $\mathrm{C}_{18} \mathrm{H}_{13} \mathrm{~N}(\mathrm{M}+\mathrm{H})^{+}$ 244.1121, found 244.1122 .

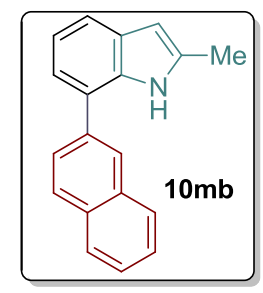

2-Methyl-7-(naphthalen-2-yl)-1H-indole (10mb): Prepared by following General procedure E. Obtained as a brown solid $(0.059 \mathrm{~g}$, Yield $=63 \%)$; m.p. $=126-128{ }^{0} \mathrm{C} ; \mathrm{R}_{\mathrm{f}}=0.4$ (Ethyl Acetate/Hexane : 10/90); ${ }^{1} \mathrm{H}$ NMR $\left(400 \mathrm{MHz}, \mathrm{CDCl}_{3}\right) \delta 8.08(\mathrm{~s}, 1 \mathrm{H}), 8.01(\mathrm{~s}, 1 \mathrm{H}), 7.91(\mathrm{~d}, J=$ $8.4 \mathrm{~Hz}, 1 \mathrm{H}), 7.83(\mathrm{~d}, J=9.3 \mathrm{~Hz}, 2 \mathrm{H}), 7.68(\mathrm{dd}, J=8.4,1.6 \mathrm{~Hz}, 1 \mathrm{H}), 7.50-7.43(\mathrm{~m}, 3 \mathrm{H}), 7.18-$ $7.09(\mathrm{~m}, 2 \mathrm{H}), 6.24(\mathrm{~d}, J=0.9 \mathrm{~Hz}, 1 \mathrm{H}), 2.37(\mathrm{~s}, 3 \mathrm{H}) ;{ }^{13} \mathrm{C} \mathrm{NMR}\left(101 \mathrm{MHz}, \mathrm{CDCl}_{3}\right) \delta$ 137.1, 135.6, 134.2, 133.9, 132.7, 129.7, 129.0, 128.1, 127.9, 126.9, 126.9, 126.6, 126.2, 124.9, 121.4, 120.3, 119.2, 101.1, 13.9; IR (Neat): 3440, 1623, 1331, 1280, 859, 795, $743 \mathrm{~cm}^{-1}$; HRMS (ESI) $\mathrm{m} / \mathrm{z}$ calc. for $\mathrm{C}_{19} \mathrm{H}_{15} \mathrm{~N}(\mathrm{M}+\mathrm{H})^{+} 258.127$, found 258.1289.

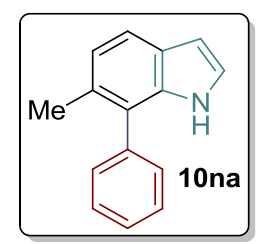

6-Methyl-7-phenyl-1H-indole (10na): Prepared by following General procedure E. Obtained as a brown liquid $(0.026 \mathrm{~g}$, Yield $=35 \%) ; \mathrm{R}_{\mathrm{f}}=0.4$ (Ethyl Acetate/Hexane : 10/90); ${ }^{1} \mathrm{H}$ NMR $\left(400 \mathrm{MHz}, \mathrm{CDCl}_{3}\right) \delta 7.87(\mathrm{~s}, 1 \mathrm{H}), 7.55-7.48(\mathrm{~m}, 3 \mathrm{H}), 7.44-7.39(\mathrm{~m}, 3 \mathrm{H}), 7.09(\mathrm{~d}, J=3.0 \mathrm{~Hz}$, $1 \mathrm{H}), 7.07(\mathrm{~d}, J=5.5 \mathrm{~Hz}, 1 \mathrm{H}), 6.54(\mathrm{dd}, J=3.2,2.1 \mathrm{~Hz}, 1 \mathrm{H}), 2.30(\mathrm{~s}, 3 \mathrm{H}) ;{ }^{13} \mathrm{C} \mathrm{NMR}(101 \mathrm{MHz}$, $\left.\mathrm{CDCl}_{3}\right) \delta 138.0,135.5,129.9,129.0,128.8,127.5,125.9,124.8,123.8,122.8,119.6,102.8,19.7$; IR(neat) : 3431, 1630, 1580, 1285, 1085, 810; HRMS (ESI) $\mathrm{m} / z$ calc. for $\mathrm{C}_{15} \mathrm{H}_{13} \mathrm{~N}(\mathrm{M}+\mathrm{H})^{+}$ 208.1121, found 208.1116 . 


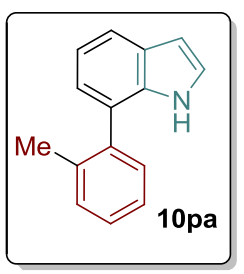

7-(o-Tolyl)-1H-indole (10pa): Prepared by following General procedure E. Obtained as a white semi solid $(0.045 \mathrm{~g}$, Yield $=60 \%) ; \mathrm{R}_{\mathrm{f}}=0.4$ (Ethyl Acetate/Hexane $\left.: 10 / 90\right) ;{ }^{1} \mathrm{H}$ NMR $(400$ $\left.\mathrm{MHz} \mathrm{CDCl}_{3}\right) \delta 7.90(\mathrm{~s}, 1 \mathrm{H}), 7.67(\mathrm{~d}, J=7.9 \mathrm{~Hz}, 1 \mathrm{H}), 7.41-7.28(\mathrm{~m}, 4 \mathrm{H}), 7.21(\mathrm{t}, J=7.5 \mathrm{~Hz}$, 1H), 7.15 (t, $J=2.8 \mathrm{~Hz}, 1 \mathrm{H}), 7.09$ (d, $J=7.1 \mathrm{~Hz}, 1 \mathrm{H}), 6.63$ (dd, $J=3.0,2.2 \mathrm{~Hz}, 1 \mathrm{H}), 2.20$ (s, $3 \mathrm{H}) ;{ }^{13} \mathrm{C}$ NMR $\left(101 \mathrm{MHz}, \mathrm{CDCl}_{3}\right) \delta 138.3,136.7,134.5,130.7,130.1,127.9,127.9,126.1$, 125.4, 124.3, 122.7, 119.9, 119.8, 102.9, 20.1; IR (Neat): 3415, 1604, 1483, 1424, 1332, 1272, $800 \mathrm{~cm}^{-1}$; HRMS (ESI) $\mathrm{m} / z$ calc. for $\mathrm{C}_{15} \mathrm{H}_{13} \mathrm{~N}(\mathrm{M}+\mathrm{H})^{+} 208.1121$, found 208.1133 .

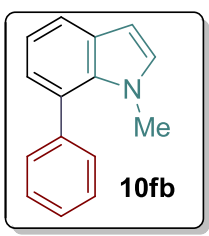

1-Methyl-7-phenyl-1H-indole (10fb): Prepared by following General procedure E. Obtained as a white solid $(0.036 \mathrm{~g}$, Yield $=48 \%) ; \mathrm{m} . \mathrm{p} .=42-44{ }^{0} \mathrm{C} ; \mathrm{R}_{\mathrm{f}}=0.3$ (Ethyl Acetate/Hexane: 10/90); ${ }^{1} \mathrm{H}$ NMR $\left(400 \mathrm{MHz}, \mathrm{CDCl}_{3}\right) \delta 7.63(\mathrm{dd}, J=7.9,1.1 \mathrm{~Hz}, 1 \mathrm{H}), 7.48-7.37$ (m, 5H), 7.12 $(\mathrm{t}, J=7.5 \mathrm{~Hz}, 1 \mathrm{H}), 7.03(\mathrm{dd}, J=7.1,1.0 \mathrm{~Hz}, 1 \mathrm{H}), 6.98(\mathrm{~d}, J=3.1 \mathrm{~Hz}, 1 \mathrm{H}), 6.55(\mathrm{~d}, J=3.1 \mathrm{~Hz}$, 1H), $3.31(\mathrm{~s}, 3 \mathrm{H}) ;{ }^{13} \mathrm{C}$ NMR (101 MHz, $\left.\mathrm{CDCl}_{3}\right) \delta 140.6,134.1,131.0,130.3,129.8,127.7$, 127.2, 126.9, 124.3, 120.3, 119.2, 101.3, 36.9; IR (Neat): 2948, 2918, 1521, 1481, 1445, 1316, 1095, 796, 762, $595 \mathrm{~cm}^{-1}$; HRMS (ESI) $\mathrm{m} / z$ calc. for $\mathrm{C}_{15} \mathrm{H}_{13} \mathrm{~N}(\mathrm{M}+\mathrm{H})^{+}$208.1121, found 208.1113.

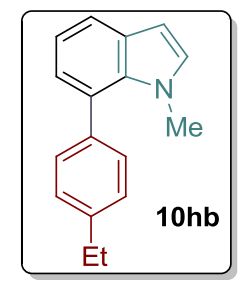

7-(4-Ethylphenyl)-1-methyl-1H-indole (10hb): Prepared by following General procedure E. Obtained as a white semi solid $(0.039 \mathrm{~g}$, Yield $=45 \%) ; \mathrm{R}_{\mathrm{f}}=0.5$ (Ethyl Acetate/Hexane: 10/90); 
${ }^{1} \mathrm{H}$ NMR $\left(400 \mathrm{MHz}, \mathrm{CDCl}_{3}\right) \delta 7.54(\mathrm{dd}, J=7.8,1.0 \mathrm{~Hz}, 1 \mathrm{H}), 7.28(\mathrm{~d}, J=8.0 \mathrm{~Hz}, 2 \mathrm{H}), 7.18(\mathrm{~d}, J$ $=8.6 \mathrm{~Hz}, 2 \mathrm{H}), 7.03(\mathrm{t}, J=7.5 \mathrm{~Hz}, 1 \mathrm{H}), 6.94(\mathrm{dd}, J=7.1,1.0 \mathrm{~Hz}, 1 \mathrm{H}), 6.90(\mathrm{~d}, J=3.1 \mathrm{~Hz}, 1 \mathrm{H})$, $6.47(\mathrm{~d}, J=3.1 \mathrm{~Hz}, 1 \mathrm{H}), 3.25$ (s, 3H), $2.66(\mathrm{q}, J=7.6 \mathrm{~Hz}, 2 \mathrm{H}), 1.24(\mathrm{t}, J=7.6 \mathrm{~Hz}, 3 \mathrm{H}) ;{ }^{13} \mathrm{C}$ NMR $\left(101 \mathrm{MHz}, \mathrm{CDCl}_{3}\right) \delta 143.3,137.8,131.0,130.2,129.8,127.2,127.0,124.4,120.1,119.2$, 101.2, 36.9, 28.7, 15.7; IR (Neat): 2963, 2921, 1519, 1445, 1313, 1094, $745 \mathrm{~cm}^{-1}$; HRMS (ESI) $m / z$ calc. for $\mathrm{C}_{17} \mathrm{H}_{17} \mathrm{~N}(\mathrm{M}+\mathrm{H})^{+} 236.1434$, found 236.1454 .

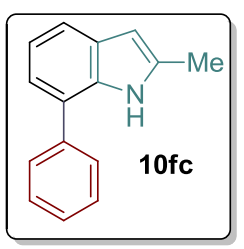

2-Methyl-7-phenyl-1H-indole (10fc): Prepared by following General procedure E. Obtained as a brown solid $(0.045 \mathrm{~g}$, Yield $=59 \%)$; m.p. $=96-98{ }^{\circ} \mathrm{C} ; \mathrm{R}_{\mathrm{f}}=0.4$ (Ethyl Acetate/Hexane: 10/90); ${ }^{1} \mathrm{H}$ NMR (400 MHz, $\left.\mathrm{CDCl}_{3}\right) \delta 8.12(\mathrm{~s}, 1 \mathrm{H}), 7.70-7.64(\mathrm{~m}, 2 \mathrm{H}), 7.58-7.51(\mathrm{~m}, 3 \mathrm{H})$, $7.43(\mathrm{t}, J=7.4 \mathrm{~Hz}, 1 \mathrm{H}), 7.21-7.14(\mathrm{~m}, 2 \mathrm{H}), 6.32(\mathrm{~d}, J=1.0 \mathrm{~Hz}, 1 \mathrm{H}), 2.46(\mathrm{~s}, 3 \mathrm{H}) ;{ }^{13} \mathrm{C}$ NMR $\left(101 \mathrm{MHz}, \mathrm{CDCl}_{3}\right) \delta 139.6,135.5,134.0,129.6,129.2,128.4,127.4,124.9,121.1,120.2,119.1$, 101.0, 13.9; IR (Neat): 3439, 1556, 1422, 1394, 1281, 802, $745 \mathrm{~cm}^{-1}$; HRMS (ESI) $\mathrm{m} / z$ calc. for $\mathrm{C}_{15} \mathrm{H}_{13} \mathrm{~N} \quad(\mathrm{M}+\mathrm{H})^{+}$208.1121, found 208.1130. CCDC 1420660 contains the X-ray crystallographic data of this compound.

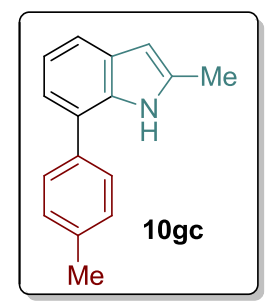

2-Methyl-7-(p-tolyl)-1H-indole (10gc): Prepared by following General procedure E. Obtained as a white solid $(0.049 \mathrm{~g}$, Yield $=60 \%) ; \mathrm{m} . \mathrm{p} .=86-90{ }^{\circ} \mathrm{C} ; \mathrm{R}_{\mathrm{f}}=0.5$ (Ethyl Acetate/Hexane : 20/80); ${ }^{1} \mathrm{H}$ NMR (400 MHz, CDCl $) \delta 8.09$ (s, $\left.1 \mathrm{H}\right), 7.54$ (d, $\left.J=8.0 \mathrm{~Hz}, 2 \mathrm{H}\right), 7.49$ (dd, $J=7.2$, $0.9 \mathrm{~Hz}, 1 \mathrm{H}), 7.33(\mathrm{~d}, J=7.8 \mathrm{~Hz}, 2 \mathrm{H}), 7.17-7.10(\mathrm{~m}, 2 \mathrm{H}), 6.28(\mathrm{~d}, J=0.8 \mathrm{~Hz}, 1 \mathrm{H}), 2.45(\mathrm{~s}, 3 \mathrm{H})$, $2.44(\mathrm{~s}, 3 \mathrm{H}) ;{ }^{13} \mathrm{C}$ NMR $\left(101 \mathrm{MHz}, \mathrm{CDCl}_{3}\right) \delta 137.2,136.7,135.4,134.1,129.9,129.5,128.3$, 124.9, 121.0, 120.2, 118.9, 101.0, 21.4, 13.9; IR (Neat): 3433, 3415, 2920, 2854, 1614, 1566, 1429, 1280, 1020, 796, $744 \mathrm{~cm}^{-1}$; HRMS (ESI) $\mathrm{m} / z$ calc. for $\mathrm{C}_{16} \mathrm{H}_{15} \mathrm{~N}(\mathrm{M}+\mathrm{H})^{+} 222.1277$, found 222.1289 . 


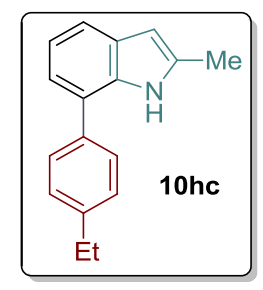

7-(4-Ethylphenyl)-2-methyl-1H-indole (10hc): Prepared by following General procedure E. Obtained as a semi solid (0.048 g, Yield = 56\%); $\mathrm{R}_{\mathrm{f}}=0.45$ (Ethyl Acetate/Hexane: 10/90); ${ }^{1} \mathrm{H}$ NMR $\left(400 \mathrm{MHz}, \mathrm{CDCl}_{3}\right) \delta 8.11(\mathrm{~s}, 1 \mathrm{H}), 7.56(\mathrm{~d}, J=8.0 \mathrm{~Hz}, 2 \mathrm{H}), 7.49(\mathrm{dd}, J=7.0,1.4 \mathrm{~Hz}, 1 \mathrm{H})$, $7.35(\mathrm{~d}, J=7.9 \mathrm{~Hz}, 2 \mathrm{H}), 7.18-7.10(\mathrm{~m}, 2 \mathrm{H}), 6.28(\mathrm{~s}, 1 \mathrm{H}), 2.74(\mathrm{q}, J=7.6 \mathrm{~Hz}, 2 \mathrm{H}), 2.44(\mathrm{~s}, 3 \mathrm{H})$, $1.32(\mathrm{t}, J=7.7 \mathrm{~Hz}, 3 \mathrm{H}) ;{ }^{13} \mathrm{C}$ NMR $\left(101 \mathrm{MHz}, \mathrm{CDCl}_{3}\right) \delta 143.5,136.9,135.4,134.0,129.5,128.7$, 128.3, 125.0, 121.0, 120.2, 118.8, 101.0, 28.8, 15.7, 13.9; IR (Neat): 3416, 1589, 1262, 1046, 798, $745 \mathrm{~cm}^{-1}$; HRMS (ESI) $\mathrm{m} / z$ calc. for $\mathrm{C}_{17} \mathrm{H}_{17} \mathrm{~N}(\mathrm{M}+\mathrm{H})^{+} 236.1434$, found 236.1435 .

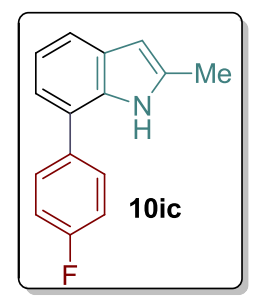

7-(4-Fluorophenyl)-2-methyl-1H-indole (10ic): Prepared by following General procedure E. Obtained as a yellow semi solid (0.051 g, Yield $=62 \%) ; \mathrm{R}_{\mathrm{f}}=0.4$ (Ethyl Acetate/Hexane: 10/90), ${ }^{1} \mathrm{H}$ NMR $\left(400 \mathrm{MHz}, \mathrm{CDCl}_{3}\right) \delta 8.01(\mathrm{~s}, 1 \mathrm{H}), 7.63-7.56(\mathrm{~m}, 2 \mathrm{H}), 7.51(\mathrm{~d}, J=7.7 \mathrm{~Hz}, 1 \mathrm{H}), 7.24-$ $7.17(\mathrm{~m}, 2 \mathrm{H}), 7.15(\mathrm{t}, J=7.5 \mathrm{~Hz}, 1 \mathrm{H}), 7.08(\mathrm{dd}, J=7.2,0.8 \mathrm{~Hz}, 1 \mathrm{H}), 6.30(\mathrm{~d}, J=0.9 \mathrm{~Hz}, 1 \mathrm{H})$, $2.45(\mathrm{~s}, 3 \mathrm{H}) ;{ }^{13} \mathrm{C} \mathrm{NMR}\left(101 \mathrm{MHz}, \mathrm{CDCl}_{3}\right) \delta 163.6$ and $161.1(\mathrm{~d}, J=246.4 \mathrm{~Hz}), 135.6,135.6$, 134.0, 130.0 and $129.9(\mathrm{~d}, J=7.9 \mathrm{~Hz}), 129.6,123.9,121.1,120.3,119.2,116.3$ and $116.0(\mathrm{~d}, J=$ 21.3 Hz), 101.2, 13.9; IR (Neat): 3439, 2920, 1695, 1603, 1510, 1224, 840, $798 \mathrm{~cm}^{1}$; HRMS (ESI) $m / z$ calc. for $\mathrm{C}_{15} \mathrm{H}_{12} \mathrm{FN}(\mathrm{M}+\mathrm{H})^{+} 226.1027$, found 226.1037 .

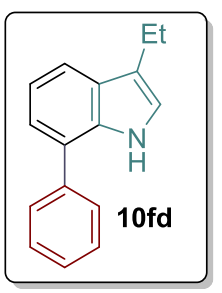


3-Ethyl-7-phenyl-1H-indole (10fd): Prepared by following General procedure E. Obtained as a viscous liquid $(0.045 \mathrm{~g}$, Yield $=56 \%) ; \mathrm{R}_{\mathrm{f}}=0.45$ (Ethyl Acetate/Hexane $\left.: 10 / 90\right) ;{ }^{1} \mathrm{H}$ NMR $(400$ $\left.\mathrm{MHz} \mathrm{CDCl}_{3}\right) \delta 8.16(\mathrm{~s}, 1 \mathrm{H}), 7.68-7.59(\mathrm{~m}, 3 \mathrm{H}), 7.50(\mathrm{t}, J=7.6 \mathrm{~Hz}, 2 \mathrm{H}), 7.39$ (t, $J=7.4 \mathrm{~Hz}$, $1 \mathrm{H}), 7.22(\mathrm{~m}, 2 \mathrm{H}), 7.00(\mathrm{~d}, J=1.0 \mathrm{~Hz}, 1 \mathrm{H}), 2.83(\mathrm{q}, J=7.5 \mathrm{~Hz}, 2 \mathrm{H}), 1.36(\mathrm{t}, J=7.5 \mathrm{~Hz}, 3 \mathrm{H})$; ${ }^{13} \mathrm{C}$ NMR $\left(101 \mathrm{MHz}, \mathrm{CDCl}_{3}\right) \delta 139.5,134.4,129.2,128.4,128.0,127.5,125.7,122.0,120.8$, 119.8, 119.4, 118.4, 18.6, 14.6; IR (Neat): 3440, 2963, 2925, 1634, 1428, 1338, $757 \mathrm{~cm}^{-1}$; HRMS (ESI) $m / z$ calc. for $\mathrm{C}_{16} \mathrm{H}_{15} \mathrm{~N}(\mathrm{M}+\mathrm{H})^{+} 222.1277$, found 222.1281 .

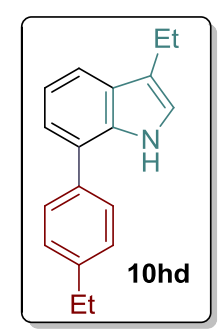

3-Ethyl-7-(4-ethylphenyl)-1H-indole (10hd): Prepared by following General procedure E. Obtained as a white solid $(0.053 \mathrm{~g}$, Yield $=58 \%) ;$ m.p. $=48-50{ }^{0} \mathrm{C} ; \mathrm{R}_{\mathrm{f}}=0.45$ (Ethyl Acetate/Hexane: 10/90); ${ }^{1} \mathrm{H}$ NMR $\left(500 \mathrm{MHz} \mathrm{CDCl}_{3}\right) \delta 8.17(\mathrm{~s}, 1 \mathrm{H}), 7.62-7.59(\mathrm{~m}, 1 \mathrm{H}), 7.58-$ $7.55(\mathrm{~m}, 2 \mathrm{H}), 7.34(\mathrm{~d}, J=8.3 \mathrm{~Hz}, 2 \mathrm{H}), 7.22-7.19(\mathrm{~m}, 2 \mathrm{H}), 7.01-6.98(\mathrm{~m}, 1 \mathrm{H}), 2.83(\mathrm{qd}, J=$ $7.5,0.9 \mathrm{~Hz}, 2 \mathrm{H}), 2.74$ (q, $J=7.6 \mathrm{~Hz}, 2 \mathrm{H}), 1.37(\mathrm{t}, J=7.5 \mathrm{~Hz}, 3 \mathrm{H}), 1.32(\mathrm{t}, J=7.6 \mathrm{~Hz}, 3 \mathrm{H}) ;{ }^{13} \mathrm{C}$ NMR $\left(126 \mathrm{MHz}, \mathrm{CDCl}_{3}\right) \delta 143.6,136.8,134.4,128.7,128.3,127.9,125.7,121.9,120.8,119.7$, 119.3, 118.1, 28.8, 18.6, 15.7, 14.6; IR (Neat): 3440, 2964, 2928, 1513, 1486, 1416, 1091, 795, $745 \mathrm{~cm}^{-1}$; HRMS (ESI) $\mathrm{m} / z$ calc. for $\mathrm{C}_{18} \mathrm{H}_{19} \mathrm{~N}(\mathrm{M}+\mathrm{H})^{+} 250.1590$, found 250.1600 .

\section{Transannulation towards 7-vinylindoles}
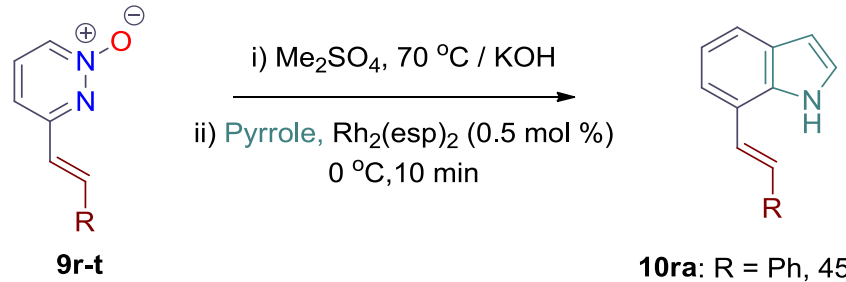

10ra: $\mathrm{R}=\mathrm{Ph}, 45 \%$ 10sa: $\mathrm{R}=\mathrm{C}_{6} \mathrm{H}_{4}(4-\mathrm{Me}), 40 \%$ 10ta: $\mathrm{R}=\mathrm{C}_{6} \mathrm{H}_{4}(4-\mathrm{OMe}), 43 \%$ 


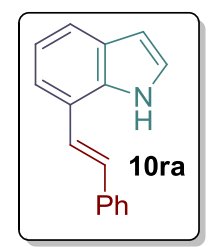

(E)-7-Styryl-1H-indole (10ra): ${ }^{15}$ Prepared by following General procedure E. Obtained as a brown color solid $(0.036 \mathrm{~g}$, Yield $=45 \%)$; m.p. $=152-154{ }^{0} \mathrm{C} ; \mathrm{R}_{\mathrm{f}}=0.5$ (Ethyl Acetate/Hexane: 20/80); ${ }^{1} \mathrm{H} \mathrm{NMR}\left(400 \mathrm{MHz}, \mathrm{CDCl}_{3}\right) \delta 8.46(\mathrm{~s}, 1 \mathrm{H}), 7.60(\mathrm{~d}, J=7.9 \mathrm{~Hz}, 1 \mathrm{H}), 7.56$ (d, J = 7.5 Hz, 2H), $7.42-7.34(\mathrm{~m}, 4 \mathrm{H}), 7.30(\mathrm{~d}, J=7.3 \mathrm{~Hz}, 1 \mathrm{H}), 7.26(\mathrm{dd}, J=6.8,4.0 \mathrm{~Hz}, 1 \mathrm{H}), 7.17(\mathrm{dd}, J=$ 16.7, 9.2 Hz, 2H), $6.61(\mathrm{dd}, J=2.9,2.1 \mathrm{~Hz}, 1 \mathrm{H}) ;{ }^{13} \mathrm{C} \mathrm{NMR}\left(101 \mathrm{MHz}, \mathrm{CDCl}_{3}\right) \delta 137.6,133.9$, 129.9, 128.9, 128.7, 127.9, 126.5, 125.3, 124.4, 121.5, 120.6, 120.6, 120.4, 103.4; IR (Neat): 3410, 2922, 2850, 1616, 1257, 1106, $792 \mathrm{~cm}^{-1}$; HRMS (ESI) $\mathrm{m} / z$ calc. for $\mathrm{C}_{16} \mathrm{H}_{13} \mathrm{~N}(\mathrm{M}+\mathrm{H})^{+}$ 220.1121 , found 220.1132 .

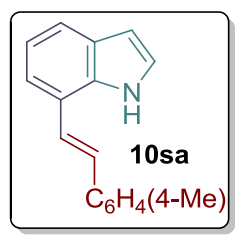

(E)-7-(4-Methylstyryl)-1H-indole (10sa): Prepared by following General procedure E. Obtained as a white solid $(0.021 \mathrm{~g}$, Yield $=40 \%) ; \mathrm{m} . \mathrm{p} .=164-166{ }^{0} \mathrm{C} ; \mathrm{R}_{\mathrm{f}}=0.5$ (Ethyl Acetate/Hexane: 20/80); ${ }^{1} \mathrm{H}$ NMR $\left(400 \mathrm{MHz}, \mathrm{CDCl}_{3}\right) \delta 8.46(\mathrm{~s}, 1 \mathrm{H}), 7.58(\mathrm{~d}, J=7.8 \mathrm{~Hz}, 1 \mathrm{H})$, $7.45(\mathrm{~d}, J=8.0 \mathrm{~Hz}, 2 \mathrm{H}), 7.38-7.28(\mathrm{~m}, 2 \mathrm{H}), 7.27-7.23(\mathrm{~m}, 1 \mathrm{H}), 7.19(\mathrm{~d}, J=7.9 \mathrm{~Hz}, 2 \mathrm{H}), 7.18$ $-7.11(\mathrm{~m}, 2 \mathrm{H}), 6.60(\mathrm{dd}, J=3.0,2.1 \mathrm{~Hz}, 1 \mathrm{H}), 2.38(\mathrm{~s}, 3 \mathrm{H}) ;{ }^{13} \mathrm{C} \mathrm{NMR}\left(101 \mathrm{MHz}, \mathrm{CDCl}_{3}\right) \delta$ $137.8,134.8,133.9,129.9,129.6,128.6,126.5$, 124.3, 124.2, 121.7, 120.5, 120.4, 120.7, 103.4, 21.4; IR (Neat): 3473, 3458, 2912, 2852, 1641, 1628, 1286, 1018, 800, $719 \mathrm{~cm}^{-1}$; HRMS (ESI) $m / z$ calc. for $\mathrm{C}_{17} \mathrm{H}_{15} \mathrm{~N}(\mathrm{M}+\mathrm{H})^{+} 234.1277$, found 234.1265 .

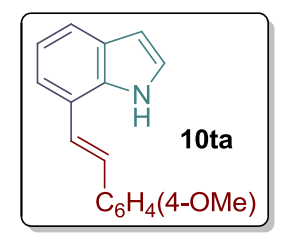

(E)-7-(4-Methoxystyryl)-1H-indole (10ta): Prepared by following General procedure E. Obtained as a brown semi solid (0.022 g, Yield $=43 \%) ; \mathrm{R}_{\mathrm{f}}=0.5$ (Ethyl Acetate/Hexane: $\left.20 / 80\right)$; 
${ }^{1} \mathrm{H}$ NMR $\left(500 \mathrm{MHz}, \mathrm{CDCl}_{3}\right) \delta 8.50(\mathrm{~s}, 1 \mathrm{H}), 7.60(\mathrm{~d}, J=7.6 \mathrm{~Hz}, 1 \mathrm{H}), 7.53(\mathrm{~d}, J=8.8 \mathrm{~Hz}, 2 \mathrm{H})$, $7.37(\mathrm{~d}, J=7.3 \mathrm{~Hz}, 1 \mathrm{H}), 7.33-7.28(\mathrm{~m}, 3 \mathrm{H}), 7.19-7.14(\mathrm{~m}, 2 \mathrm{H}), 6.96(\mathrm{~d}, J=8.7 \mathrm{~Hz}, 2 \mathrm{H}), 6.63$ $(\mathrm{dd}, J=3.1,2.0 \mathrm{~Hz}, 1 \mathrm{H}), 3.88(\mathrm{~s}, 3 \mathrm{H}) ;{ }^{13} \mathrm{C} \mathrm{NMR}\left(176 \mathrm{MHz}, \mathrm{CDCl}_{3}\right) \delta 159.5,133.8,130.4$, $129.5,128.6,127.7,124.3,123.0,121.8,120.4,120.3,120.2,114.3,103.4,55.5$; IR (Neat): 3430, 3388, 2924, 2853, 1615, 1580, 1338, 1246, 1174, 1029, $729 \mathrm{~cm}^{-1}$; HRMS (ESI) m/z calc. for $\mathrm{C}_{17} \mathrm{H}_{15} \mathrm{NO}(\mathrm{M}+\mathrm{H})^{+} 250.1226$, found 250.1207 .

\section{Synthetic applications of transannulation}
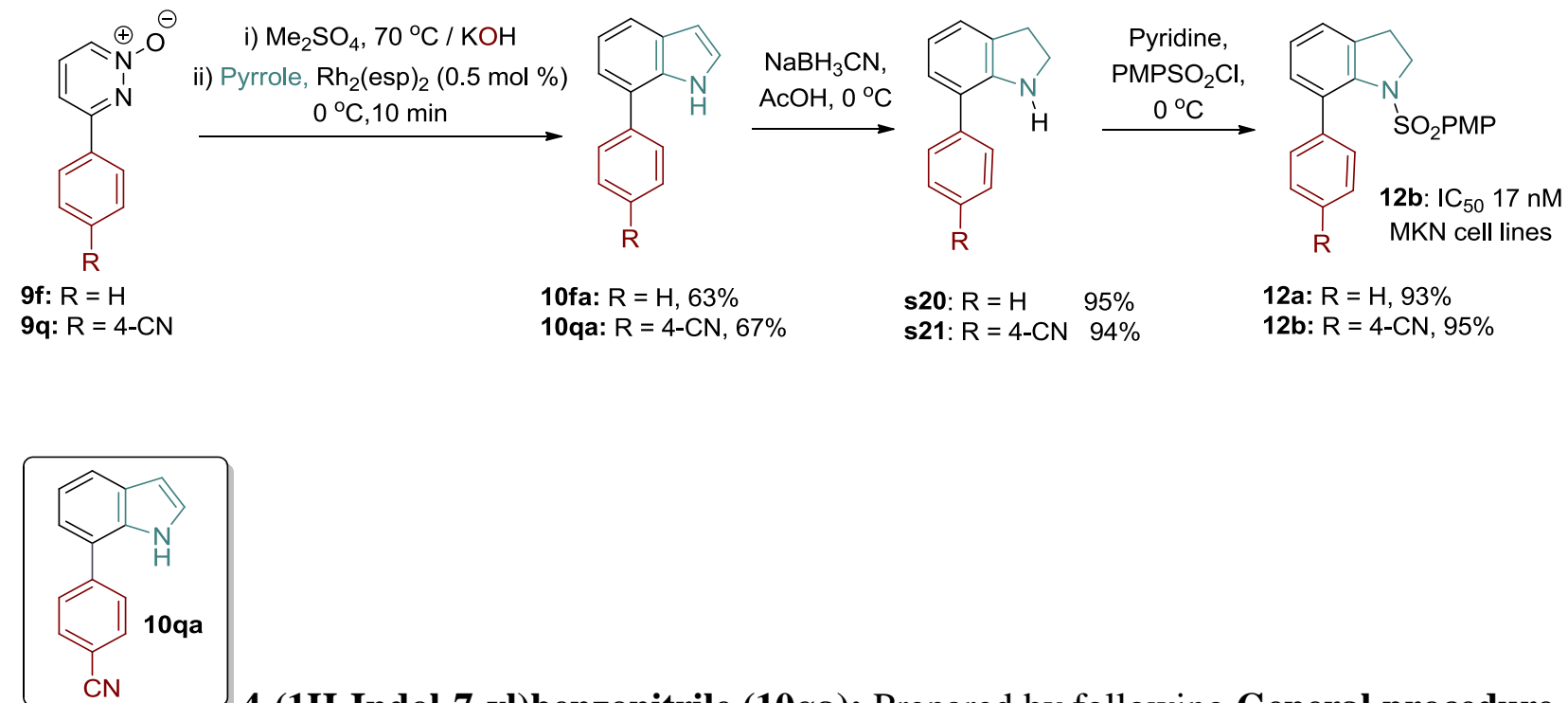

4-(1H-Indol-7-yl)benzonitrile (10qa): Prepared by following General procedure

E. Obtained as a yellow solid (0.054 g, Yield $=67 \%$ ); m.p. $=198-200{ }^{\circ} \mathrm{C} ; \mathrm{R}_{\mathrm{f}}=0.3$ (Ethyl Acetate/Hexane : 10/90); ${ }^{1} \mathrm{H}$ NMR $\left(500 \mathrm{MHz}, \mathrm{CDCl}_{3}\right) \delta 8.44(\mathrm{~s}, 1 \mathrm{H}), 7.83-7.76(\mathrm{~m}, 4 \mathrm{H}), 7.75-$ $7.72(\mathrm{~m}, 1 \mathrm{H}), 7.28(\mathrm{t}, J=2.7 \mathrm{~Hz}, 1 \mathrm{H}), 7.27(\mathrm{~d}, J=7.0 \mathrm{~Hz}, 1 \mathrm{H}), 7.24(\mathrm{~d}, J=1.9 \mathrm{~Hz}, 1 \mathrm{H}), 6.68$ $(\mathrm{dd}, J=3.2,2.0 \mathrm{~Hz}, 1 \mathrm{H}) ;{ }^{13} \mathrm{C} \mathrm{NMR}\left(126 \mathrm{MHz}, \mathrm{CDCl}_{3}\right) \delta 144.3,133.4,133.1,129.0,128.9$, 124.9, 123.7, 122.3, 121.6, 120.6, 119.0, 111.1, 103.6; IR (Neat): 3410, 3356, 2226, 1597, 1480, 1332, 1267, 1203, 842, $729 \mathrm{~cm}^{-1}$; HRMS (ESI) $m / z$ calc. for $\mathrm{C}_{15} \mathrm{H}_{10} \mathrm{~N}_{2}(\mathrm{M}+\mathrm{H})^{+} 219.0917$, found 219.0911 .

\section{Preparation of 7-arylindolines s20 and s21:}

\section{General procedure F:}

A $10 \mathrm{ml}$ round-bottom flask was charged with 7-arylindole $\mathbf{1 0 f a}$ or $\mathbf{1 0 q a}(0.12 \mathrm{mmol}, 1$ equiv) and $1 \mathrm{ml}$ acetic acid and cooled to $0{ }^{\circ} \mathrm{C}$. To the contents sodium cyanoborohydride $(0.32 \mathrm{mmol}$, 
2.5 equiv) was added portion wise. After $3 \mathrm{~h}$, the reaction mixture was quenched with aq $\mathrm{NaHCO}_{3}$ and extracted with ethyl acetate. The organic layer was dried over anhydrous Sodium sulfate and concentrated under reduced pressure. The resulting crude indoline product was purified by a flash column chromatography by using ethyl acetate and petroleum ether as eluent.

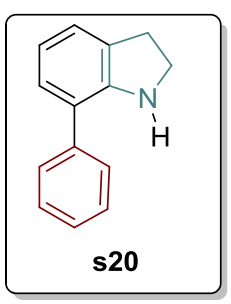

7-Phenylindoline (s20): Prepared by following procedure F. Obtained as a colorless liquid $(0.022 \mathrm{~g}$, Yield $=95 \%) ; \mathrm{R}_{\mathrm{f}}=0.45$ (Ethyl Acetate/Hexane: $\left.10 / 90\right) ;{ }^{1} \mathrm{H}$ NMR $\left(500 \mathrm{MHz}, \mathrm{CDCl}_{3}\right) \delta$ $7.59-7.54(\mathrm{~m}, 2 \mathrm{H}), 7.47-7.42(\mathrm{~m}, 2 \mathrm{H}), 7.35-7.29(\mathrm{~m}, 1 \mathrm{H}), 7.16-7.09(\mathrm{~m}, 2 \mathrm{H}), 6.81(\mathrm{t}, J=$ $7.5 \mathrm{~Hz}, 1 \mathrm{H}), 4.08(\mathrm{~s}, 1 \mathrm{H}), 3.55(\mathrm{t}, J=8.4 \mathrm{~Hz}, 2 \mathrm{H}), 3.12(\mathrm{t}, J=8.4 \mathrm{~Hz}, 2 \mathrm{H}) ;{ }^{13} \mathrm{C}$ NMR $(126 \mathrm{MHz}$, $\left.\mathrm{CDCl}_{3}\right) \delta 149.2,139.7,129.8,128.8,128.1,127.5,126.9,123.9,123.1,119.1,47.5,30.2$; IR (Neat): 3430, 3388, 2924, 2853, 1578, 1520, 1246, 1174, 1029, $729 \mathrm{~cm}^{-1}$; HRMS (ESI) $\mathrm{m} / z$ calc. for $\mathrm{C}_{14} \mathrm{H}_{13} \mathrm{~N}(\mathrm{M}+\mathrm{H})^{+}$196.1121, found 196.1147 .

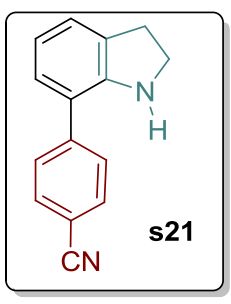

4-(Indolin-7-yl)benzonitrile (s21): Prepared by following procedure F. Obtained as a yellow solid $(0.024 \mathrm{~g}$, Yield $=94 \%) ;$ m.p. $=146-148{ }^{\circ} \mathrm{C} ; \mathrm{R}_{\mathrm{f}}=0.4\left(\right.$ Ethyl Acetate/Hexane : 20/80); ${ }^{1} \mathrm{H}$ $\operatorname{NMR}\left(500 \mathrm{MHz}, \mathrm{CDCl}_{3}\right) \delta 7.72-7.65(\mathrm{~m}, 4 \mathrm{H}), 7.16(\mathrm{dd}, J=7.2,1.1 \mathrm{~Hz}, 1 \mathrm{H}), 7.07(\mathrm{~d}, J=7.7$ $\mathrm{Hz}, 1 \mathrm{H}), 6.81(\mathrm{t}, J=7.5 \mathrm{~Hz}, 1 \mathrm{H}), 4.03(\mathrm{~s}, 1 \mathrm{H}), 3.57(\mathrm{t}, J=8.4 \mathrm{~Hz}, 2 \mathrm{H}), 3.11(\mathrm{t}, J=8.4 \mathrm{~Hz}, 2 \mathrm{H})$; ${ }^{13} \mathrm{C}$ NMR $\left(126 \mathrm{MHz}, \mathrm{CDCl}_{3}\right) \delta 149.3,144.6,132.7,130.5,128.6,127.3,125.1,120.9,119.4$, 119.1, 110.0, 47.4, 30.0; IR (Neat): 3336, 2941, 2843, 2225, 1587, 1247, 1022, $750 \mathrm{~cm}^{-1}$; HRMS (ESI) $m / z$ calc. for $\mathrm{C}_{15} \mathrm{H}_{12} \mathrm{~N}_{2}(\mathrm{M}+\mathrm{H})^{+} 221.1073$, found 221.1098 .

N-protection of indolines: Formation of 12a and 12b 


\section{General Procedure G:}

The reaction was carried out according to the literature procedure. ${ }^{16} 7$-Aryl indoline $\mathbf{s 2 0}$ or $\mathbf{5 2 1}$ $(0.125 \mathrm{mmol})$ was dissolved in pyridine $(1 \mathrm{ml})$ and 4-methoxyphenylsulfonyl chloride $(0.25$ mmol) was added. The reaction mixture was refluxed for $15 \mathrm{~h}$. The solvent was evaporated under reduced pressure and the residue was purified by a silica gel flash column chromatography to afford 12a or 12b.

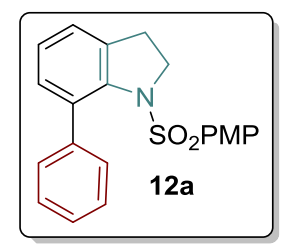

1-((4-Methoxyphenyl)sulfonyl)-7-phenylindoline (12a): ${ }^{16}$ Prepared by following procedure G. Obtained as a white solid (0.042 g, Yield $=93 \%) ; R_{\mathrm{f}}=0.3$ (Ethyl Acetate/Hexane: 20/80); The desired product data was matched with known literature compound; ${ }^{1} \mathrm{H}$ NMR (500 MHz, $\left.\mathrm{CDCl}_{3}\right) \delta 7.41-7.38(\mathrm{~m}, 2 \mathrm{H}), 7.16-7.11(\mathrm{~m}, 2 \mathrm{H}), 7.06-7.01(\mathrm{~m}, 4 \mathrm{H}), 6.93(\mathrm{t}, J=7.5 \mathrm{~Hz}, 1 \mathrm{H})$, $6.75(\mathrm{dd}, J=7.4,1.2 \mathrm{~Hz}, 1 \mathrm{H}), 6.51-6.47(\mathrm{~m}, 2 \mathrm{H}), 3.74(\mathrm{t}, J=7.4 \mathrm{~Hz}, 2 \mathrm{H}), 3.52(\mathrm{~s}, 3 \mathrm{H}), 2.05$ (t, $J=7.4 \mathrm{~Hz}, 2 \mathrm{H}) ;{ }^{13} \mathrm{C} \mathrm{NMR}\left(126 \mathrm{MHz}, \mathrm{CDCl}_{3}\right) \delta 163.3,140.7,140.3,138.4,135.9,129.8,129.7$, 129.5, 128.4, 128.4, 127.3, 127.1, 123.7, 113.9, 55.7, 52.2, 29.3.

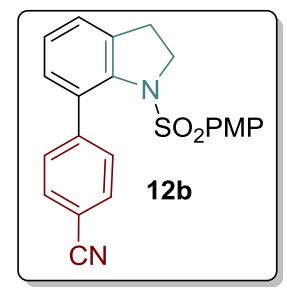

4-(1-((4-Methoxyphenyl)sulfonyl)indolin-7-yl)benzonitrile (12b): ${ }^{16}$ Prepared by following procedure G. Obtained as a white solid $(0.046 \mathrm{~g}$, Yield $=95 \%) ; \mathrm{R}_{\mathrm{f}}=0.3$ (Ethyl Acetate/Hexane: 20/80); The desired product data was matched with known literature compound; ${ }^{1} \mathrm{H}$ NMR (500 $\left.\mathrm{MHz}, \mathrm{CDCl}_{3}\right) \delta 7.80(\mathrm{~d}, J=8.1 \mathrm{~Hz}, 2 \mathrm{H}), 7.72(\mathrm{~d}, J=8.1 \mathrm{~Hz}, 2 \mathrm{H}), 7.34-7.27(\mathrm{~m}, 4 \mathrm{H}), 7.13(\mathrm{~d}, J$ $=6.8 \mathrm{~Hz}, 1 \mathrm{H}), 6.81(\mathrm{~d}, J=8.7 \mathrm{~Hz}, 2 \mathrm{H}), 4.05(\mathrm{t}, J=7.4 \mathrm{~Hz}, 2 \mathrm{H}), 3.84(\mathrm{~s}, 3 \mathrm{H}), 2.34(\mathrm{t}, J=7.3 \mathrm{~Hz}$, $2 \mathrm{H}) ;{ }^{13} \mathrm{C}$ NMR $\left(126 \mathrm{MHz}, \mathrm{CDCl}_{3}\right) \delta 163.5,145.5,140.3,138.8,133.8,132.3,129.7,129.4$, 129.1, 128.9, 127.6, 124.9, 119.4, 114.1, 110.6, 55.7, 52.1, 29.1. 


\section{Characterization of the intermediate 15b:}

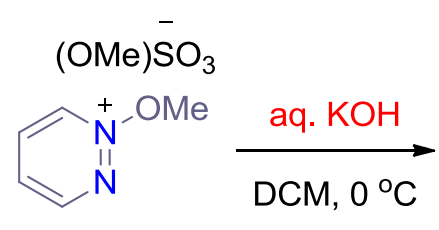

$6 a$

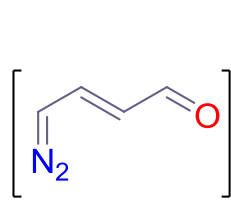

$7 a$

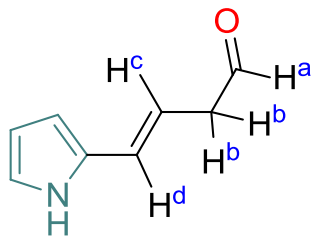

$15 b$

(E)-4-(1H-Pyrrol-2-yl)but-3-enal 15b: To a suspension of 1-methoxypyridazin-1-ium methyl sulfate 6a $(1 \mathrm{mmol})$ in DCM $(10 \mathrm{ml})$ at $0{ }^{\circ} \mathrm{C}$ was added cold $1 \mathrm{~N}$ aq $\mathrm{KOH}(2 \mathrm{ml})$. The organic layer was collected and concentrated to $2 \mathrm{ml}$ volume under reduced pressure at $0{ }^{\circ} \mathrm{C}$. The crude $(E)$-enaldiazomethane 7a was immediately added dropwise over $5 \mathrm{~min}$ to a DCM (2 ml) solution of pyrrole $(0.5 \mathrm{mmol})$ and $\mathrm{Rh}_{2}(\mathrm{esp})_{2}(0.0015 \mathrm{mmol})$ at $-5{ }^{\circ} \mathrm{C}$. The recation mixture was quickly filtered through a celite pad and the solvent was evaporated. NMR analysis indicated the presence of the intermediate $\mathbf{1 5 b}$ as the major product. ${ }^{1} \mathrm{H} \mathrm{NMR}\left(500 \mathrm{MHz}, \mathrm{CDCl}_{3}\right) \delta 9.72(\mathrm{t}, J=$ $\left.1.8 \mathrm{~Hz}, 1 \mathrm{H}^{\mathrm{a}}\right), 8.25(\mathrm{~s}, 1 \mathrm{H}), 6.76(\mathrm{dd}, J=4.0,2.6 \mathrm{~Hz}, 1 \mathrm{H}), 6.40\left(\mathrm{~d}, J=16.2 \mathrm{~Hz}, 1 \mathrm{H}^{\mathrm{d}}\right), 6.23-6.17$ $(\mathrm{m},, 2 \mathrm{H}), 5.81\left(\mathrm{dt}, J=16.1,7.1 \mathrm{~Hz}, 1 \mathrm{H}^{\mathrm{c}}\right), 3.30\left(\mathrm{dt}, J=7.1,1.6 \mathrm{~Hz}, 2 \mathrm{H}^{\mathrm{b}}\right) ;{ }^{13} \mathrm{C} \mathrm{NMR}(126 \mathrm{MHz}$, $\left.\mathrm{CDCl}_{3}\right) \delta 199.7,125.8,119.0,113.5,111.6,109.8,108.7,47.5$.
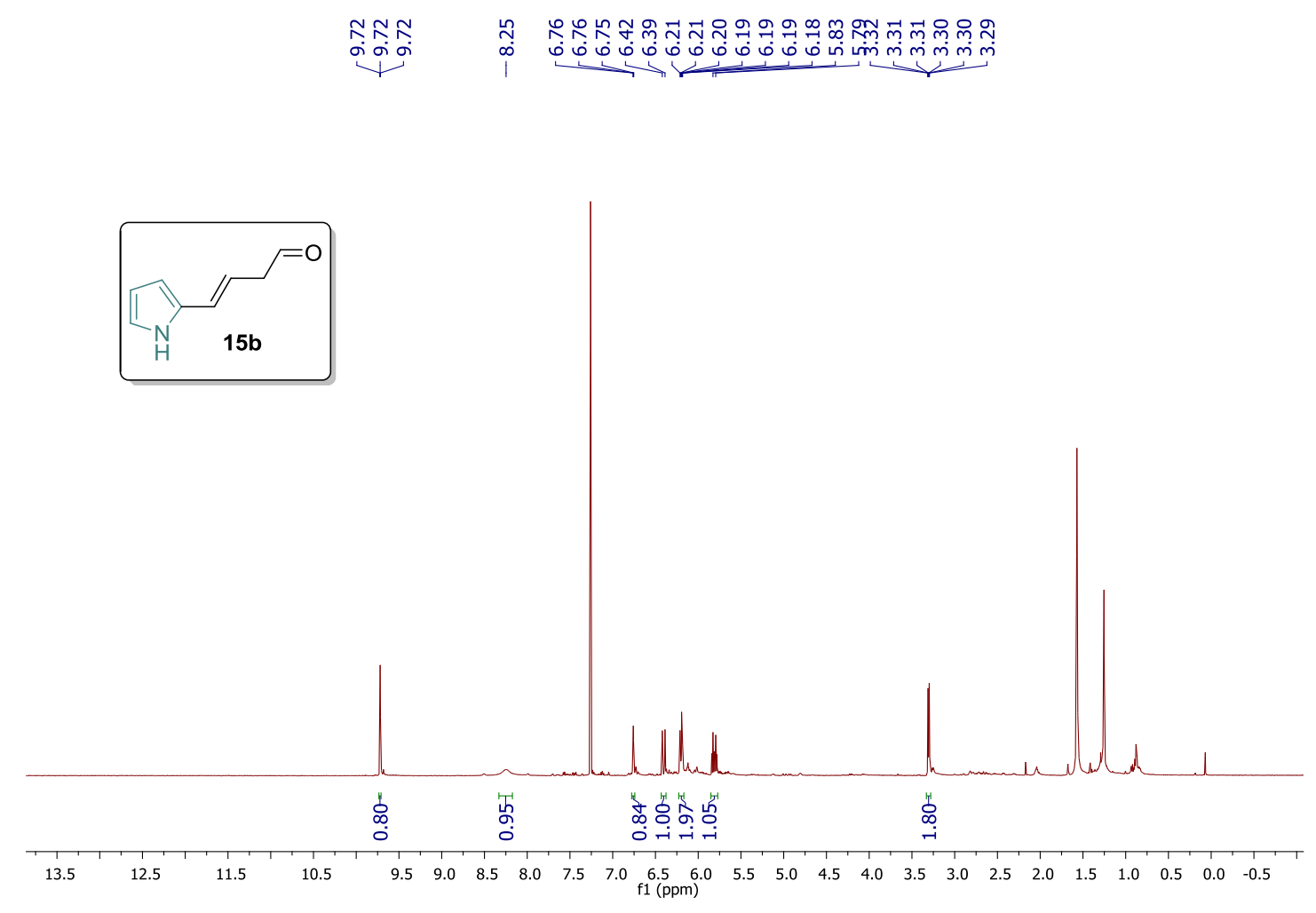

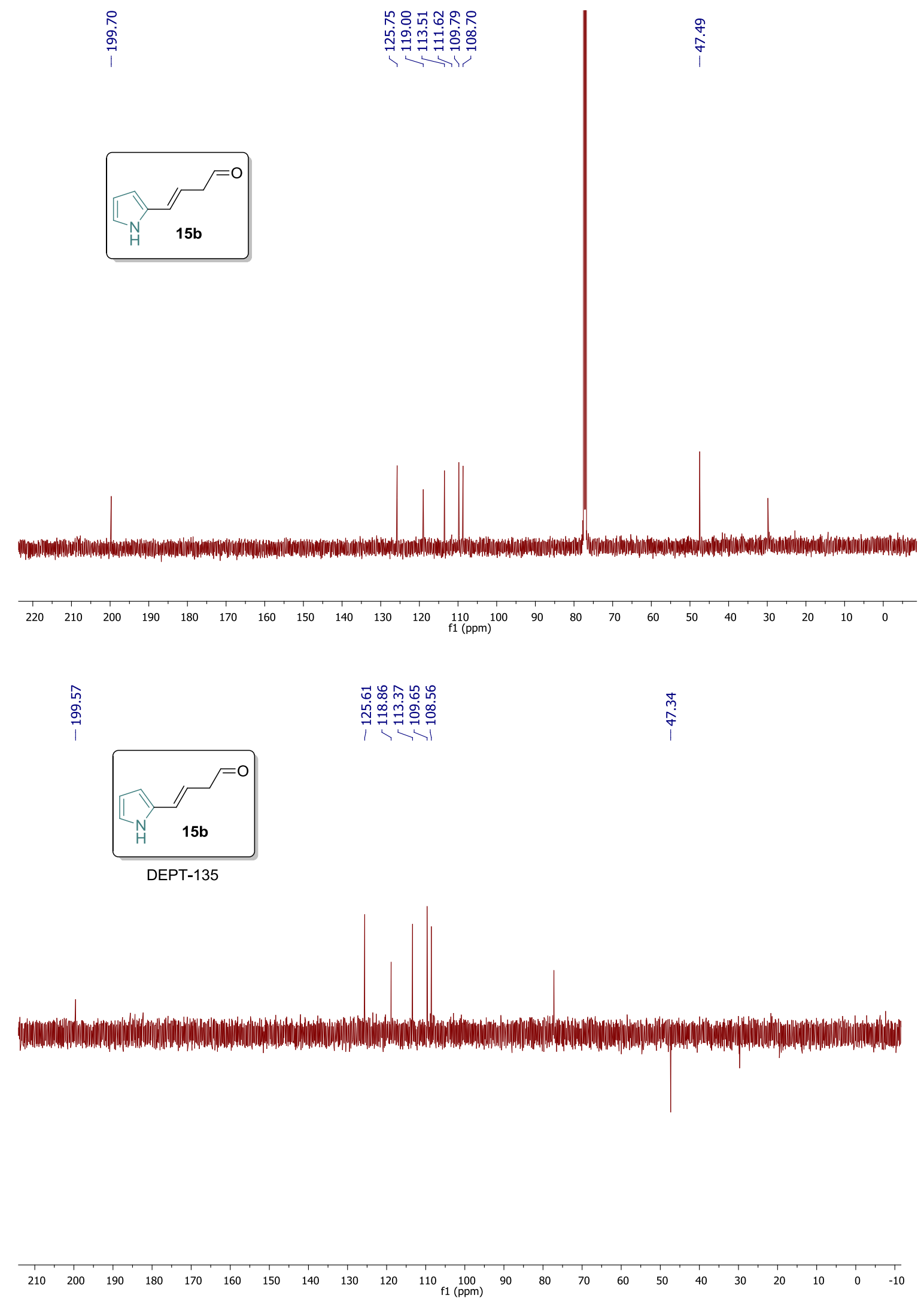


\section{XRD data of 10fc:}
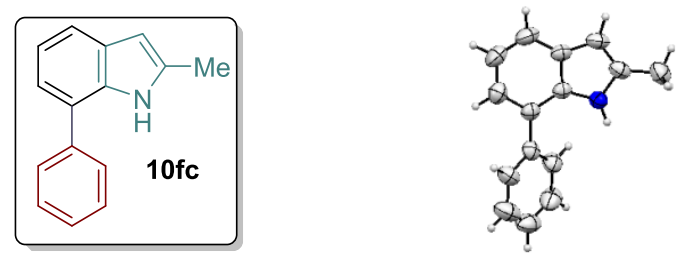

Color code: Carbon (light-gray), Nitrogen (blue), Hydrogen (white).

\begin{tabular}{|l|l|}
\hline CCDC & 1420660 \\
\hline Formula & C15 H12 N \\
\hline Formula Weight & 206.26 \\
\hline Wavelength & 0.71073 \\
\hline Temperature(K) & $298(2)$ \\
\hline Crystal System & Orthorhambic \\
\hline Space Group & $P 212121$ \\
\hline a $(\AA)$ & $6.8195(12)$ \\
\hline b( $(\AA)$ & $8.3525(14)$ \\
\hline c( $(\AA)$ & $20.053(3)$ \\
\hline$\alpha\left(^{\circ}\right)$ & 90 \\
\hline$\beta\left(^{\circ}\right)$ & 90 \\
\hline$\gamma\left(^{\circ}\right)$ & 90 \\
\hline V(cm $\left.{ }^{3}\right)$ & 1142.22 \\
\hline Z & 4 \\
\hline Density $\left(\mathrm{g} \mathrm{cm}^{-3}\right)$ & 1.199 \\
\hline$\mu\left(\mathrm{mm}^{-1}\right)$ & 0.070 \\
\hline F $(000)$ & 436.0 \\
\hline No. of ref. & 1877 \\
\hline Unique ref. & 1477 \\
\hline R1 & 0.048 \\
\hline wR2 & 0.162 \\
\hline G.O.F & 0.997 \\
\hline & \\
\hline
\end{tabular}




\section{References:}

1) Dostal, W.; Heinisch, G.; Lotsch, G. Monatshefte ffir Chemie 1988, 119, 751-759.

2) Wermuth, C. G.; Bourguignon, J. J.; Schlewer, G.; Gies, J. P.; Schoenfelder, A.; Melikian, A.; Bouchet, M. J.; Chantreux, D.; Molimard, J. C.; Heaulme, M.; Chambon, J. P.; Biziere, K. J. Med. Chem. 1987, 30, $239-249$.

3) Tsukamoto, I.; Koshio, H.; Kuramochi, T.; Saitoh, C.; Yanai-Inamura, H.; Nozawa, C. K.; Yamamoto, E.; Yatsu, T.; himada, Y.; Sakamoto, S.; Tsukamoto, S. Bioorg. Med. Chem. 2009, 17, 3130-3141.

4) South, M. S.; Jakuboski, T. L.; Westmeyer, M. D.; Dukesherer, D. R. J. Org. Chem. 1996, 61, 8921-8934.

5) Seiple, I. B.; Su, S.; Rodriguez, R. A.; Gianatassio, R.; Fujiwara, Y.; Sobel, A. L.; Baran, P. S. J. Am. Chem. Soc. 2010, 132, 13194-13196.

6) Swamy, K. M. K.; Park, M. S.; Han, S. J.; Kim, S. K.; Kim, J. H.; Lee, C.; Bang, H.; Kim, Y.; Kim, S. -J.; Yoon, J. Tetrahedron 2005, 61, 10227-10234.

7) Leclerc, J. P.; Fagnou, K. Angew. Chem. Int. Ed. 2006, 45, 7781 - 7786.

8) a) He, Y.; Lin, M.; Li, Z.; Liang, X.; Li, G.; Antilla, J. C. Org. Lett. 2011, 13, 4490-4493. b) Dawande, S. G.; Kanchupalli, V.; Kalepu, J.; Chennamsetti, H.; Lad, B. S.; Katukojvala, S. Angew. Chem. Int. Ed. 2014, 53, 4076-4080.

9) Tsuchiya, T.; Kaneko, C.; Igeta, H. J.C.S. Chem. commun. 1975, 528-529.

10) Su, Y., M.; Hou, Y.; Yin, F.; Xu, Y. M.; Li, Y.; Zheng, X.; Wang, X. S. Org. Lett. 2014, 16, 2958-2961

11) Wendlandt, A. E.; Stahl, S. S. J. Am. Chem. Soc. 2014, 136, 506-512.

12) Cho, C. S.; Kim, J. H.; Kim, T. J.; Shim, S. C. Tetrahydron 2001, 57, 3321 - 3329.

13) Damodara, D.; Arundhathi, R.; Likhar, P. R. Adv. Synth. Catal. 2014, 356, 189 -198.

14) Robbins, D. W.; Boebel, T. A.; Hartwig, J. F. J. Am. Chem. Soc. 2010, 132, 4068-4069.

15) McLaughlin, M. G.; McAdam, C. A.; Cook, M. J. Org. Lett. 2015, 17, 10-13.

16) Chang, J.Y.; Lai, M. J.; Chang, Y. T.; Lee, H.Y.; Cheng, Y. C.; Kuo, C. C.; Su, M. C.; Chang, C. Y.; Liou, J. P. Med. Chem. Commun. 2010, 1, 152-155. 
11. NMR spectra:

는

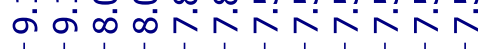
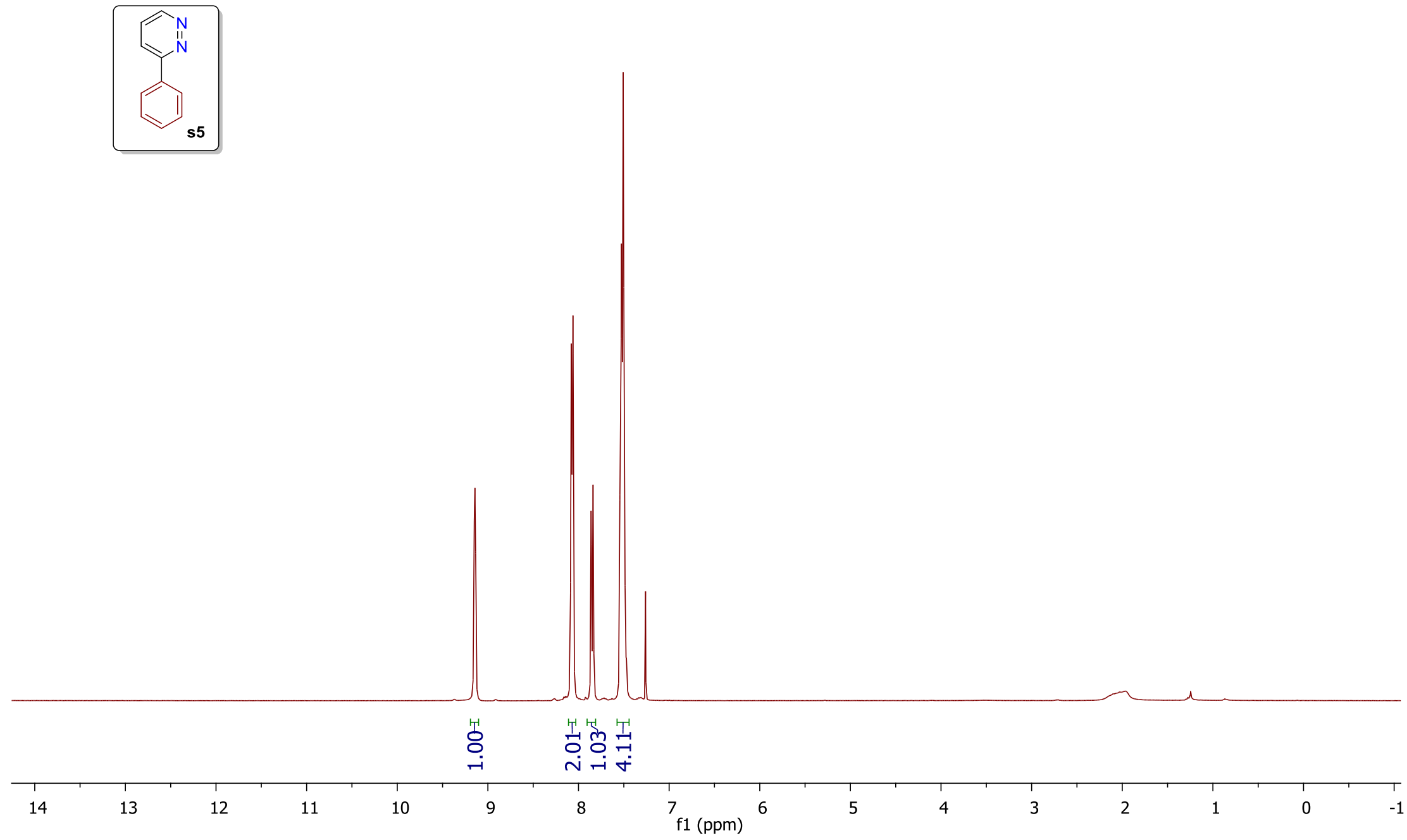


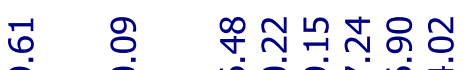

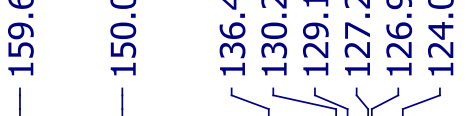
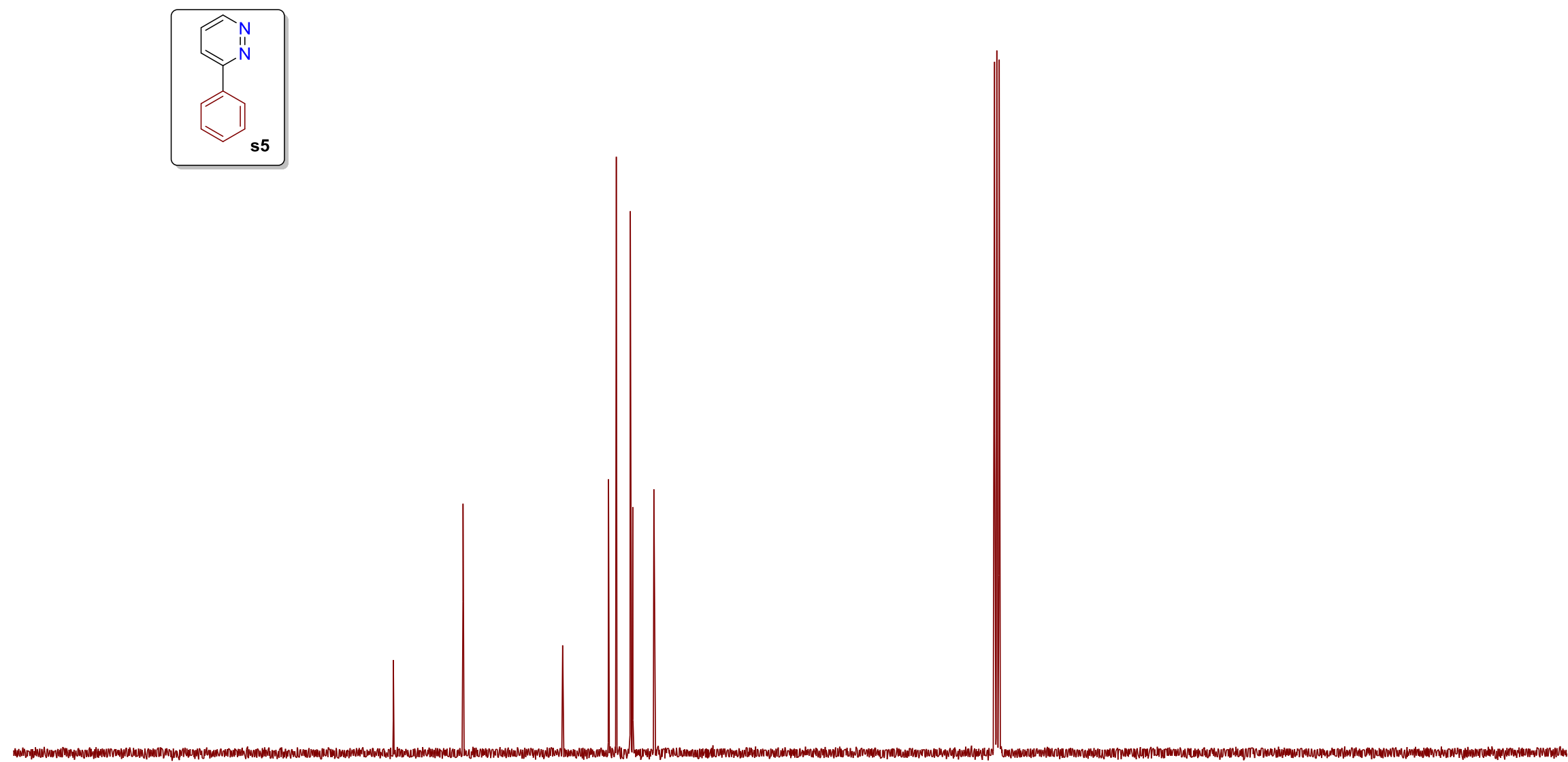


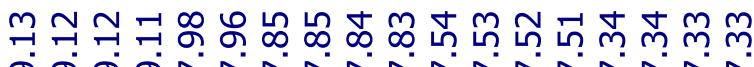

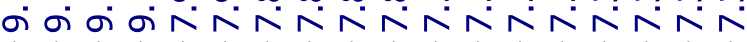

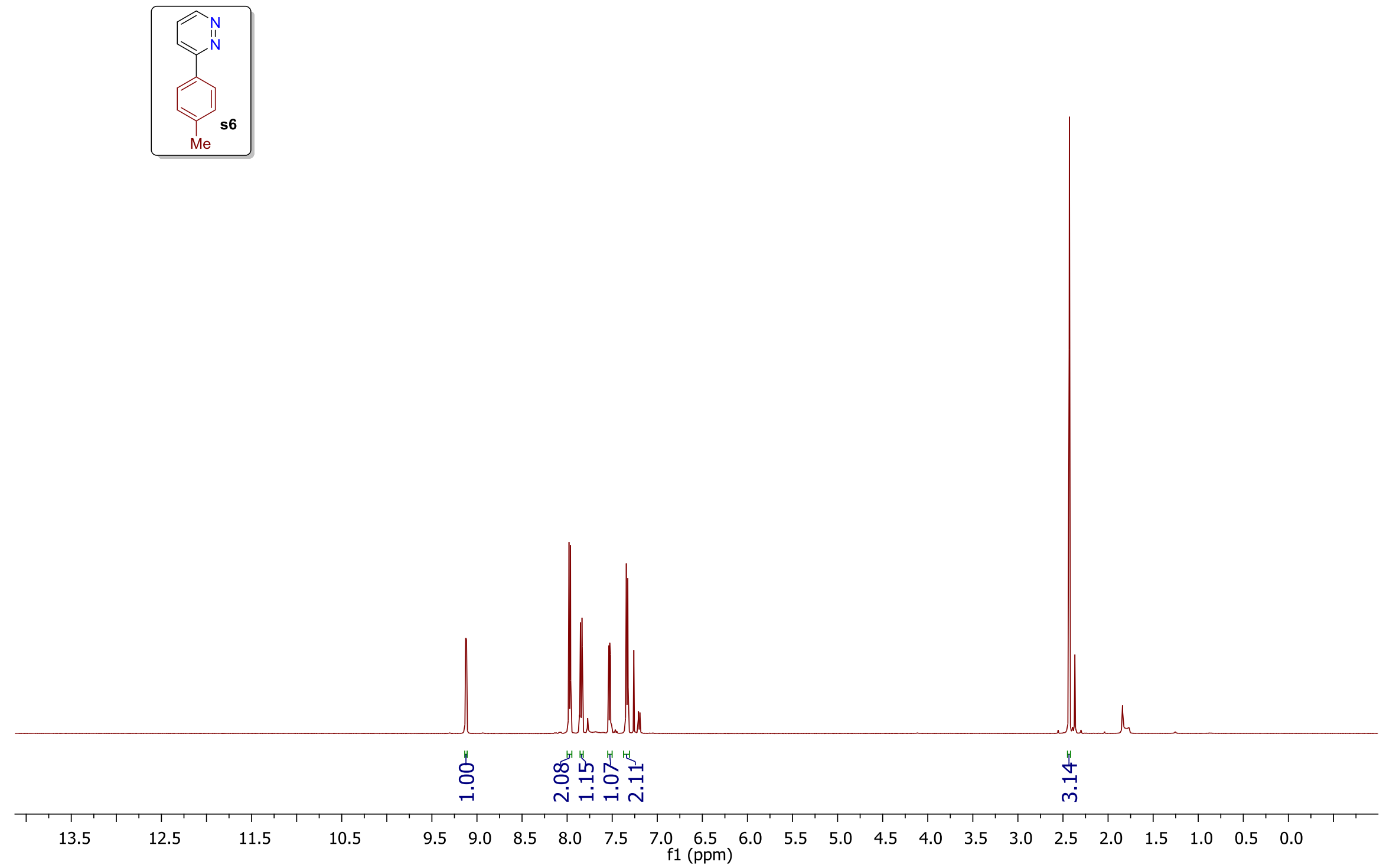

$\rightarrow$ 


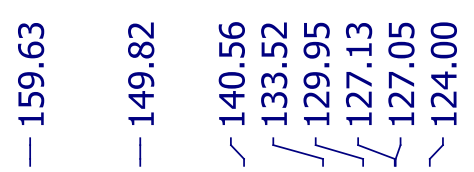
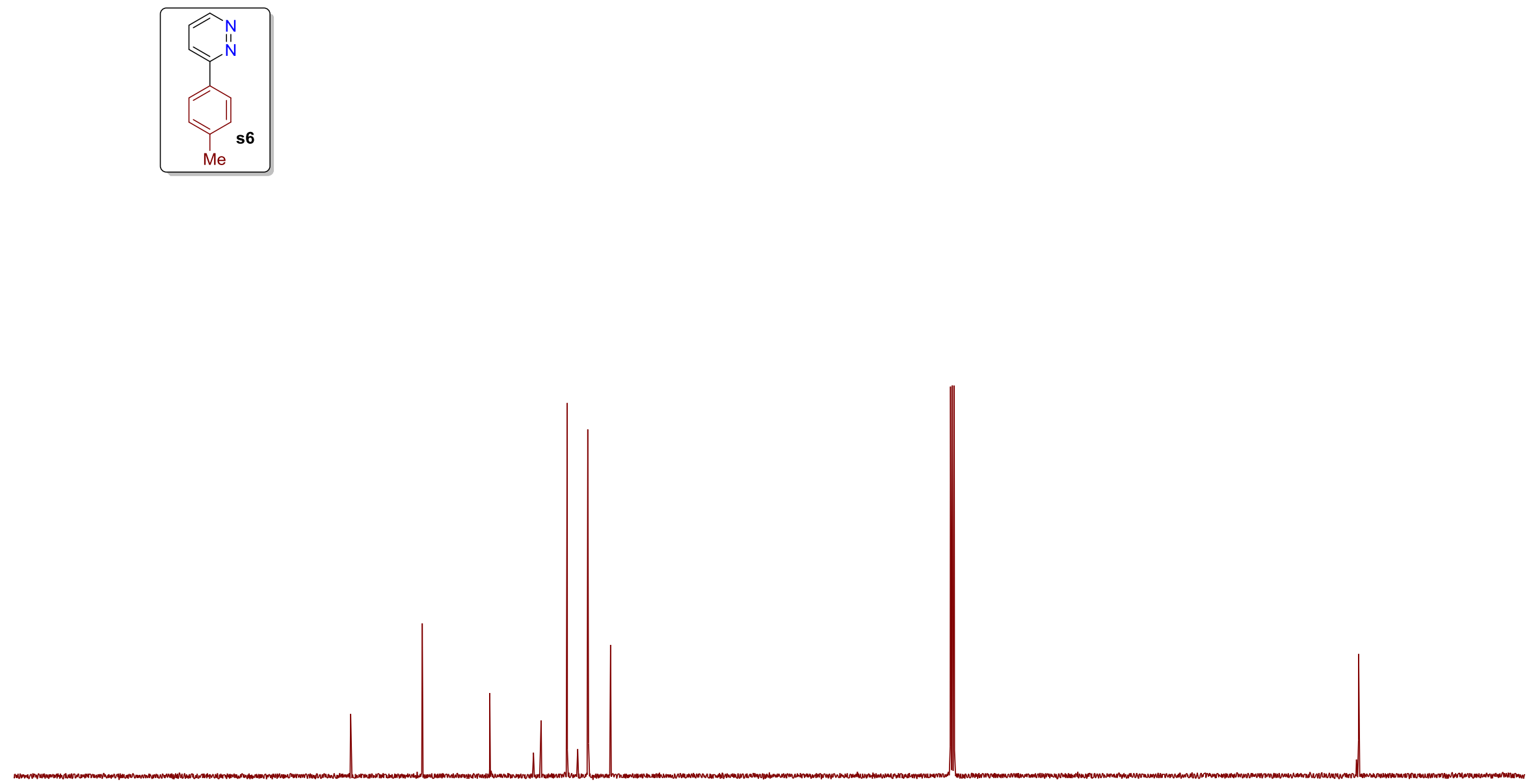

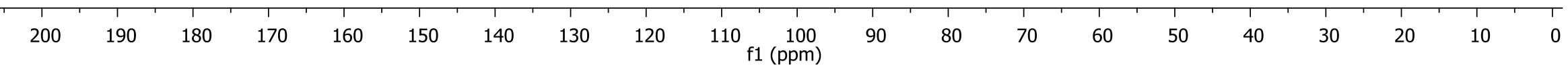




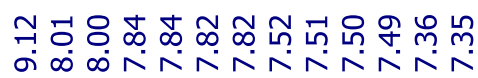

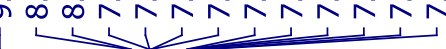

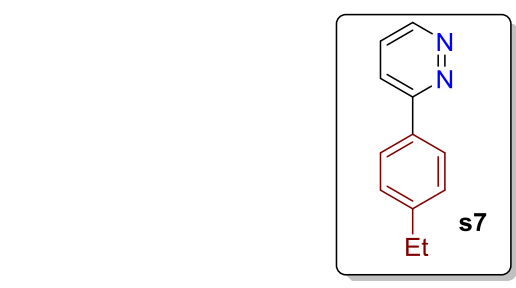

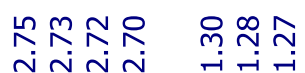

N

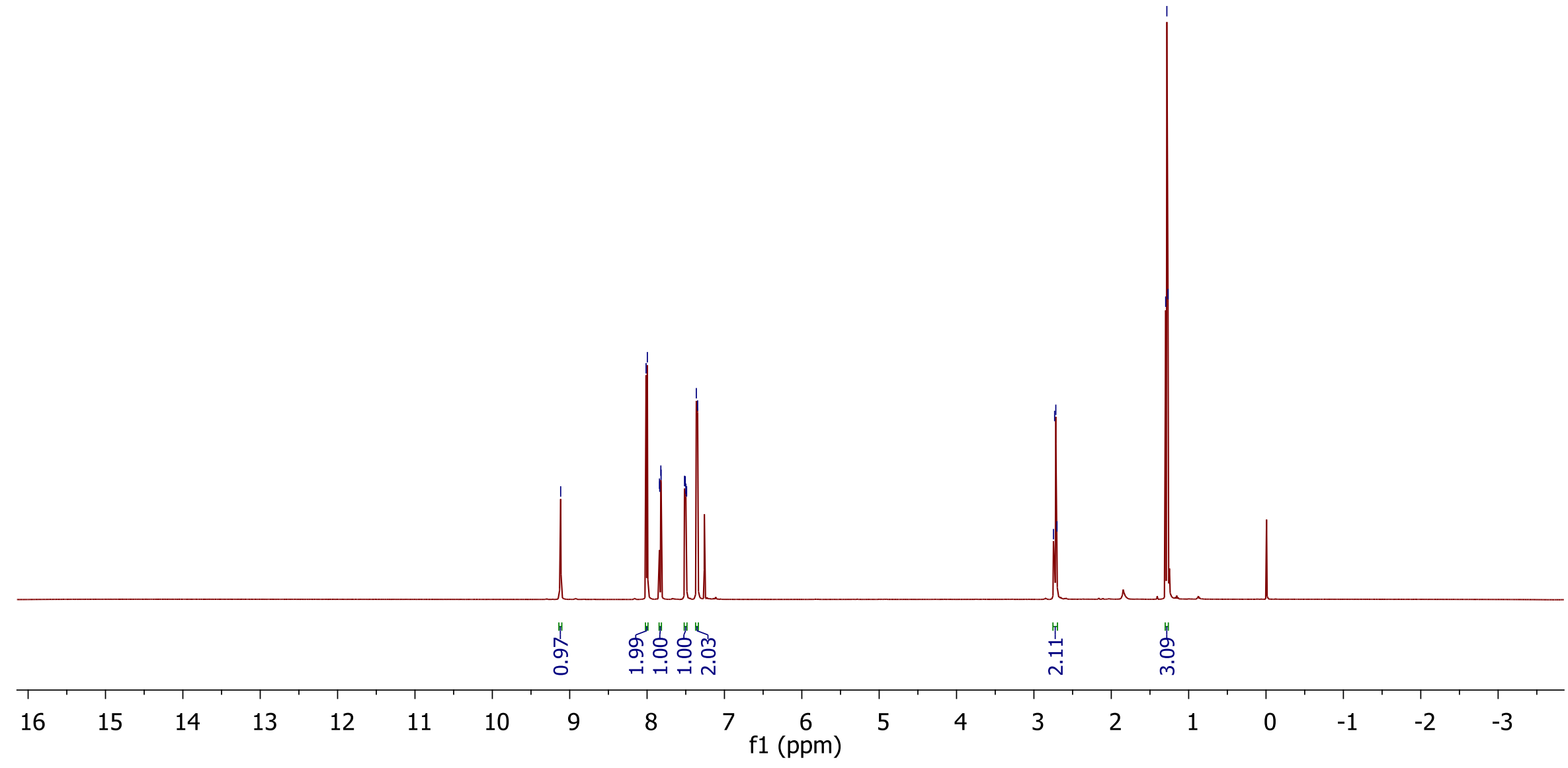



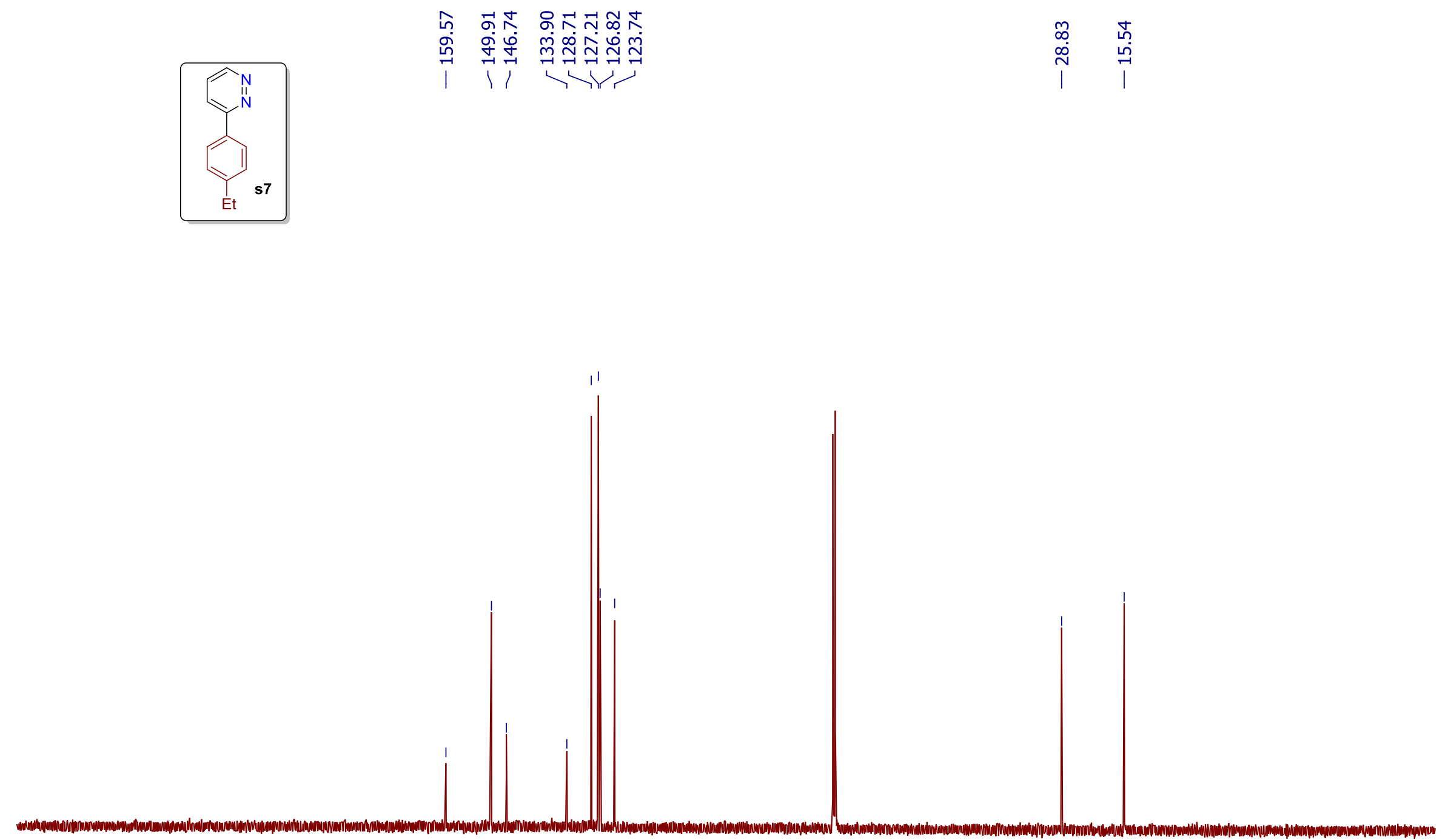

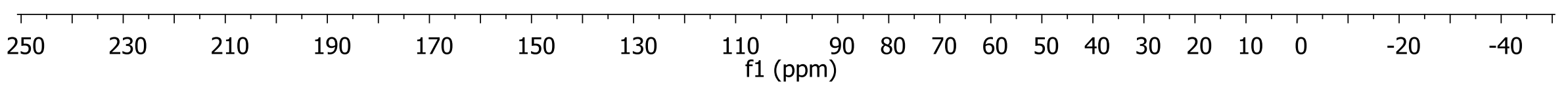


끔 끔 국 궁

बंの

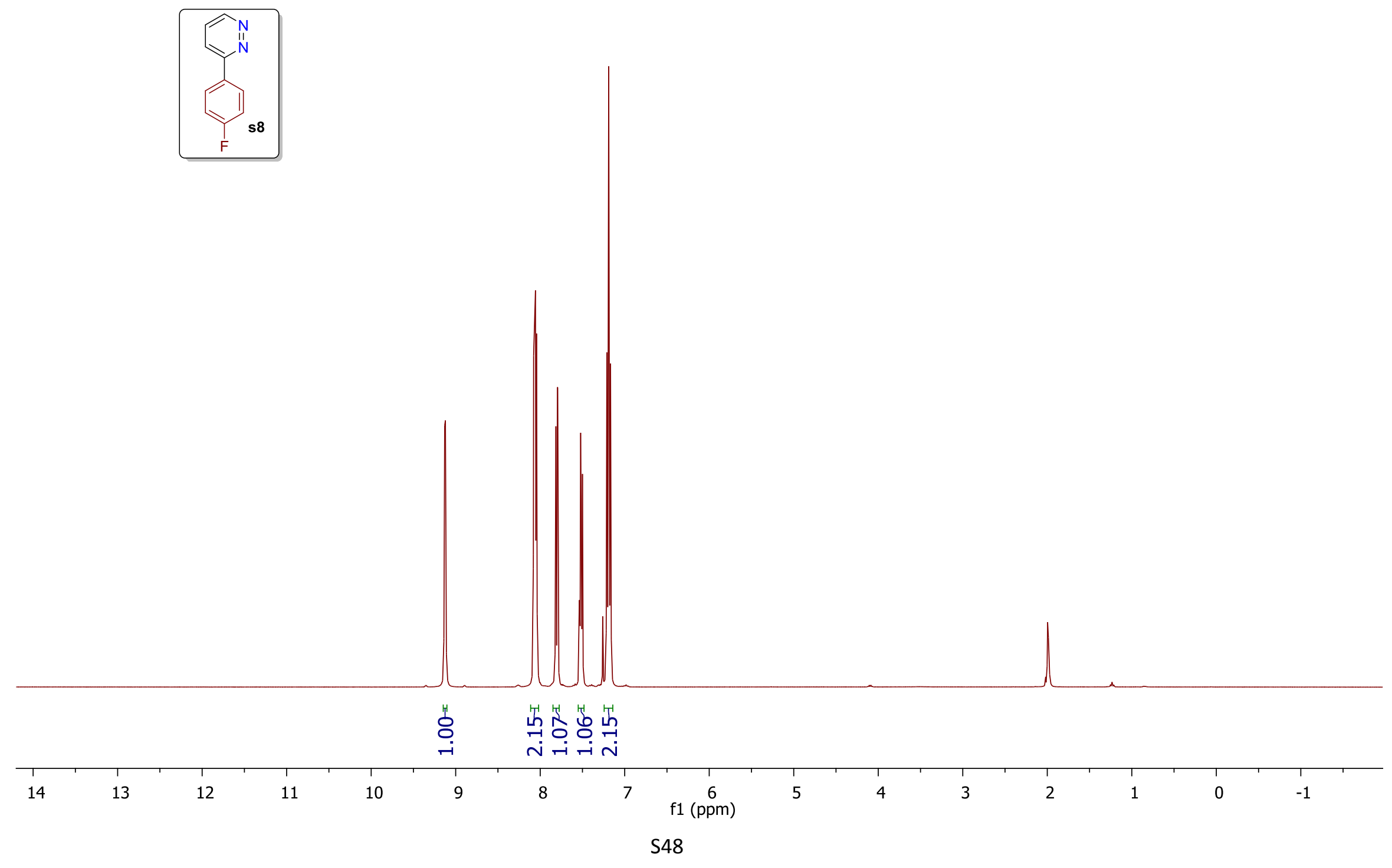

i 


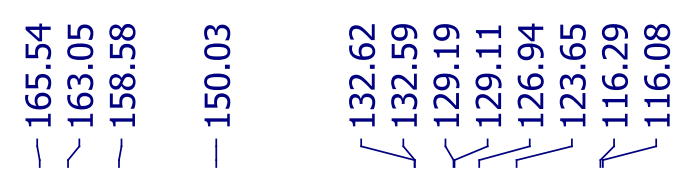
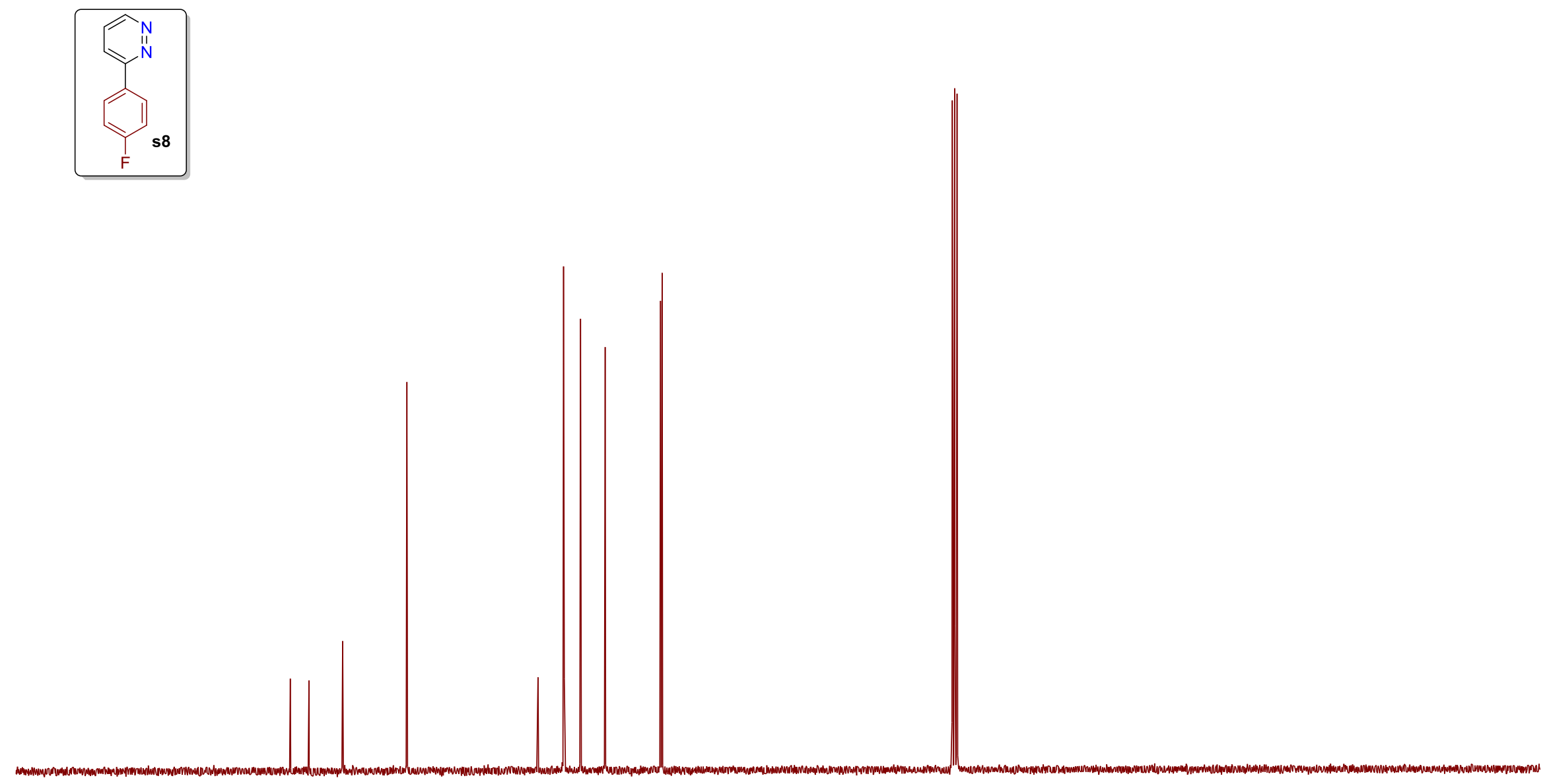

\begin{tabular}{|c|c|c|c|c|c|c|c|c|c|c|c|c|c|c|c|c|c|c|c|}
\hline \multirow{2}{*}{200} & 190 & 1 & ${ }_{170}^{1}$ & & & & & & \multirow{2}{*}{$\frac{1}{110}$} & I & 1 & \multirow{2}{*}{80} & \multirow{2}{*}{70} & \multirow{2}{*}{60} & \multirow{2}{*}{50} & \multirow{2}{*}{10} & \multirow{2}{*}{30} & \multirow{2}{*}{1} & 1 \\
\hline & 190 & 180 & 170 & 160 & 150 & 140 & 130 & 120 & & $\begin{array}{c}100 \\
\mathrm{f} 1(\mathrm{ppm})\end{array}$ & 90 & & & & & & & & 10 \\
\hline
\end{tabular}




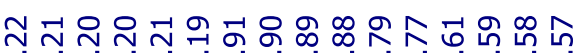

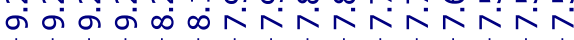
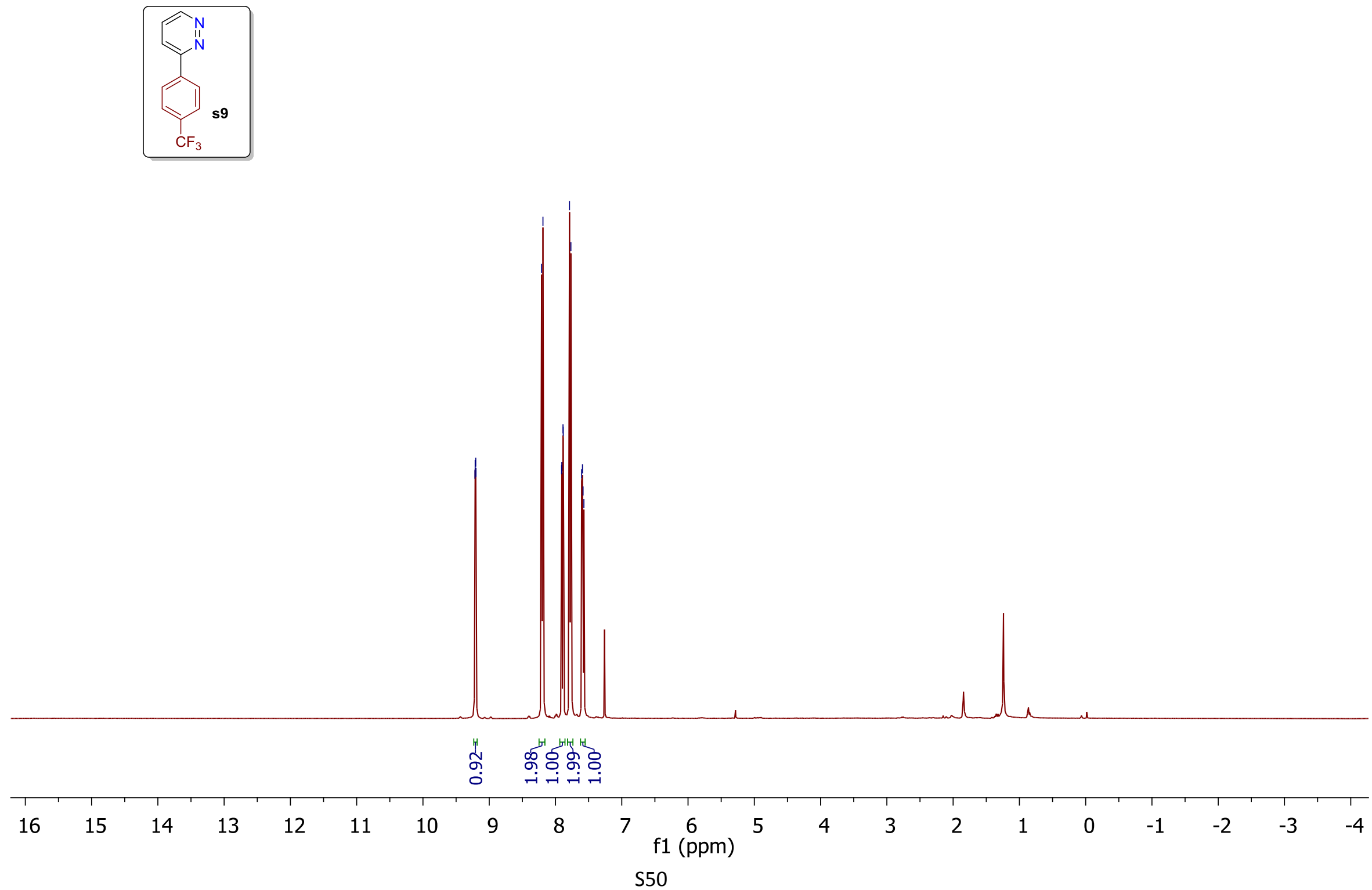

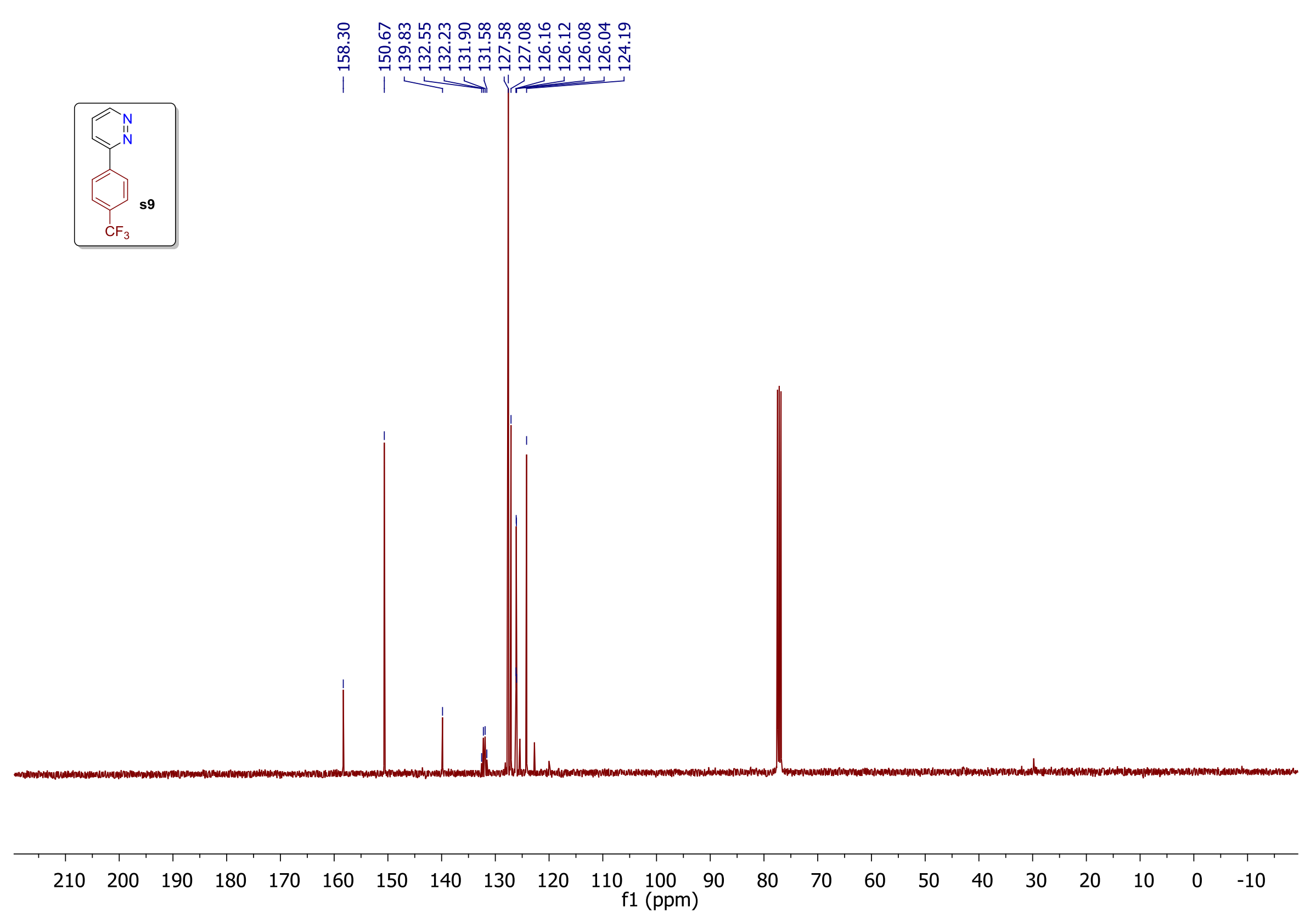


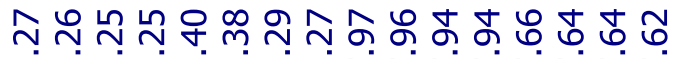

जं
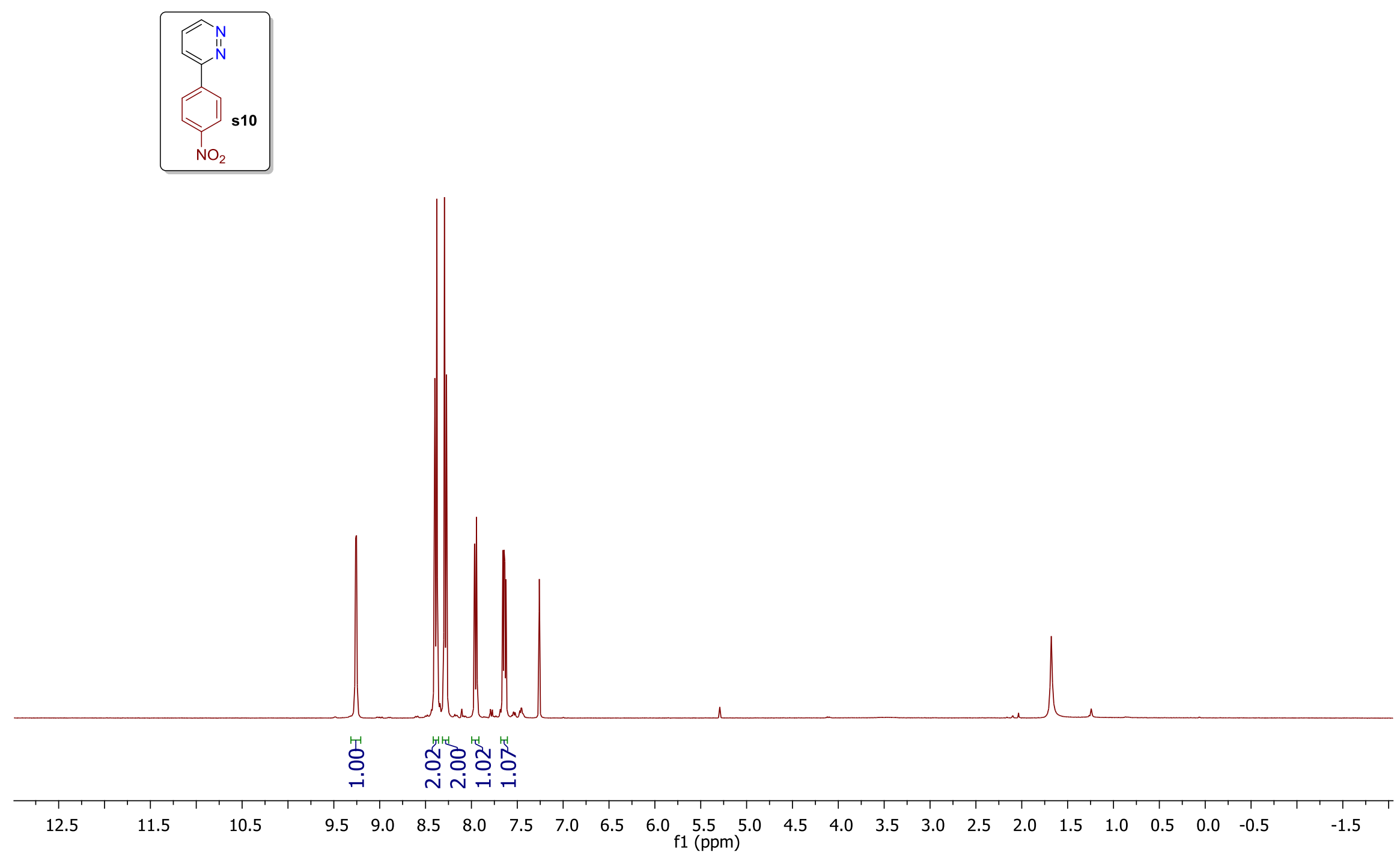


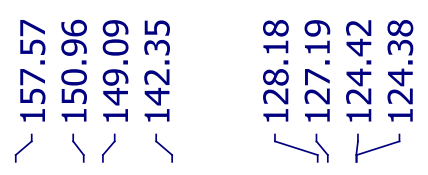
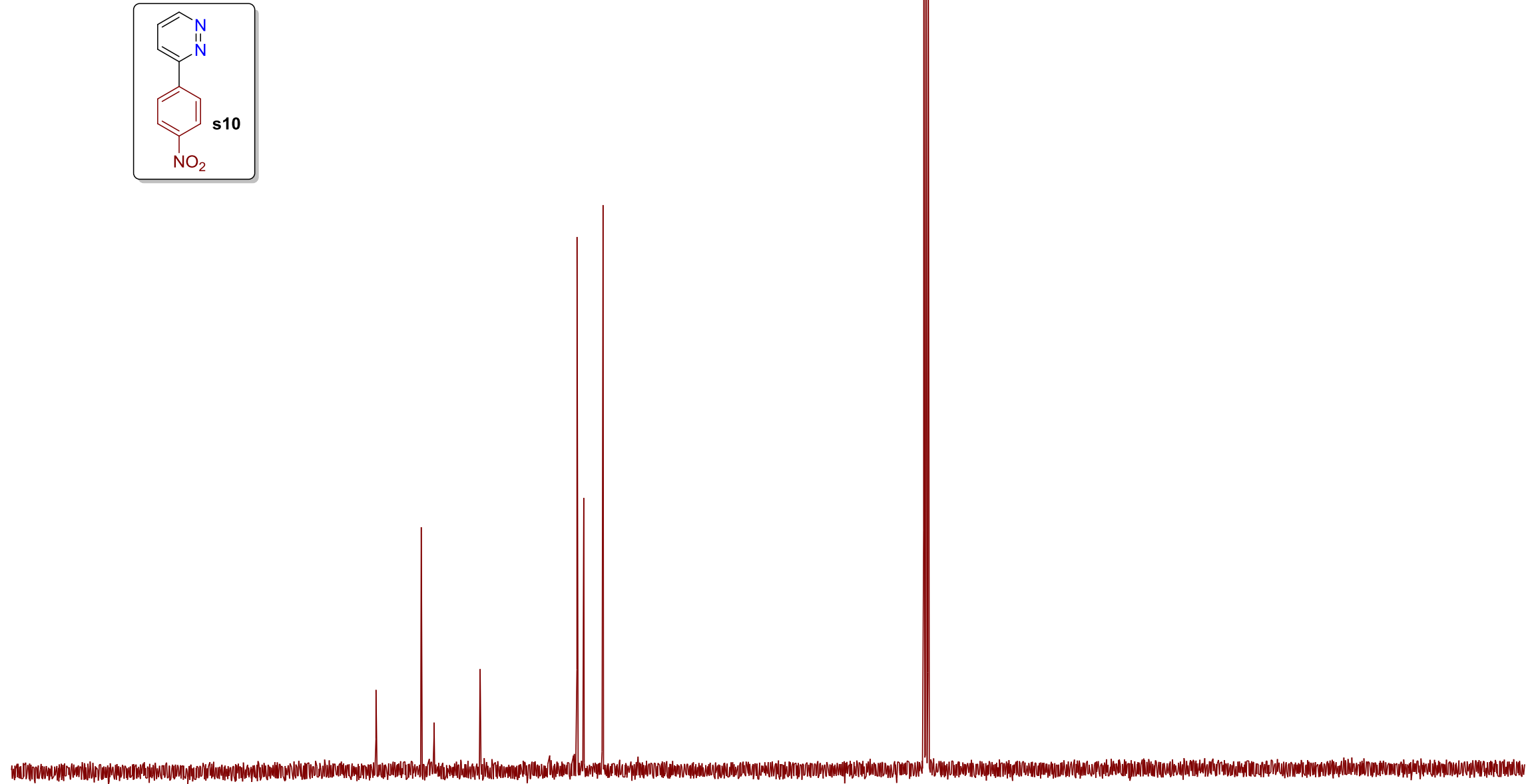

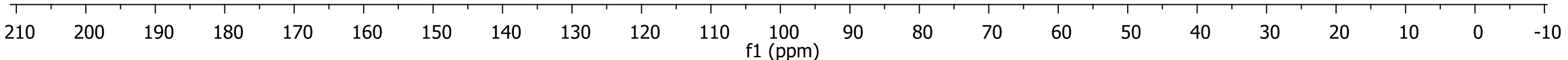




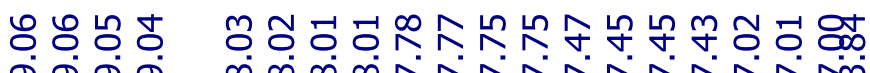

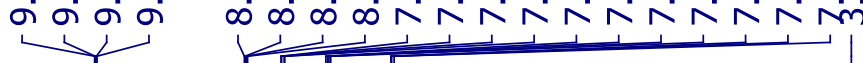

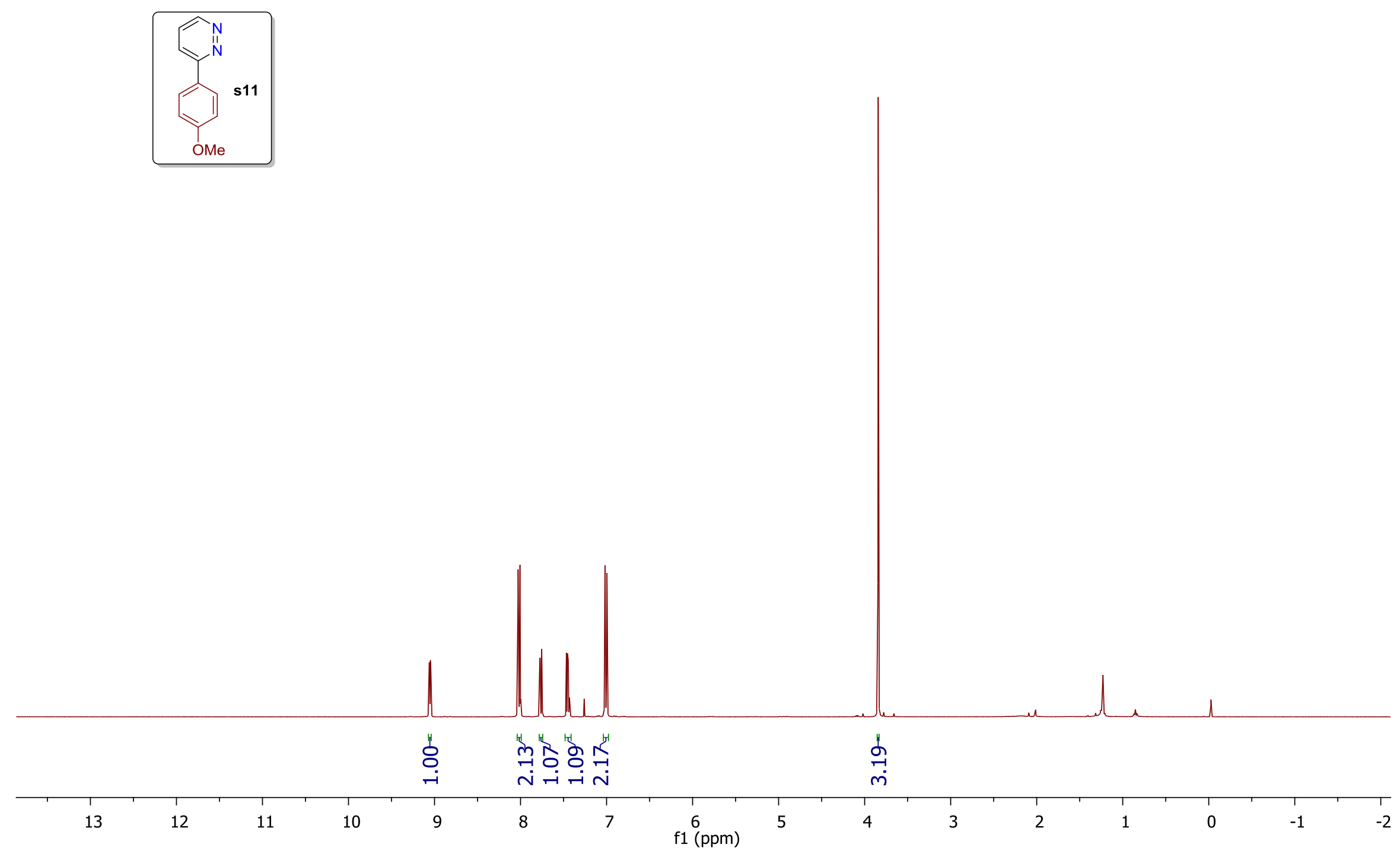




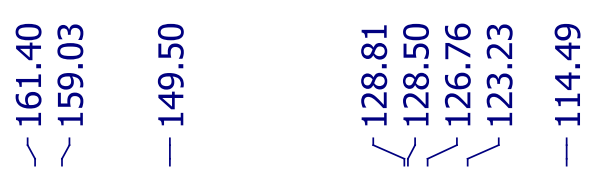

守
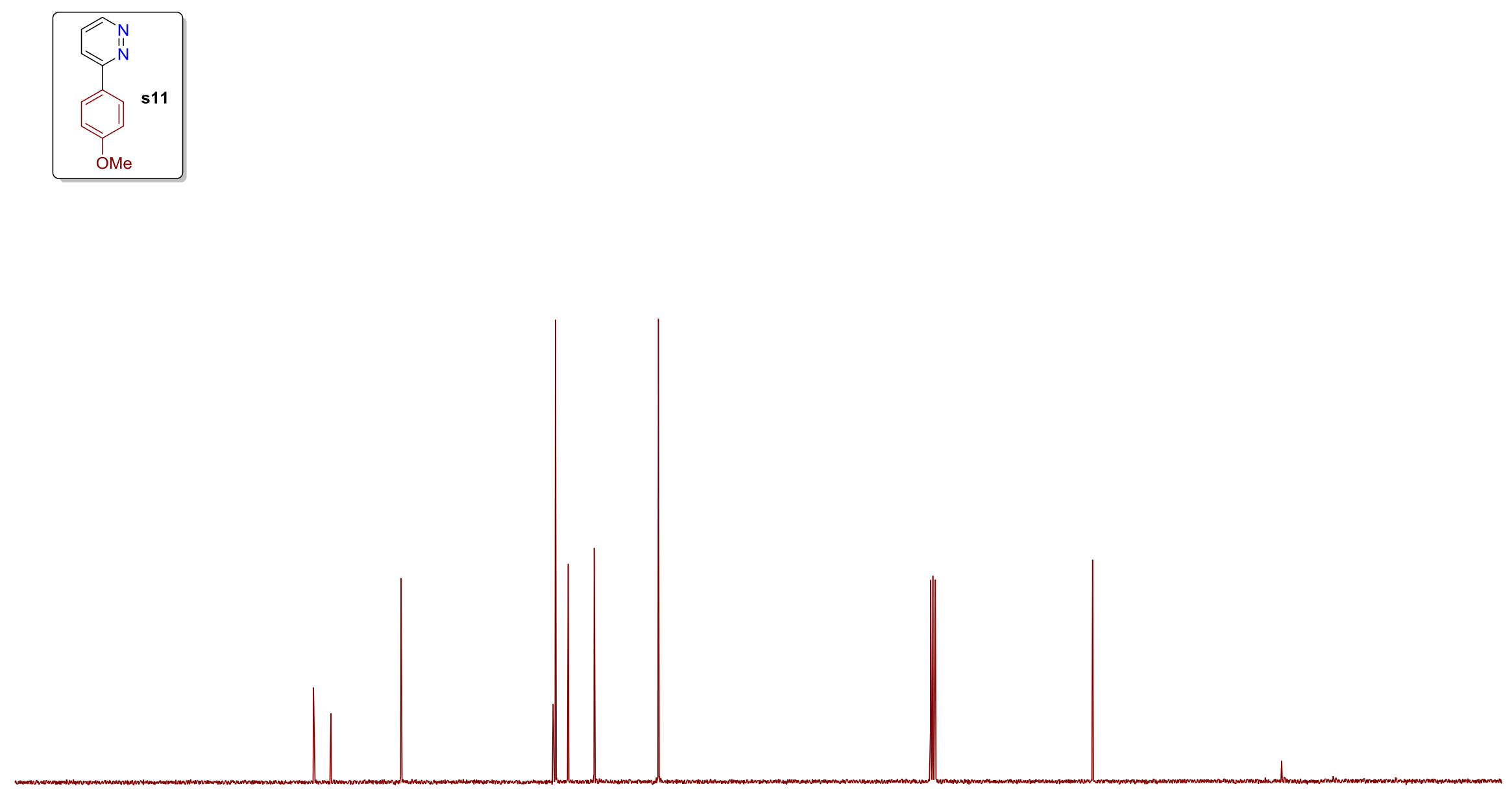


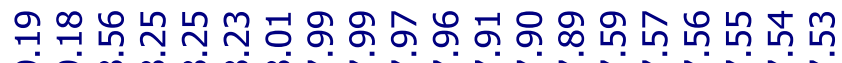

नं
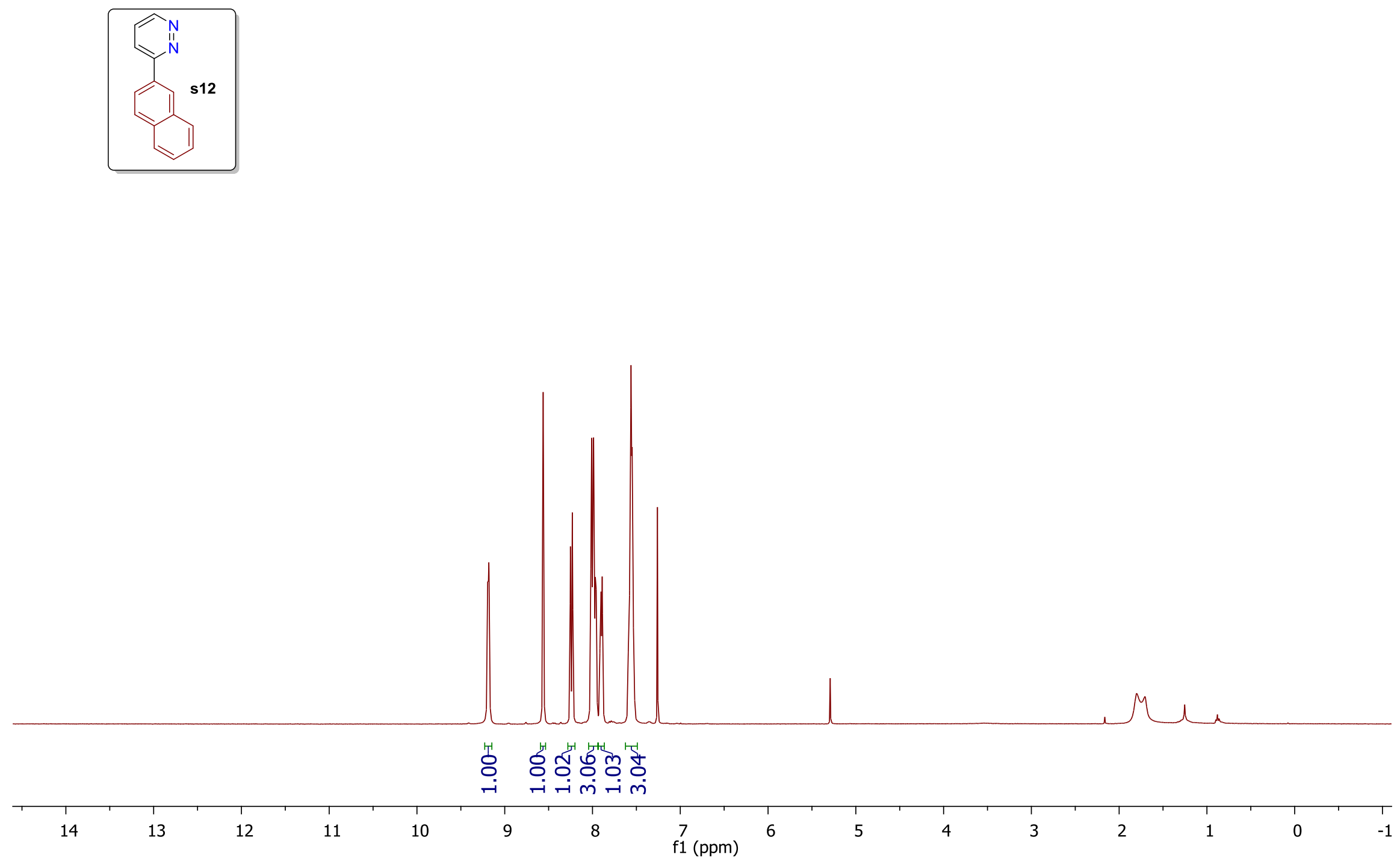

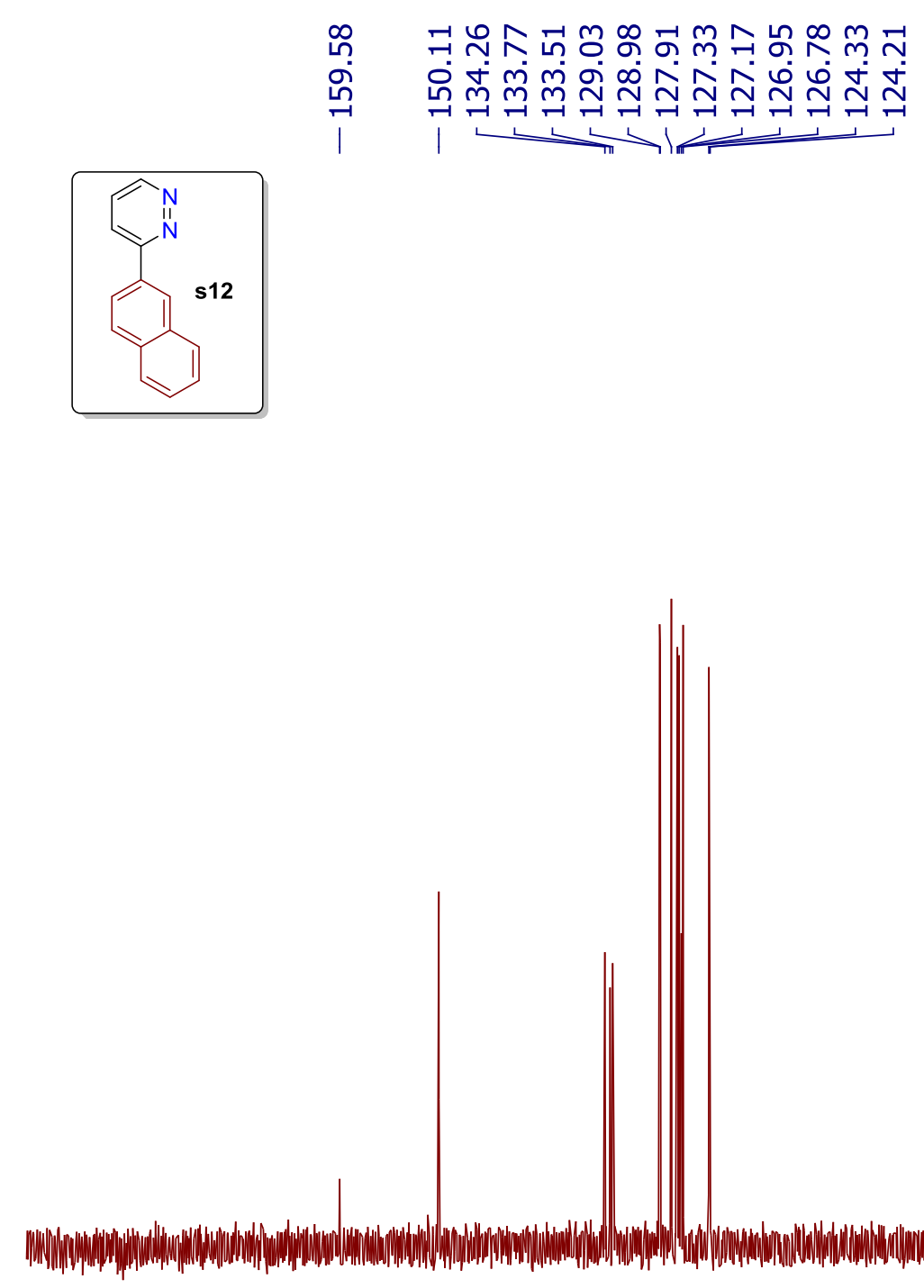

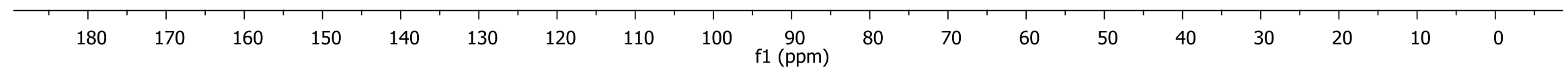




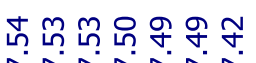

NNNNN

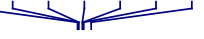

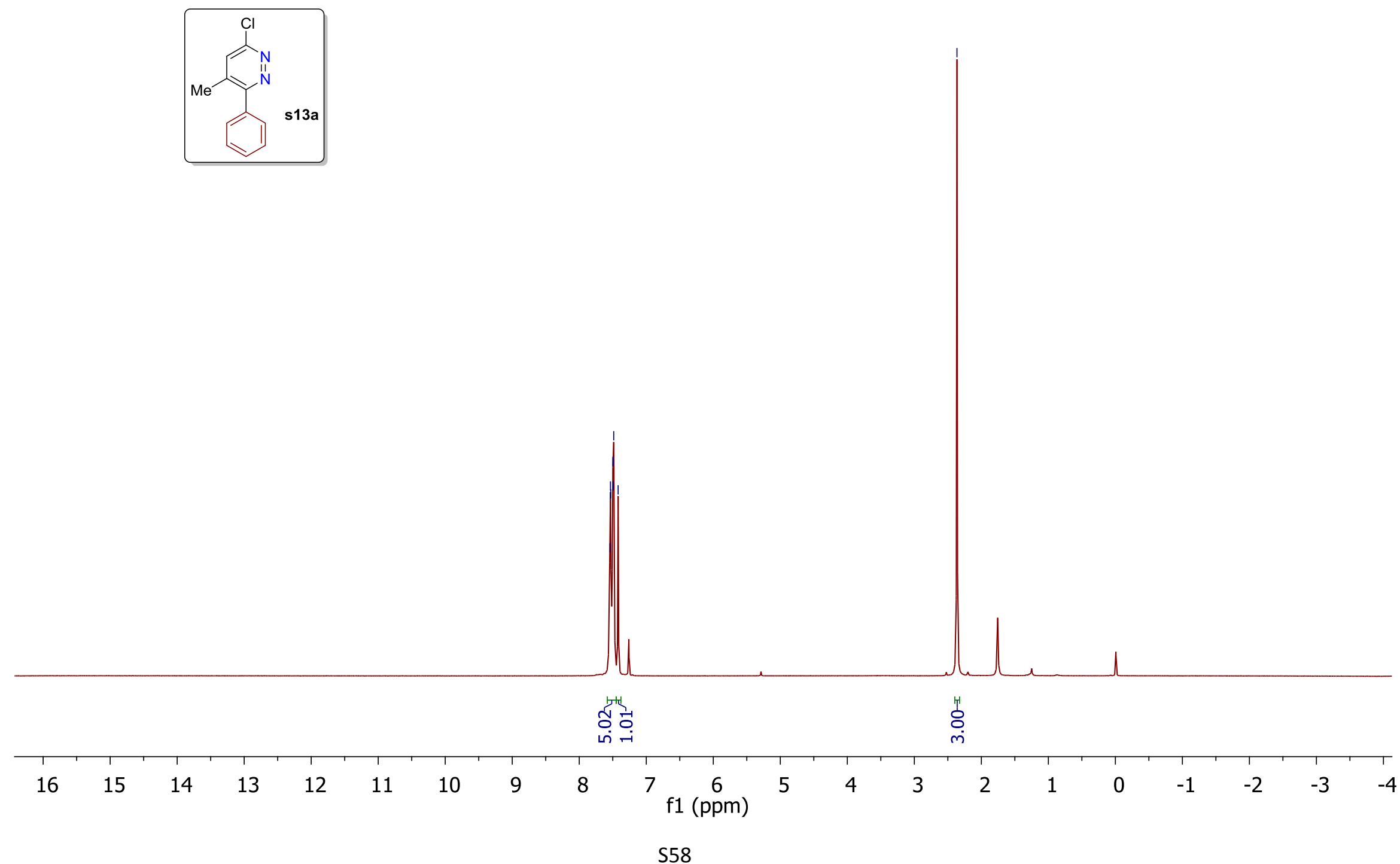

ํำ 


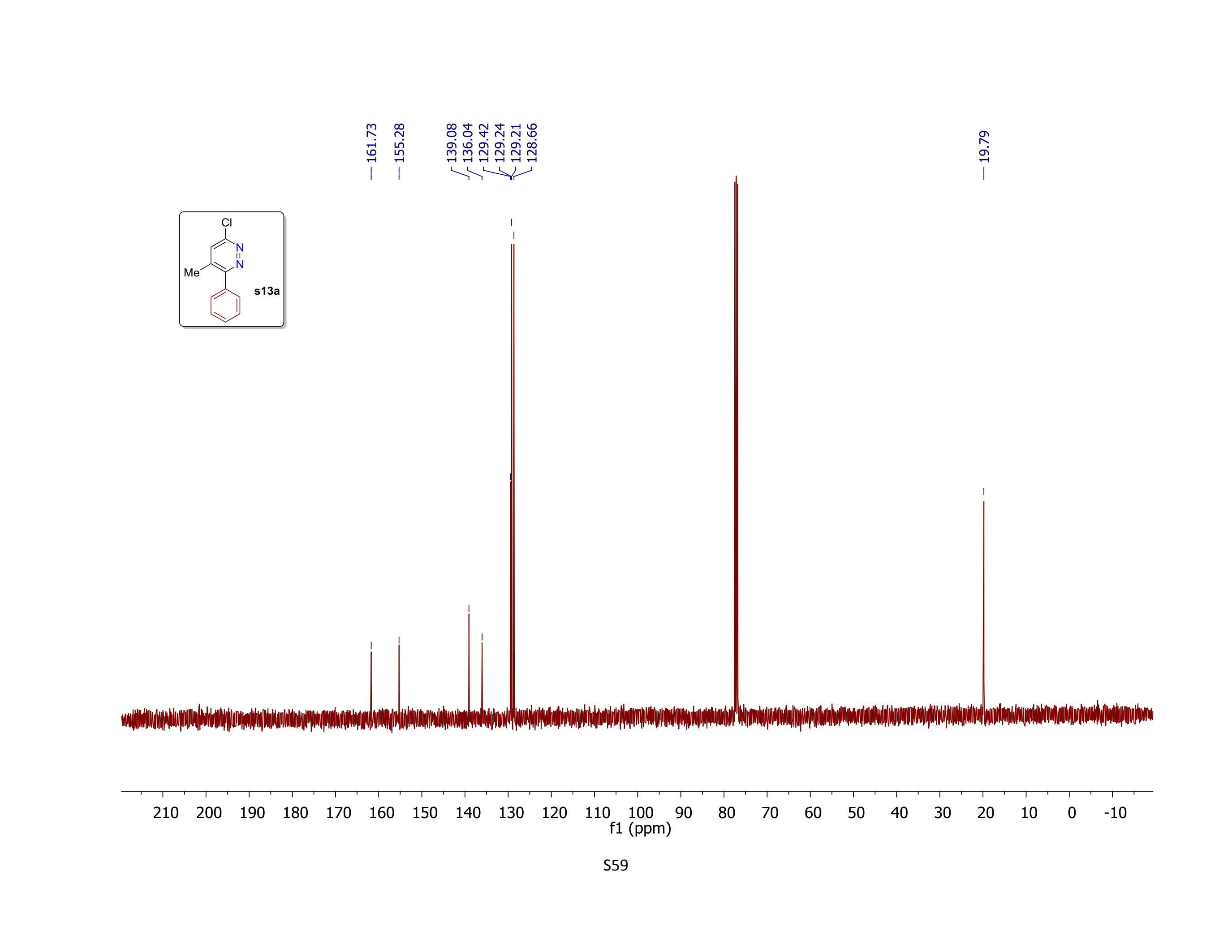




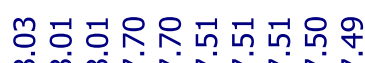

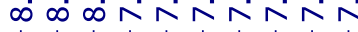

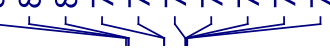

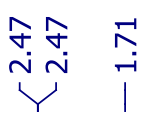

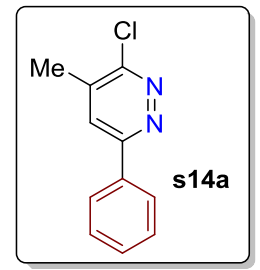

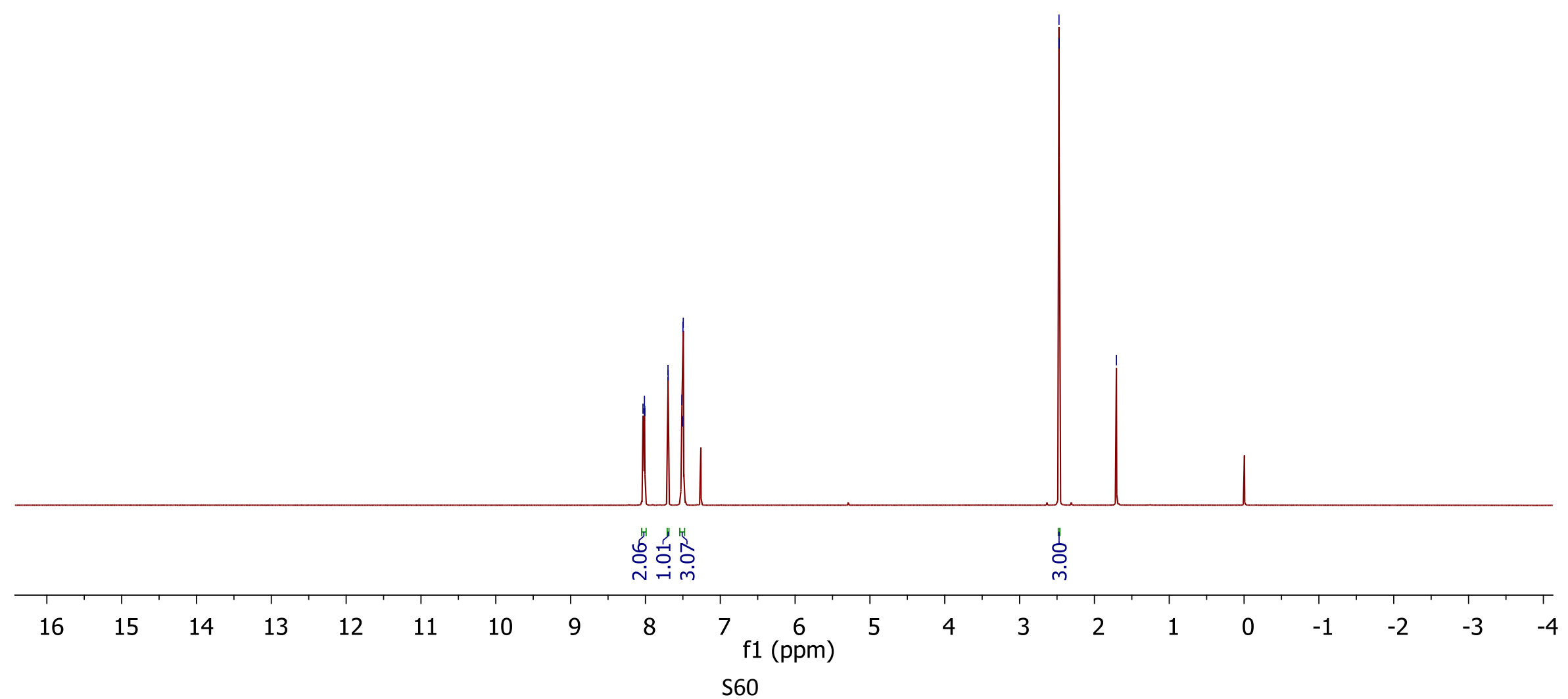




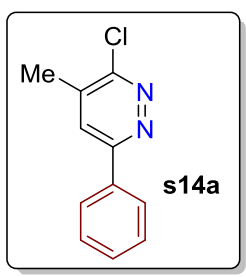

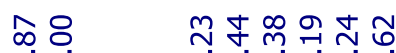

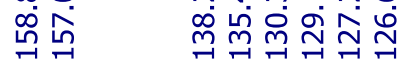

I) परा

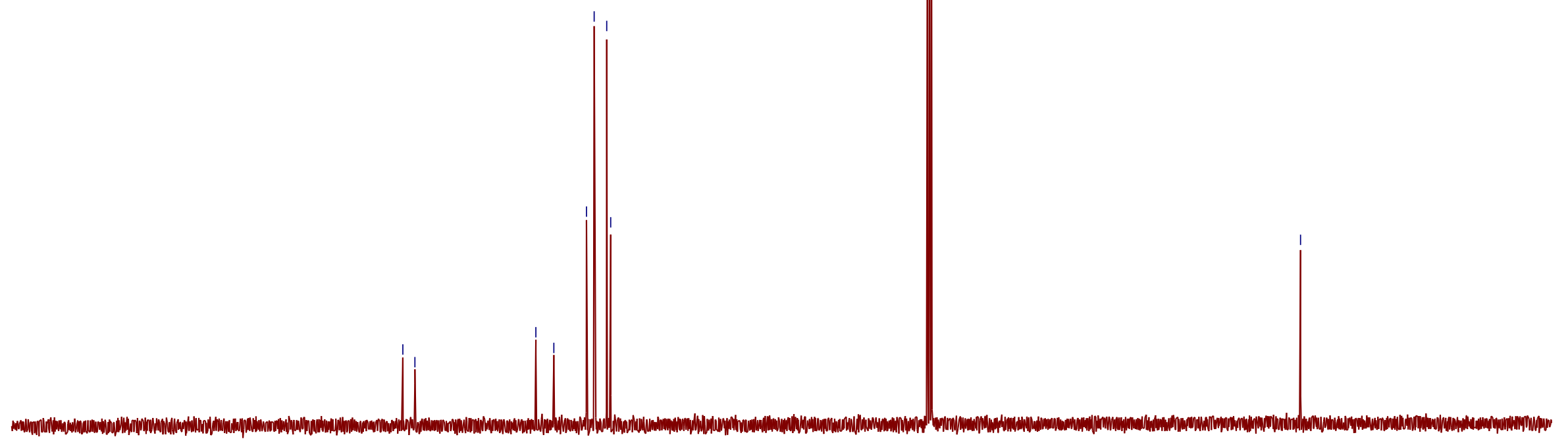

$\begin{array}{llllllllllll}210 & 200 & 190 & 180 & 170 & 160 & 150 & 140 & 130 & 120 & 110 & 100 \\ \mathrm{f} 1(\mathrm{ppm})\end{array}$

$80 \quad 70$ S61 


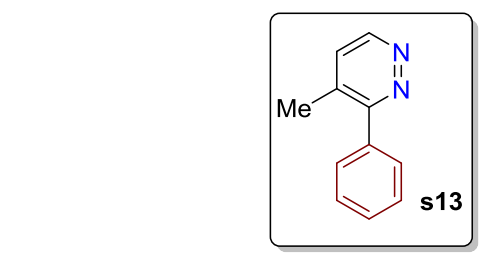

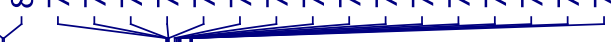

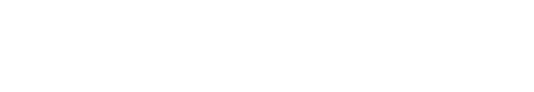

. 

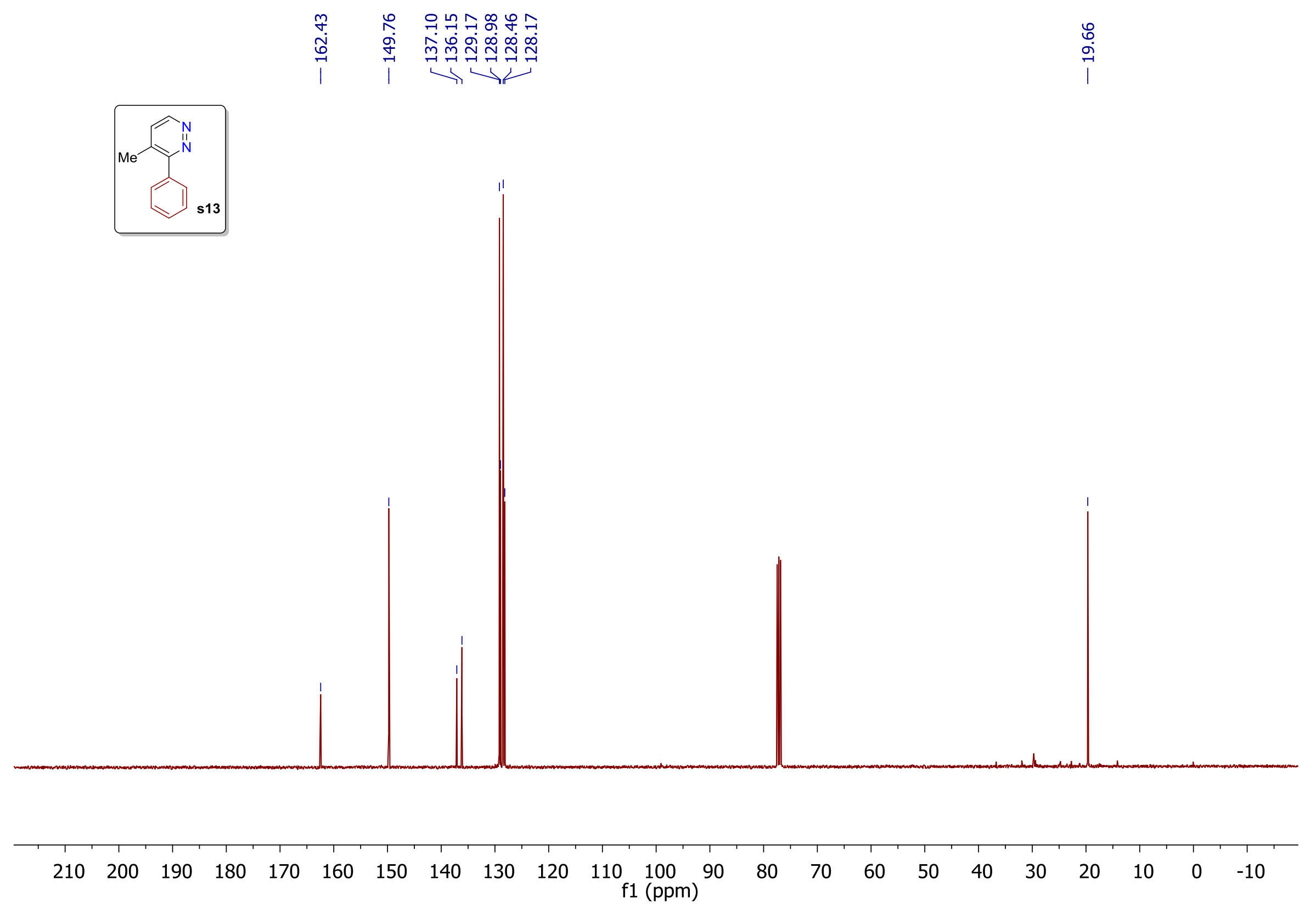


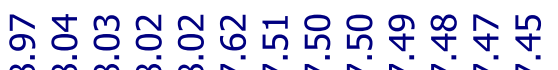

$\infty \infty^{\circ} \infty \infty^{\circ} \mathbf{N} N \mathbf{N} N$
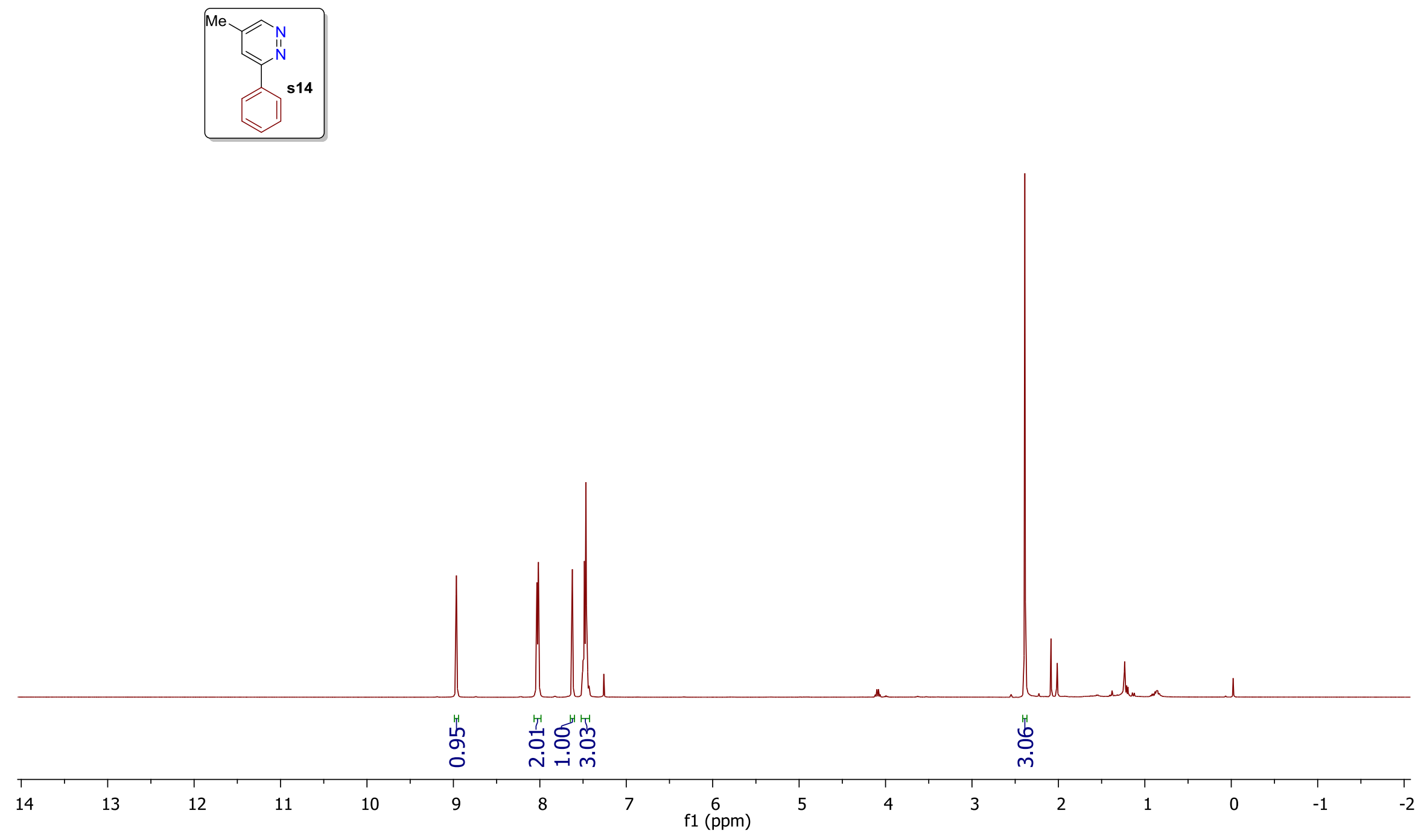


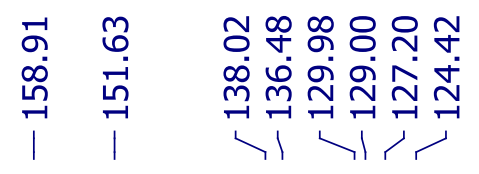

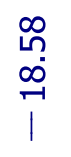
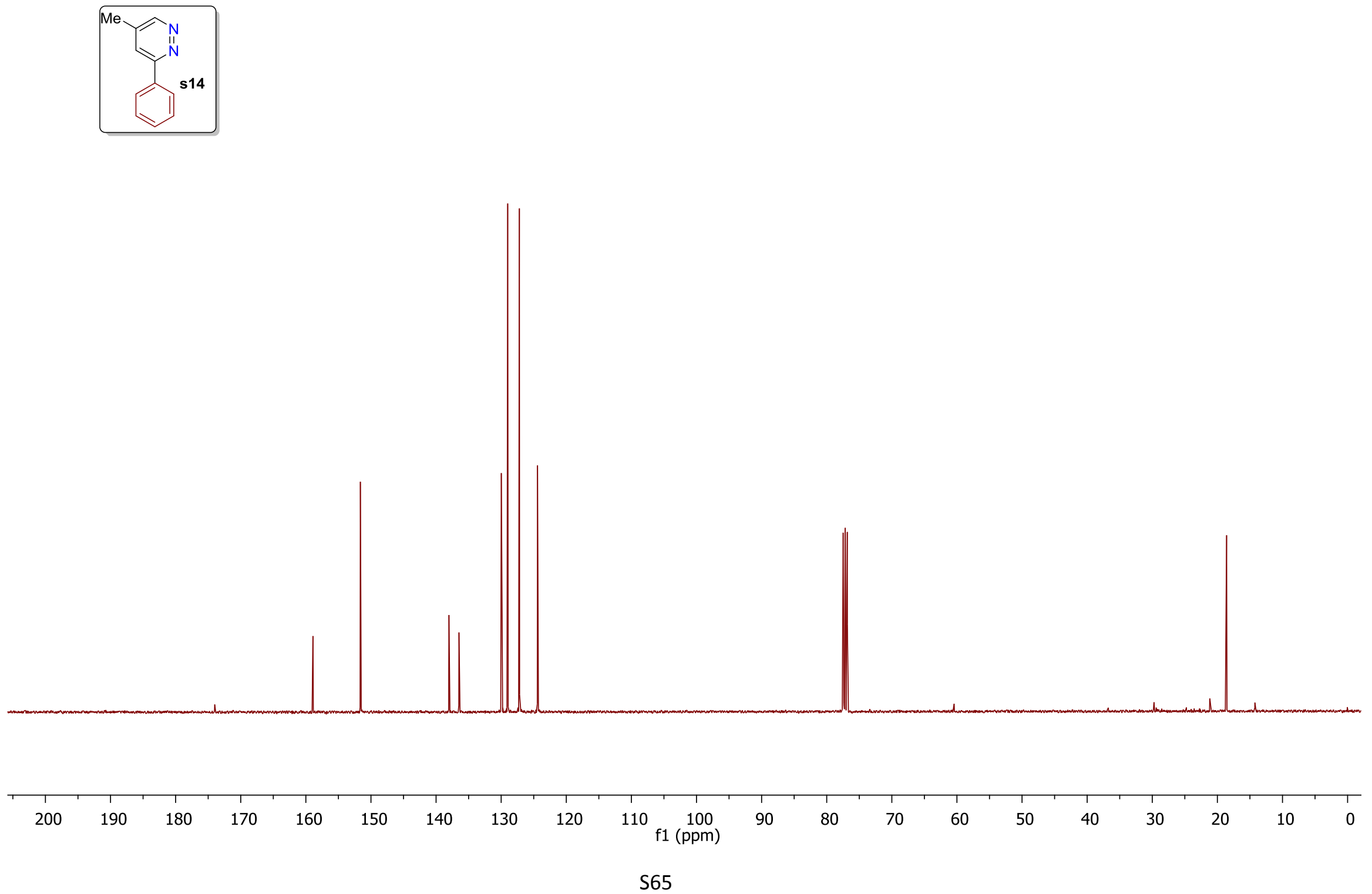


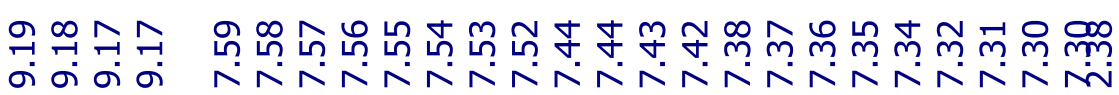

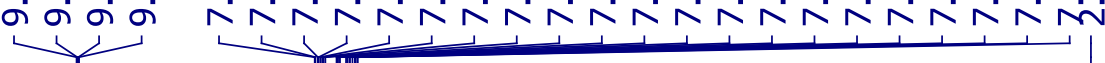
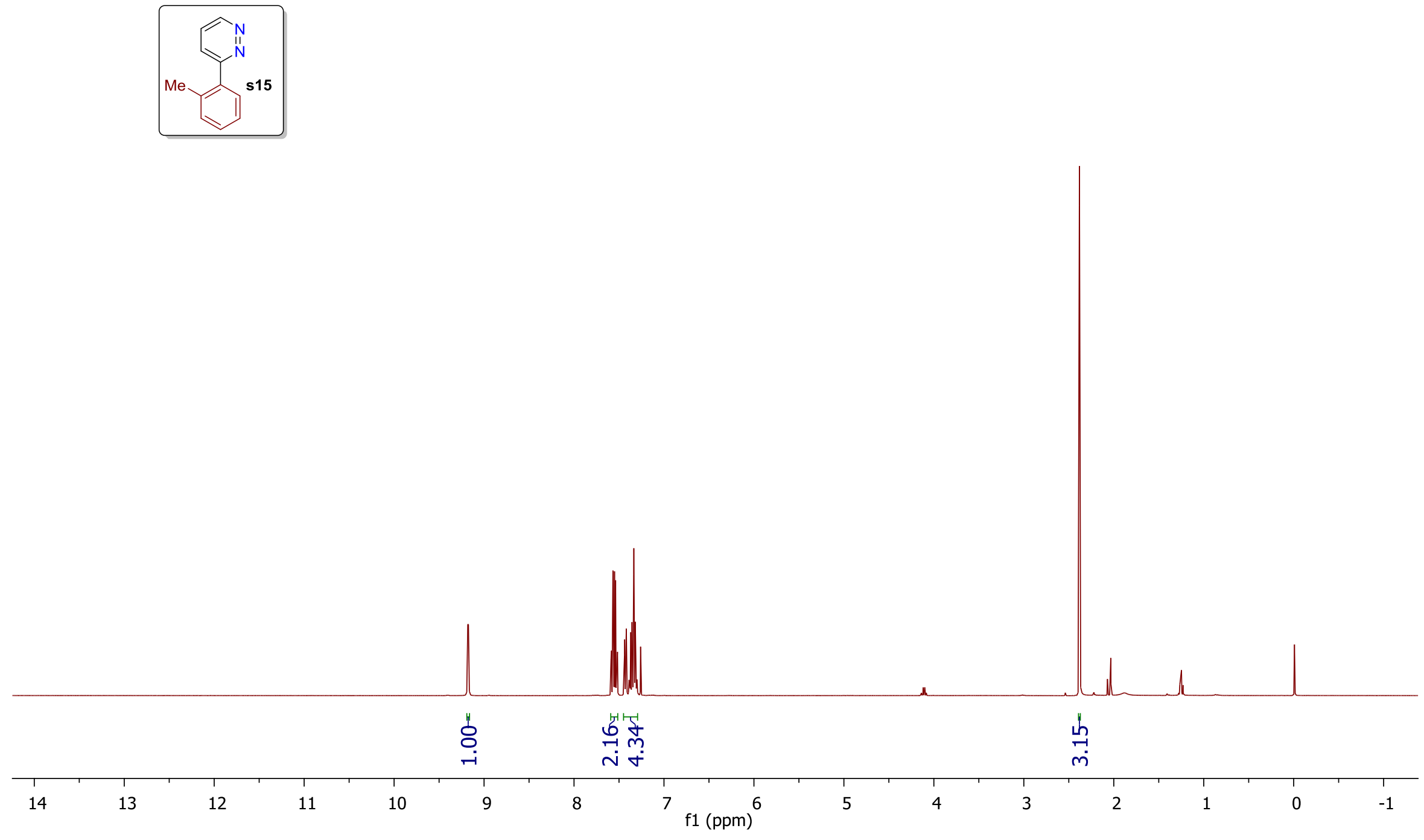

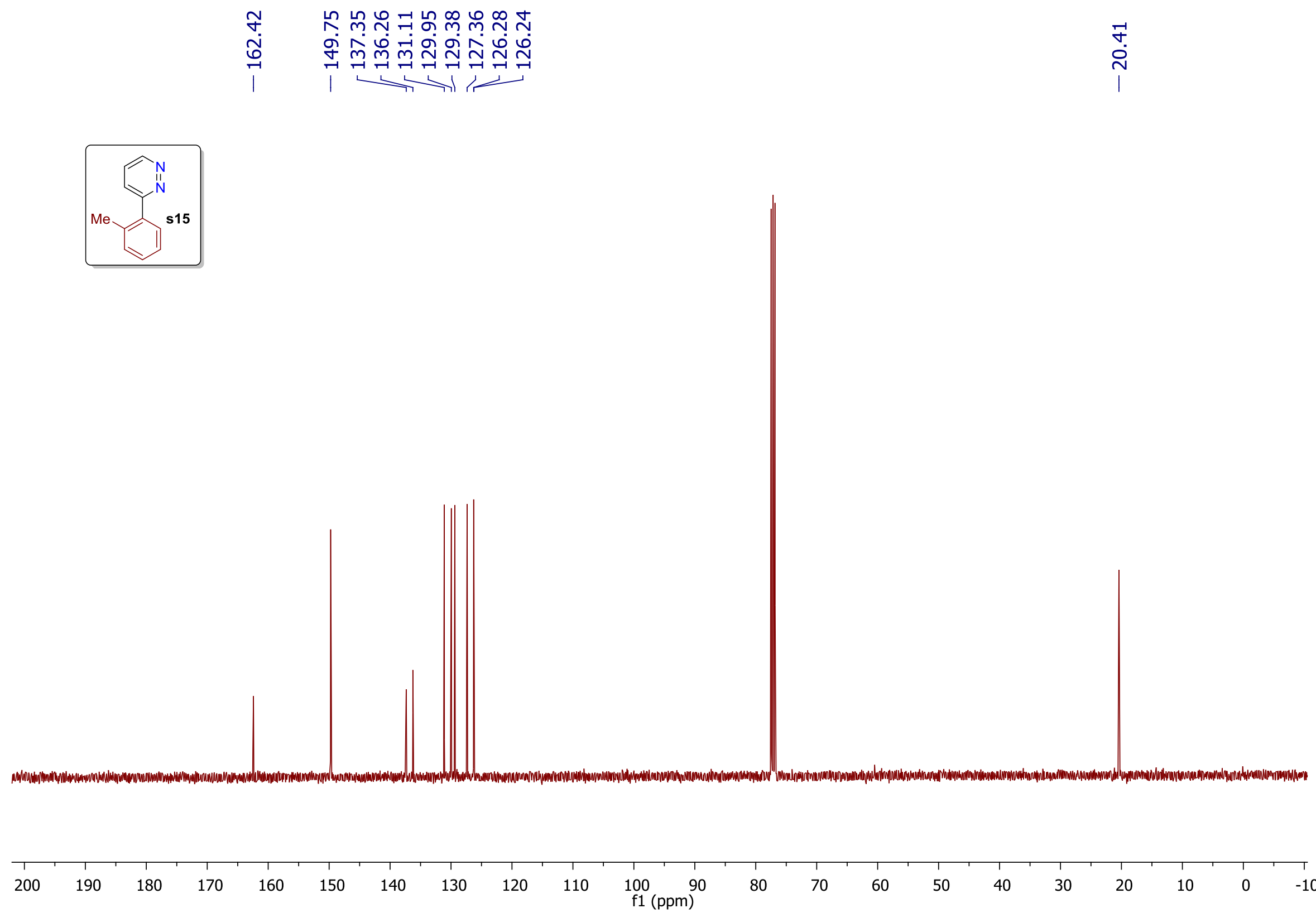


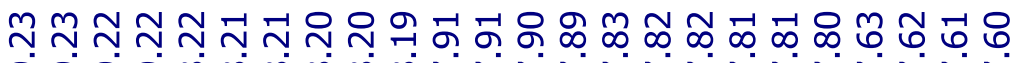

नं $\sigma^{\infty} \infty \infty_{\infty}^{\infty} \infty \infty^{\infty} N$ NNNNNNNNNN
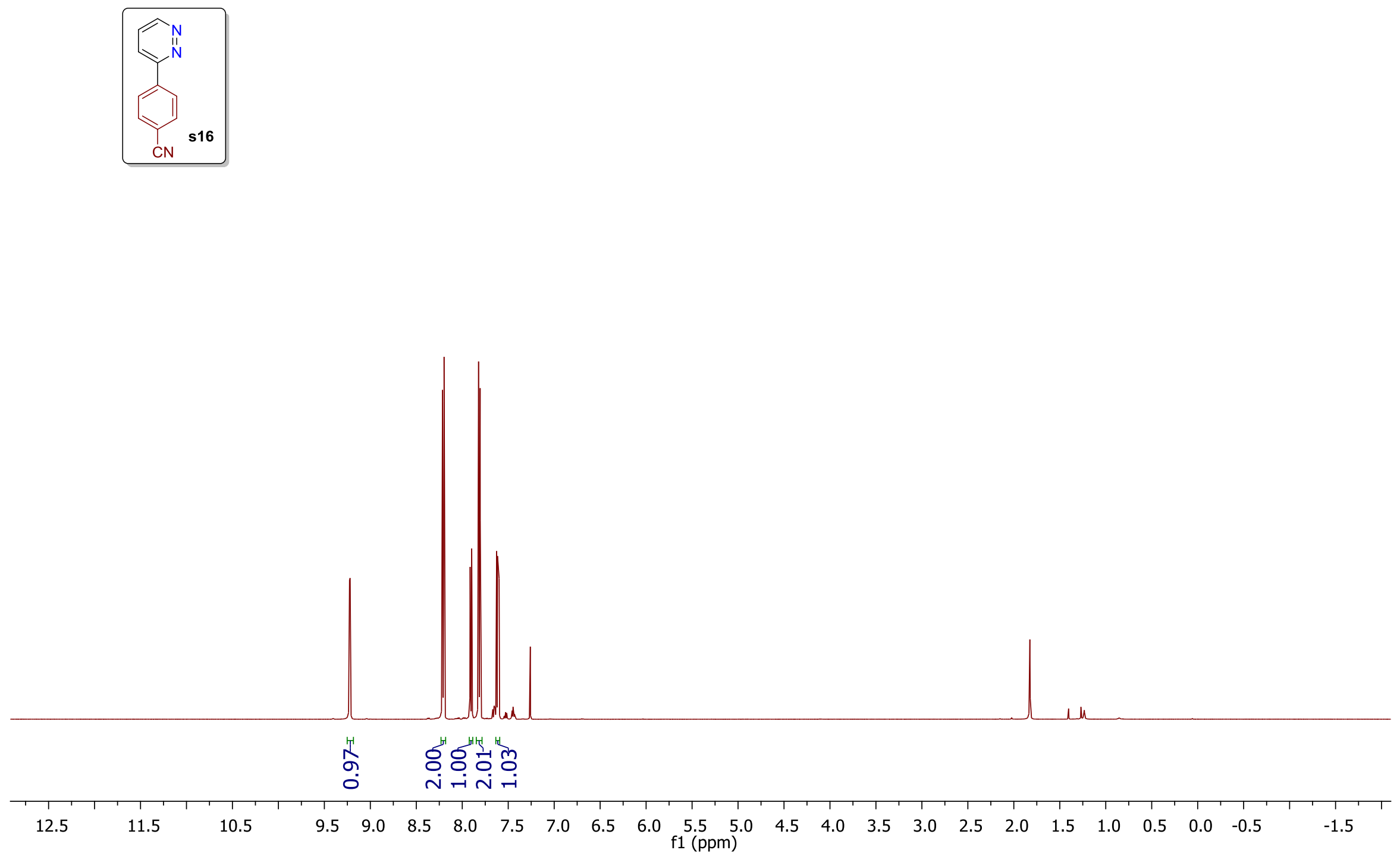


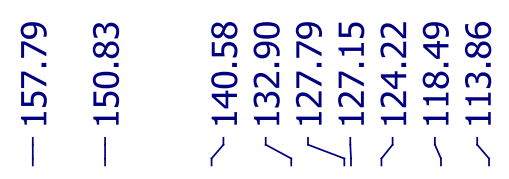
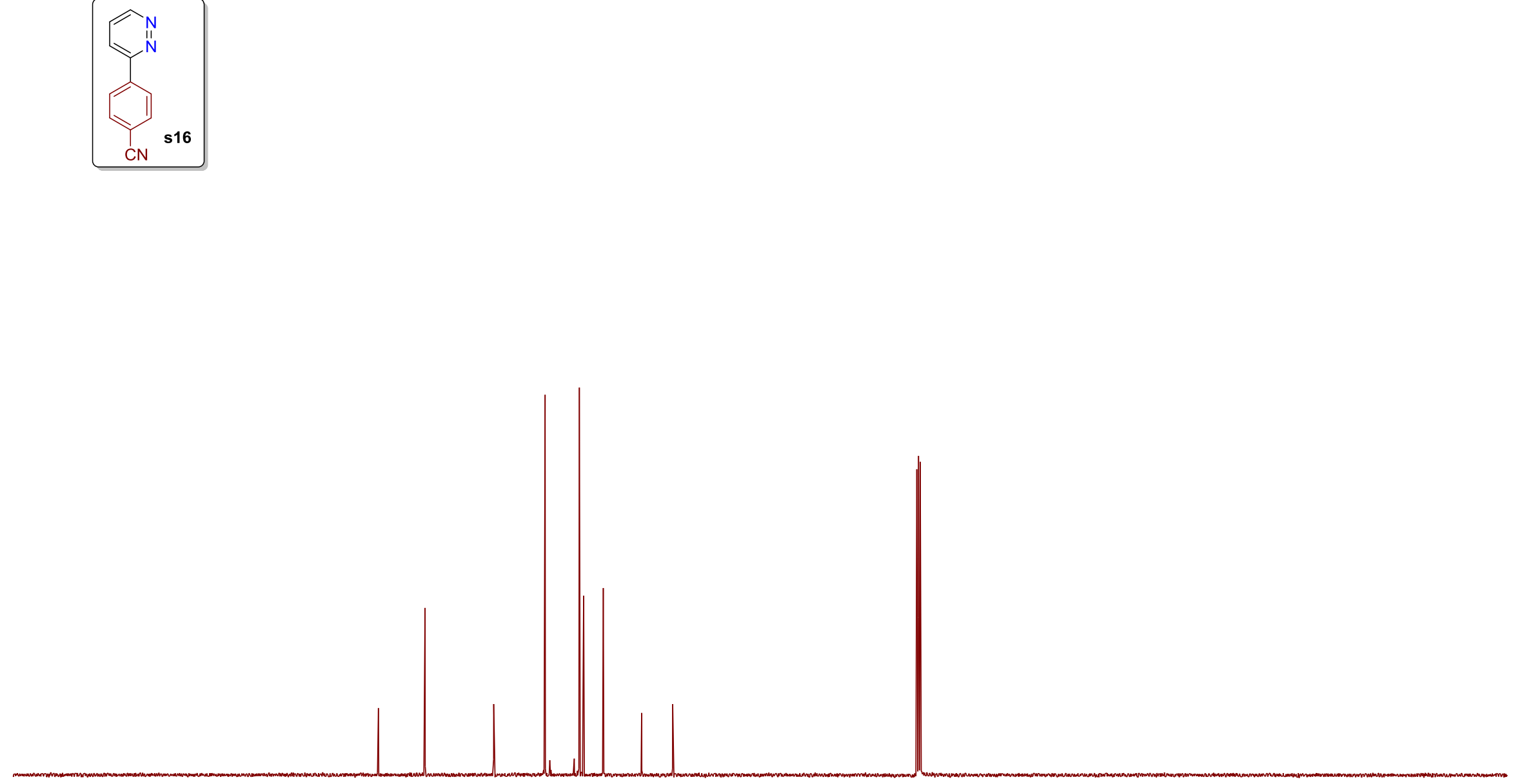

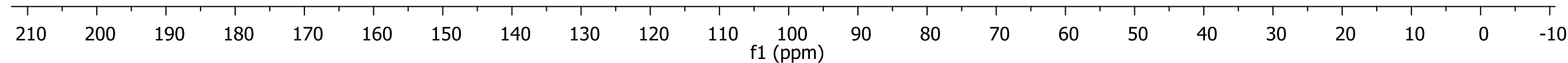


ำ

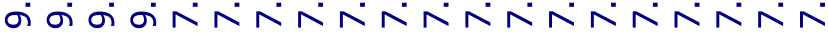

î
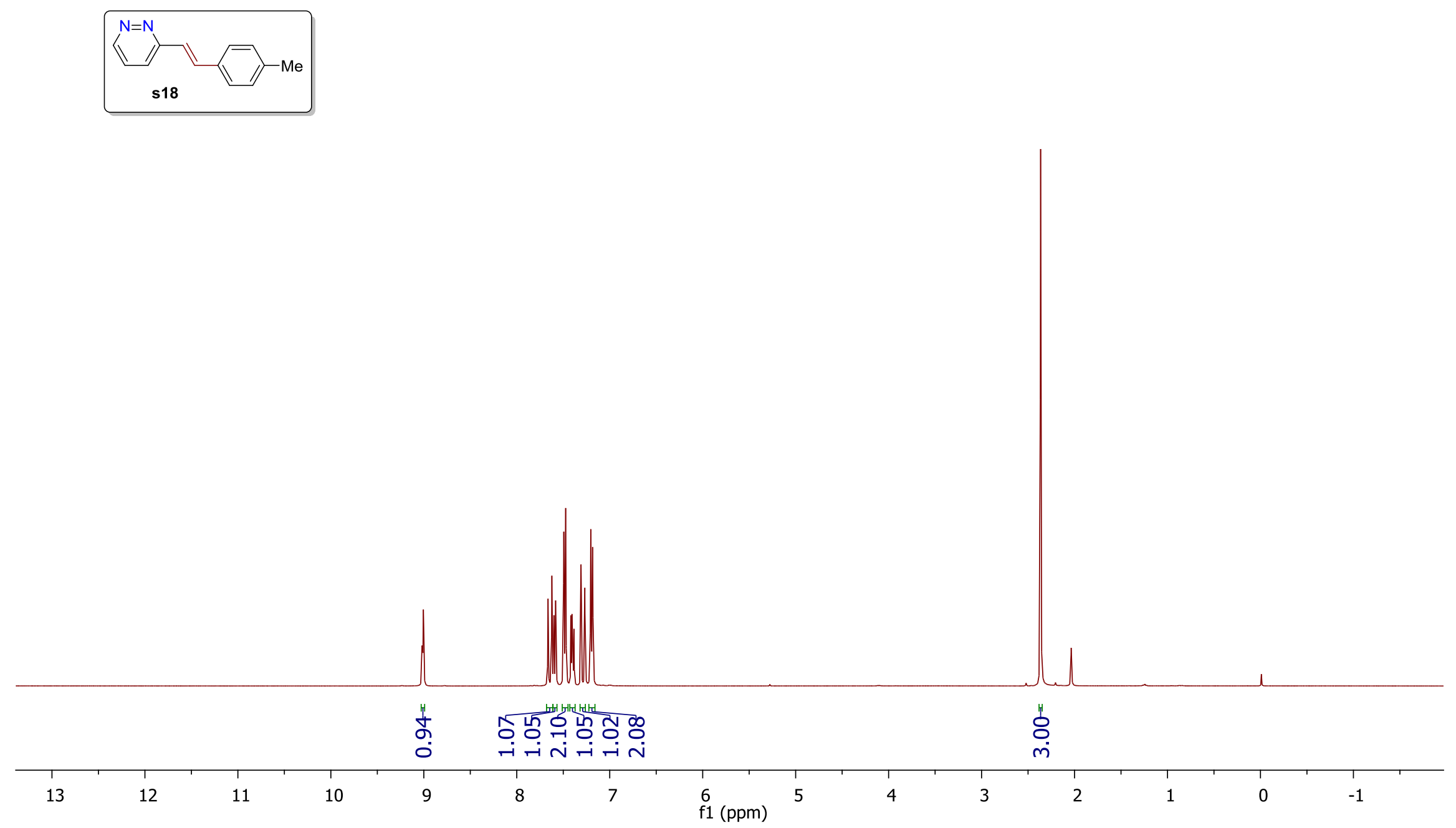

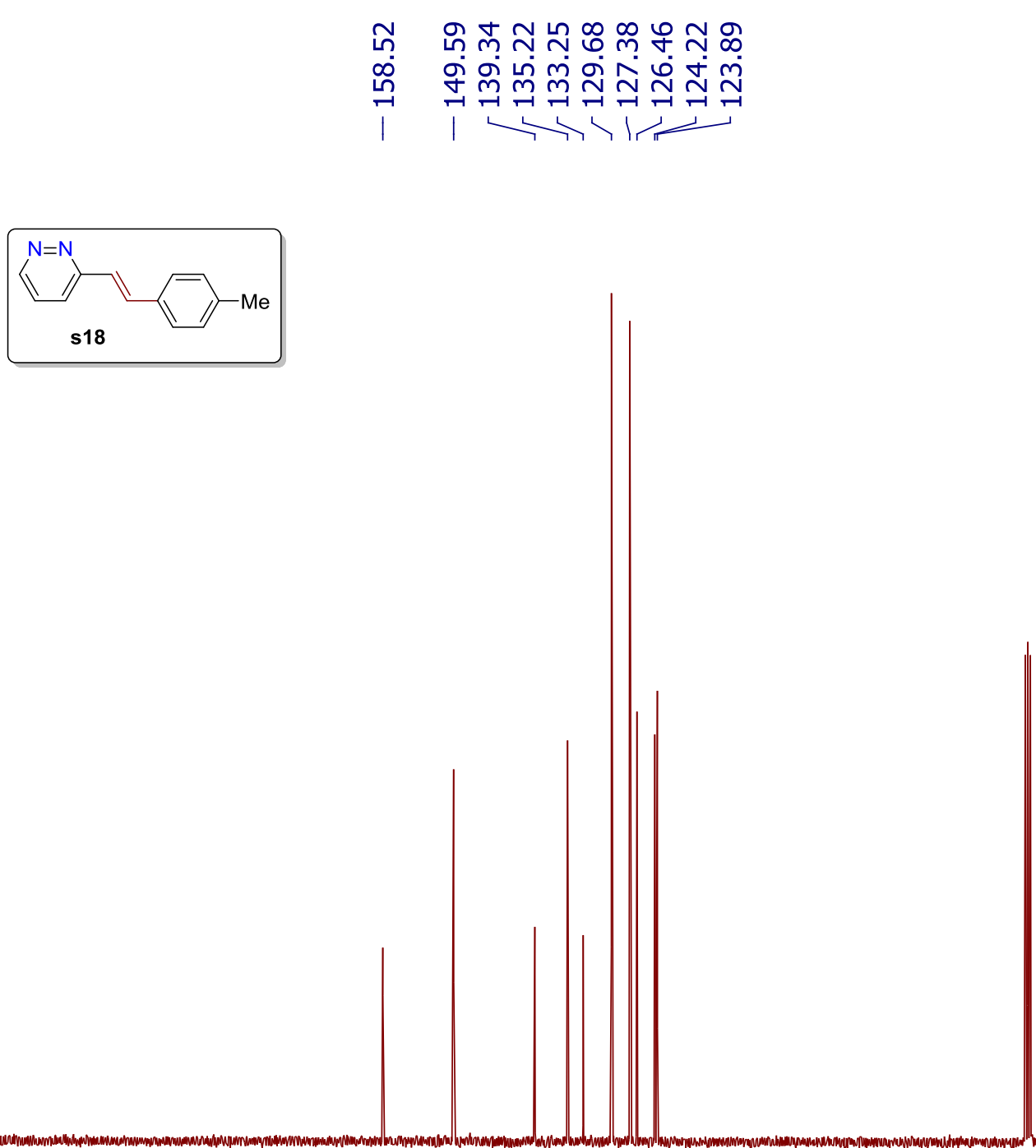

$210 \quad 200$ 


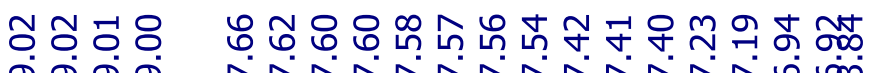

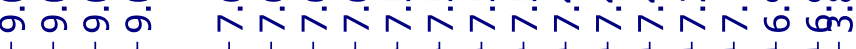

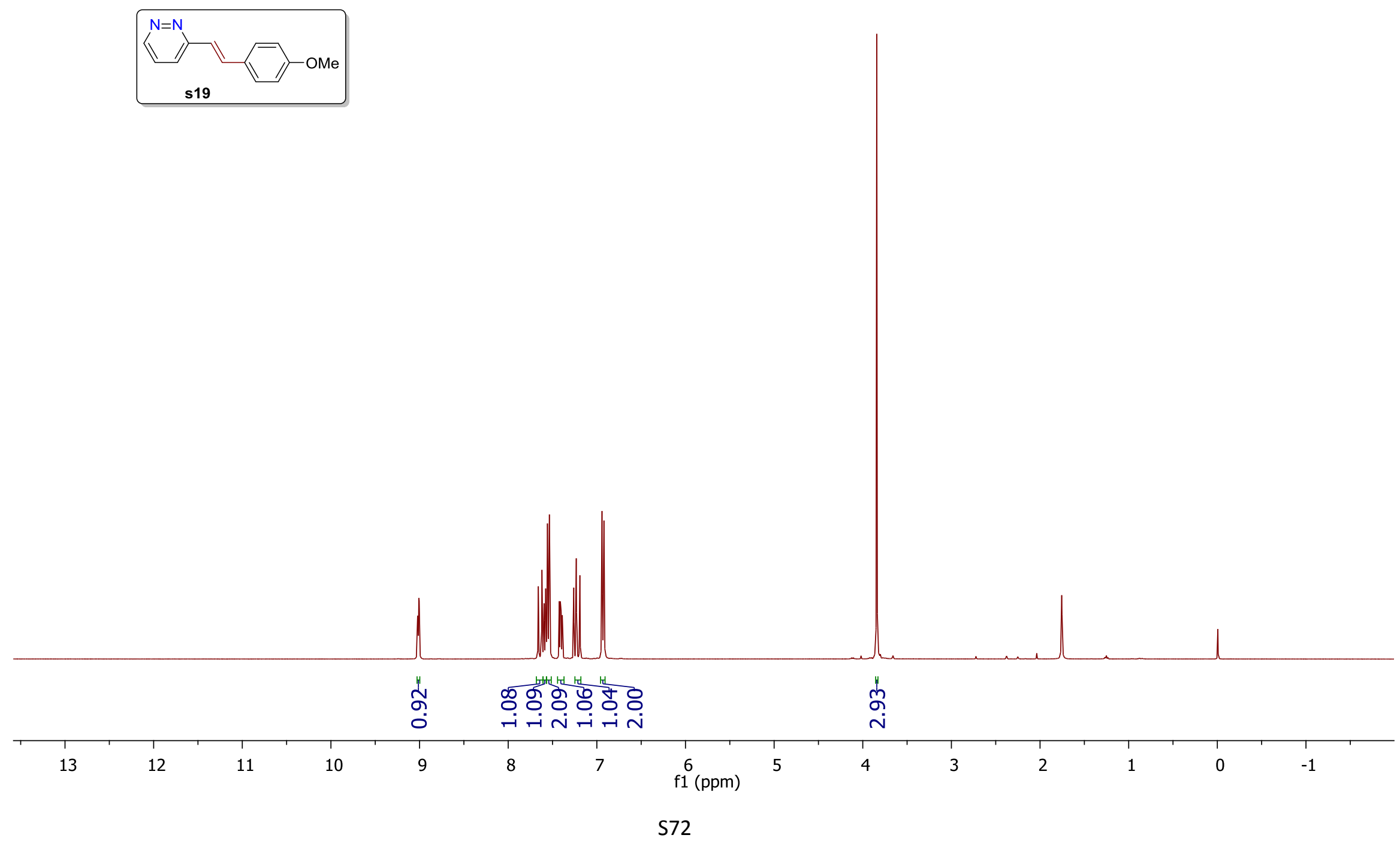

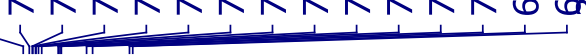




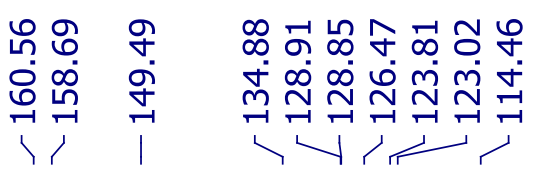

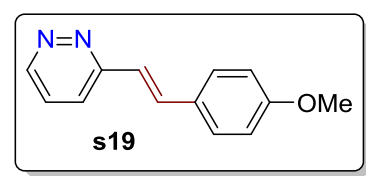

우

กก

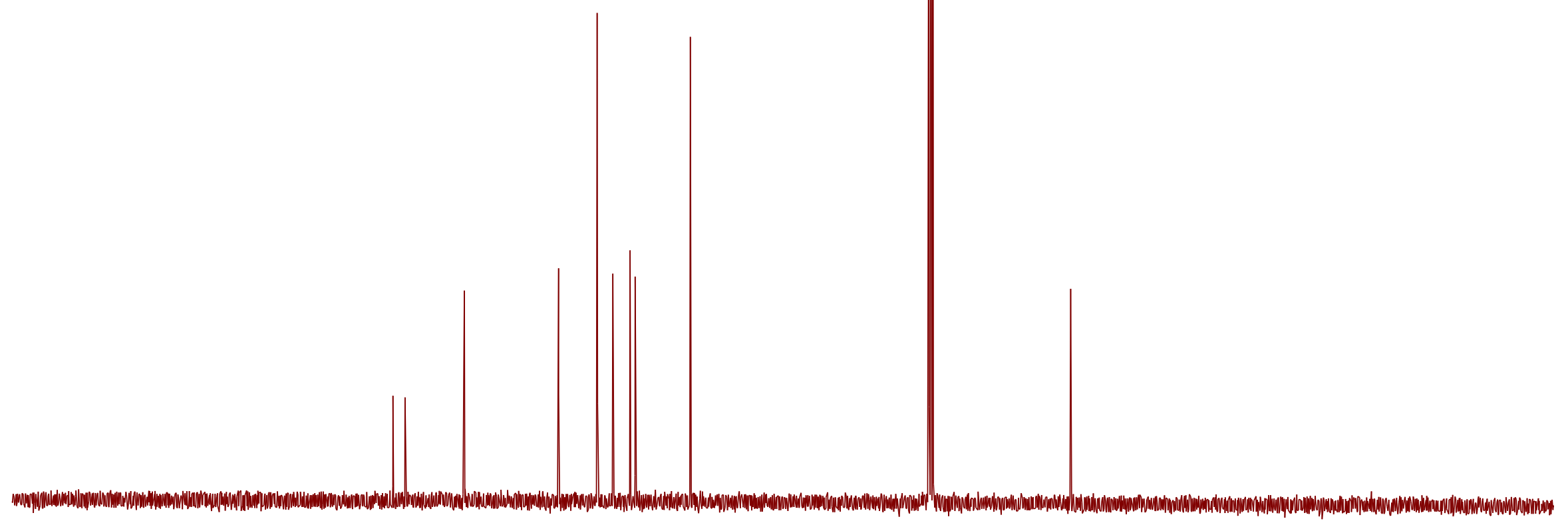

$210 \quad 200$ 


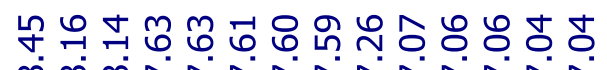

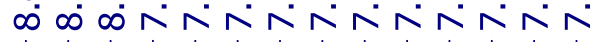

$\overbrace{N}^{N^{-O^{-}}}{ }_{9 a}$

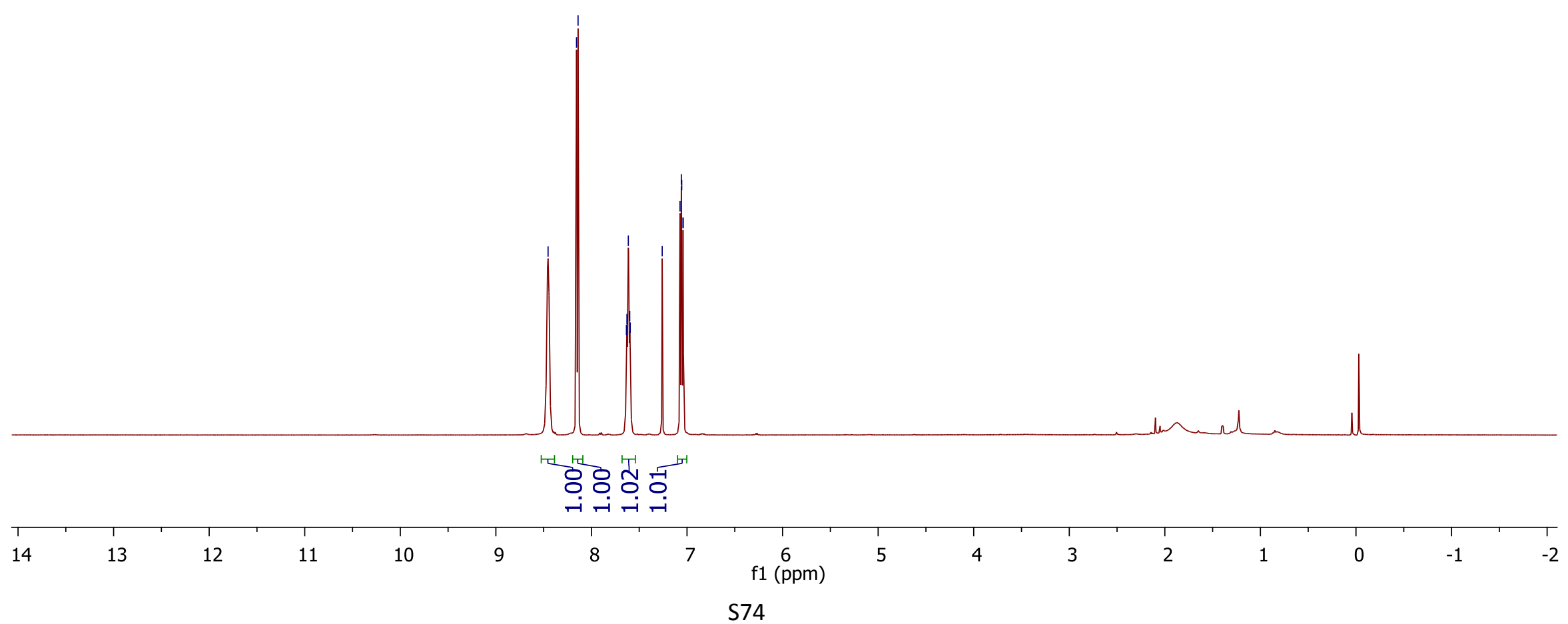


I

$$
\overbrace{9 a}^{+N^{-}}
$$

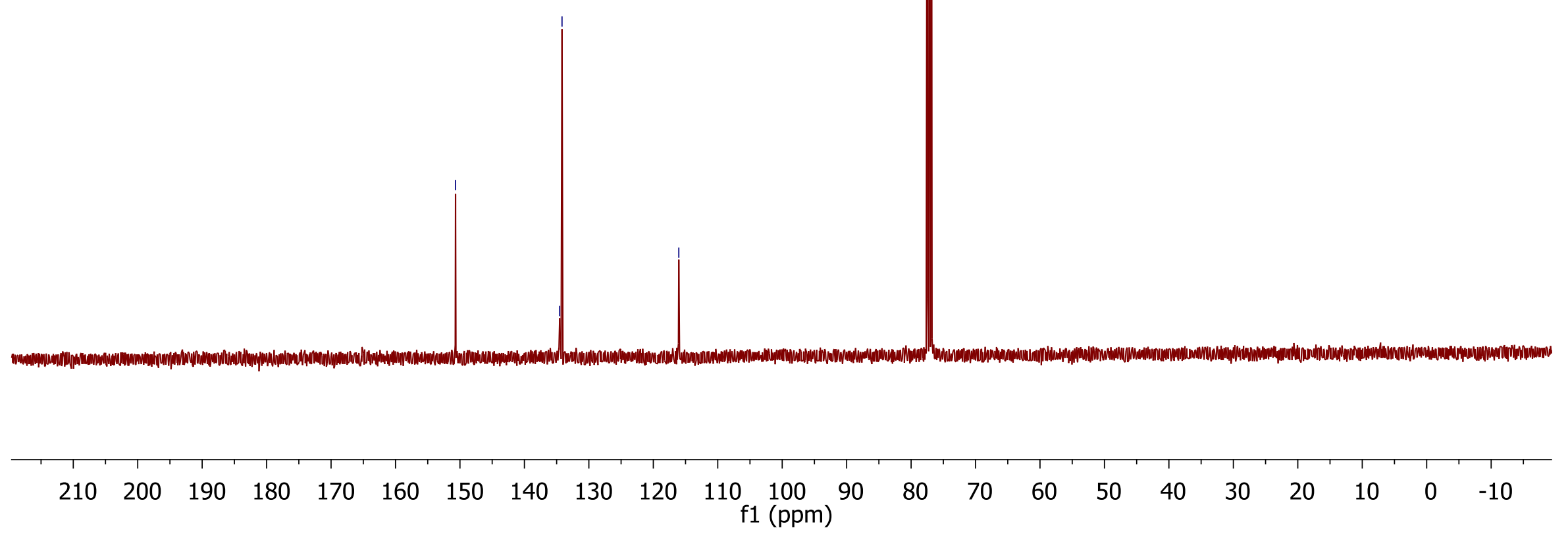




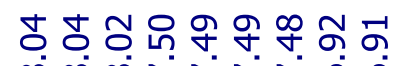

$\infty \infty \infty \wedge N \wedge 0^{\circ}$

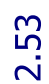

$$
\underbrace{N_{N}^{+}}_{\text {Me } 9 b}
$$

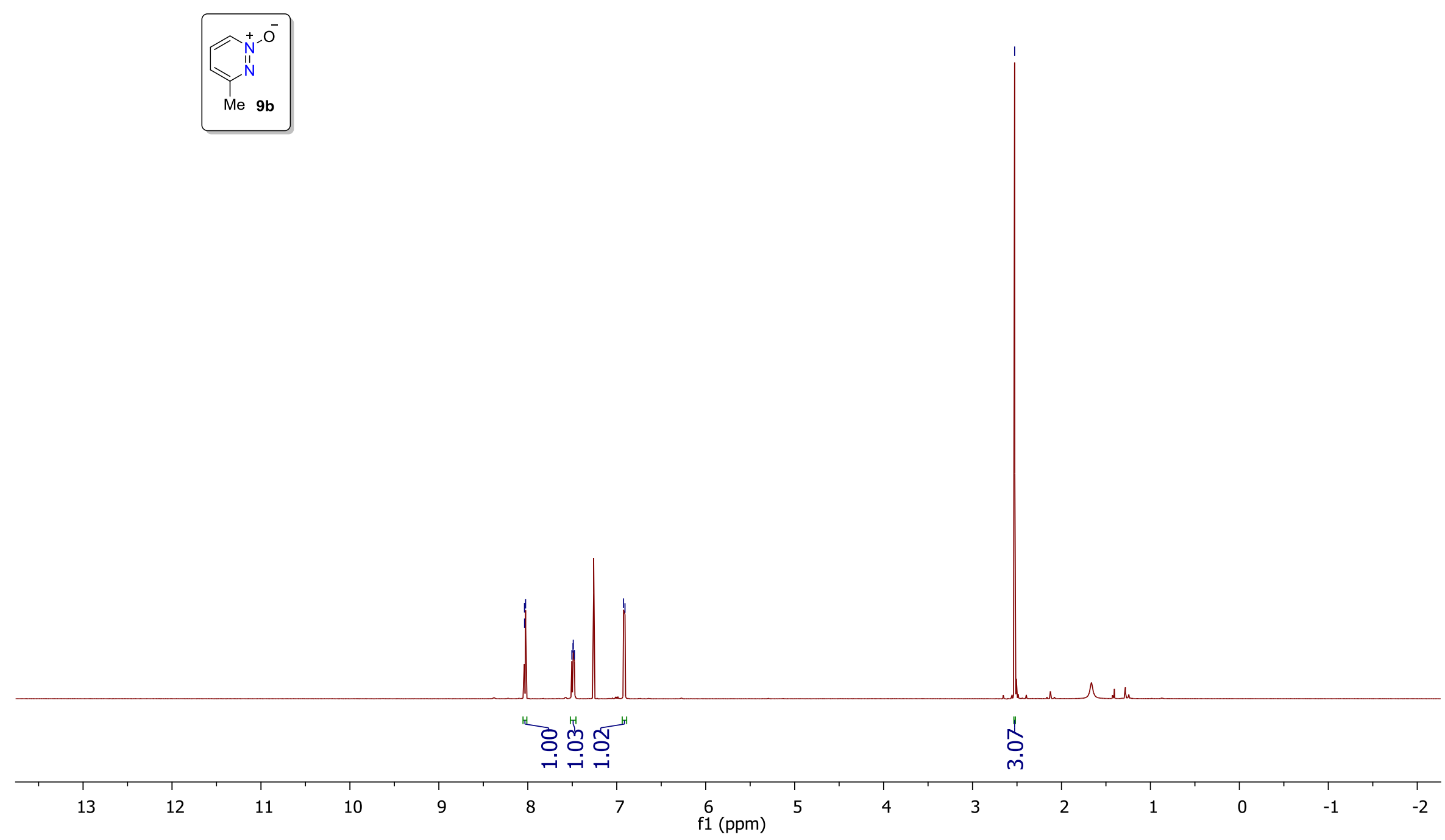



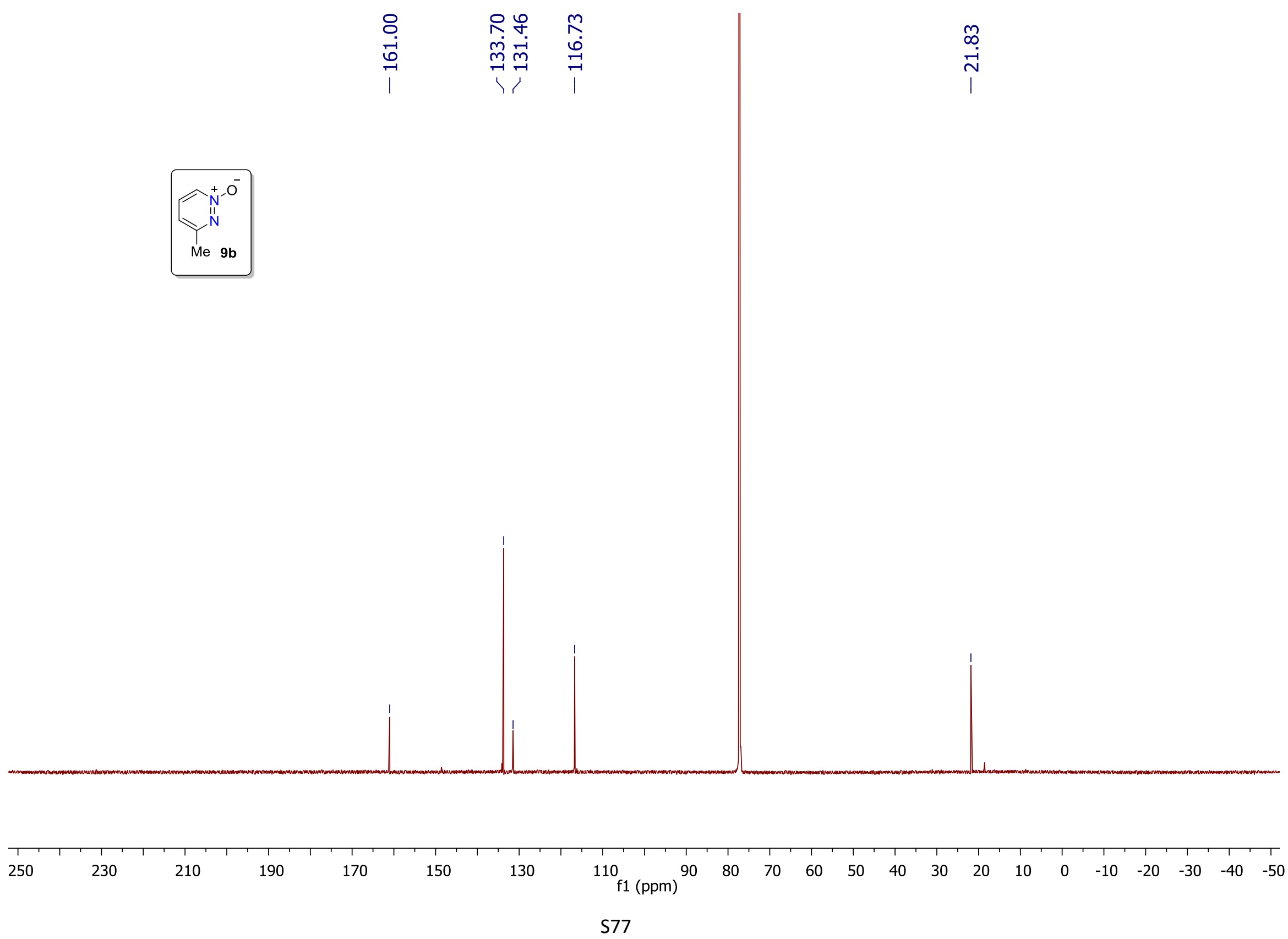

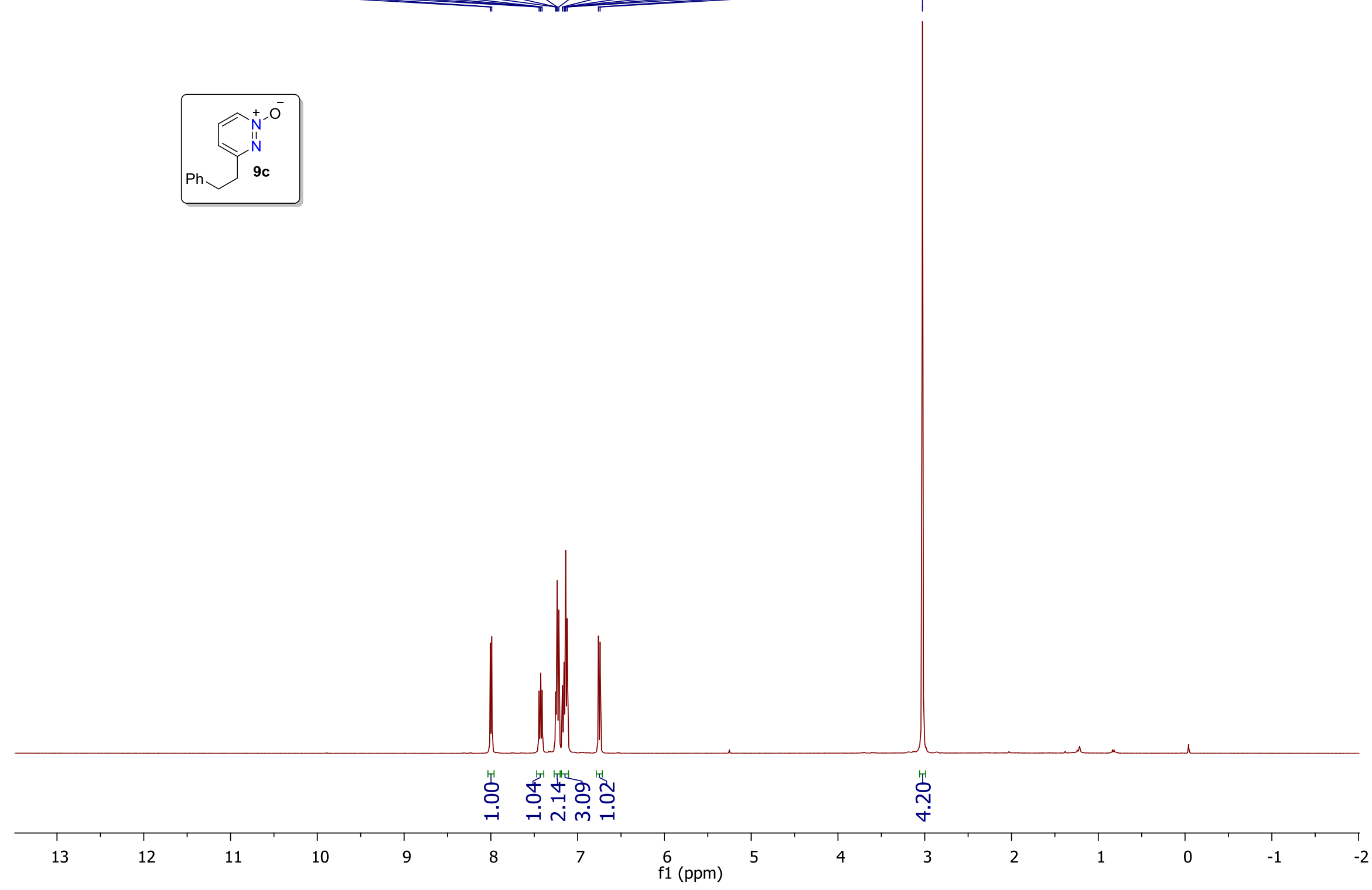

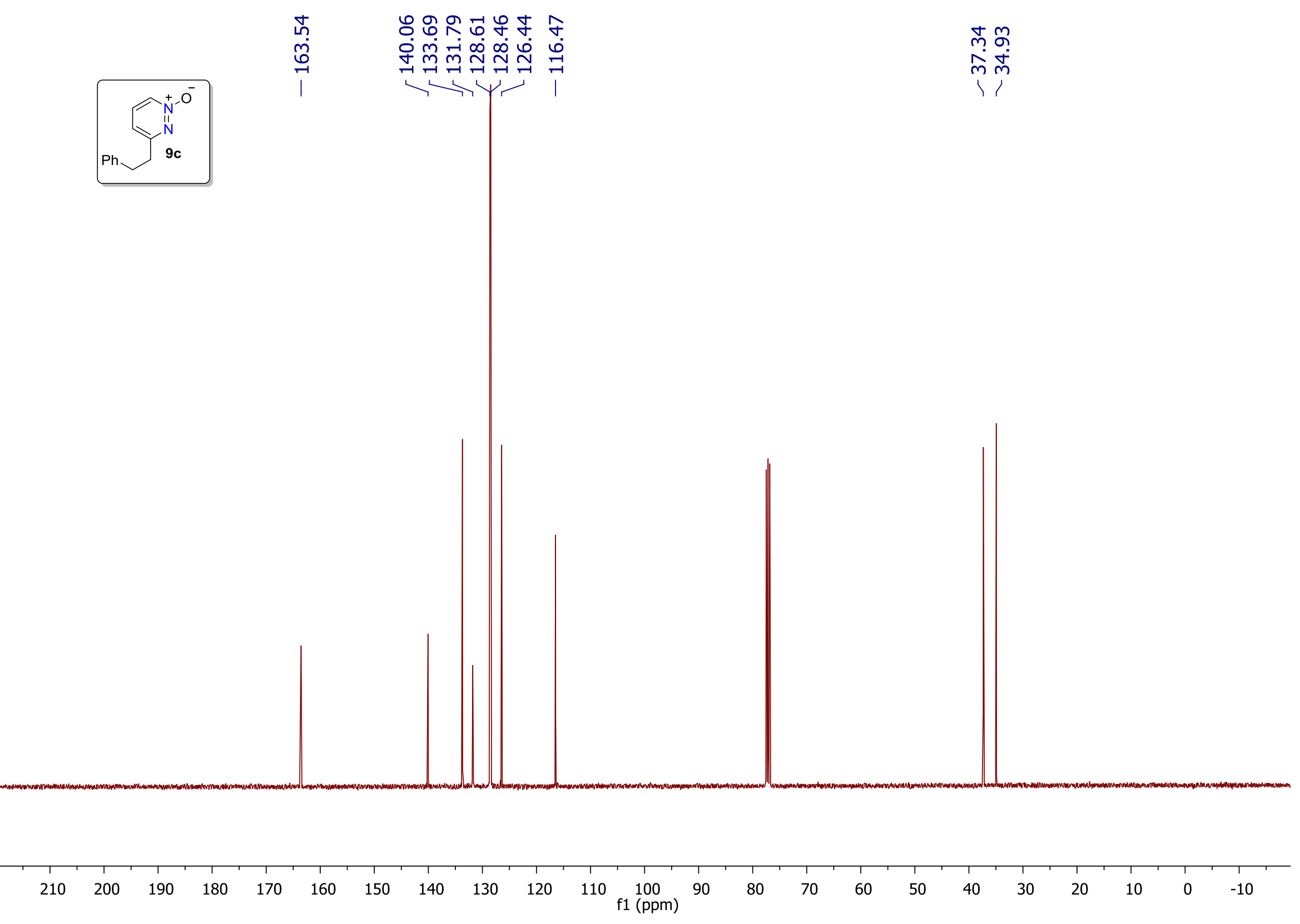
S79 
$\underbrace{\substack{0 \\ \infty}}_{\substack{n \\ \infty}}$

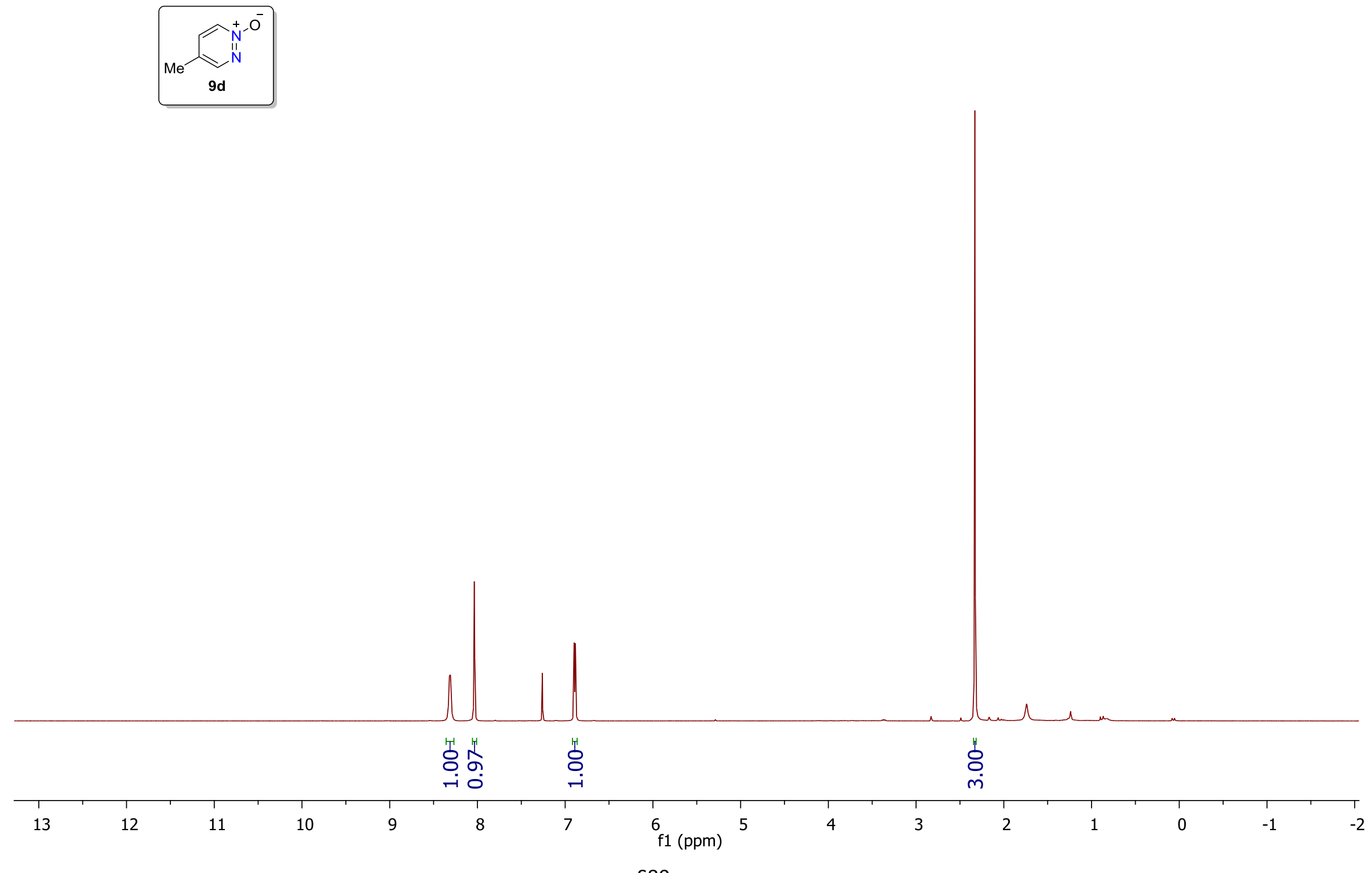

$\stackrel{m}{i}$ 


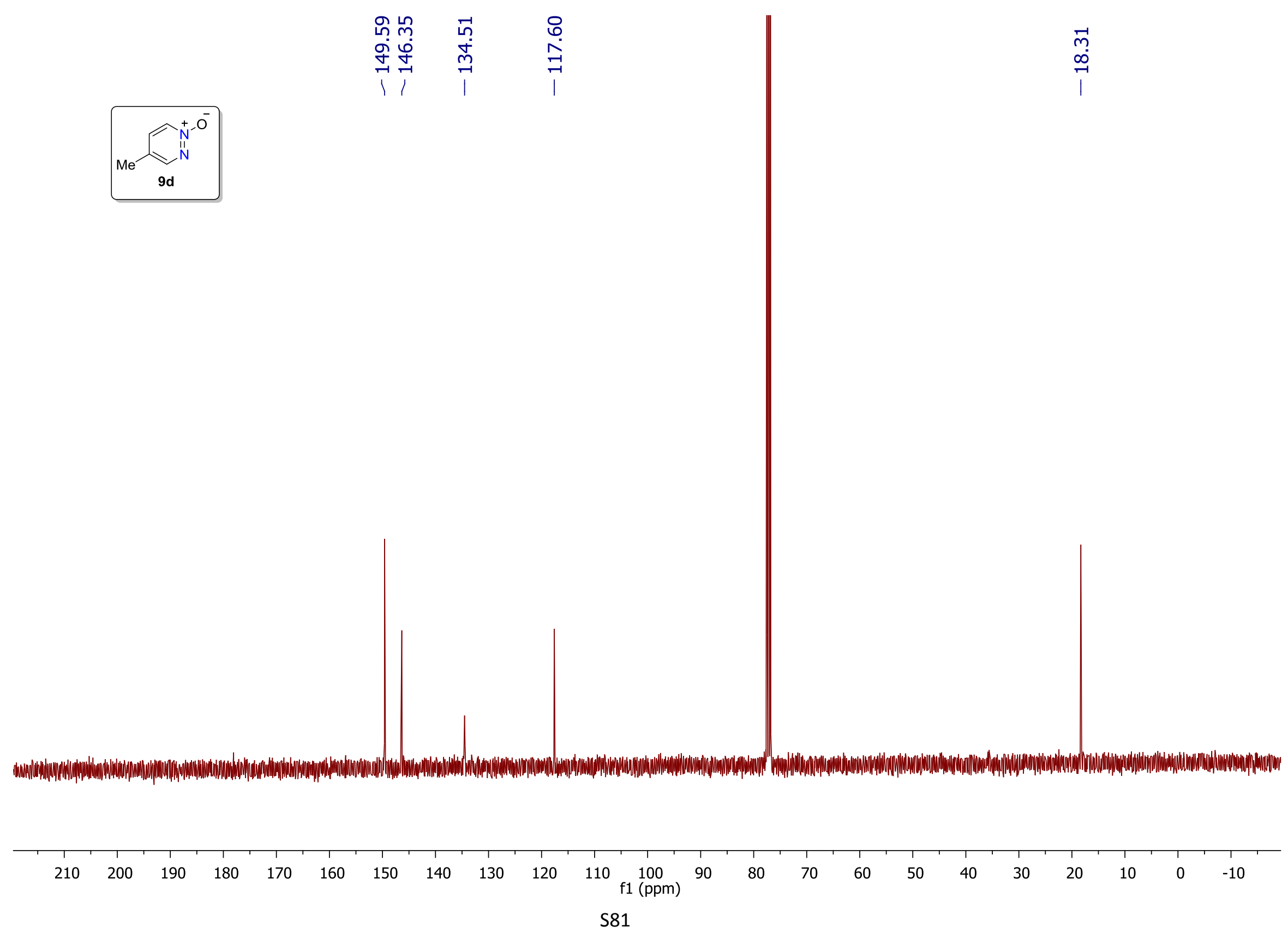




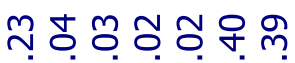

$\infty \infty \infty \infty \infty$

$\stackrel{\infty}{\sim} \underset{N}{N}$

ن

${ }_{9 e}^{M e} \underbrace{+O^{-}}_{\substack{N \\ N}}$

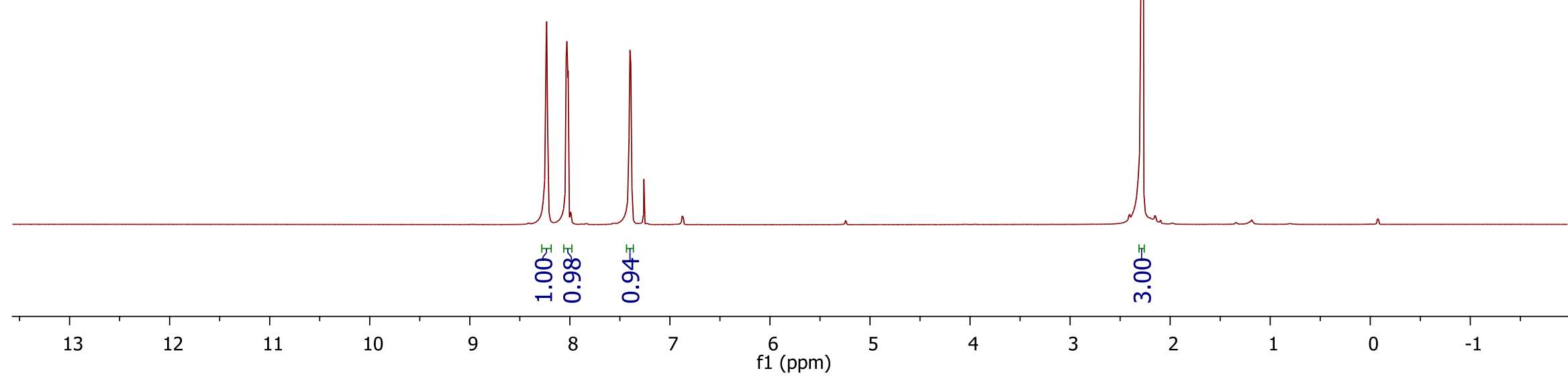



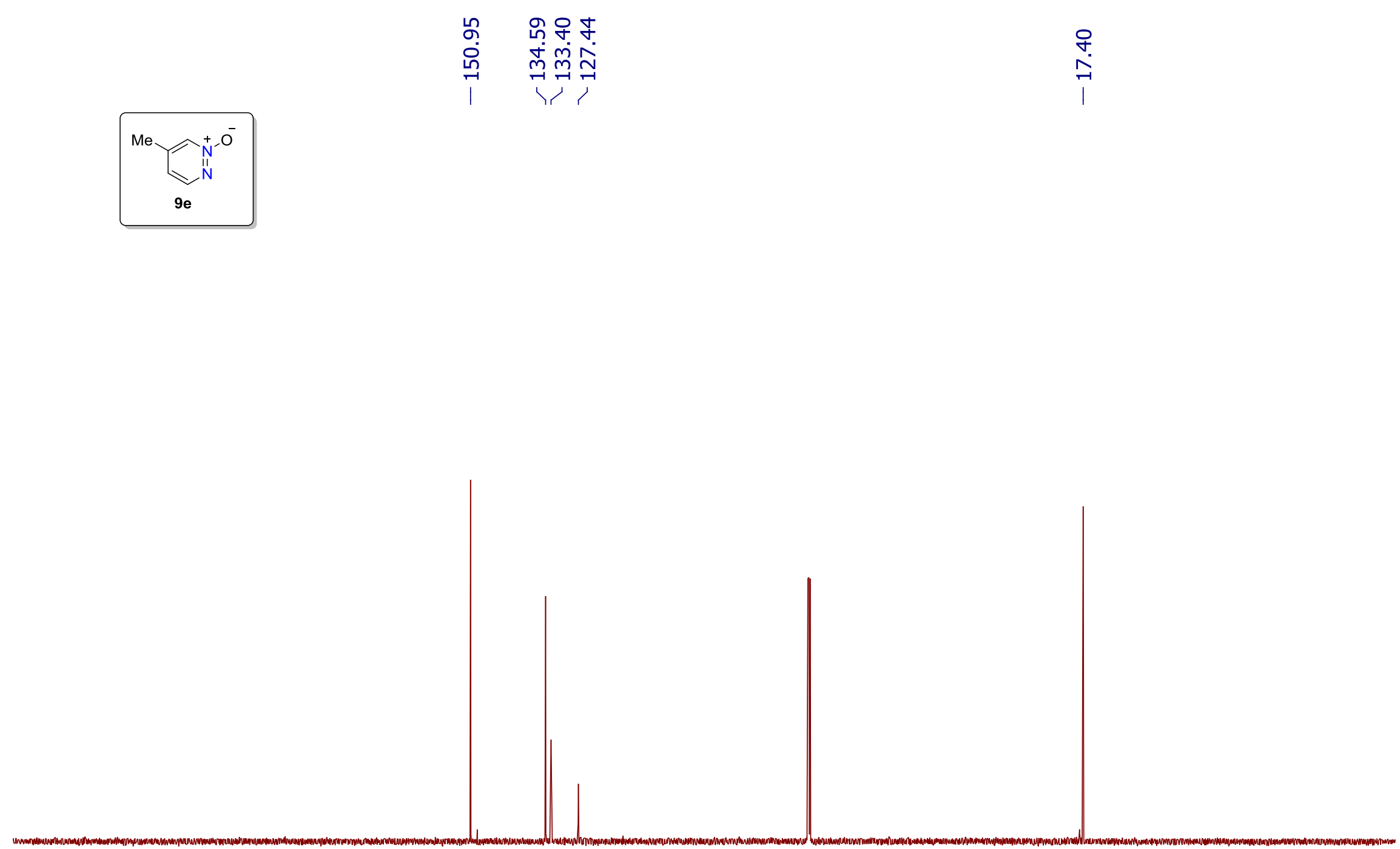

$\begin{array}{llllllllllllllllllllllllllllllllllll}250 & 240 & 230 & 220 & 210 & 200 & 190 & 180 & 170 & 160 & 150 & 140 & 130 & 120 & 110 & 100 & 90 & 80 & 70 & 60 & 50 & 40 & 30 & 20 & 10 & 0 & -10 & -20 & -30 & -40 & -50\end{array}$ f1 (ppm) 


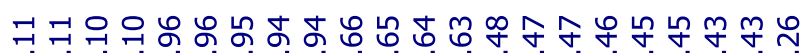

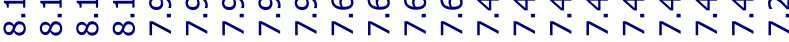
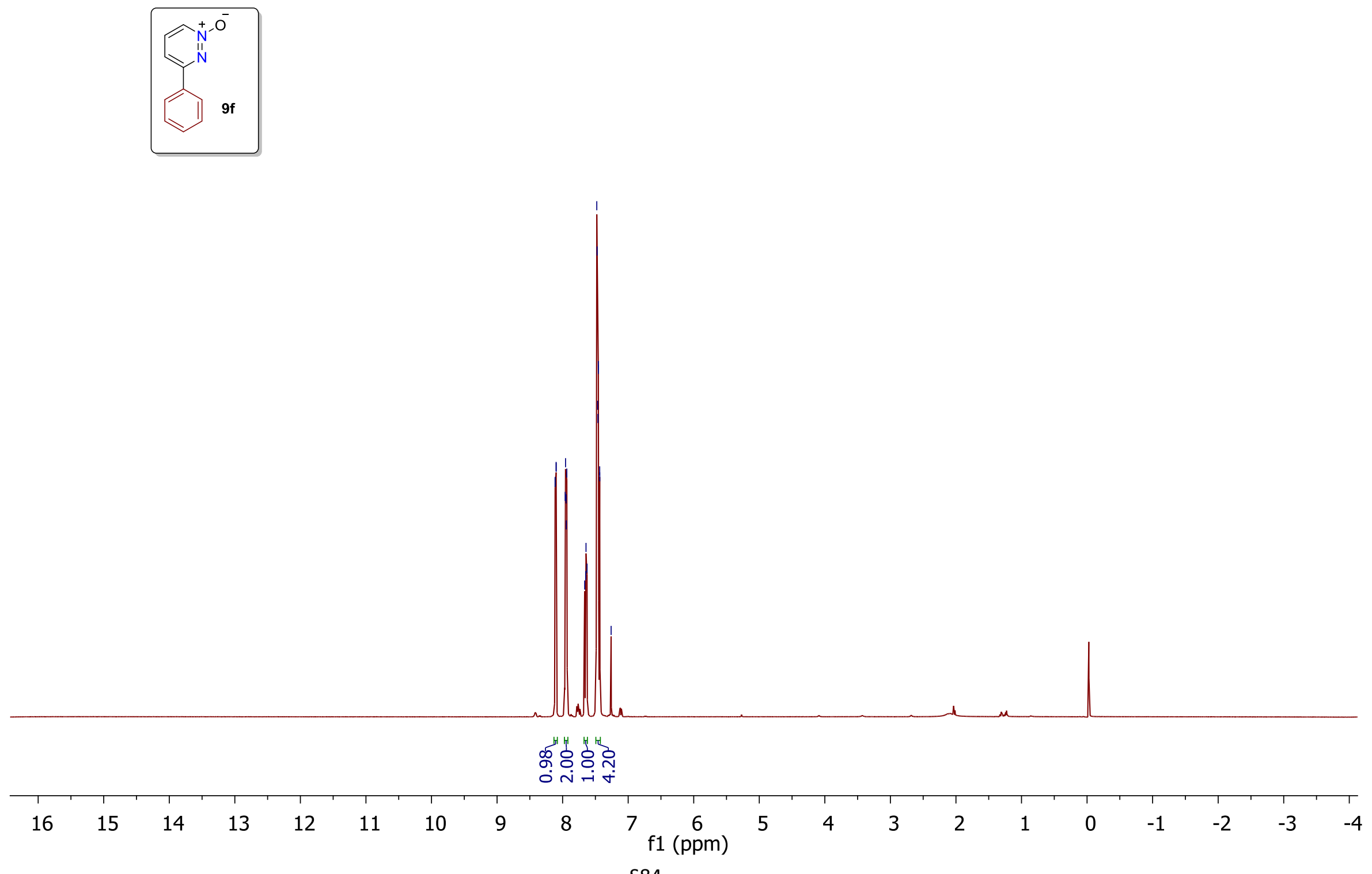

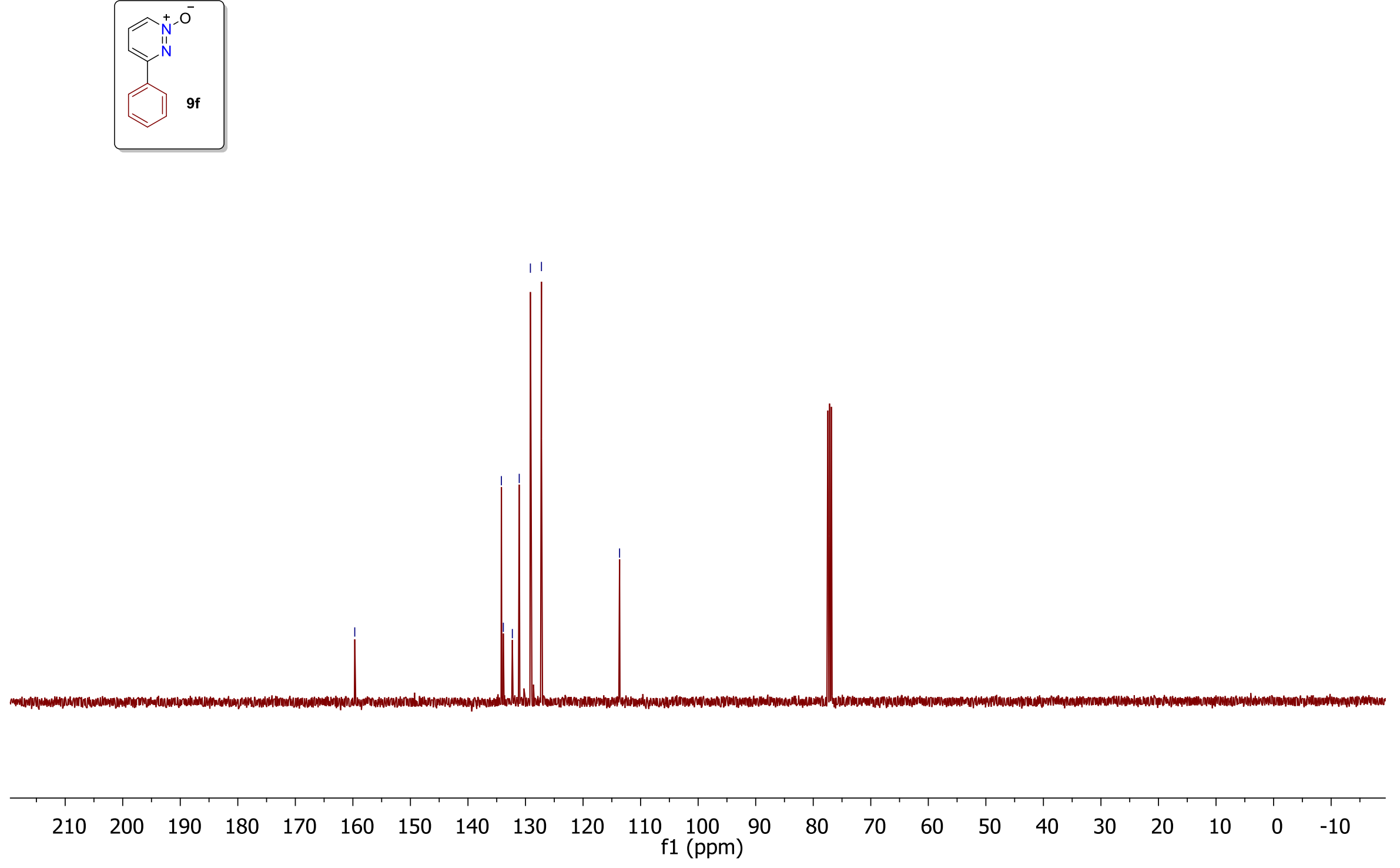
車
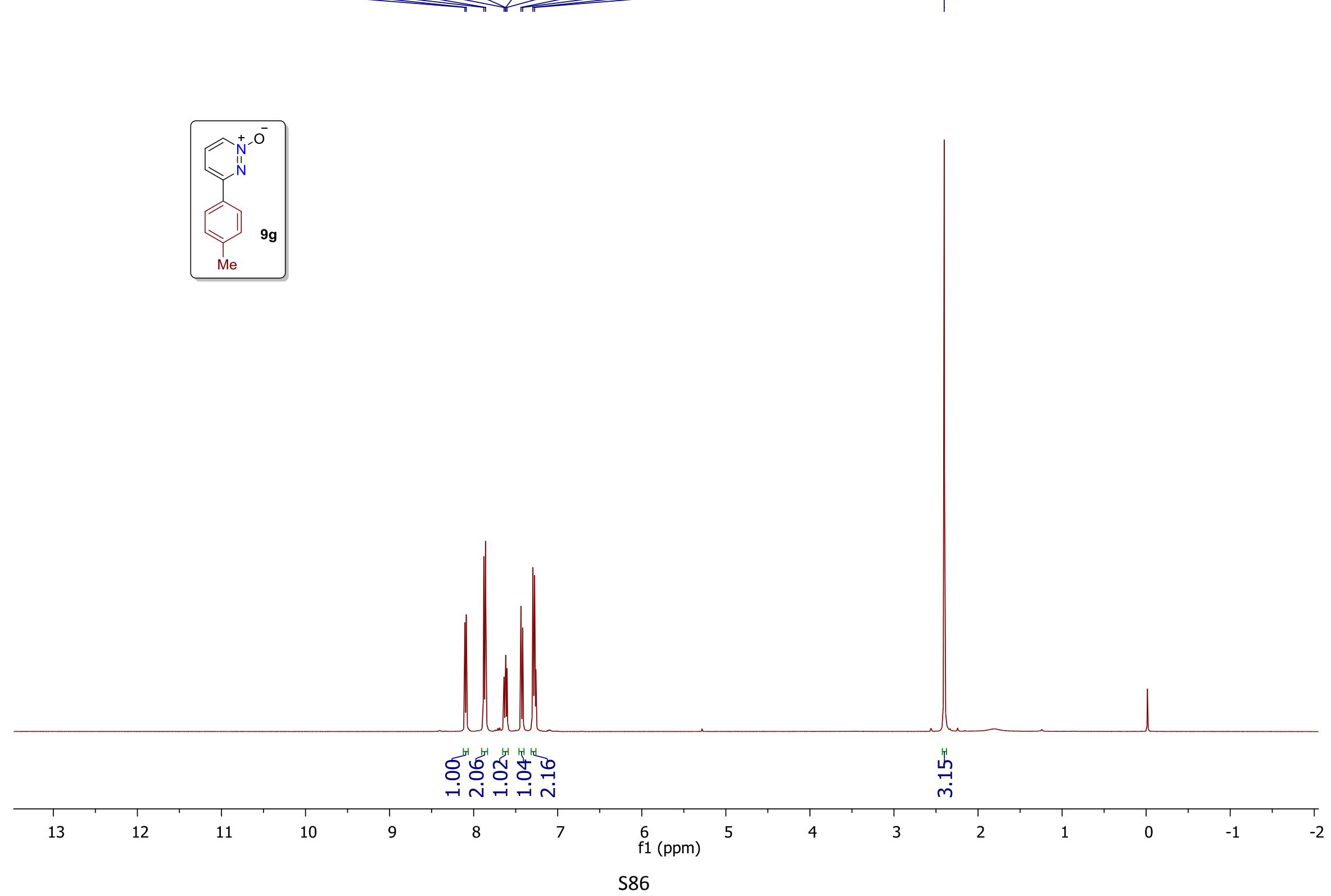


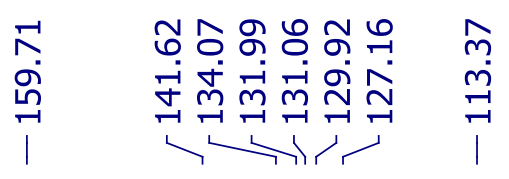
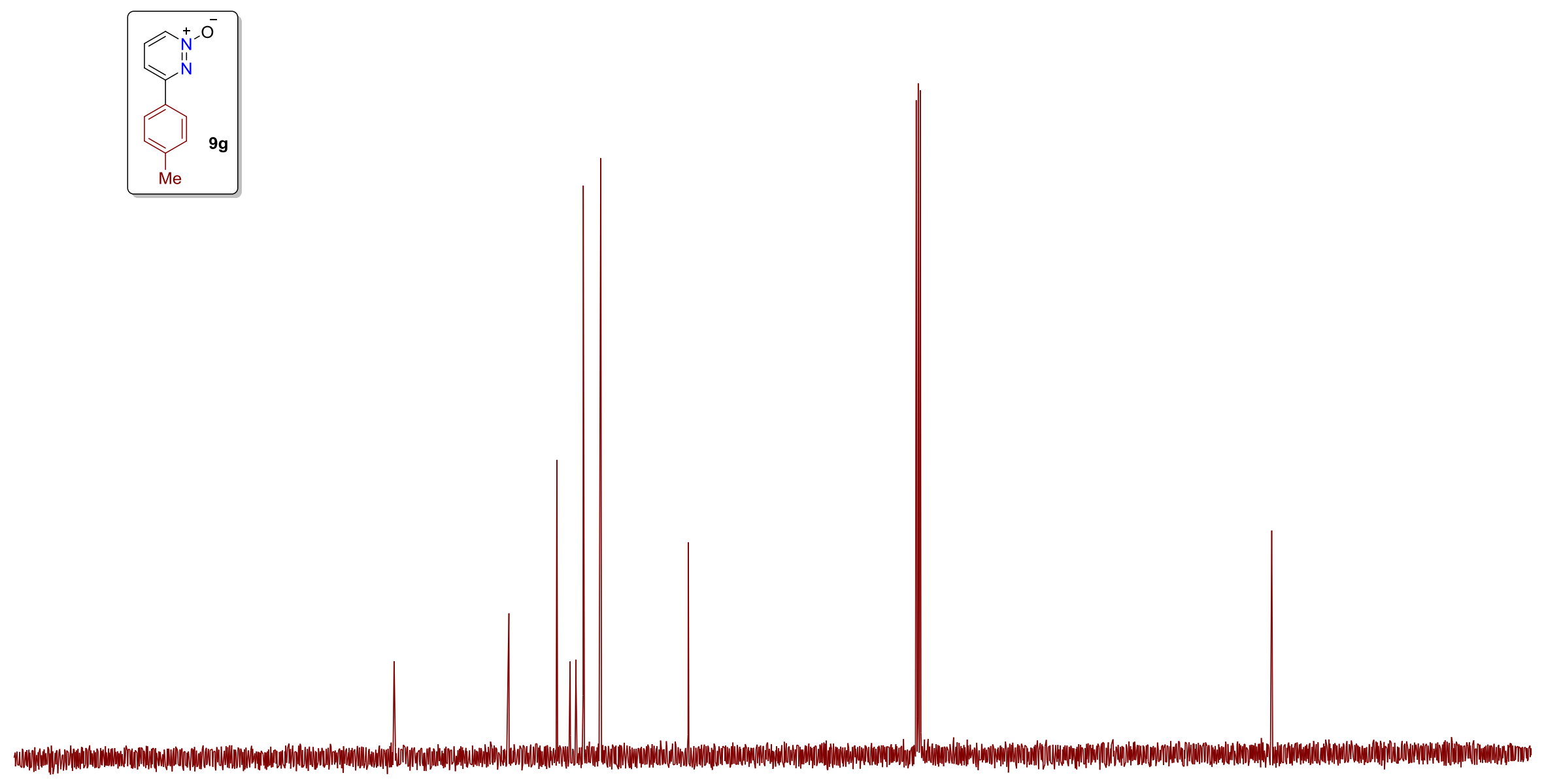

$\begin{array}{lllllllll}210 & 200 & 190 & 180 & 170 & 160 & 150 & 140 & 130\end{array}$ 


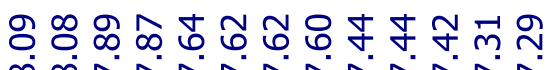

$\infty \infty^{\circ} N \hat{N} N \hat{N} N \hat{N}$
Nㅜㅇ유유유ำ

nin

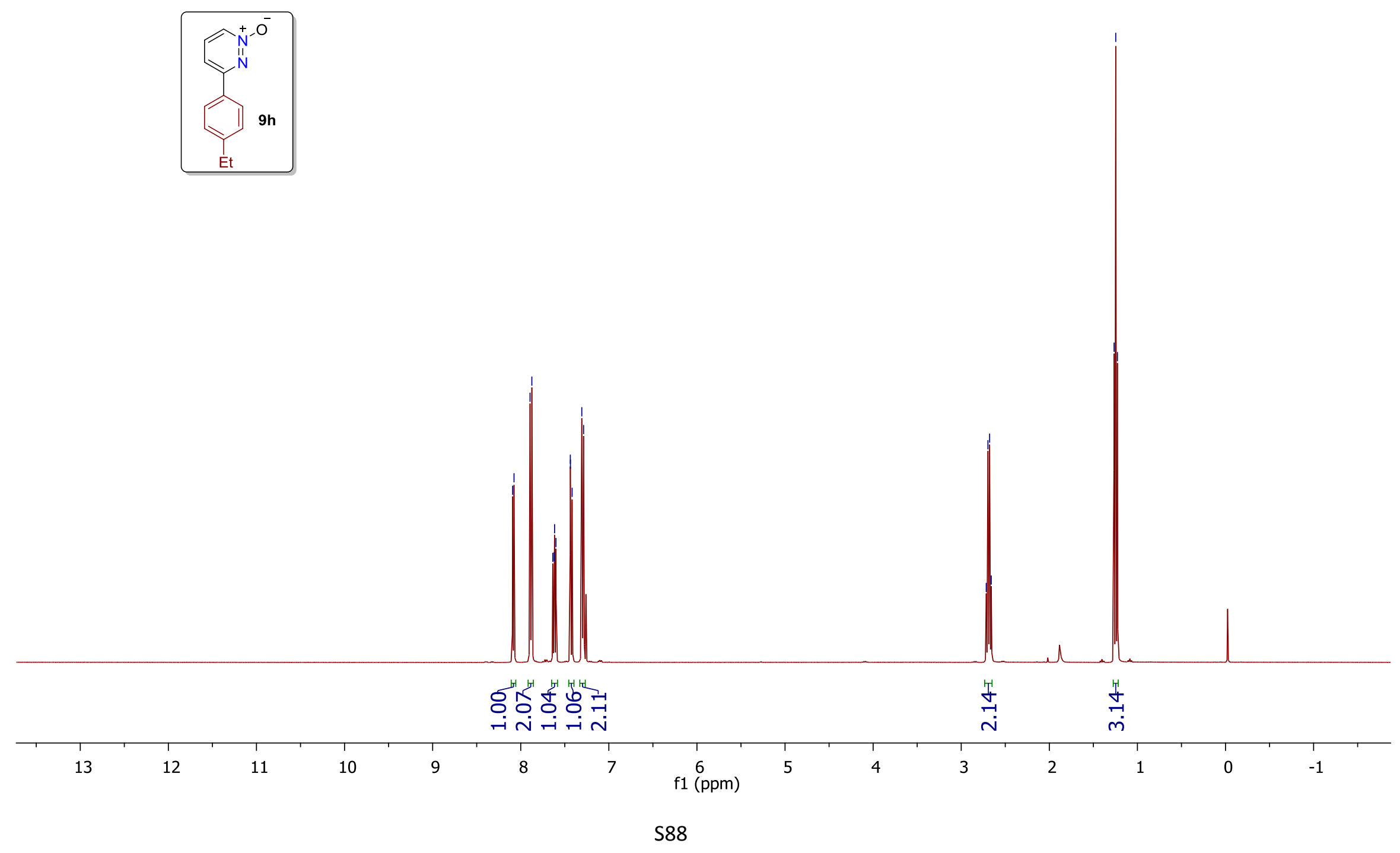



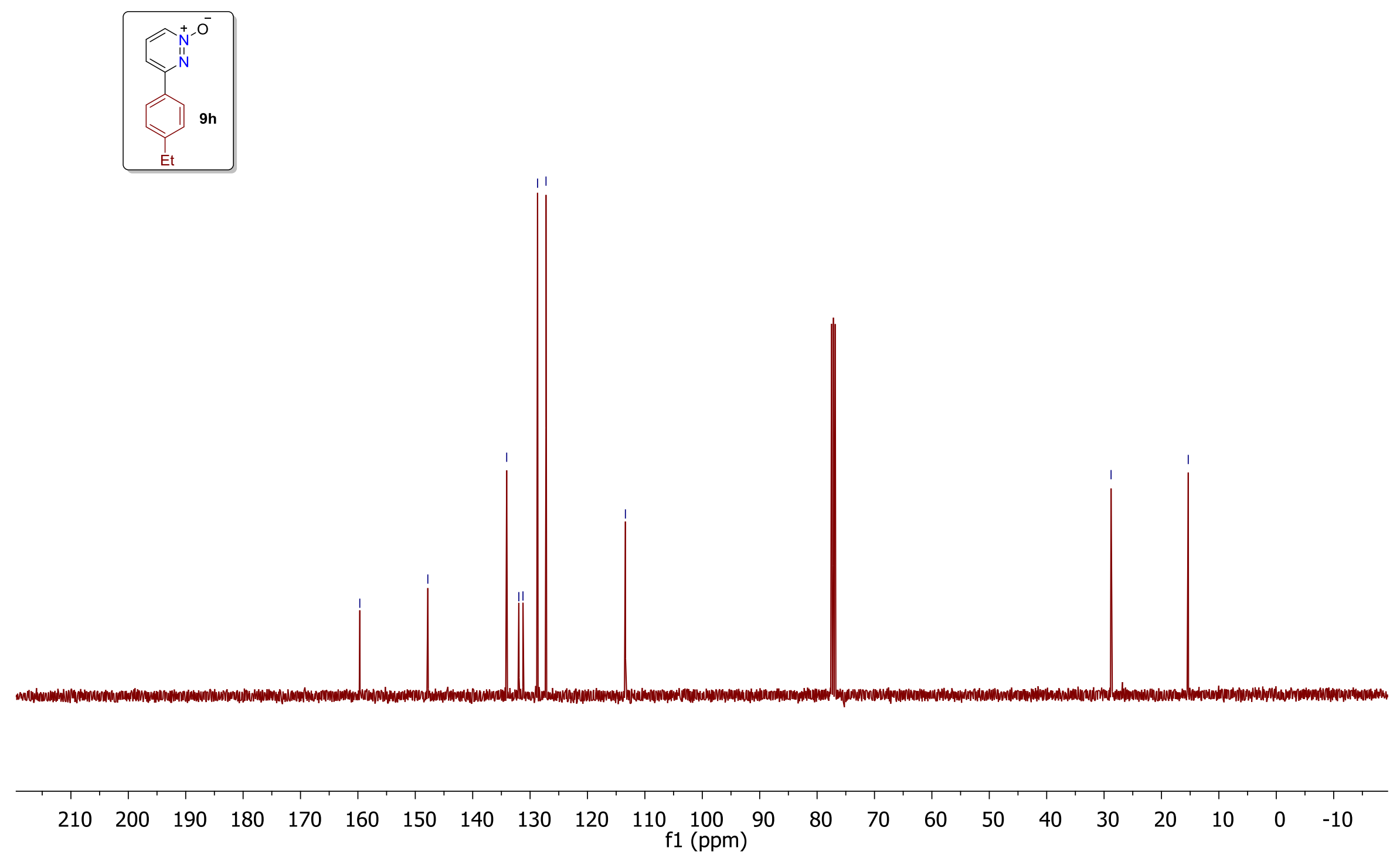


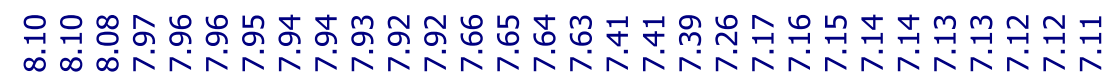

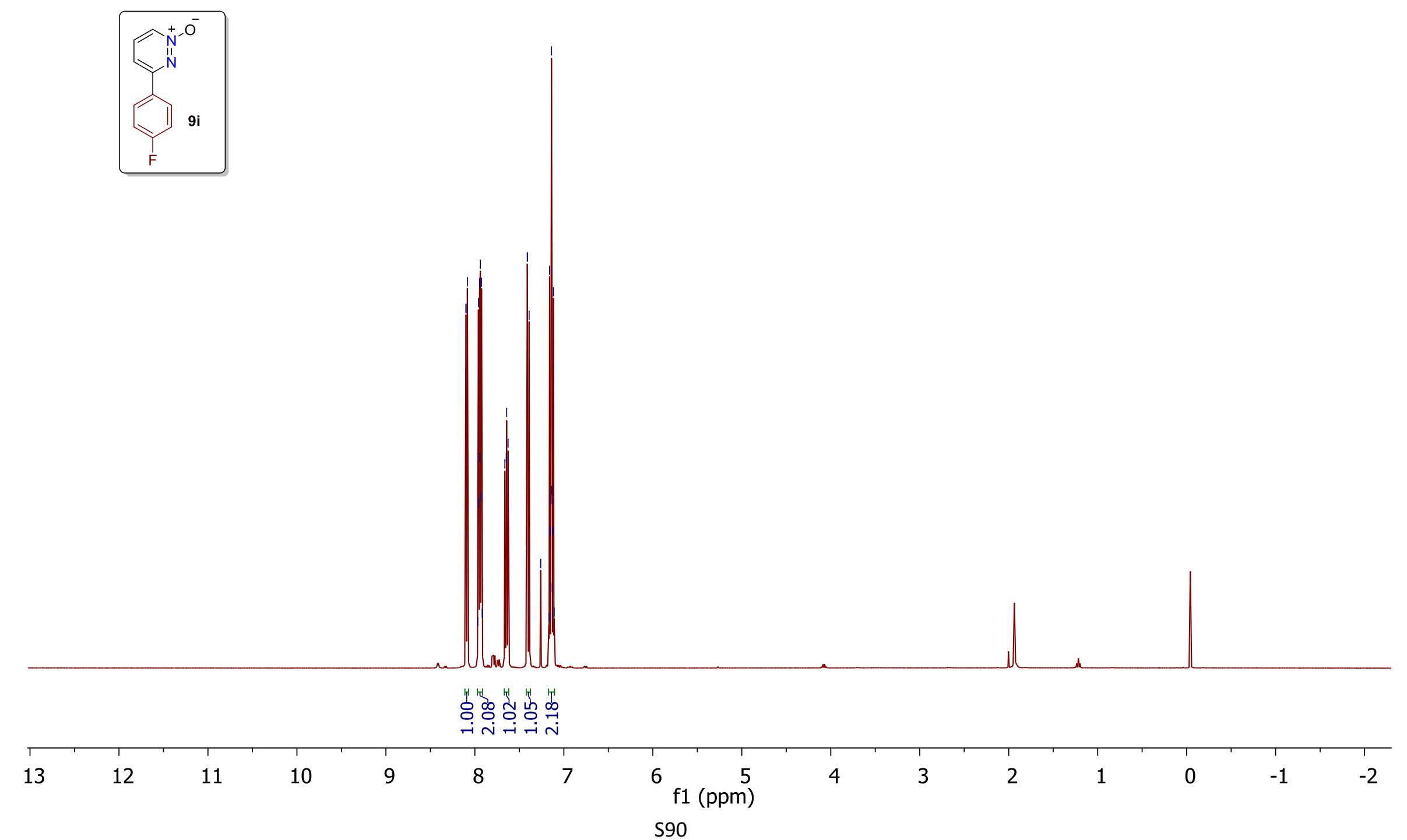




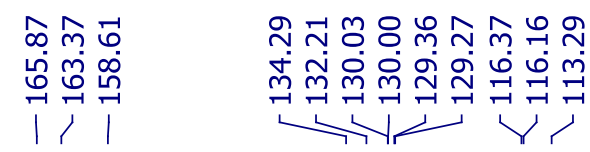
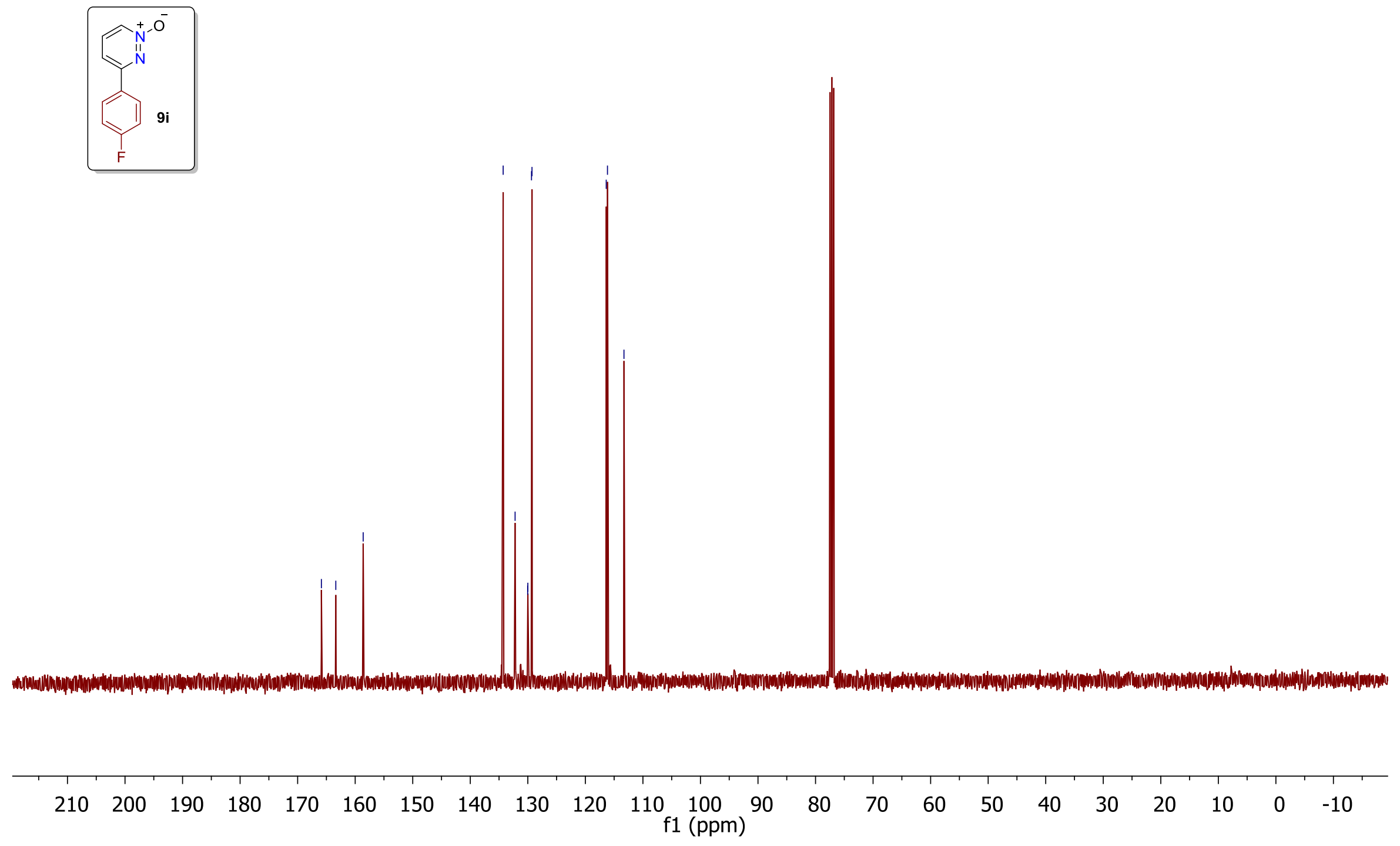


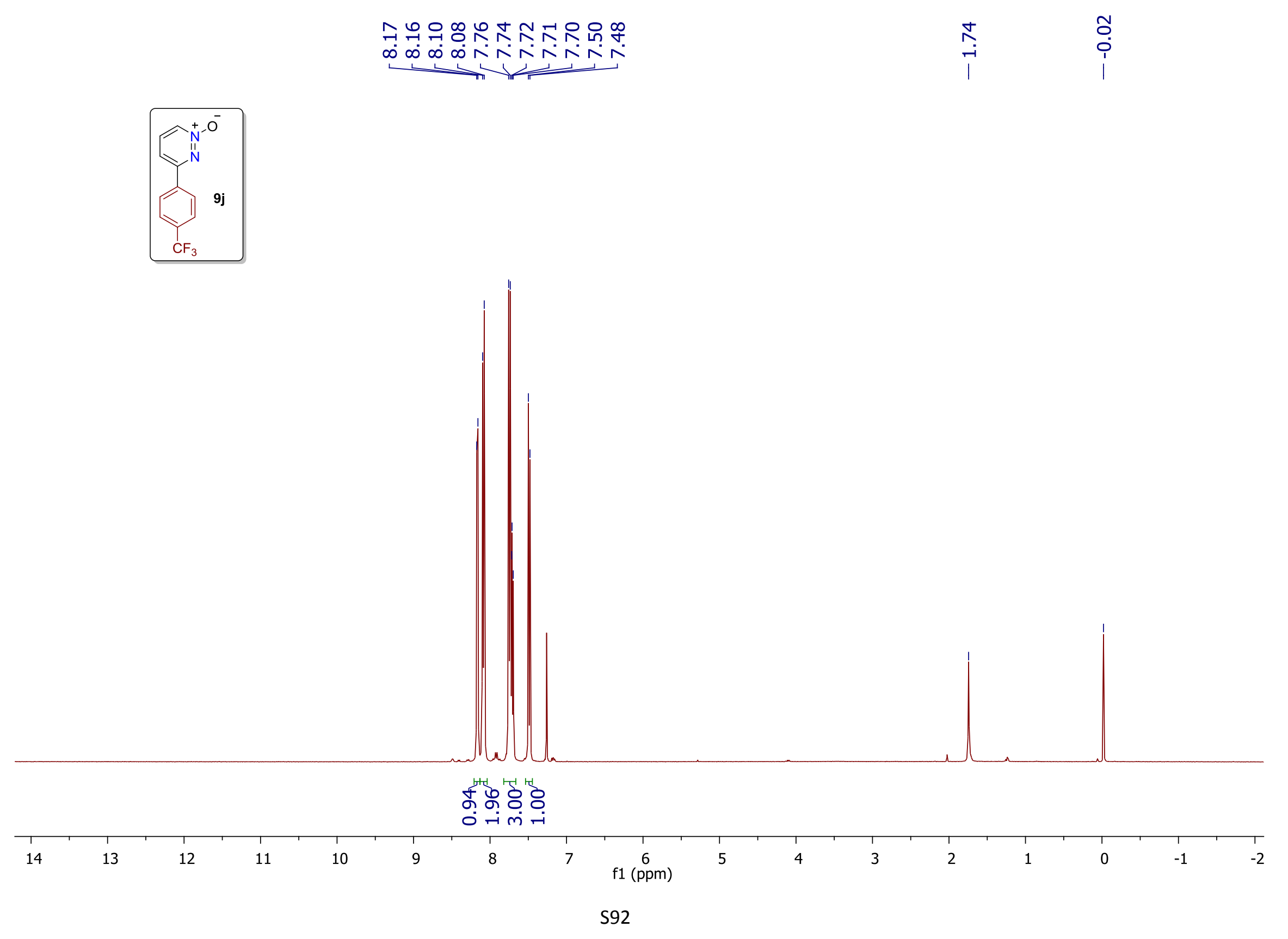



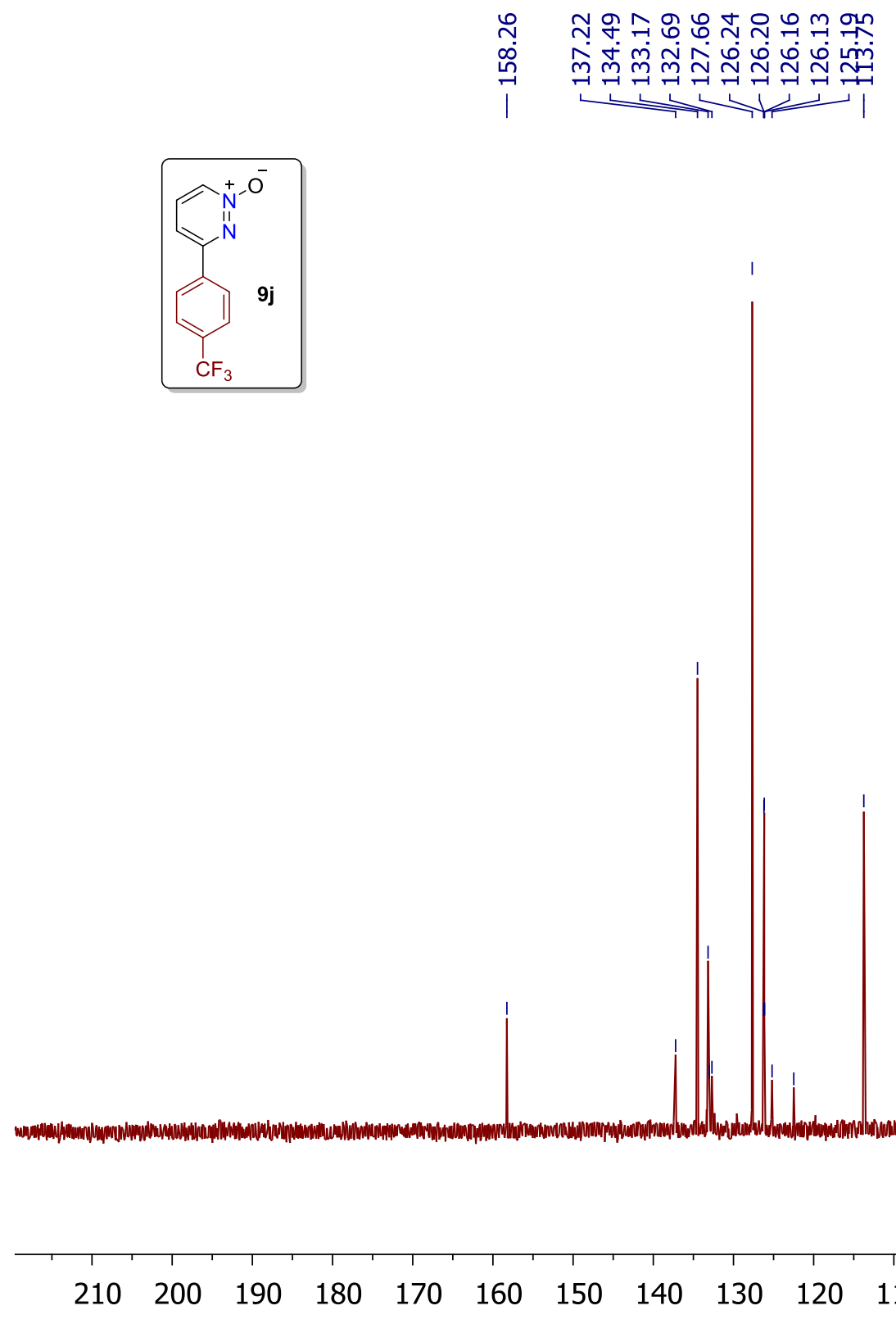
ํํํํำ

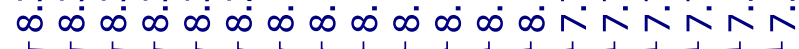
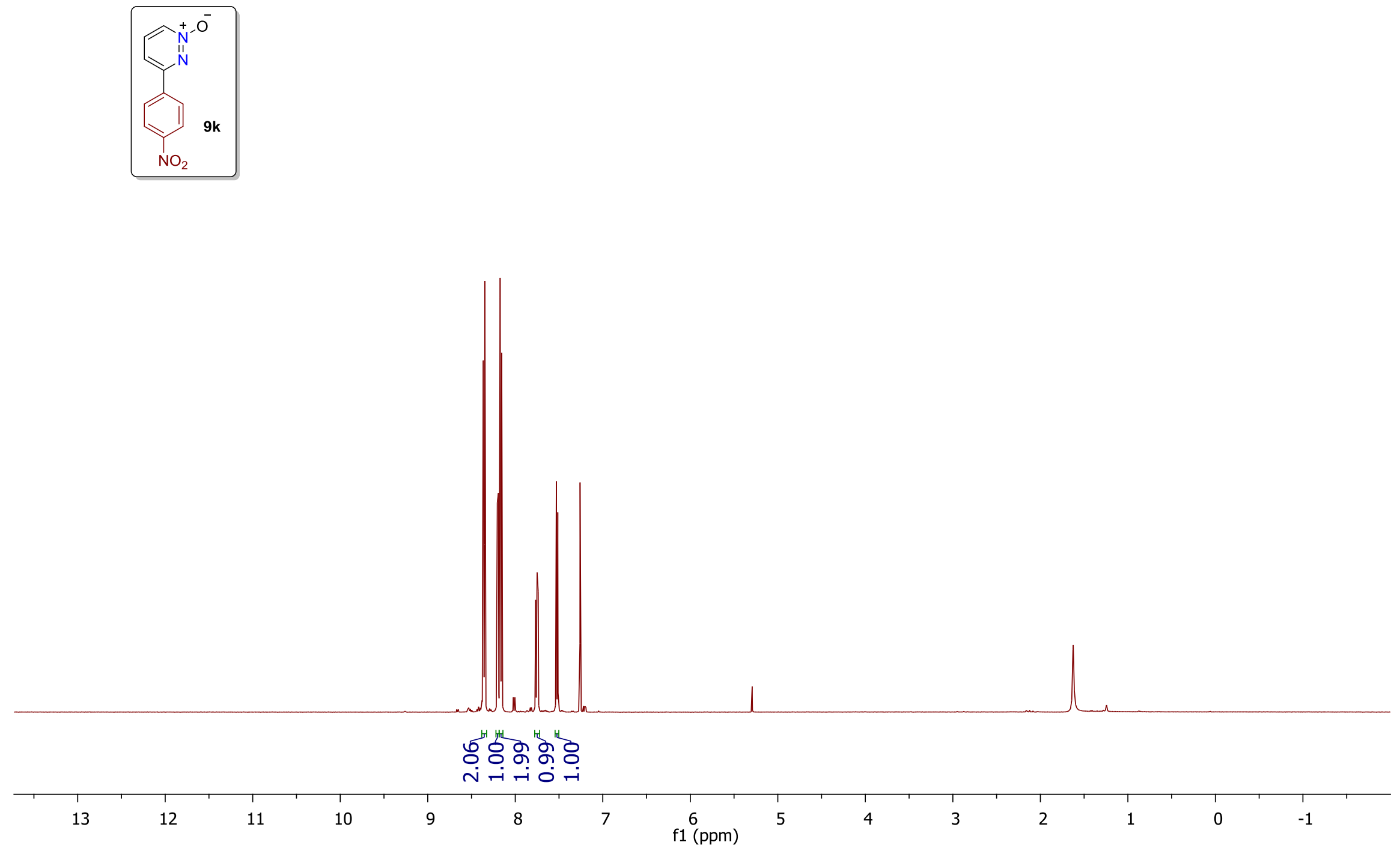

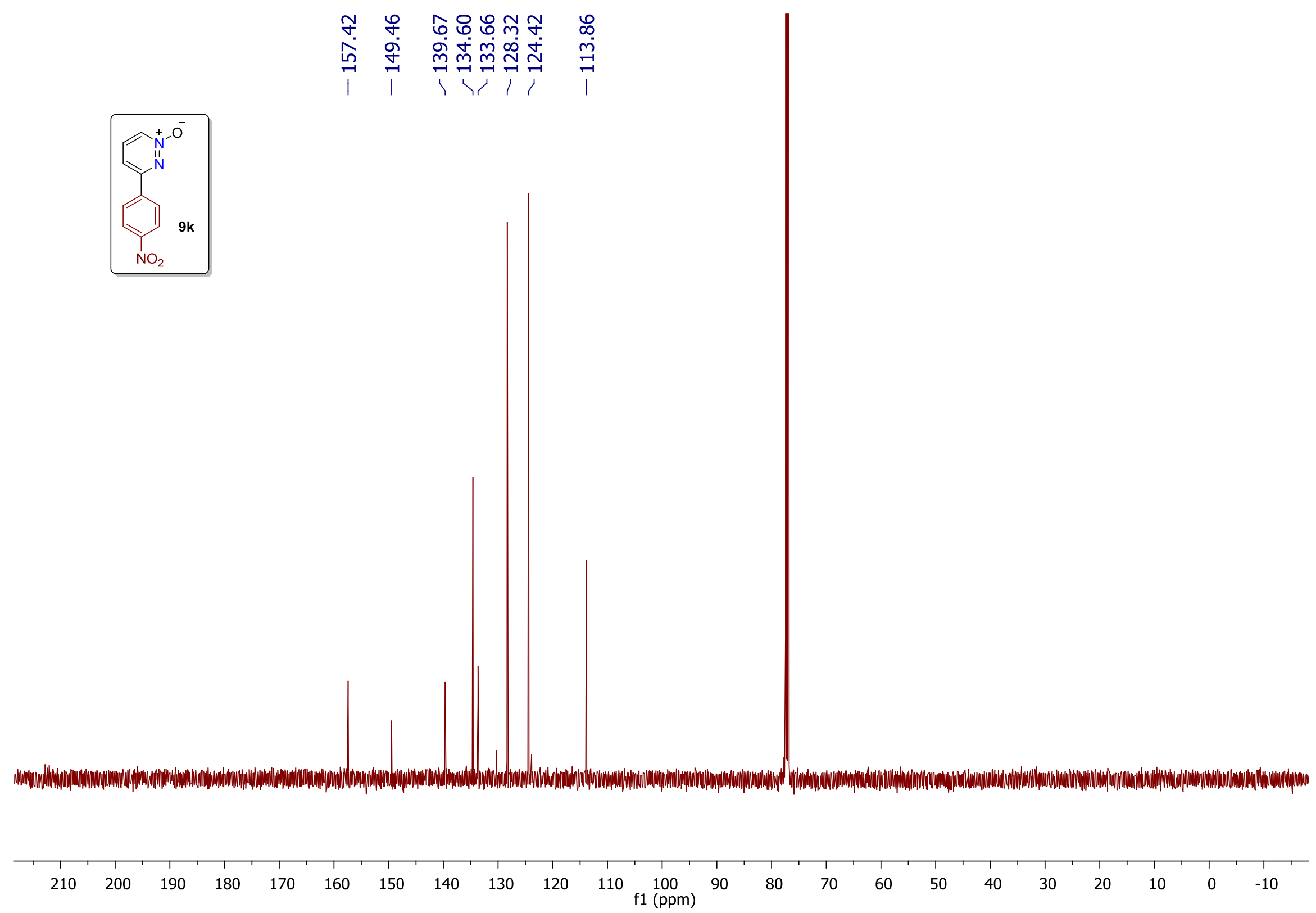


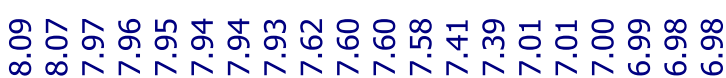

$\hat{\infty}$

$m^{m}$
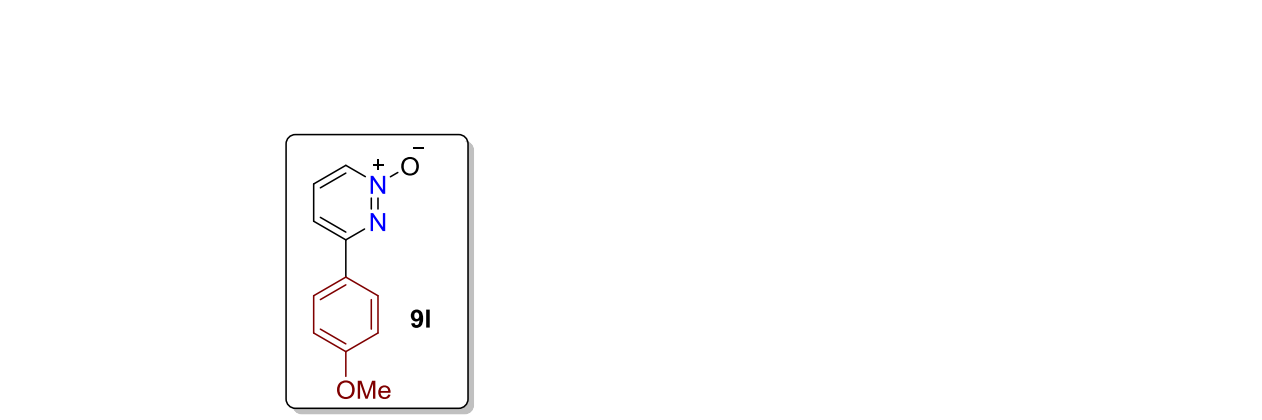


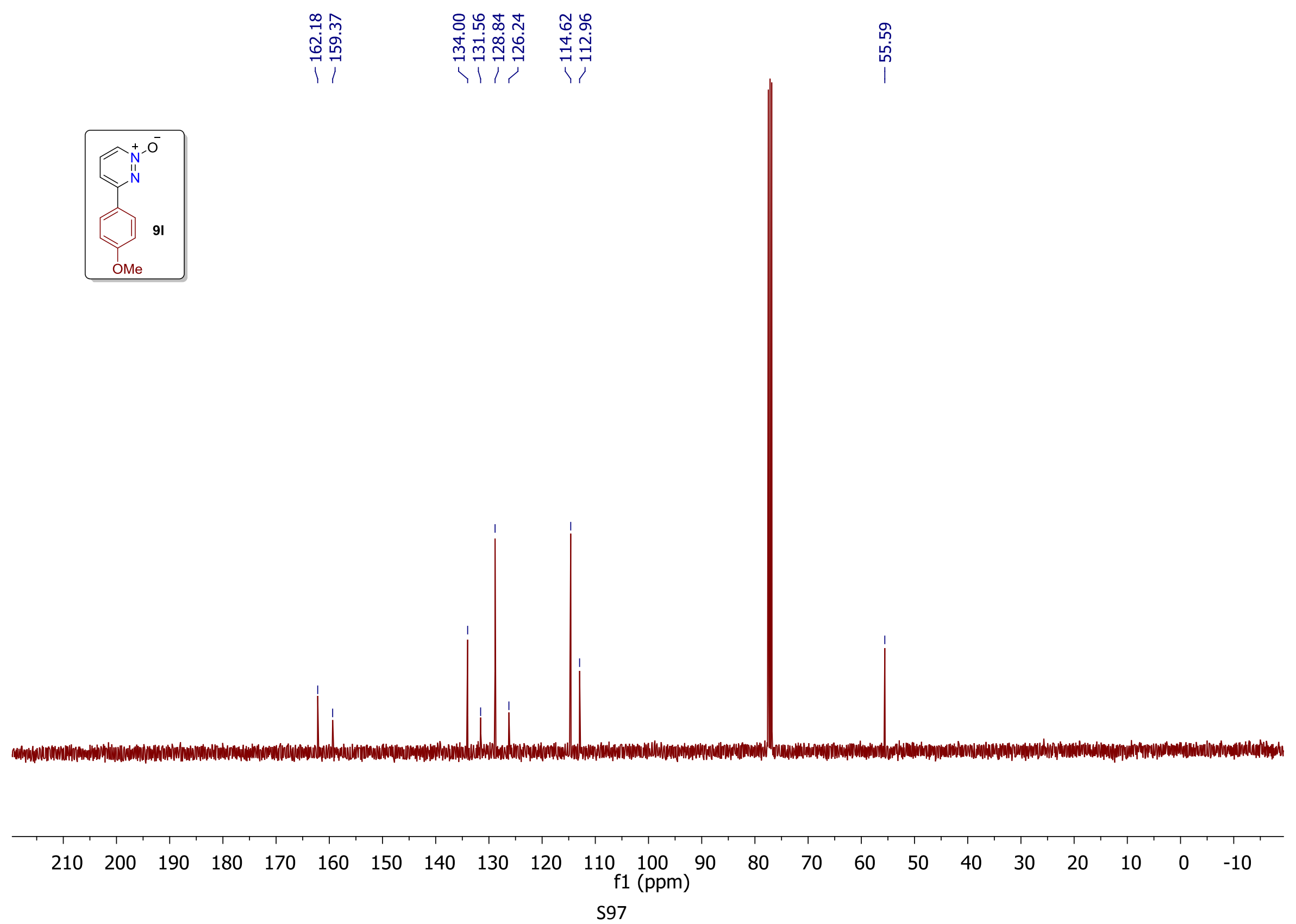




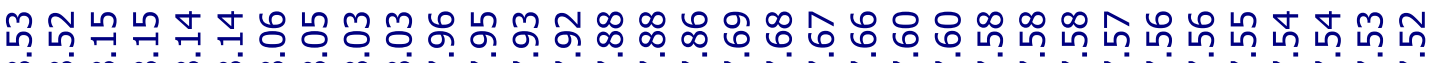

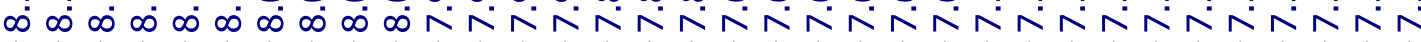
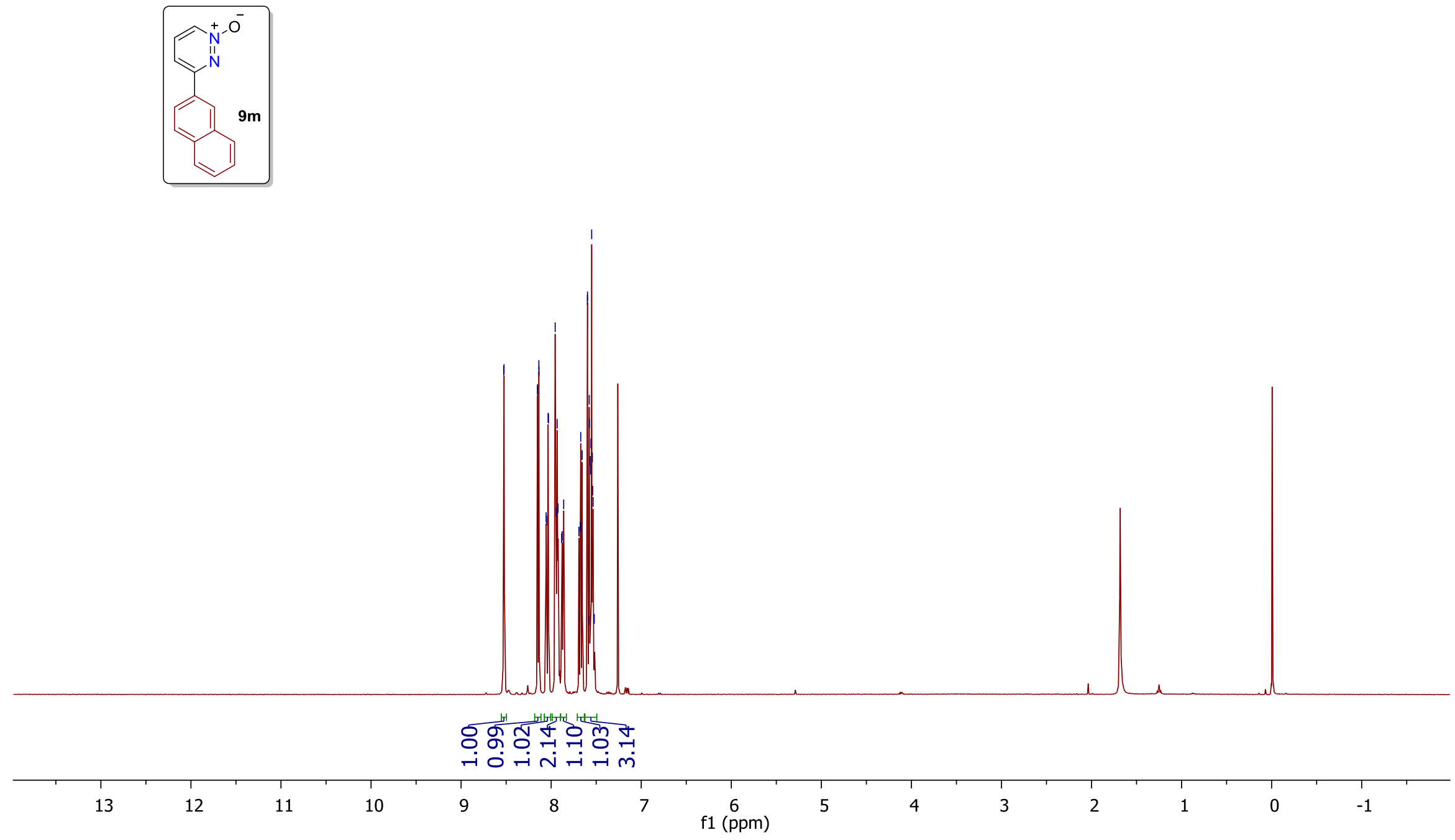


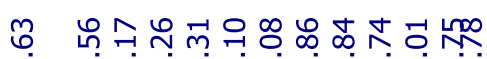

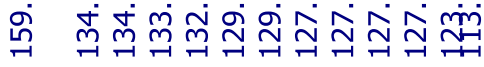
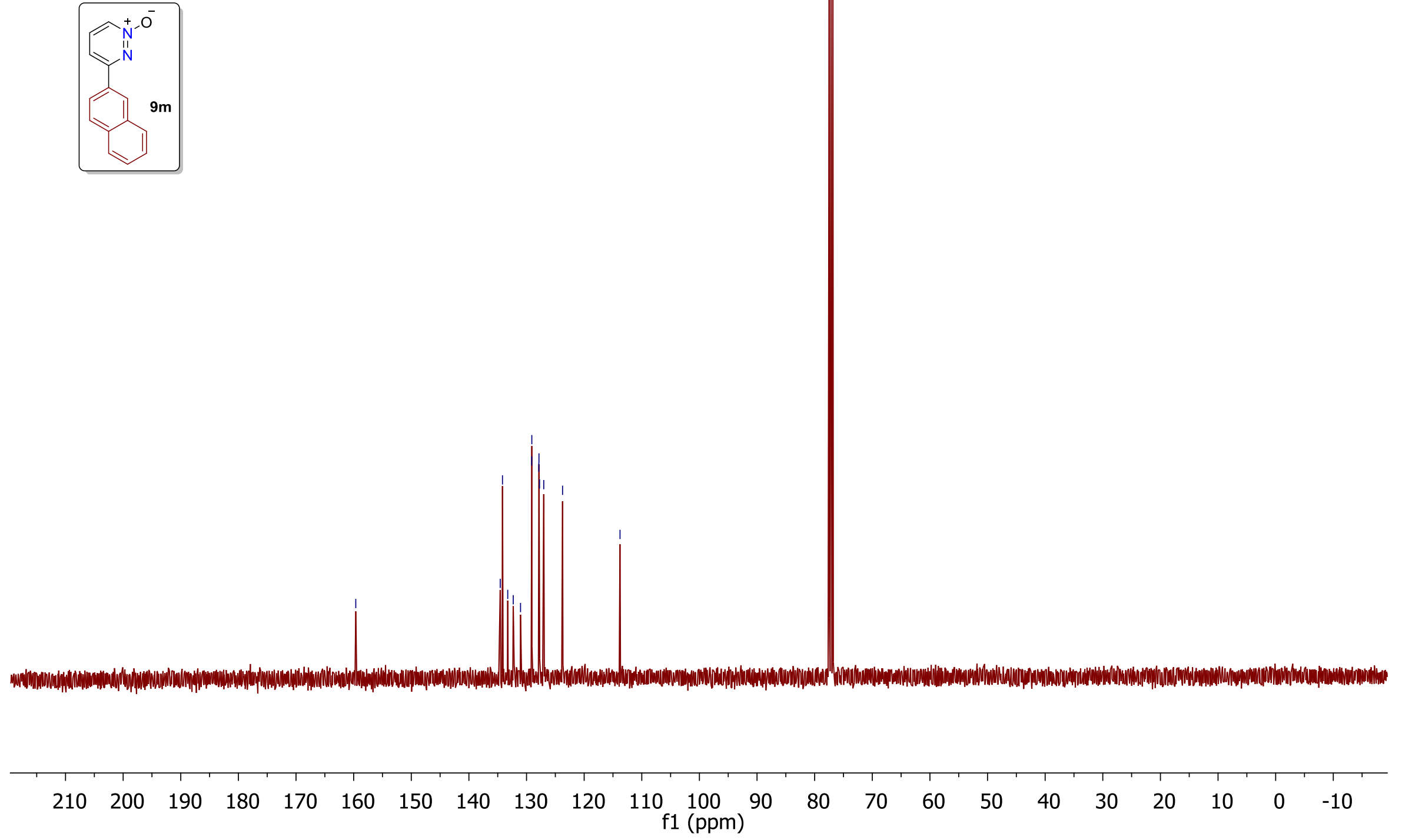


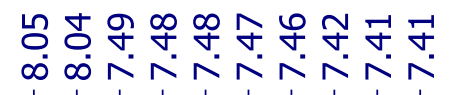

$\stackrel{\infty}{\sim}$

$\stackrel{\sim}{\text { I }}$
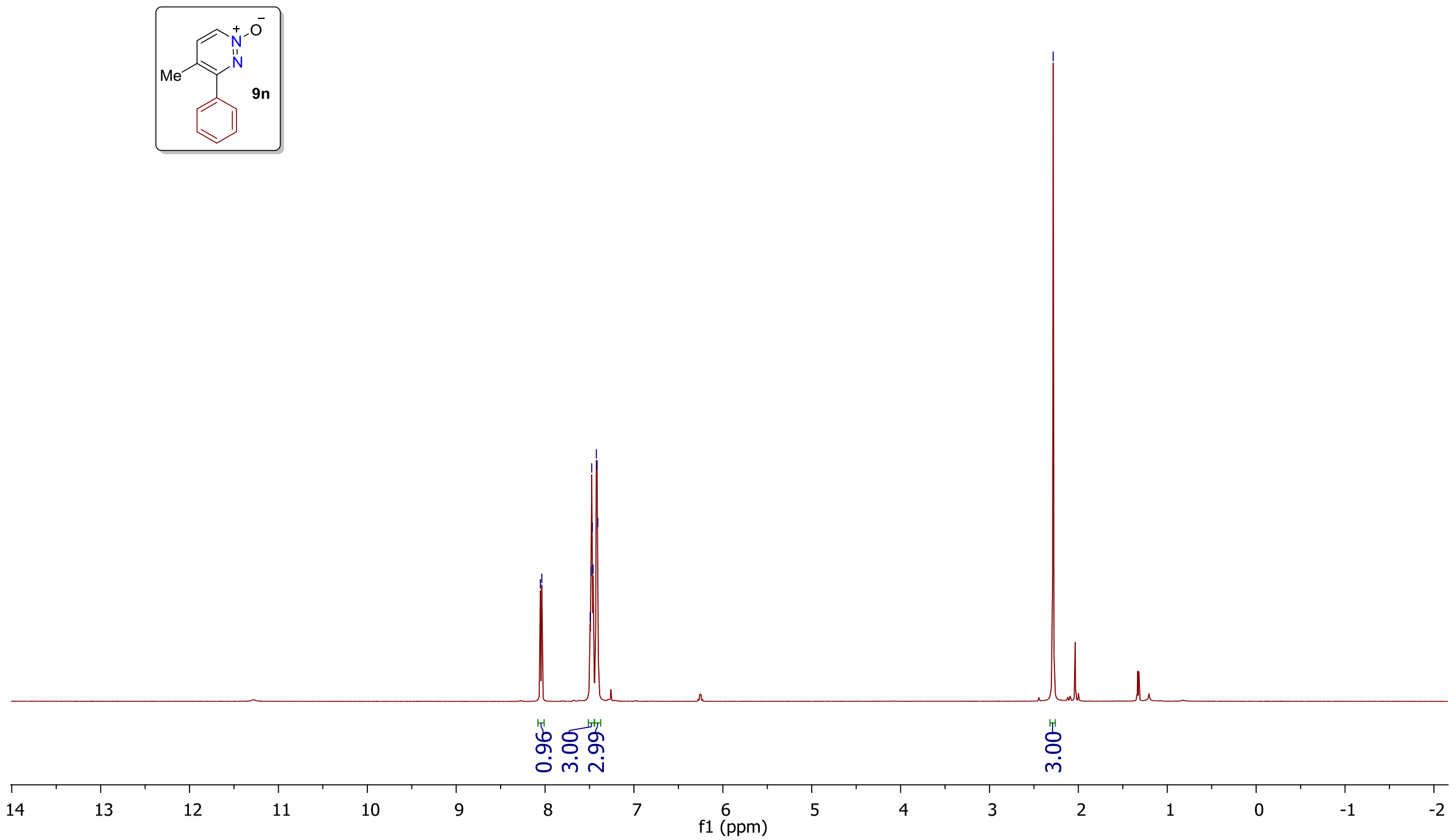

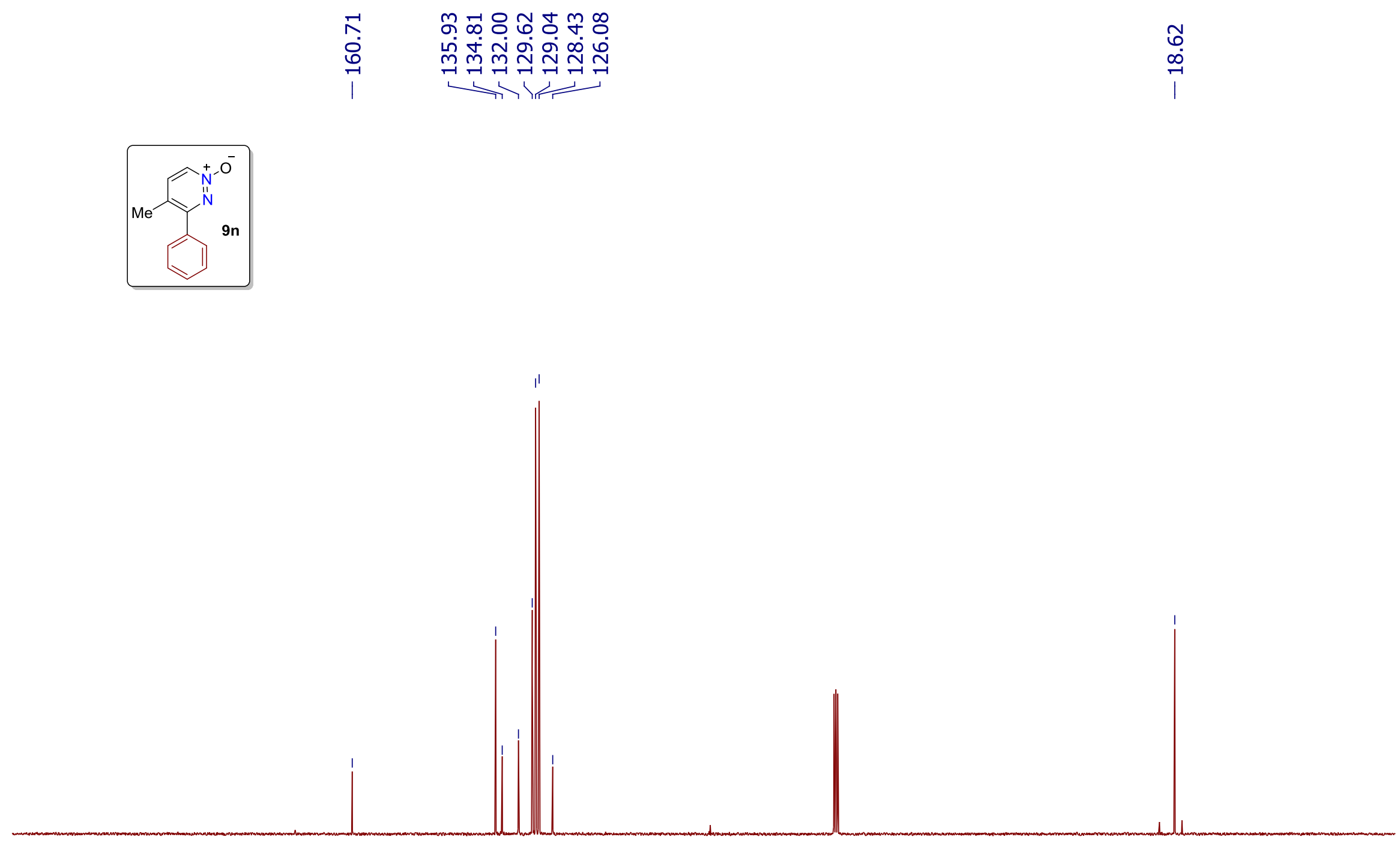


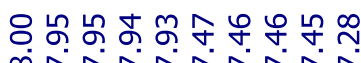

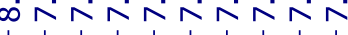

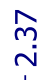

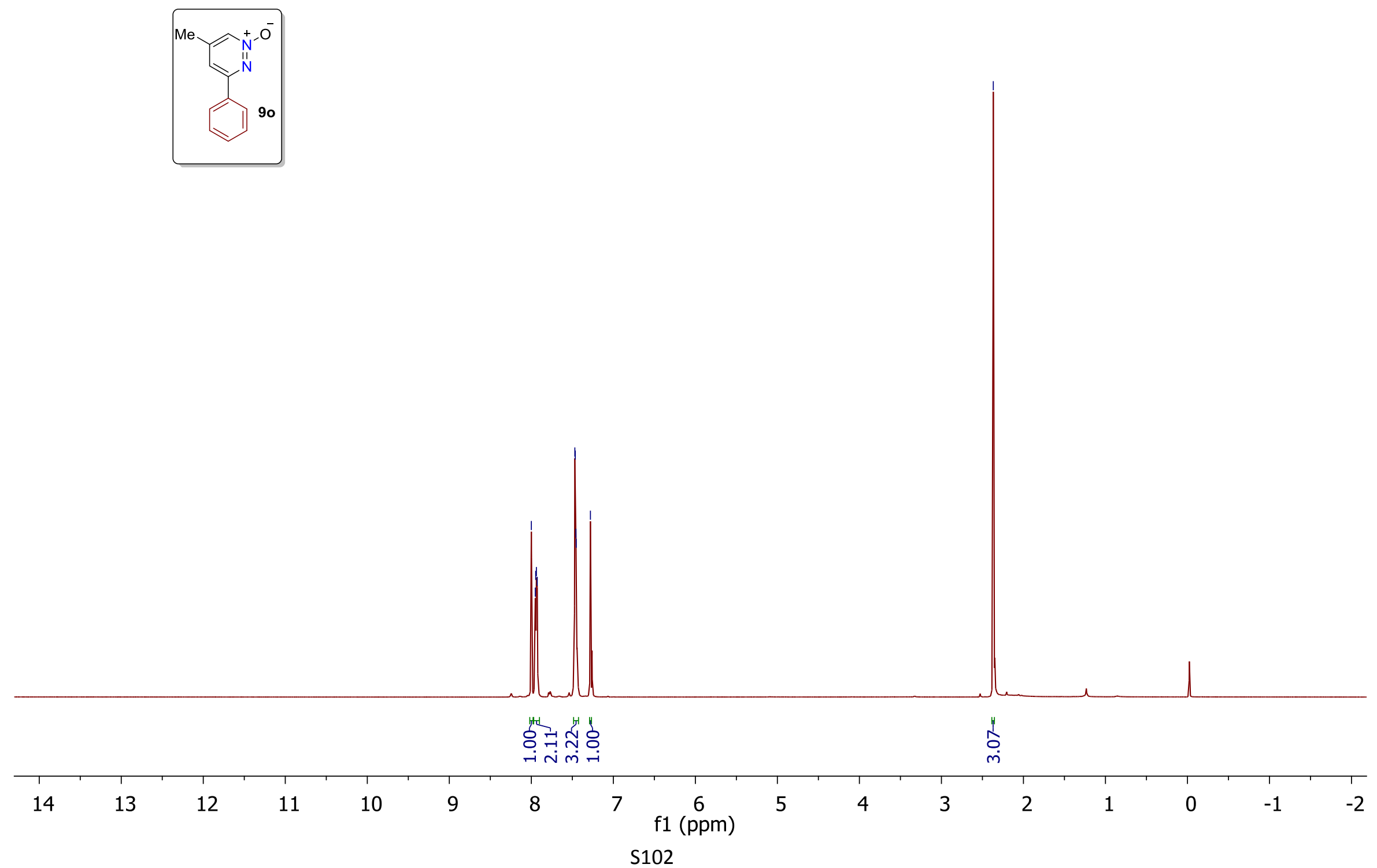

i 

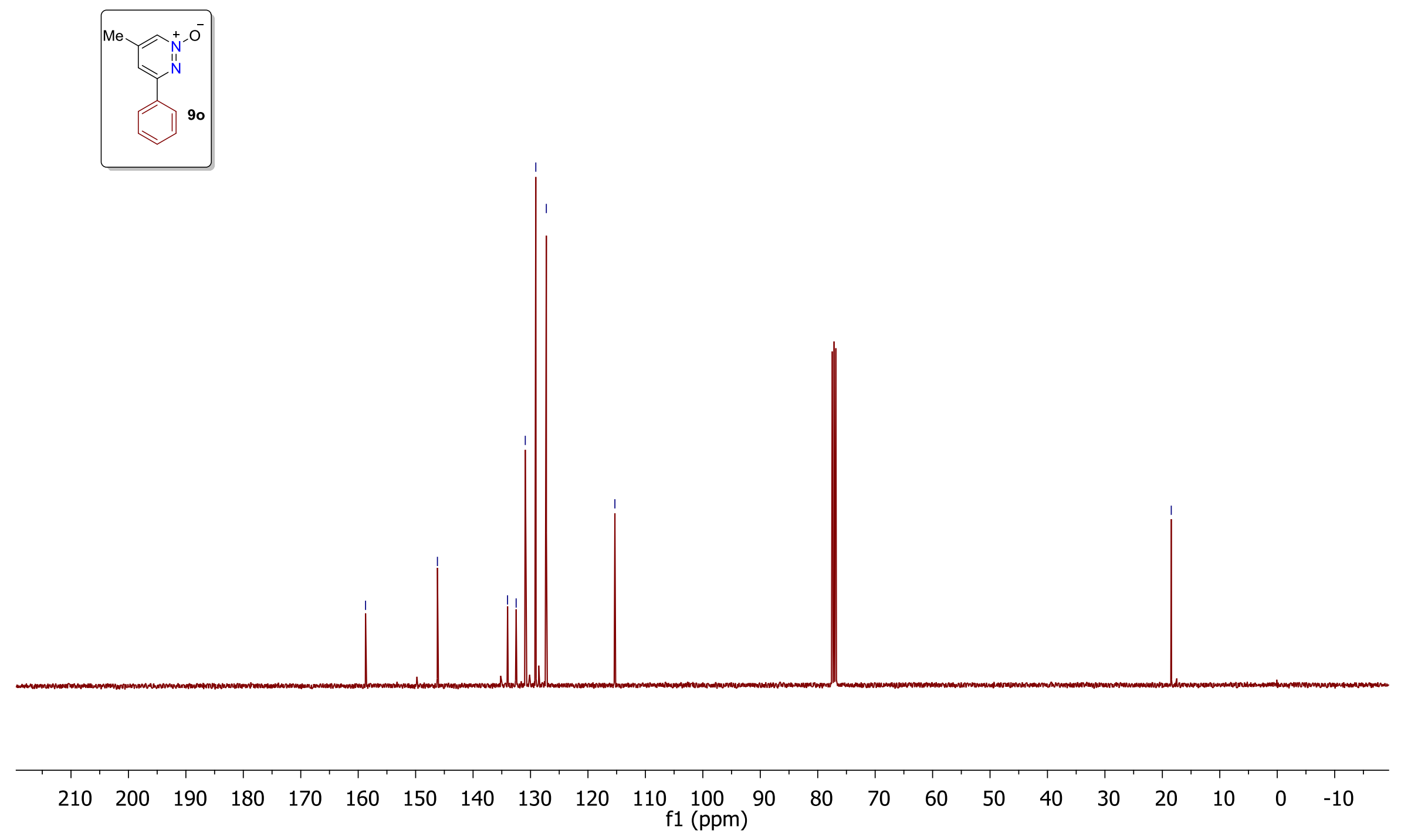
S103 


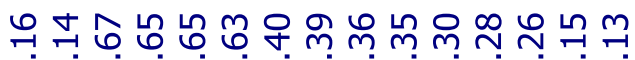

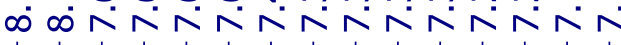
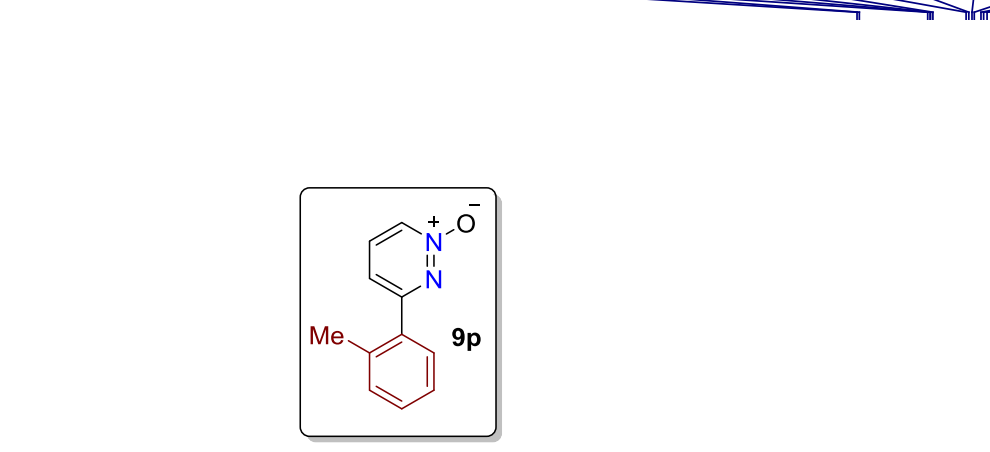

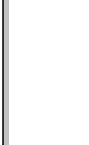
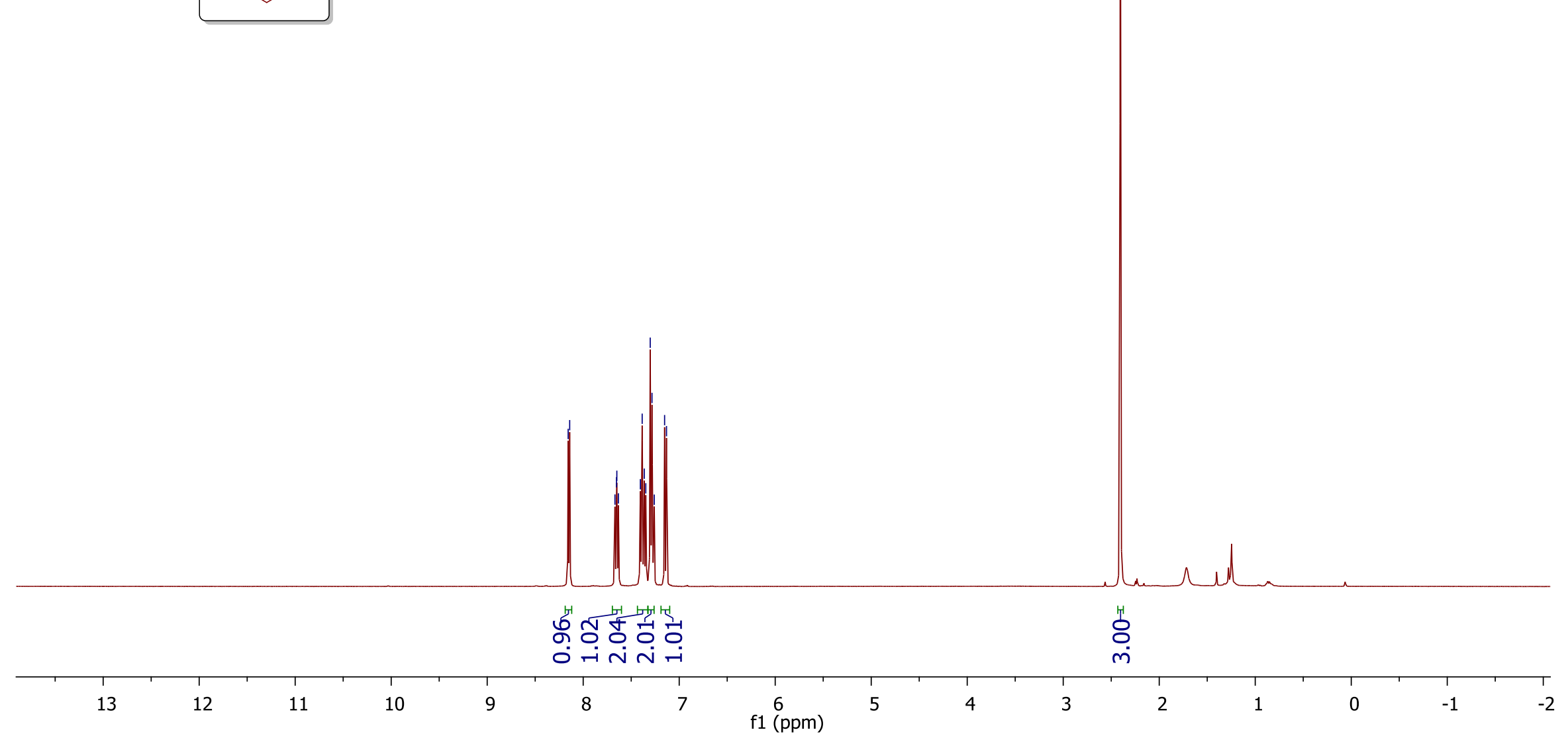


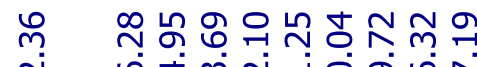

年
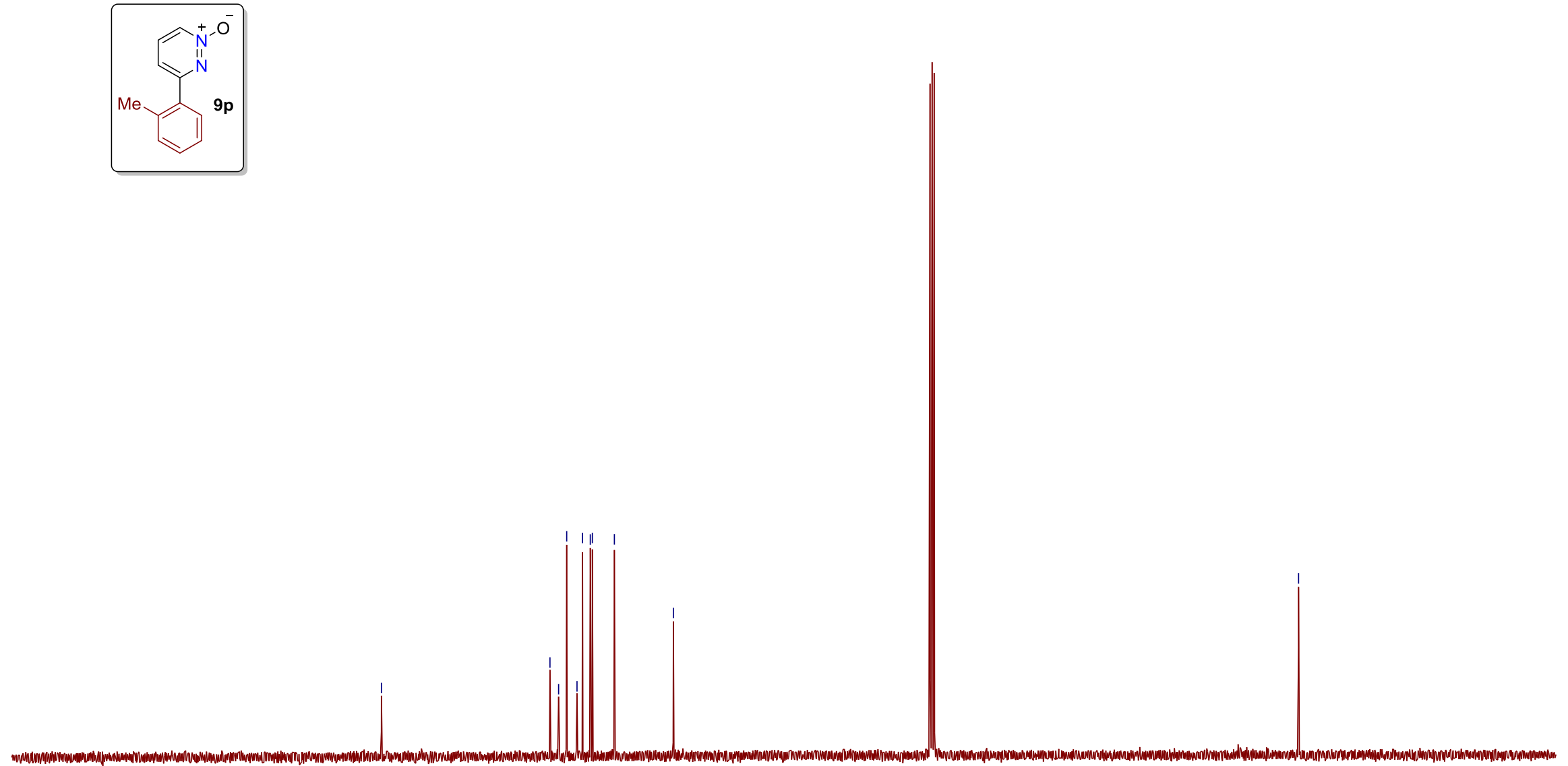


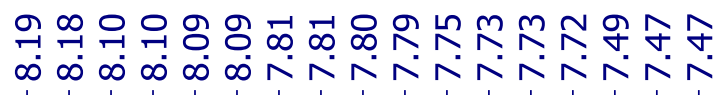
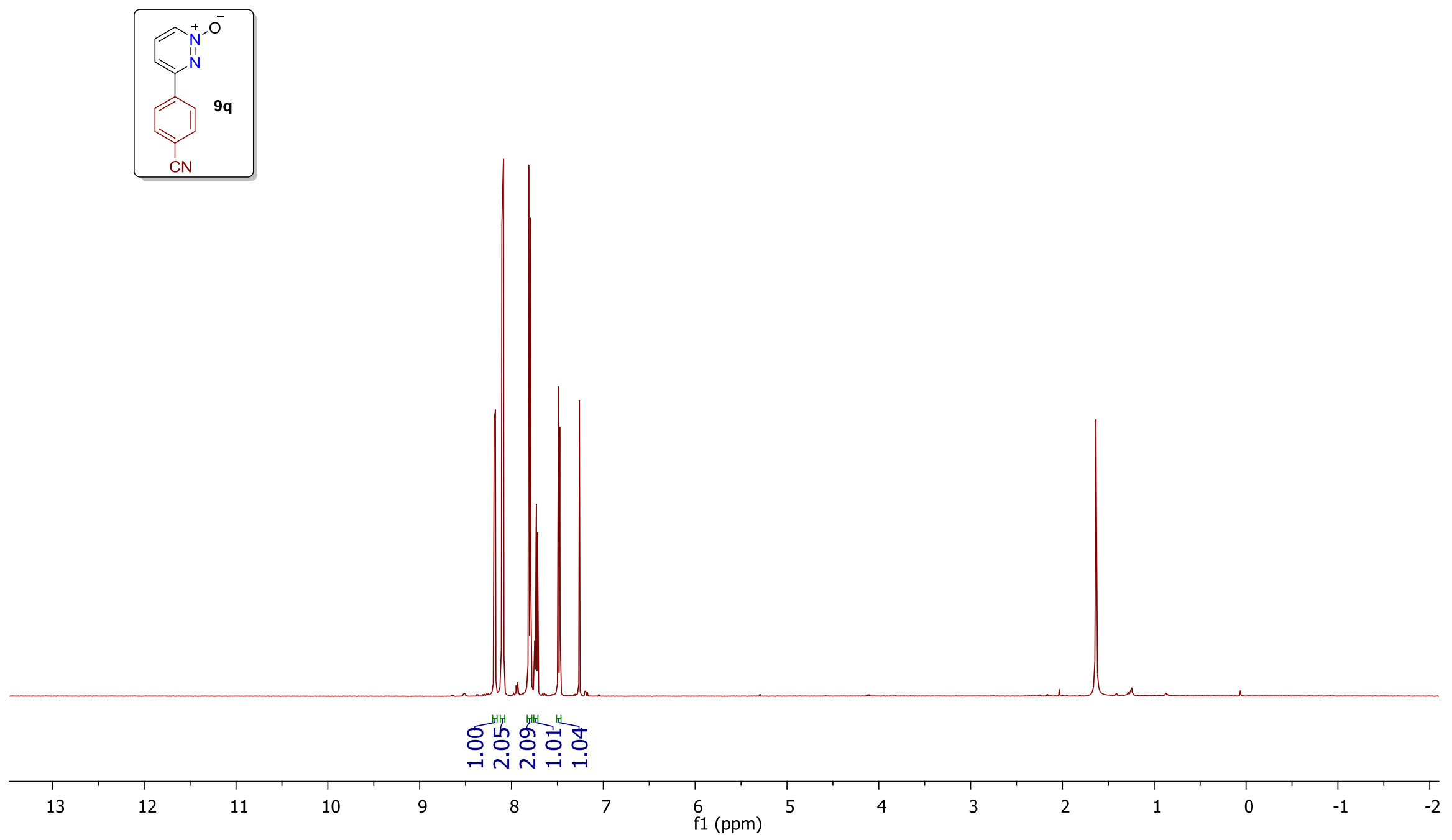

S106 


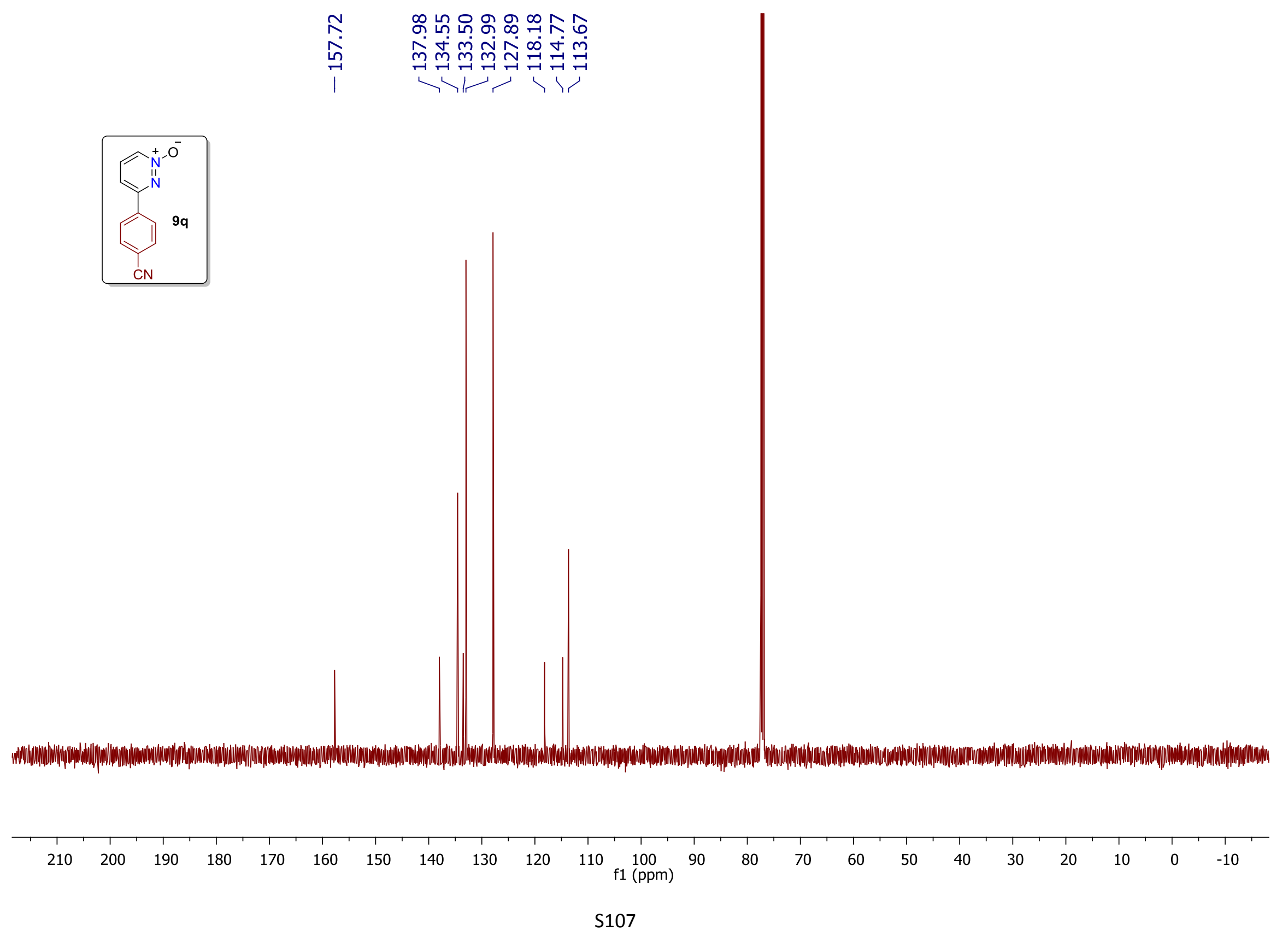


ఫ

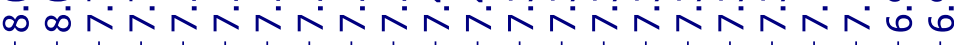
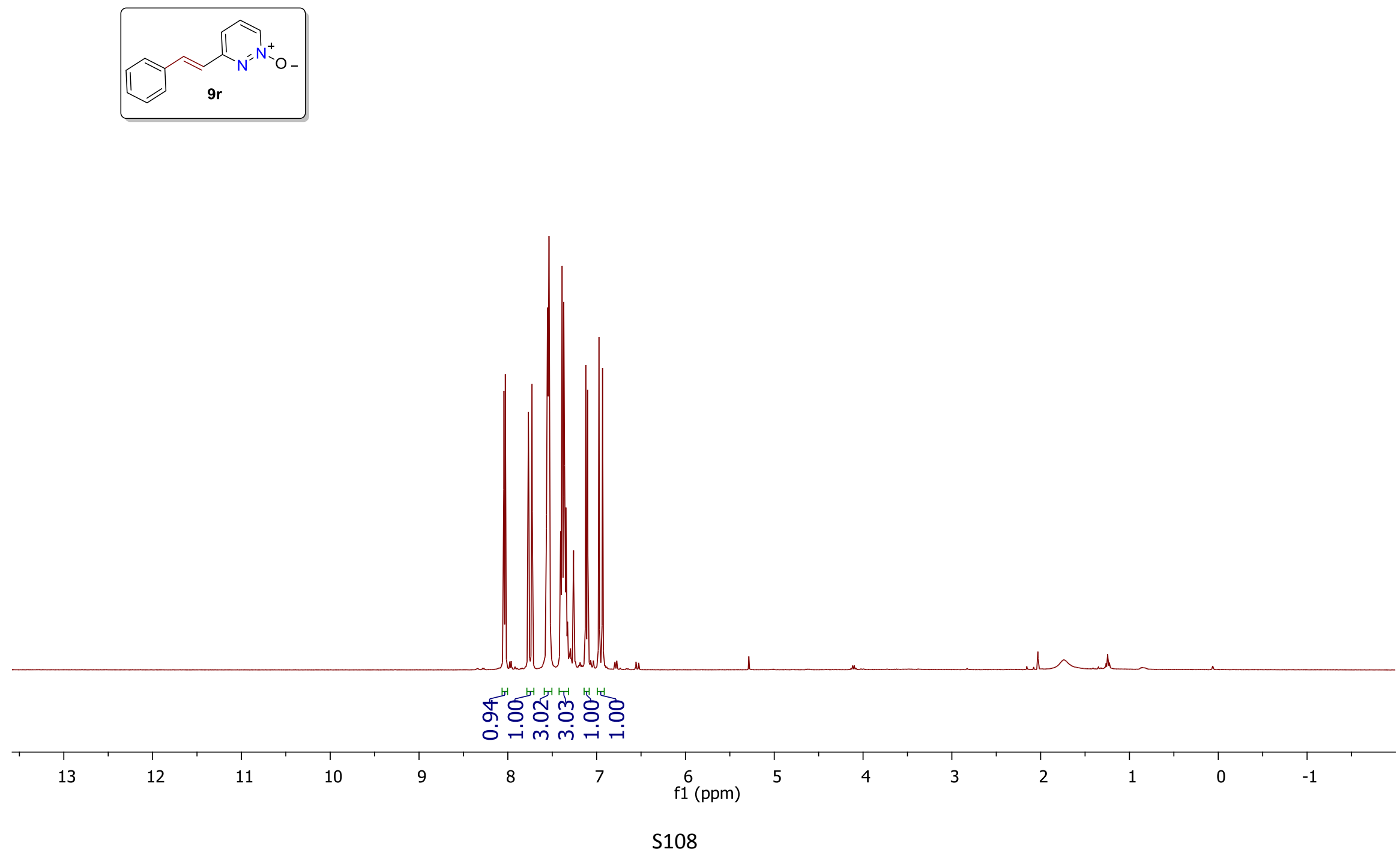


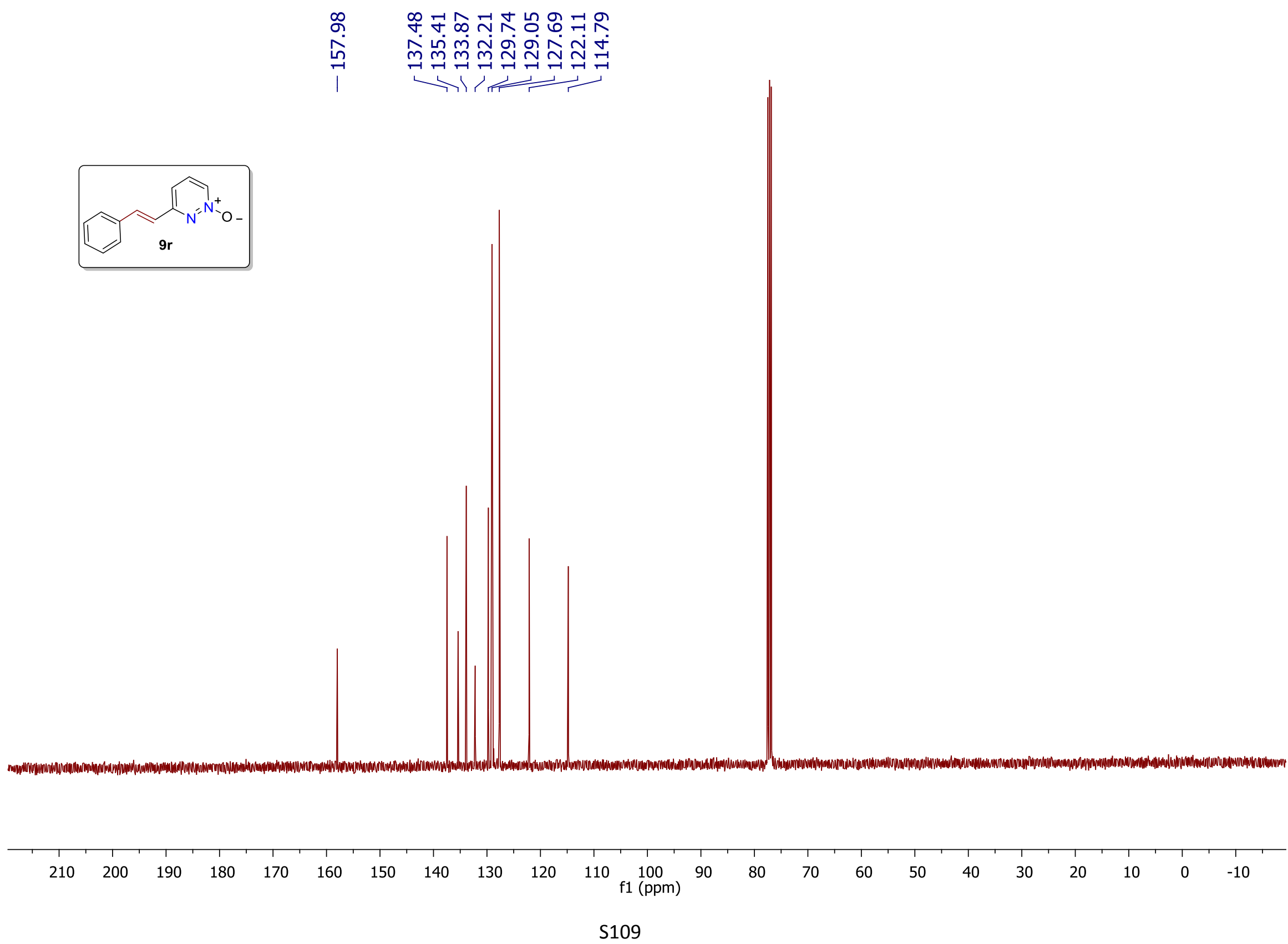




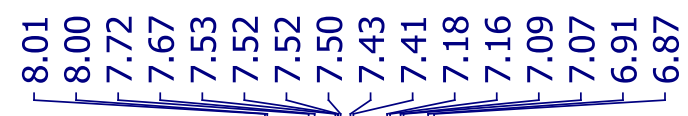
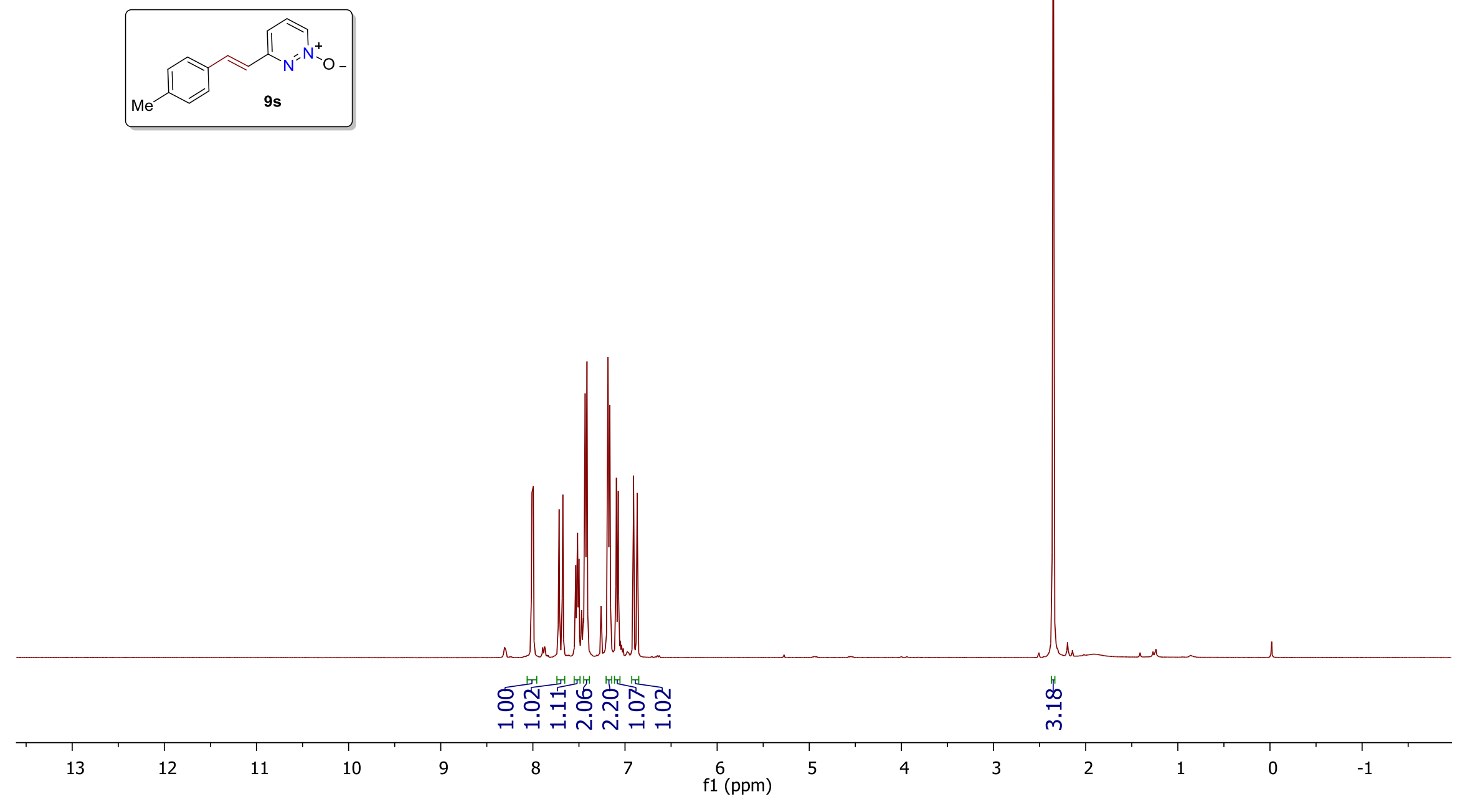

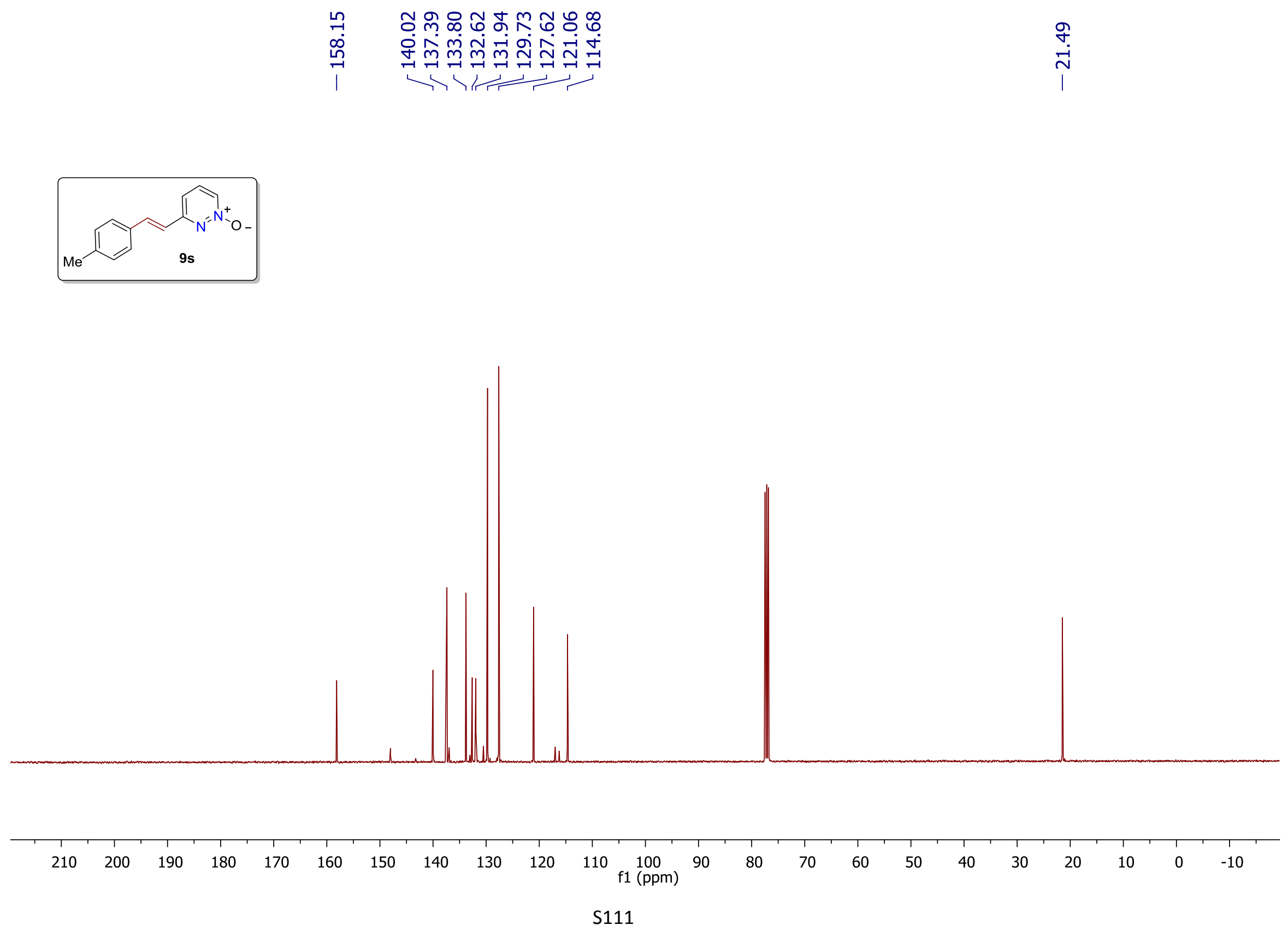


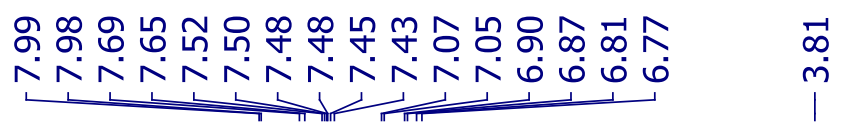

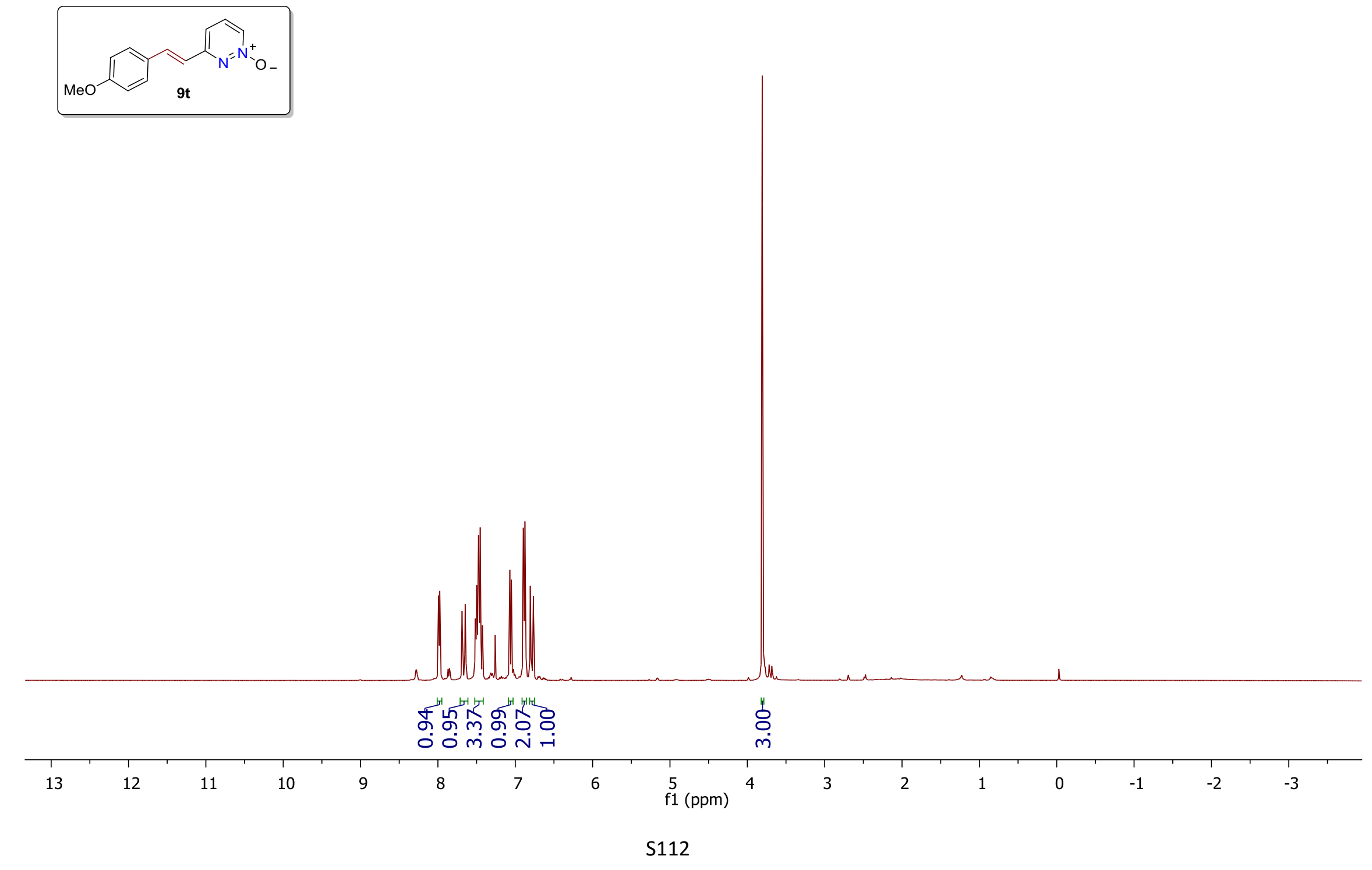



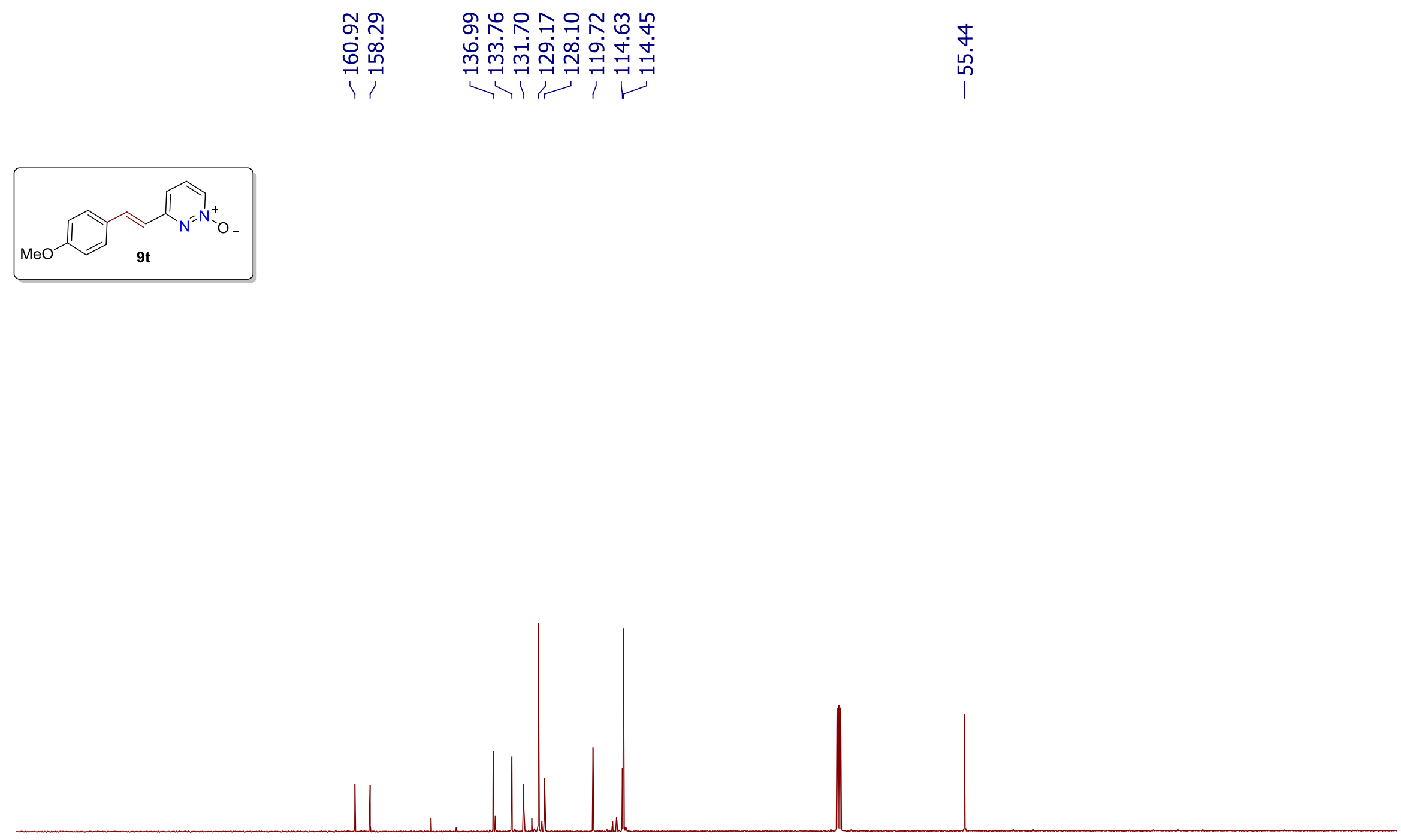


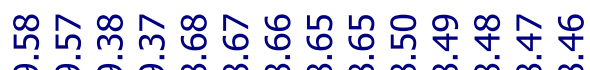
नं नं $\sigma^{\circ} \infty \infty^{\circ} \infty \infty^{\circ} \infty \infty \infty \infty$

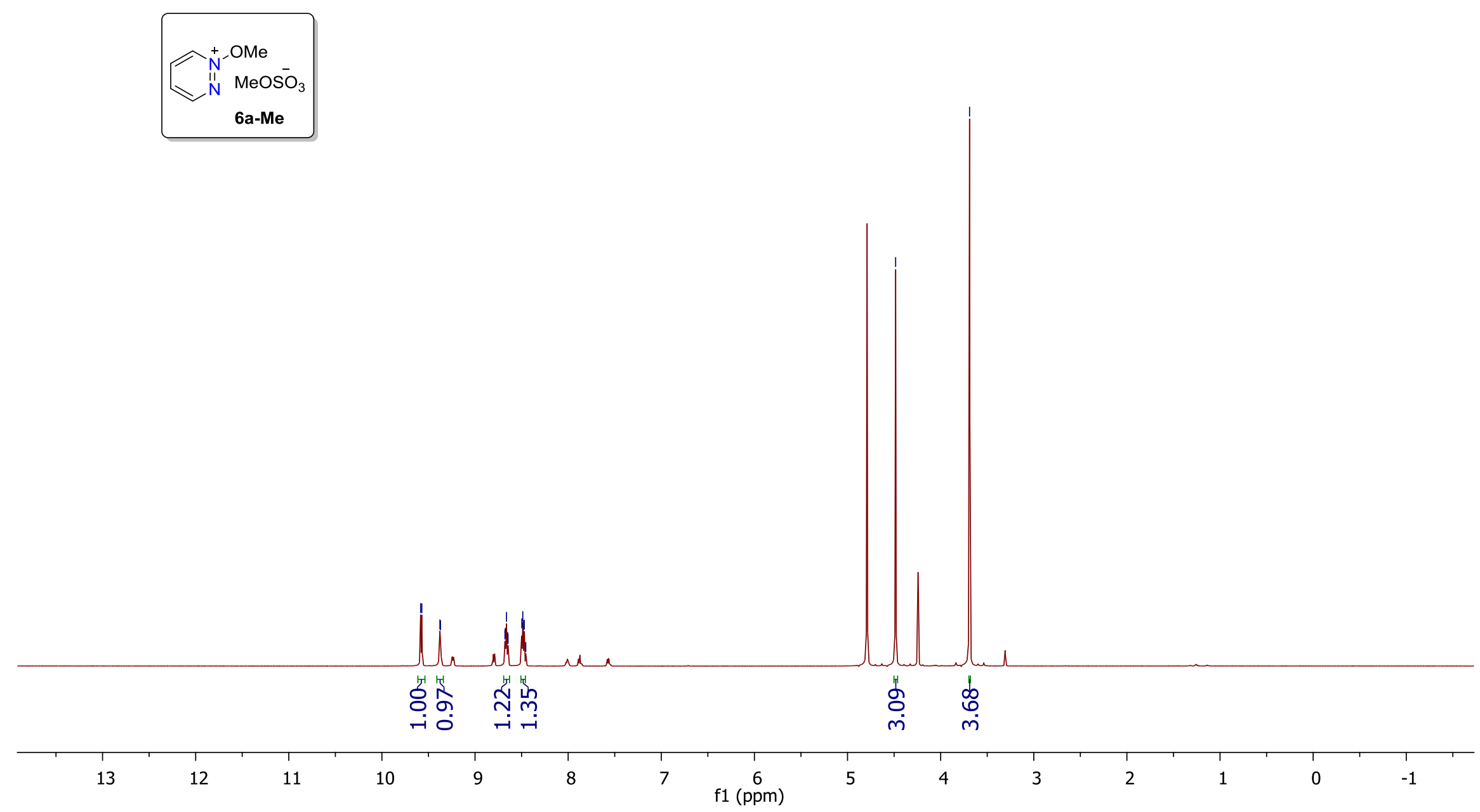




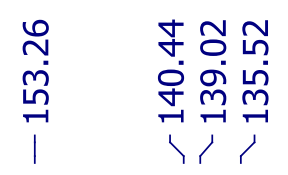

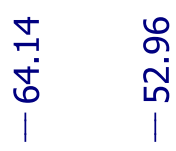

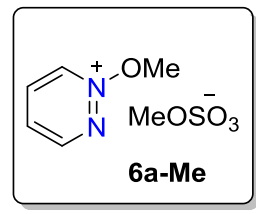

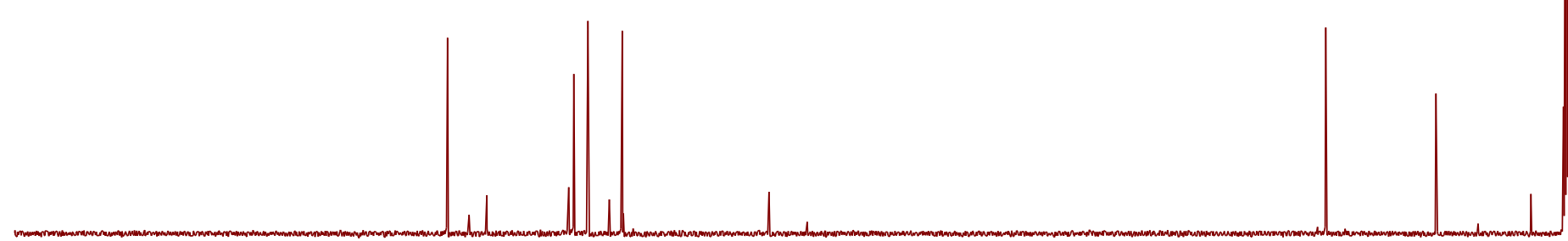

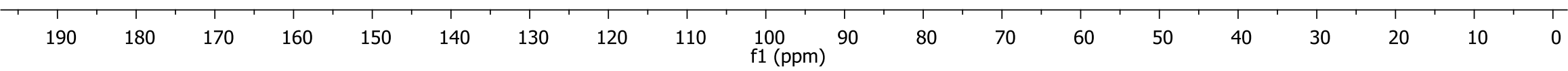




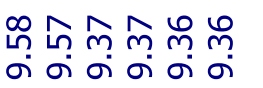
a g a

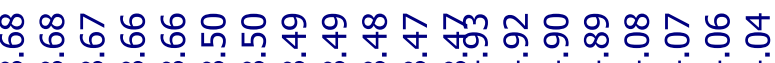

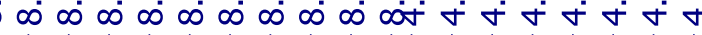

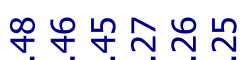

iriririr

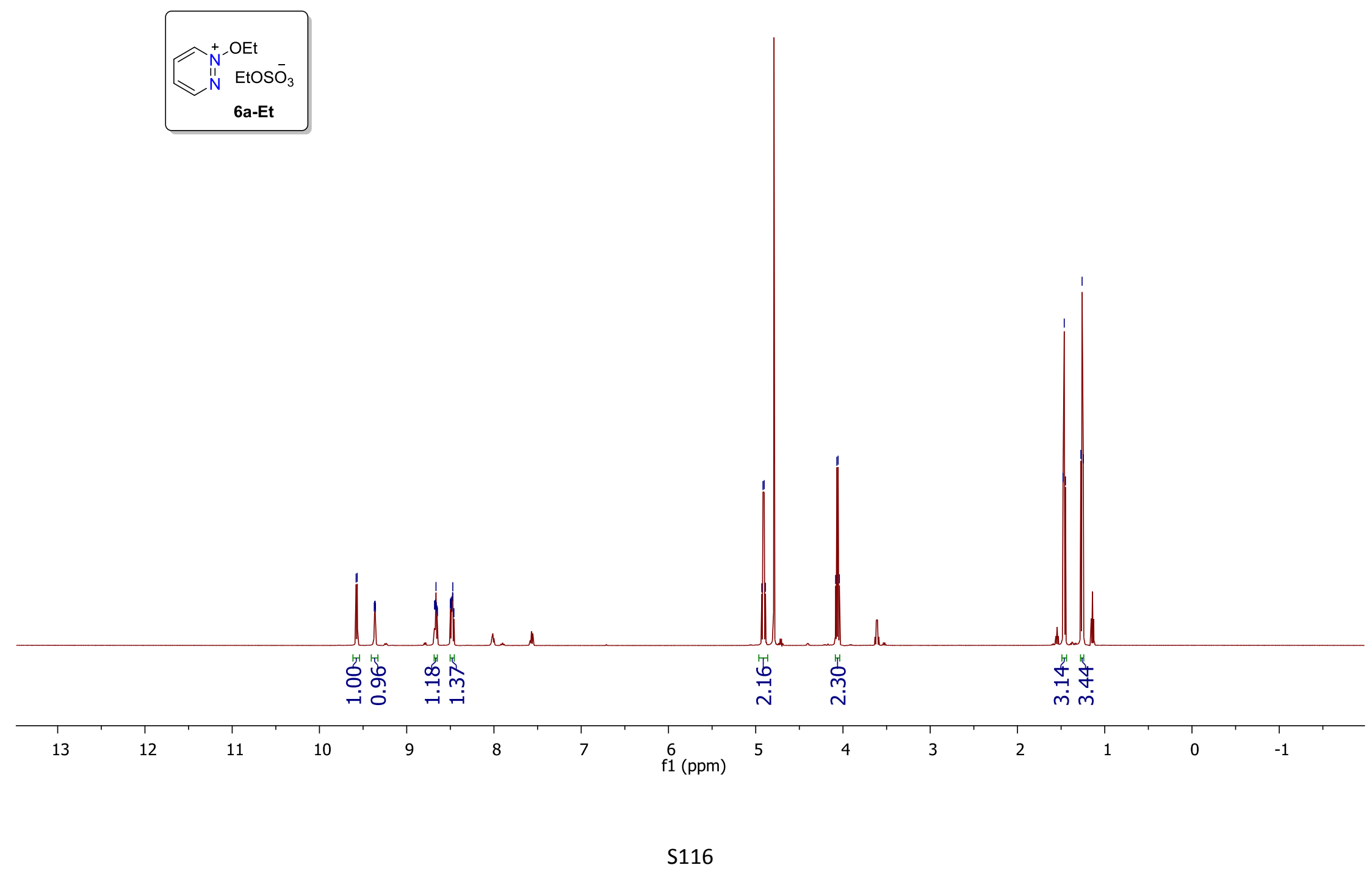




$$
\begin{aligned}
& \text { ㅇ 유음 } \\
& \text { กี } \\
& \text { I } 11
\end{aligned}
$$

ํำ

ํำ ㄱำ

$$
\begin{aligned}
& \text { Cot } \\
& \text { 6a-Et }
\end{aligned}
$$
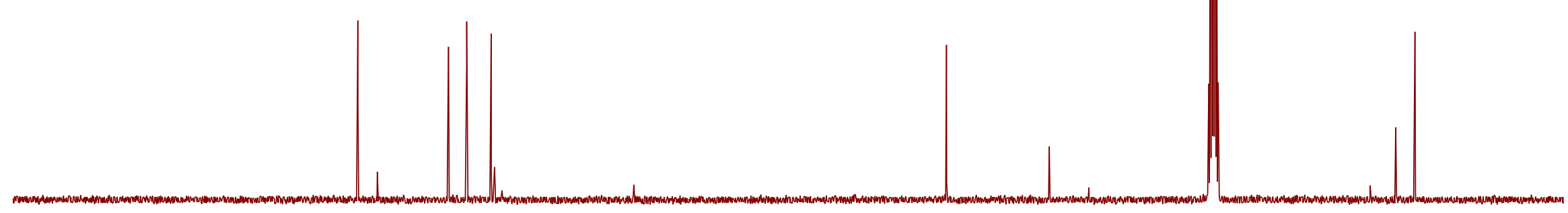


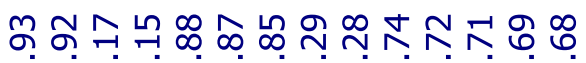

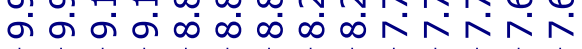

$\stackrel{\infty}{\sim}$ in
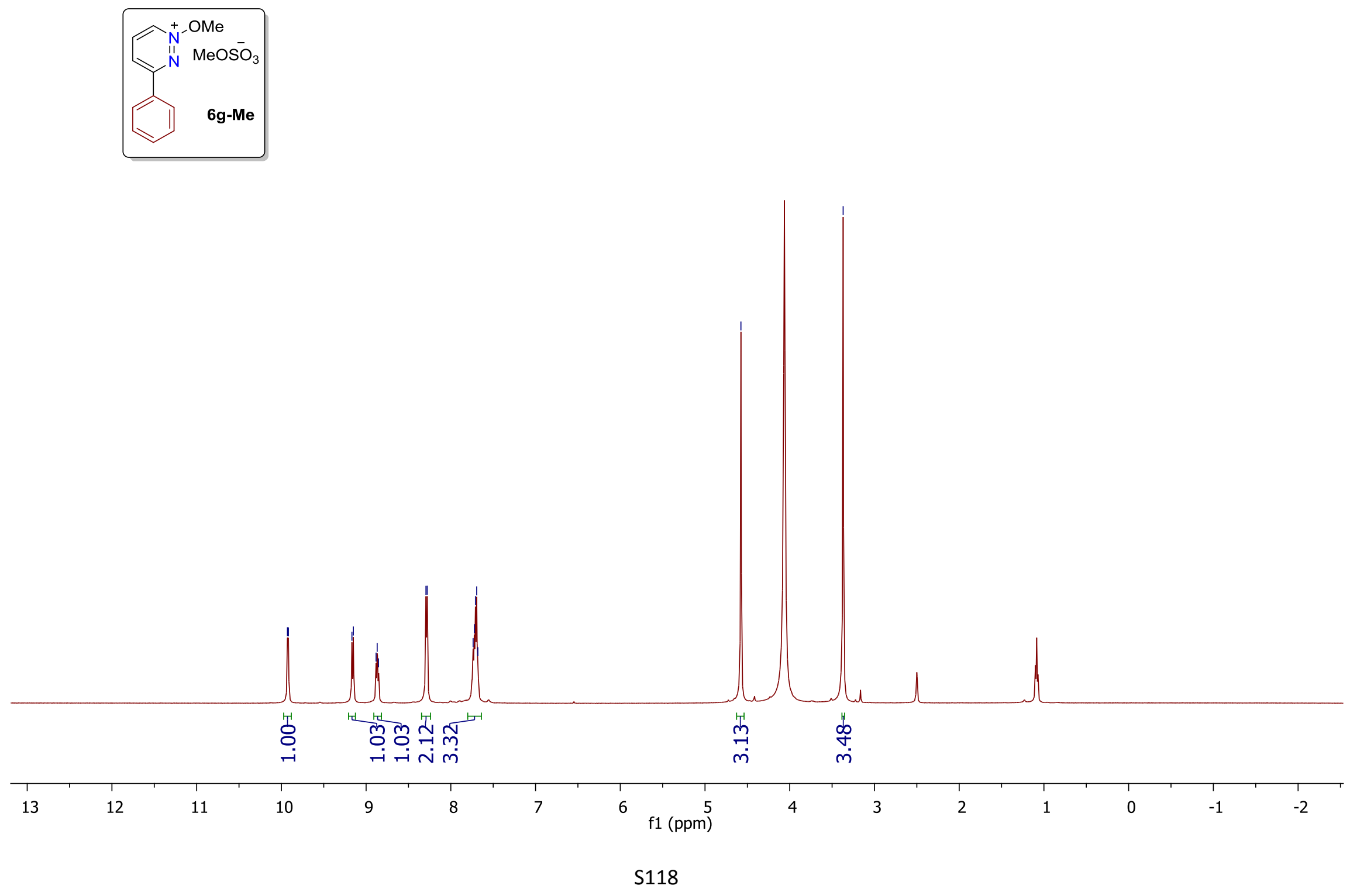


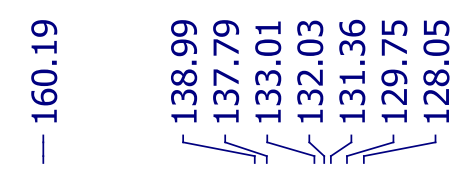

$\begin{array}{cc}\text { m } & \infty \\ \text { กิ } & \text { กี่ } \\ & 1\end{array}$

6g-Me

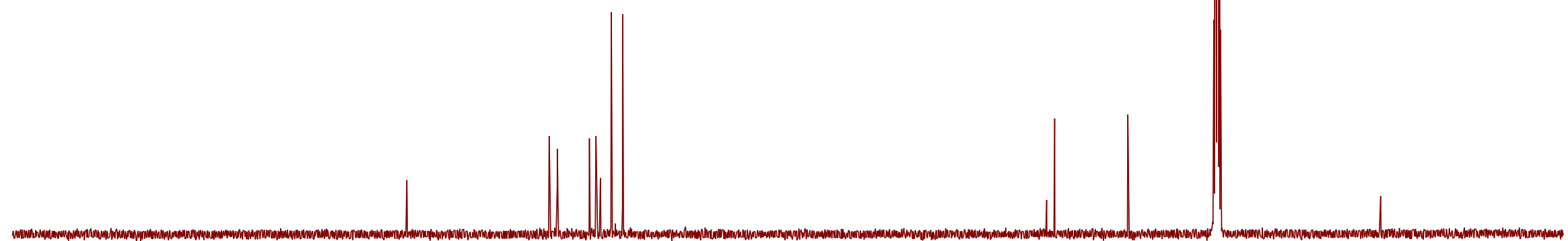

$210 \quad 200$

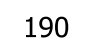

180

170

$160 \quad 150$

140

130

120

$110 \quad 100$

$90 \quad 80$

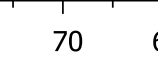

60

50

$40-30$

$\begin{array}{lllll} & & 1 & 1 \\ & 20 & 10 & 0 & -10\end{array}$ 
mิn

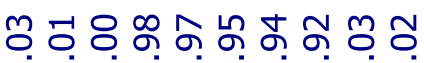

ตं

กर

ب

$$
\overbrace{\mathrm{N}_{2}} \mathrm{~N}_{\mathrm{7a}}
$$

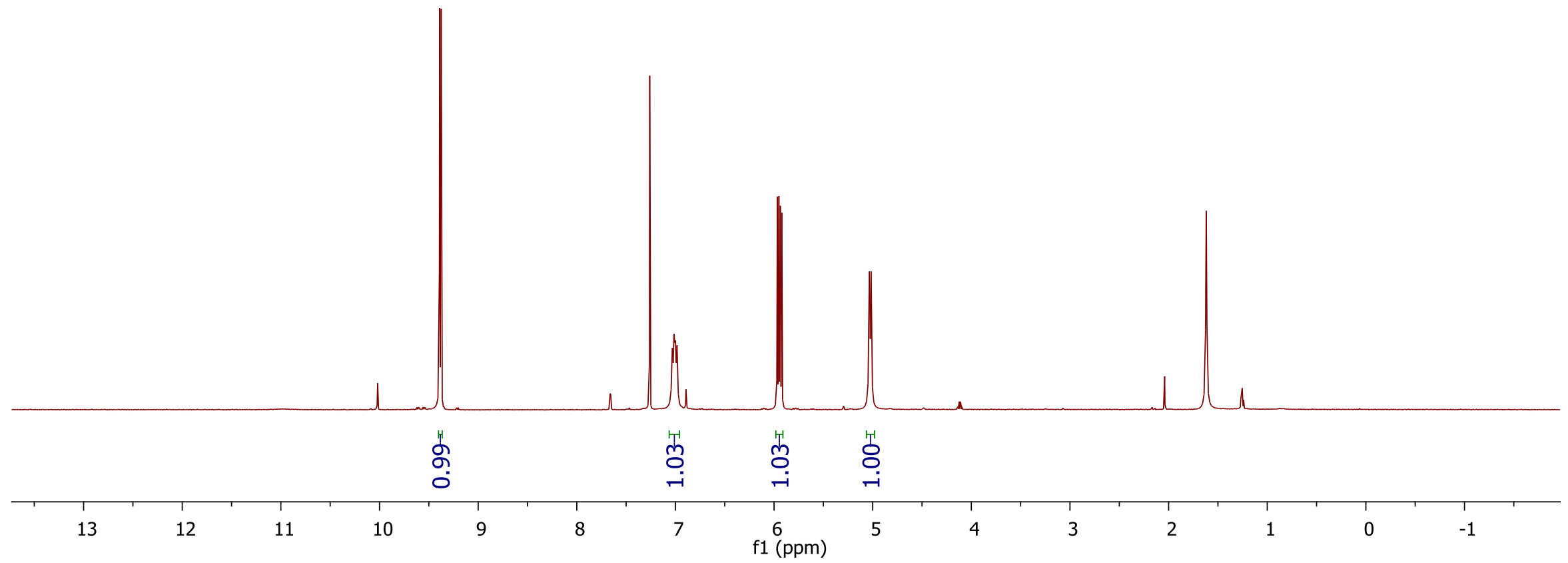



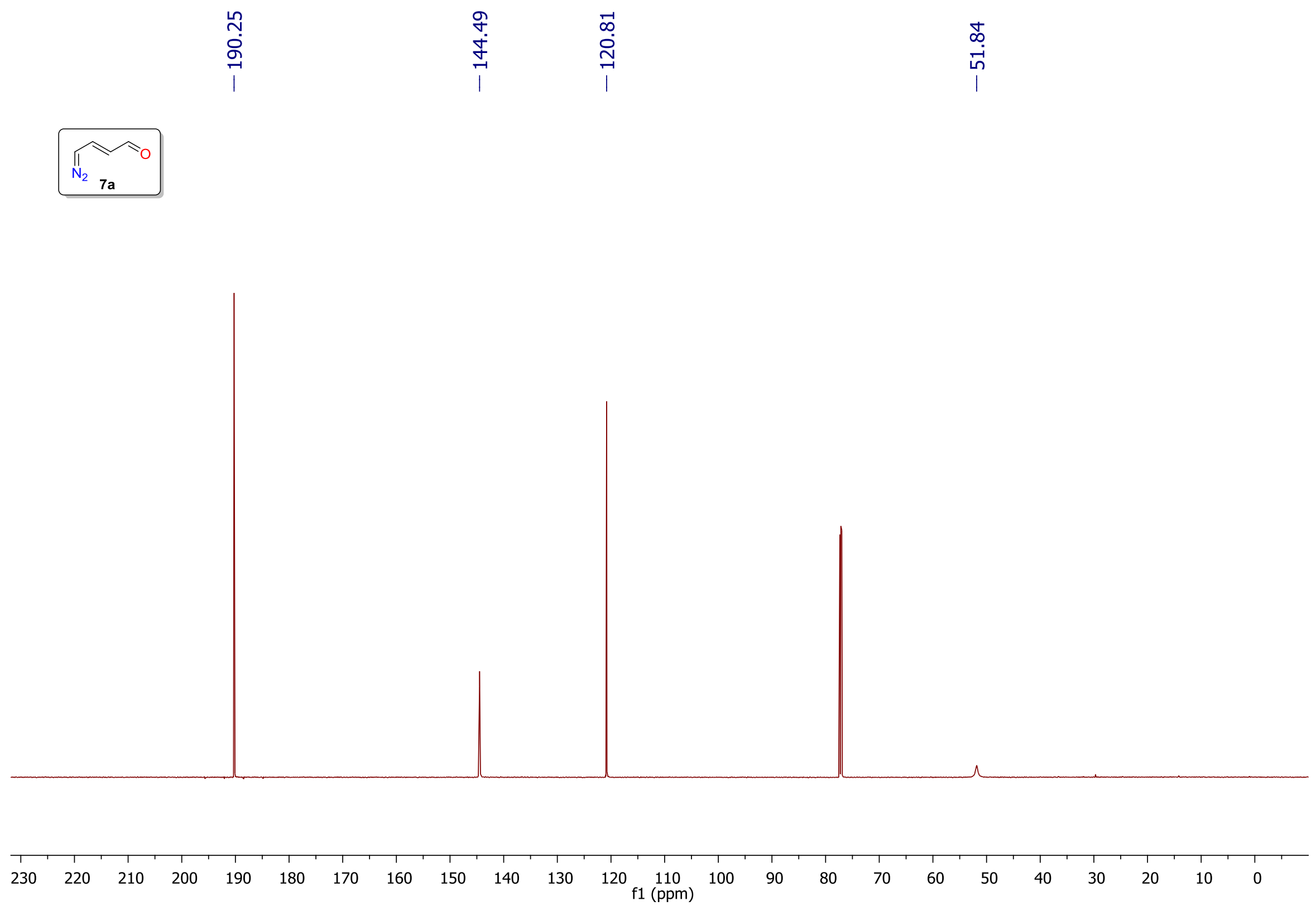


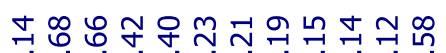

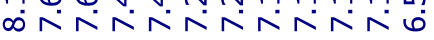
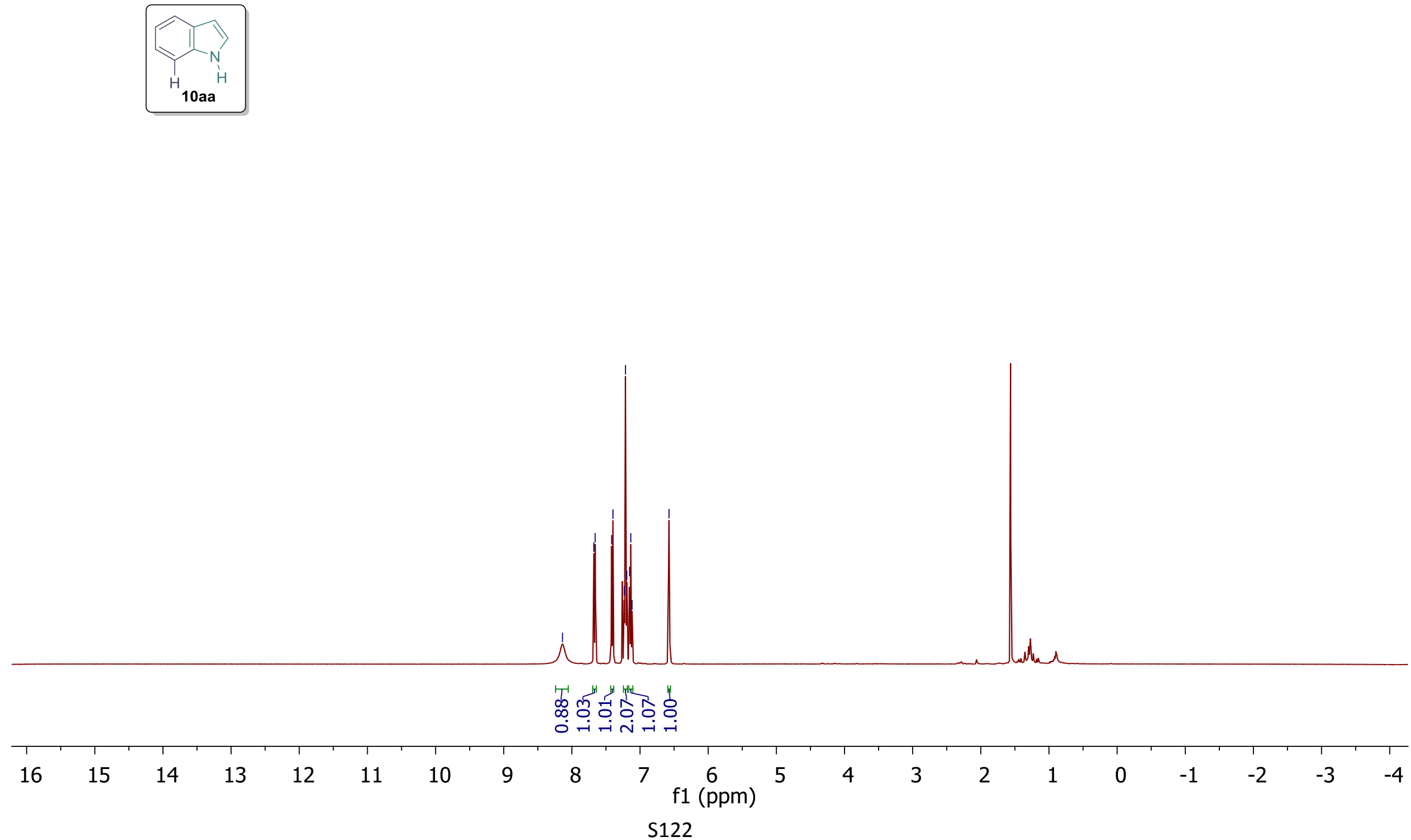


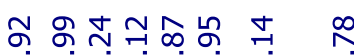

|
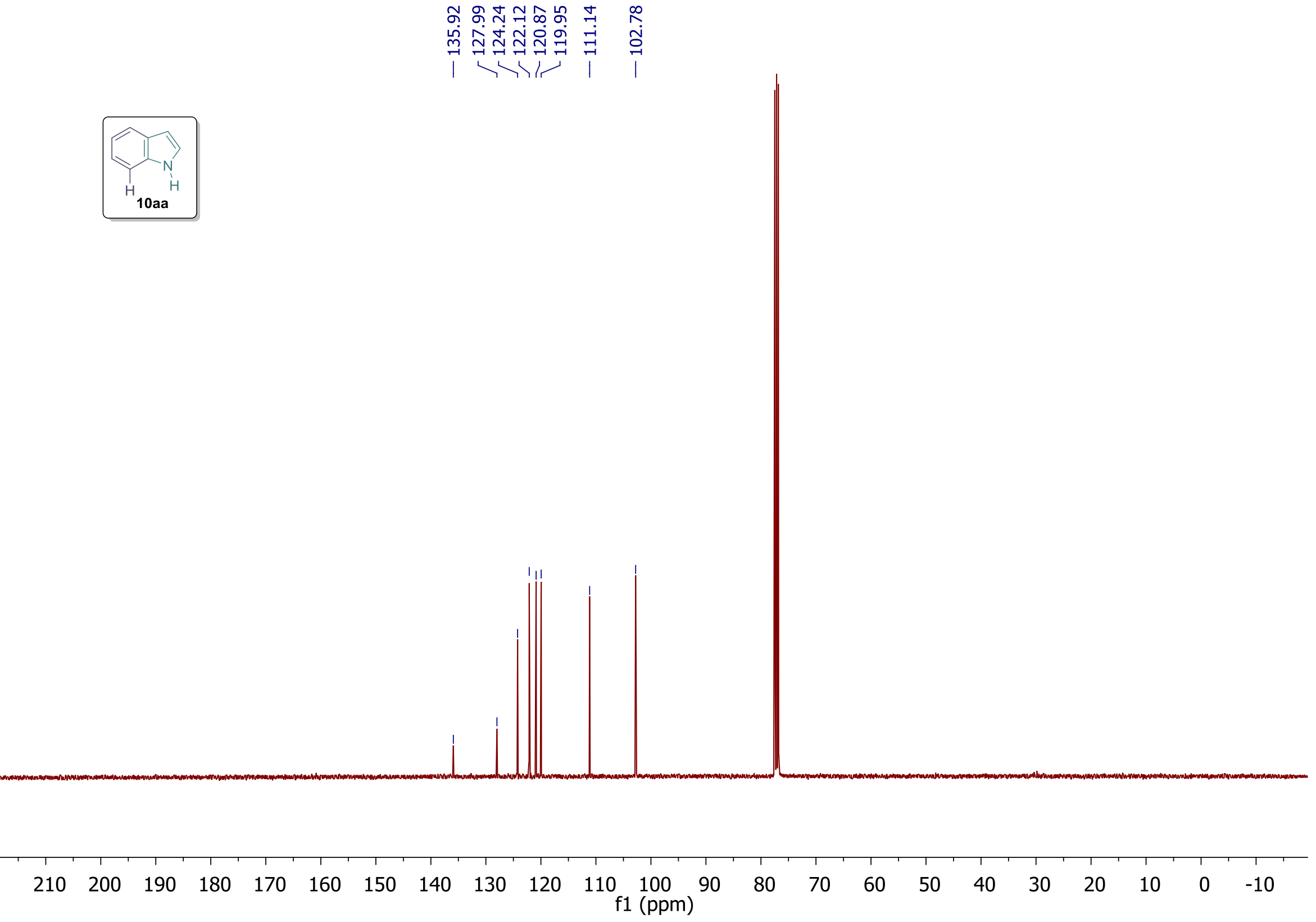


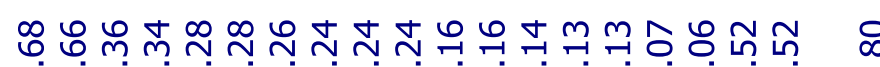

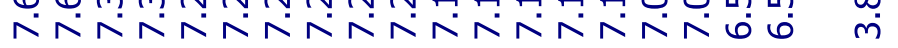
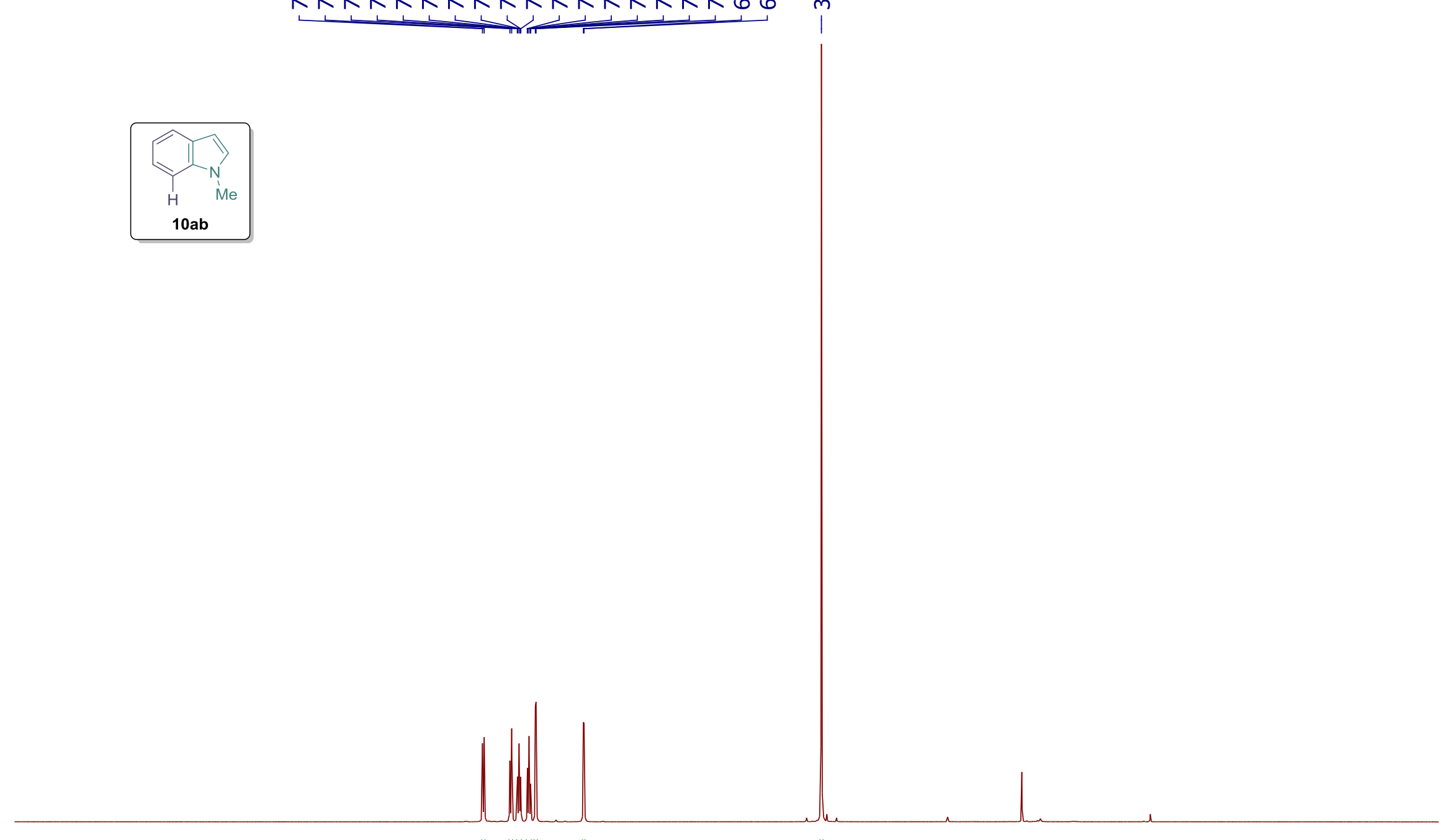

$\stackrel{\text { N }}{n}$

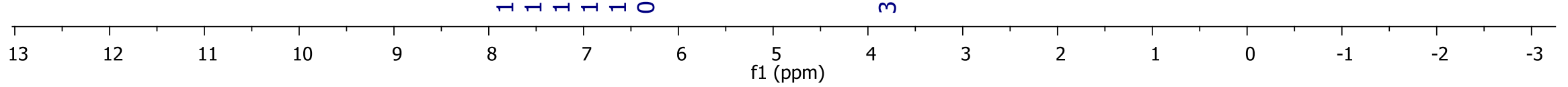




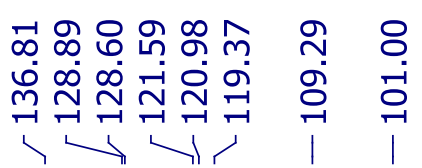

욱
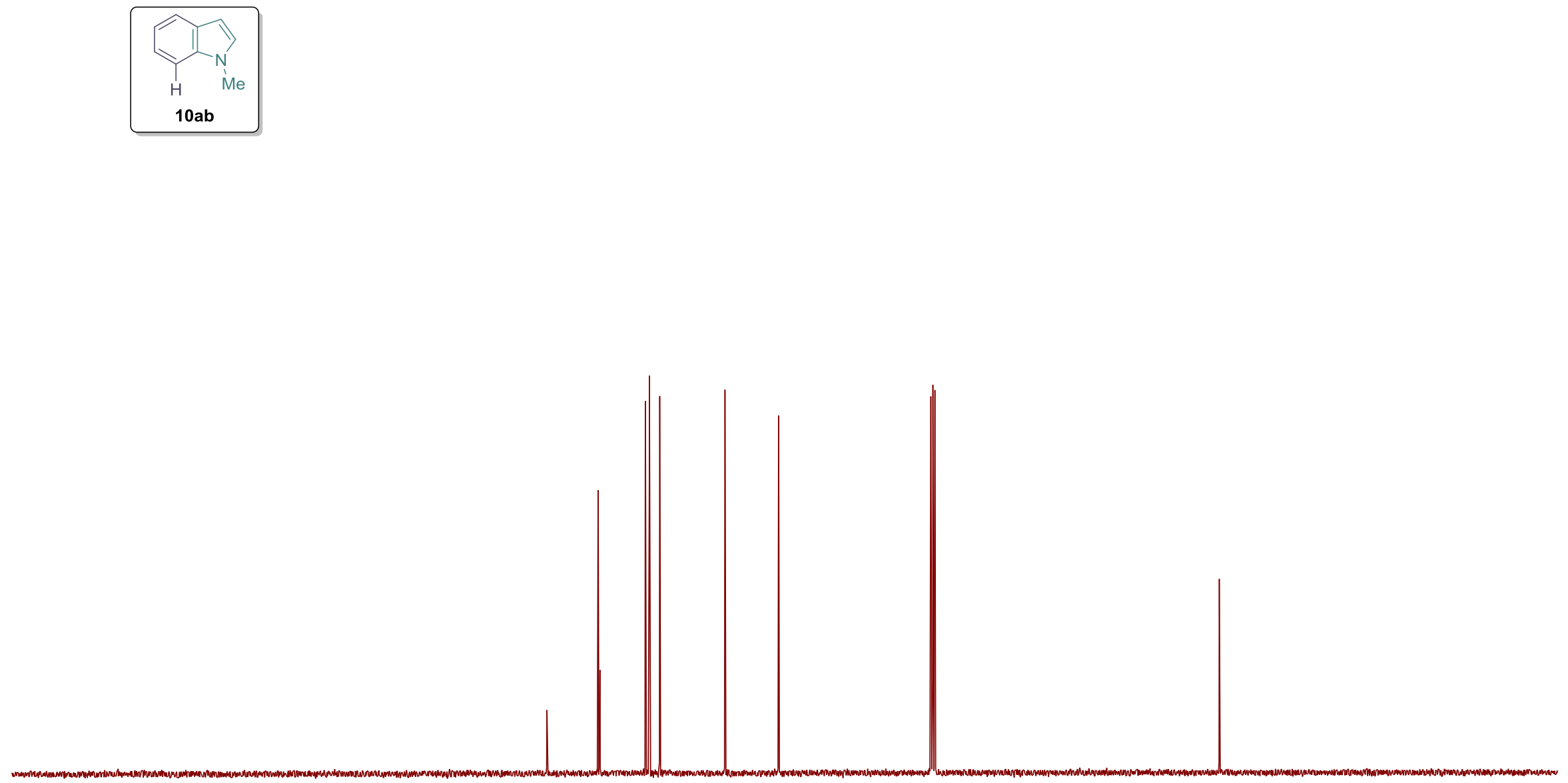


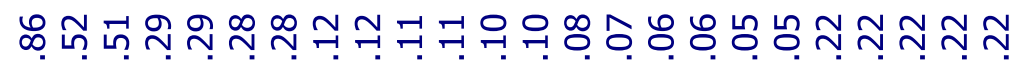

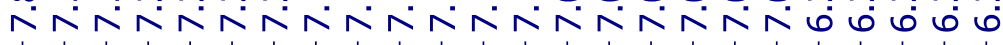
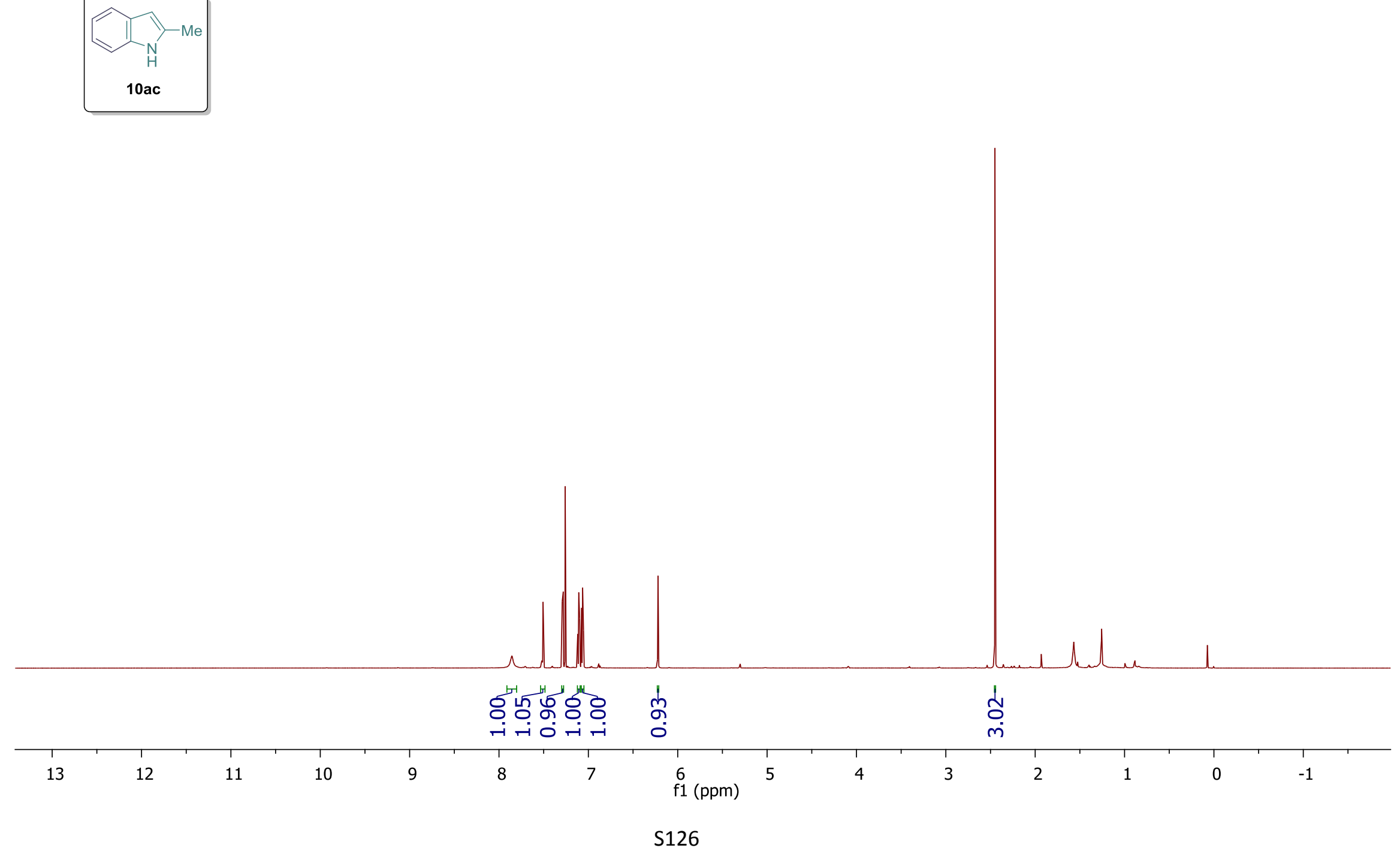


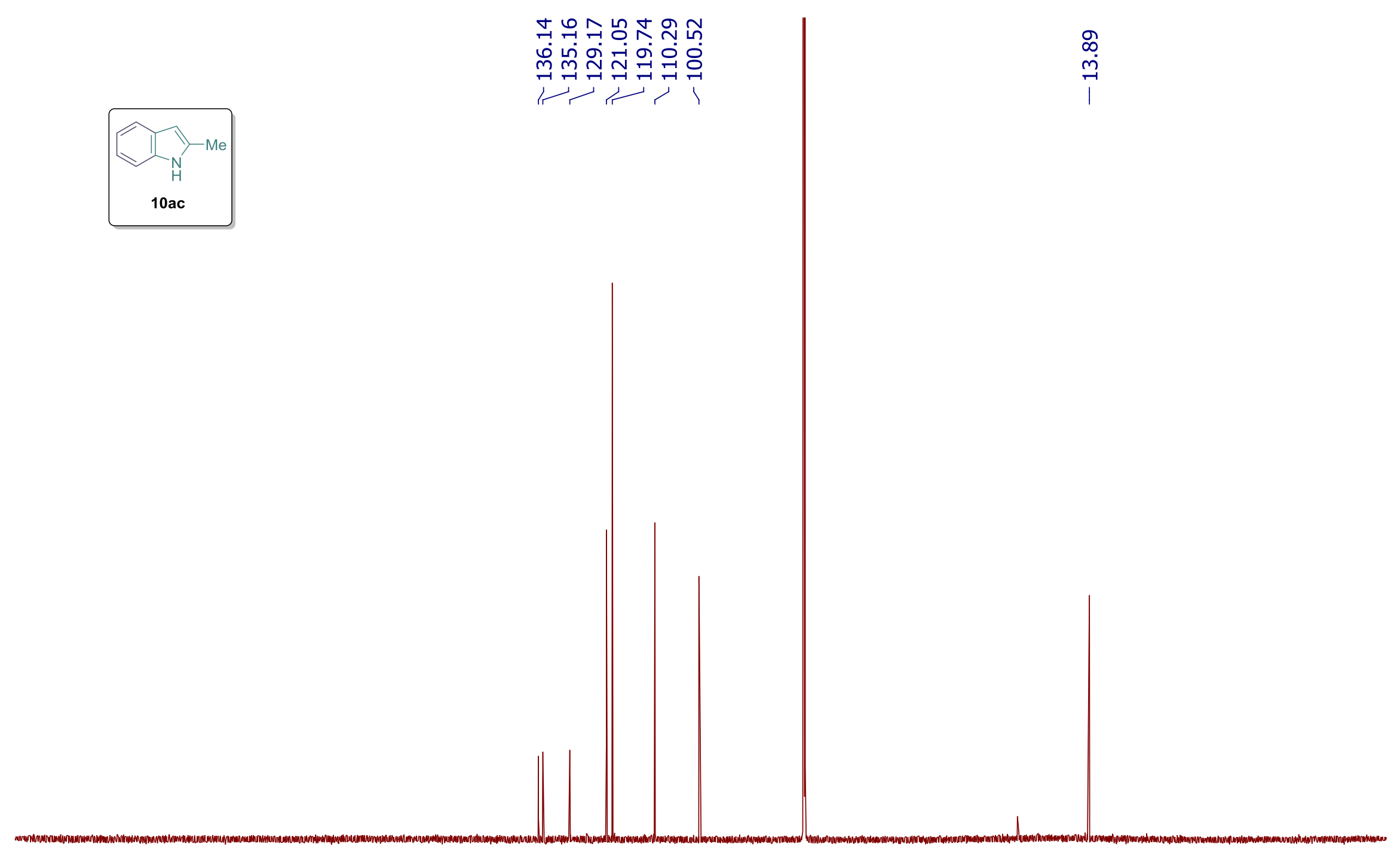

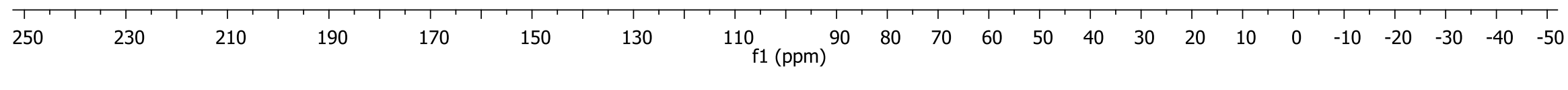




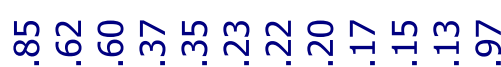

NNNNNNNÑ
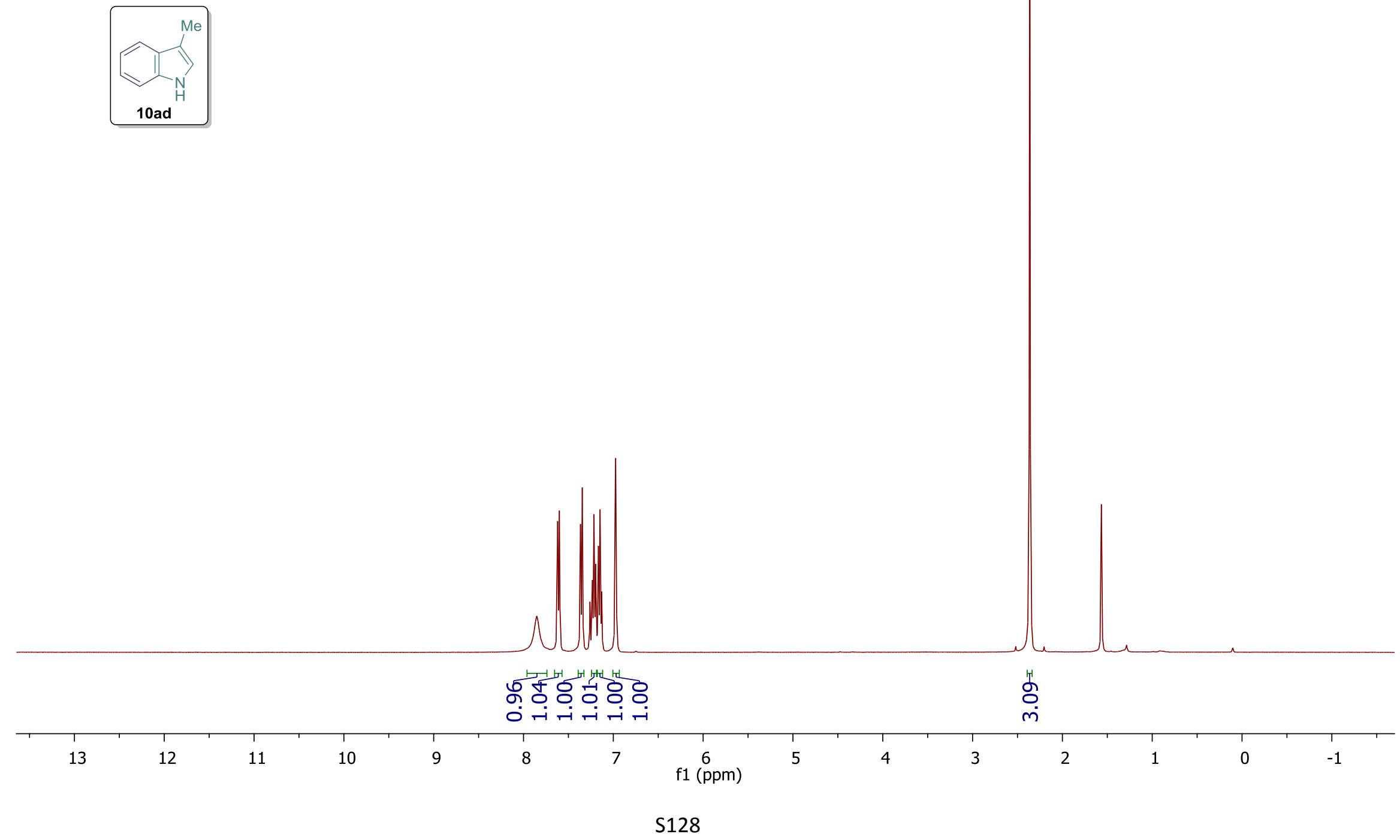


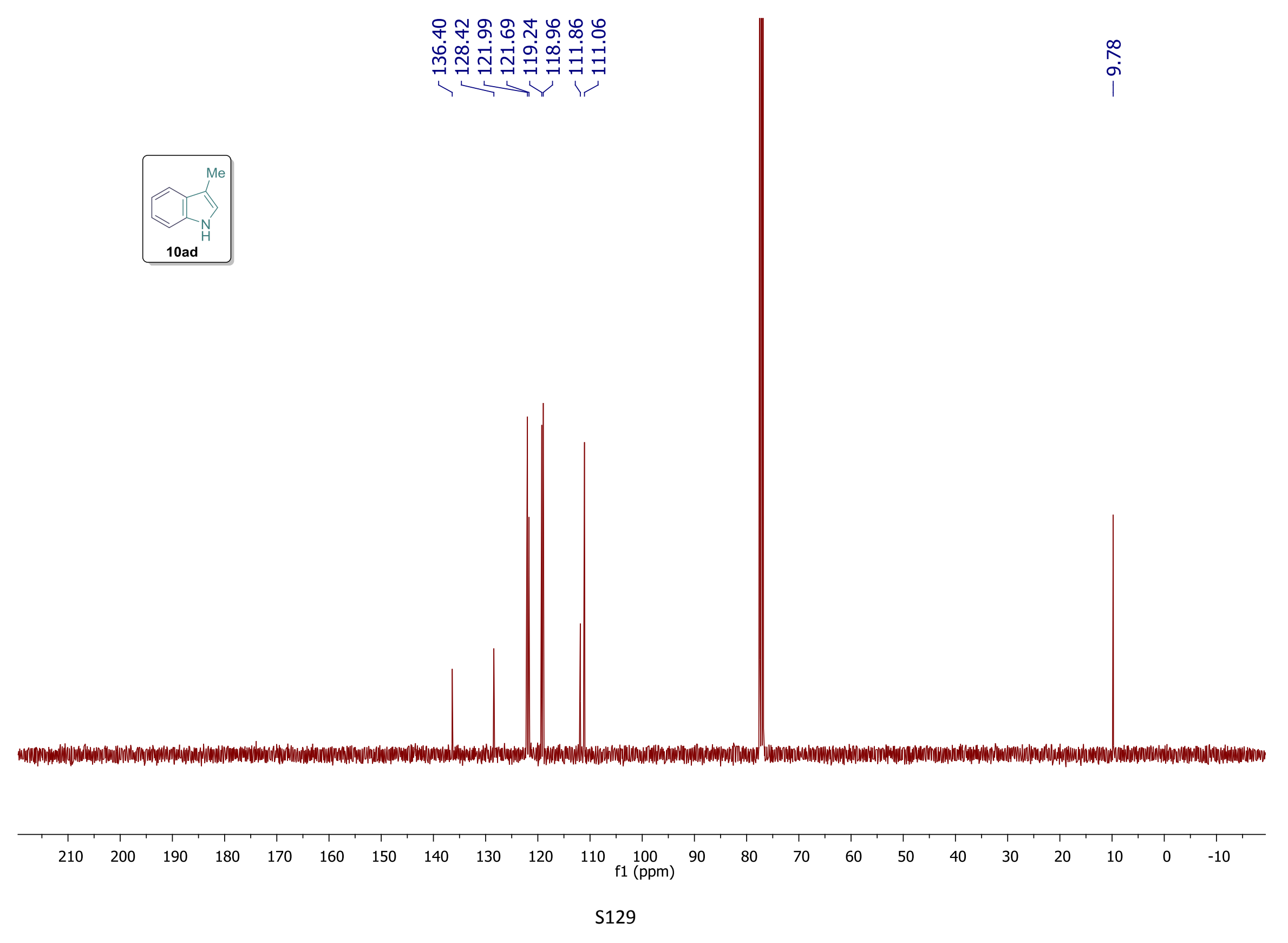



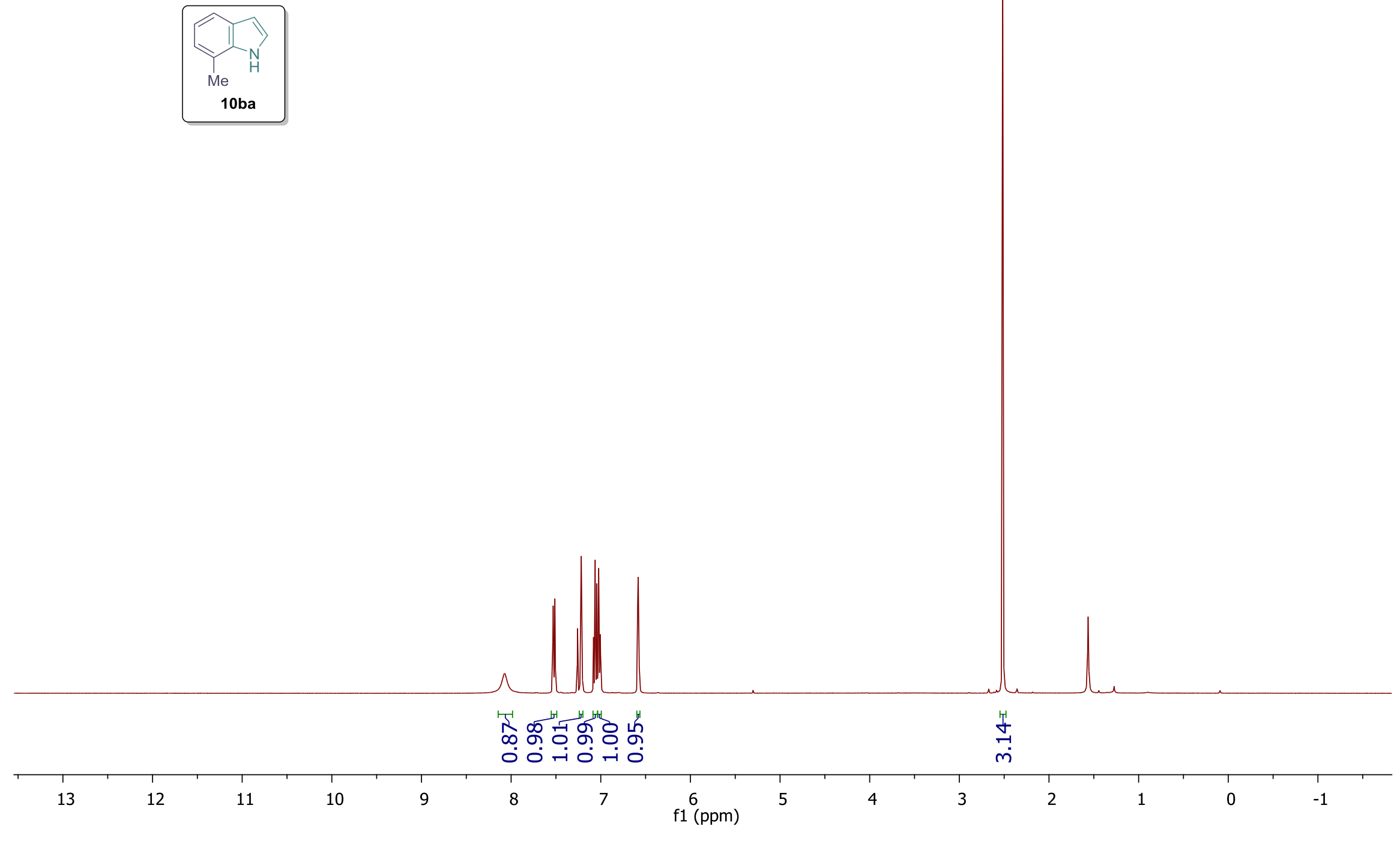


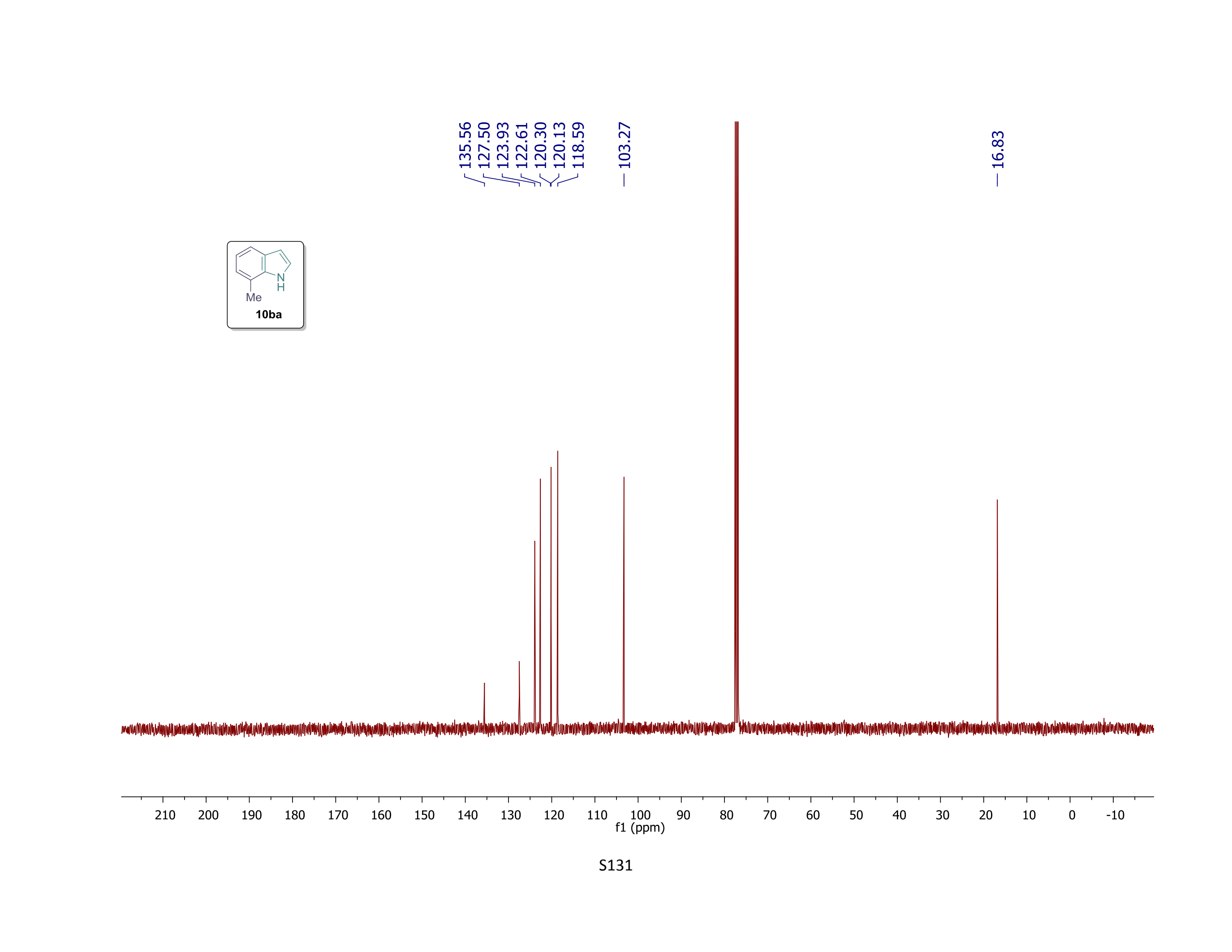




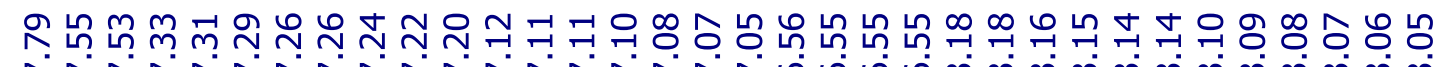

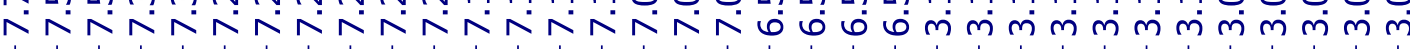
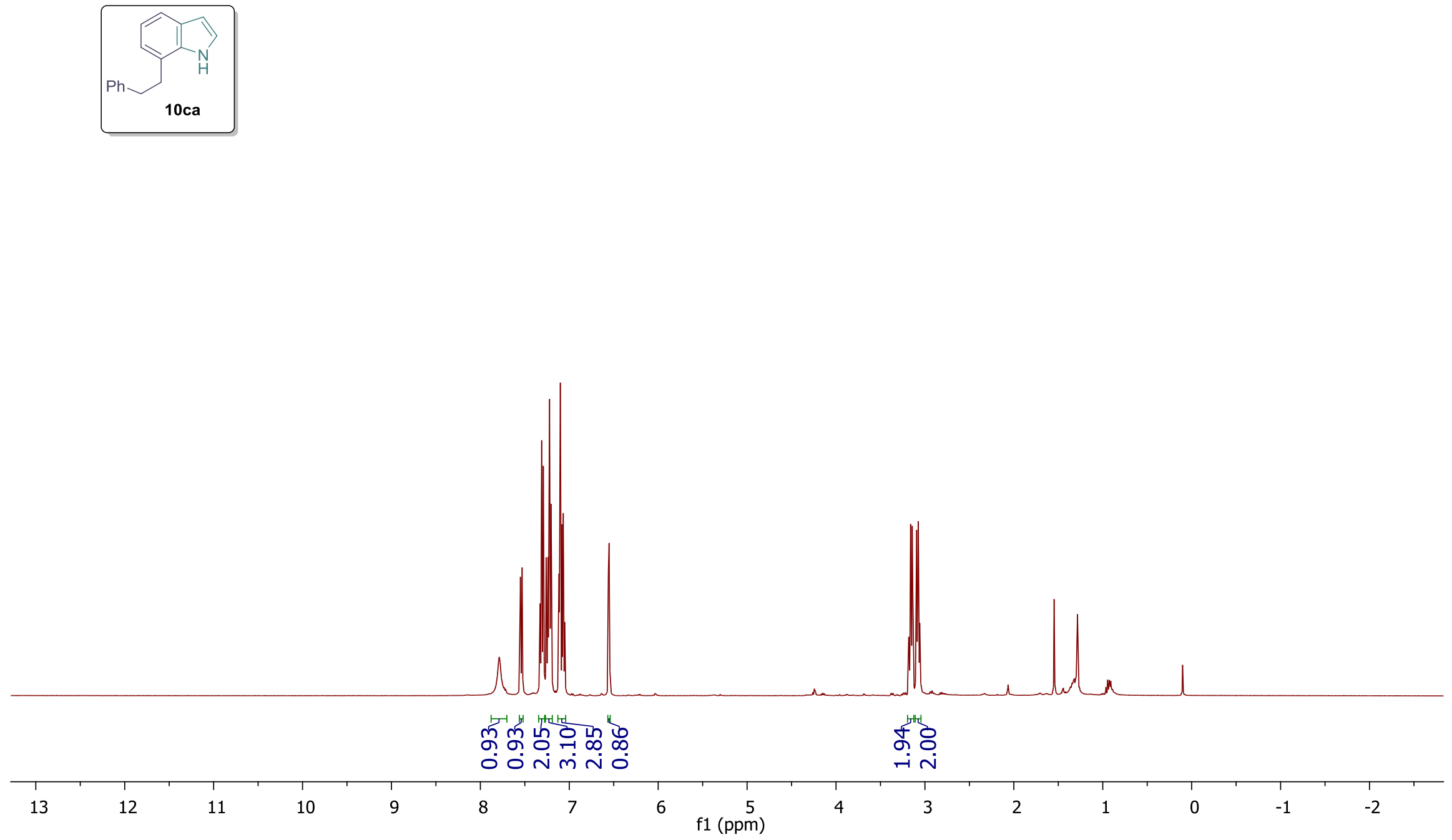


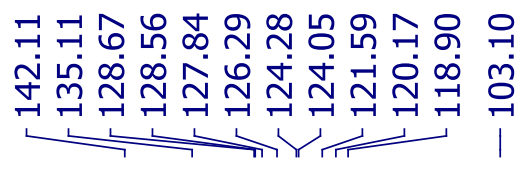

min
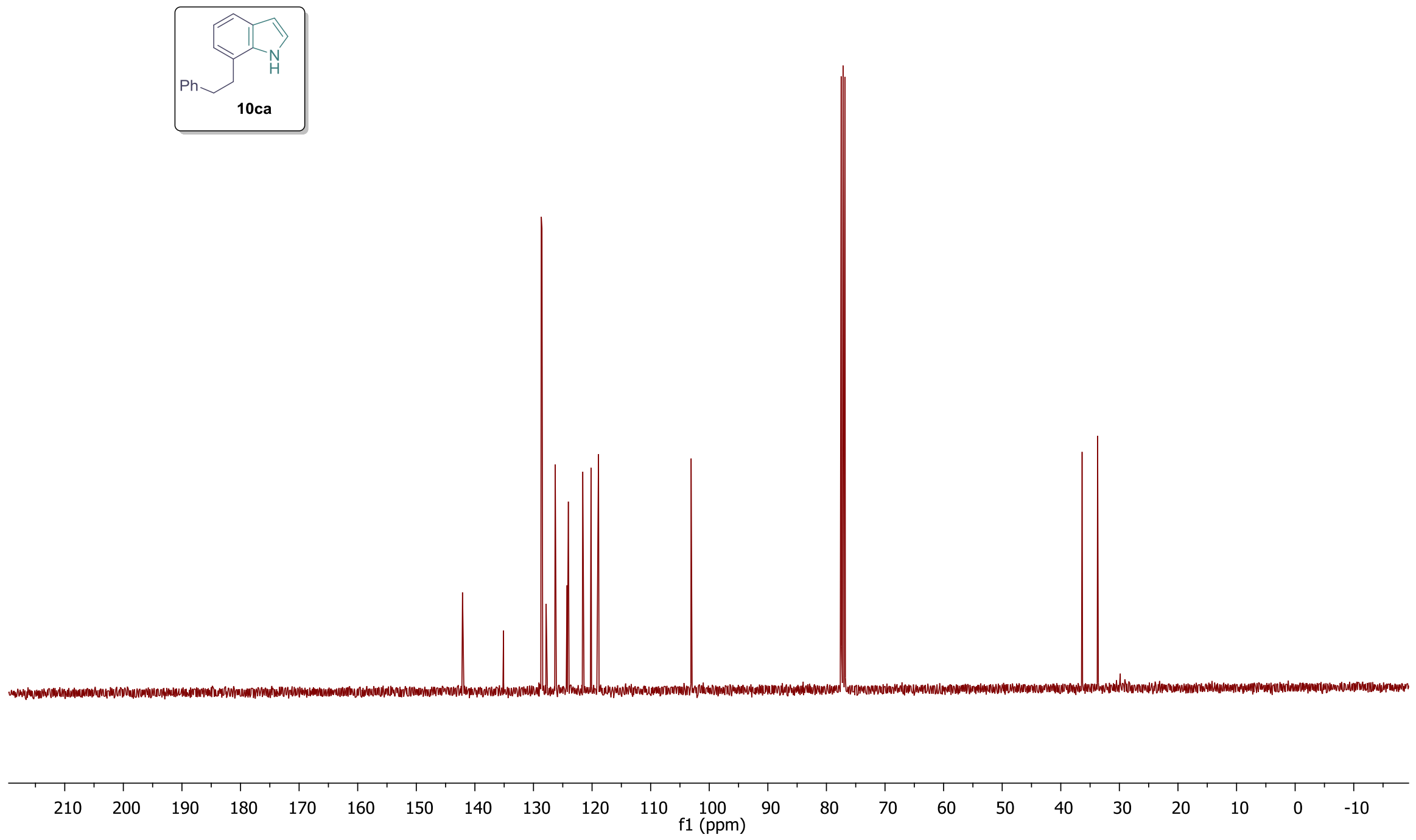

S133 

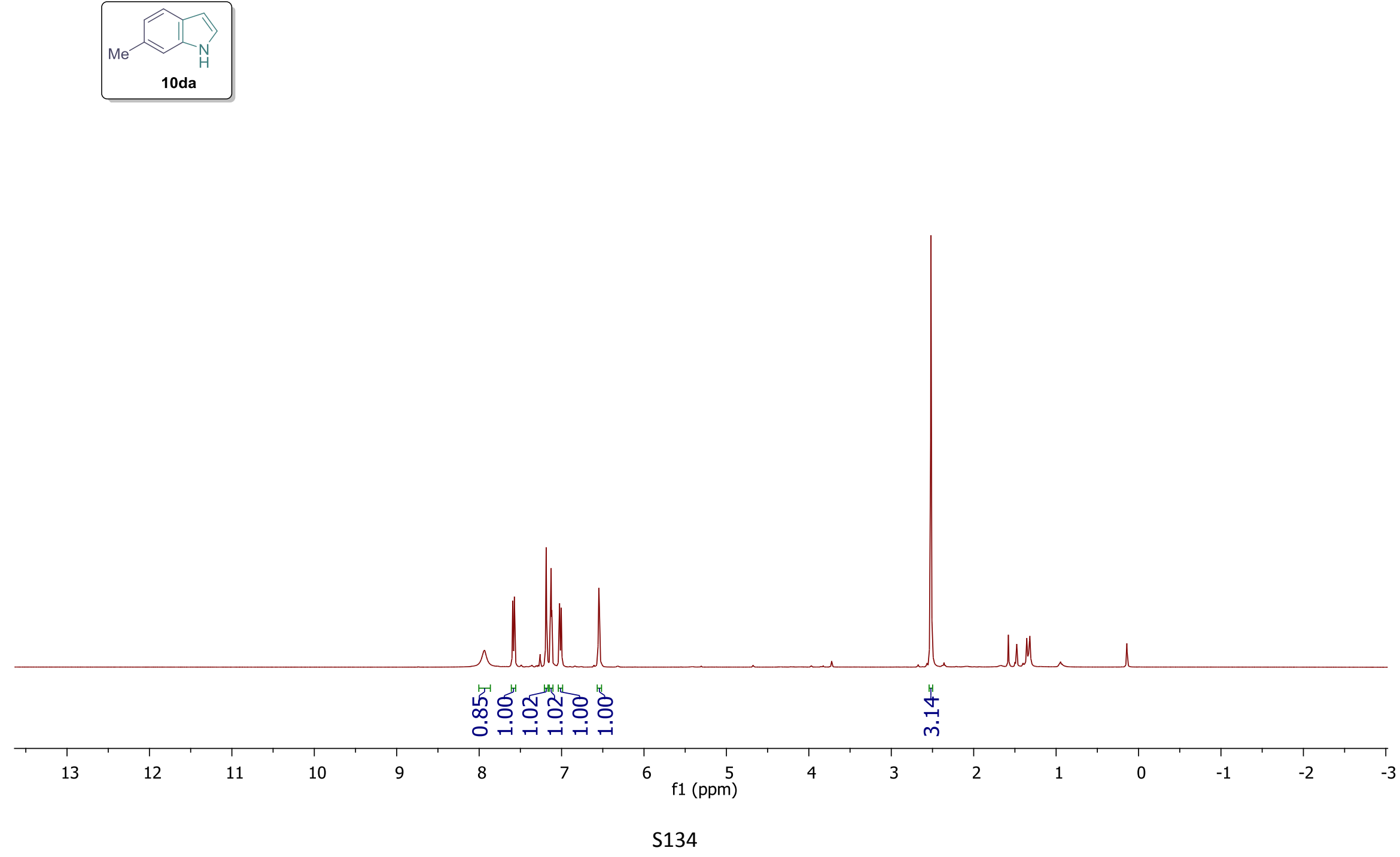


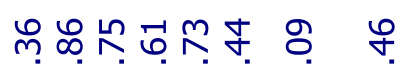

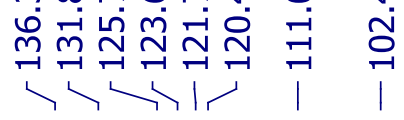
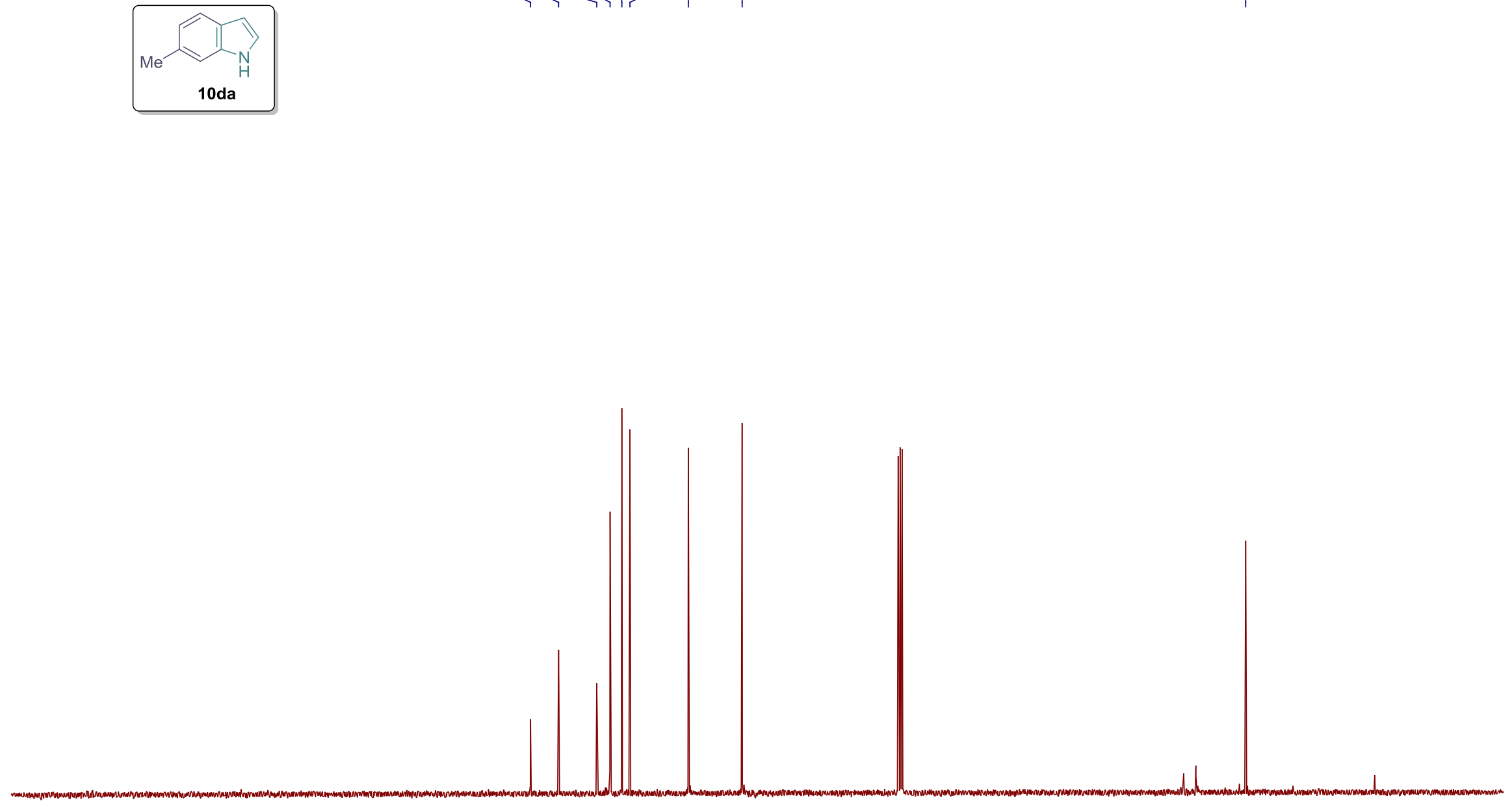


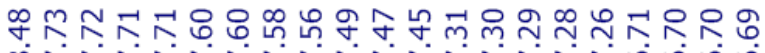

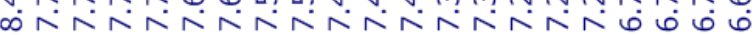

1 1
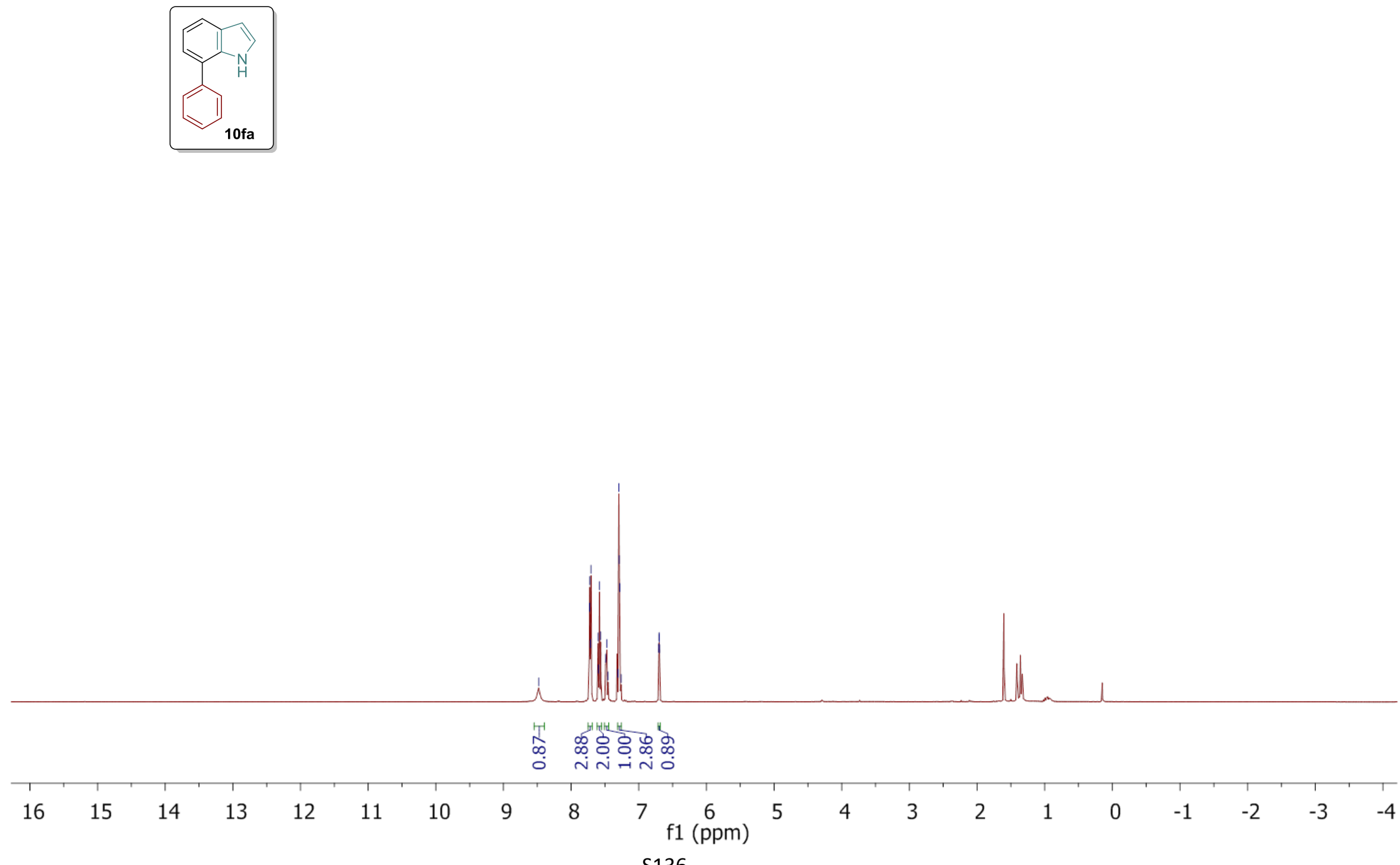


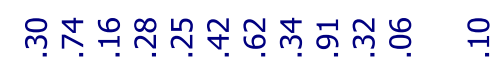

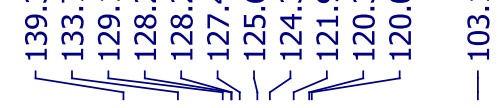
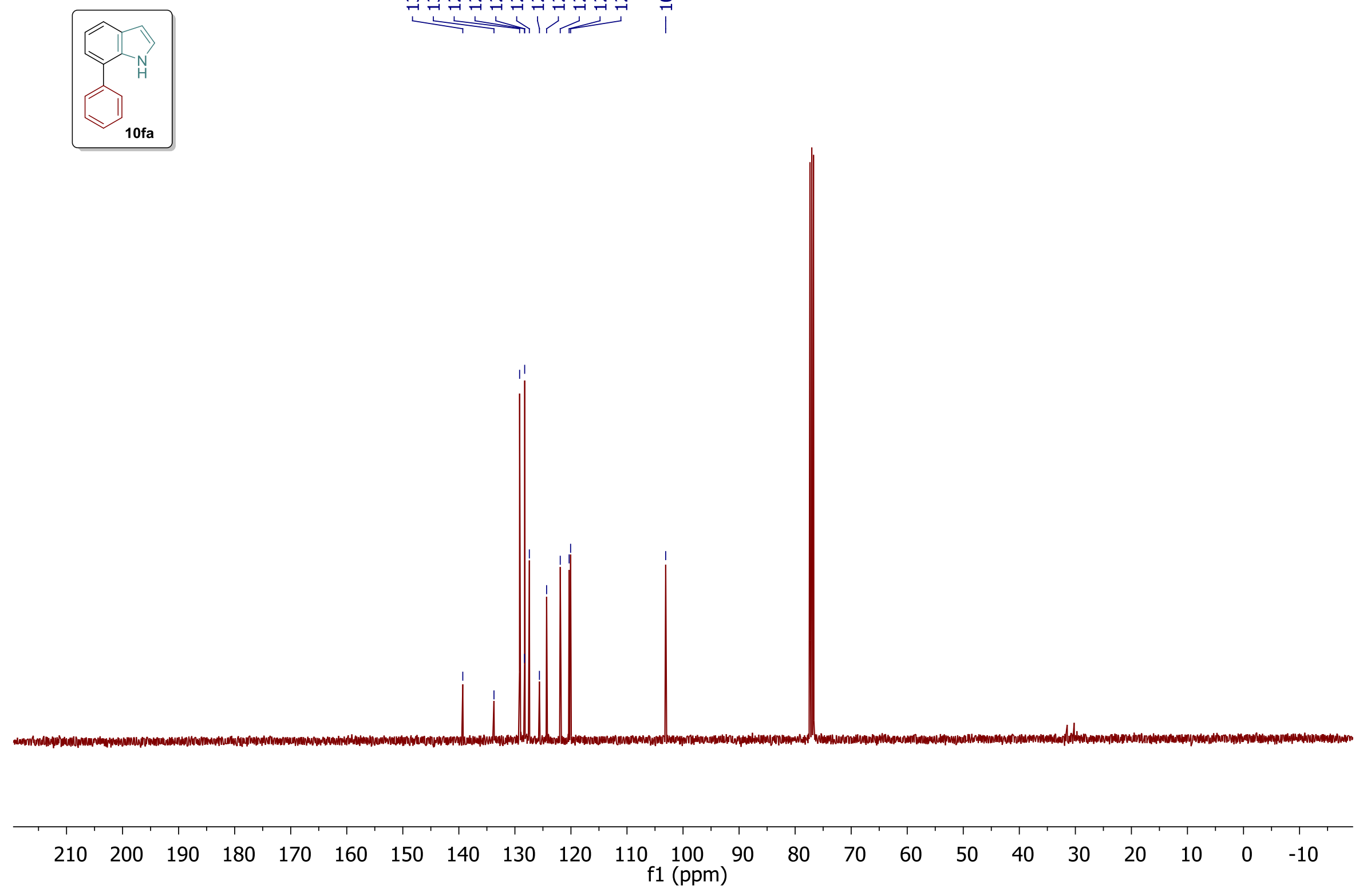

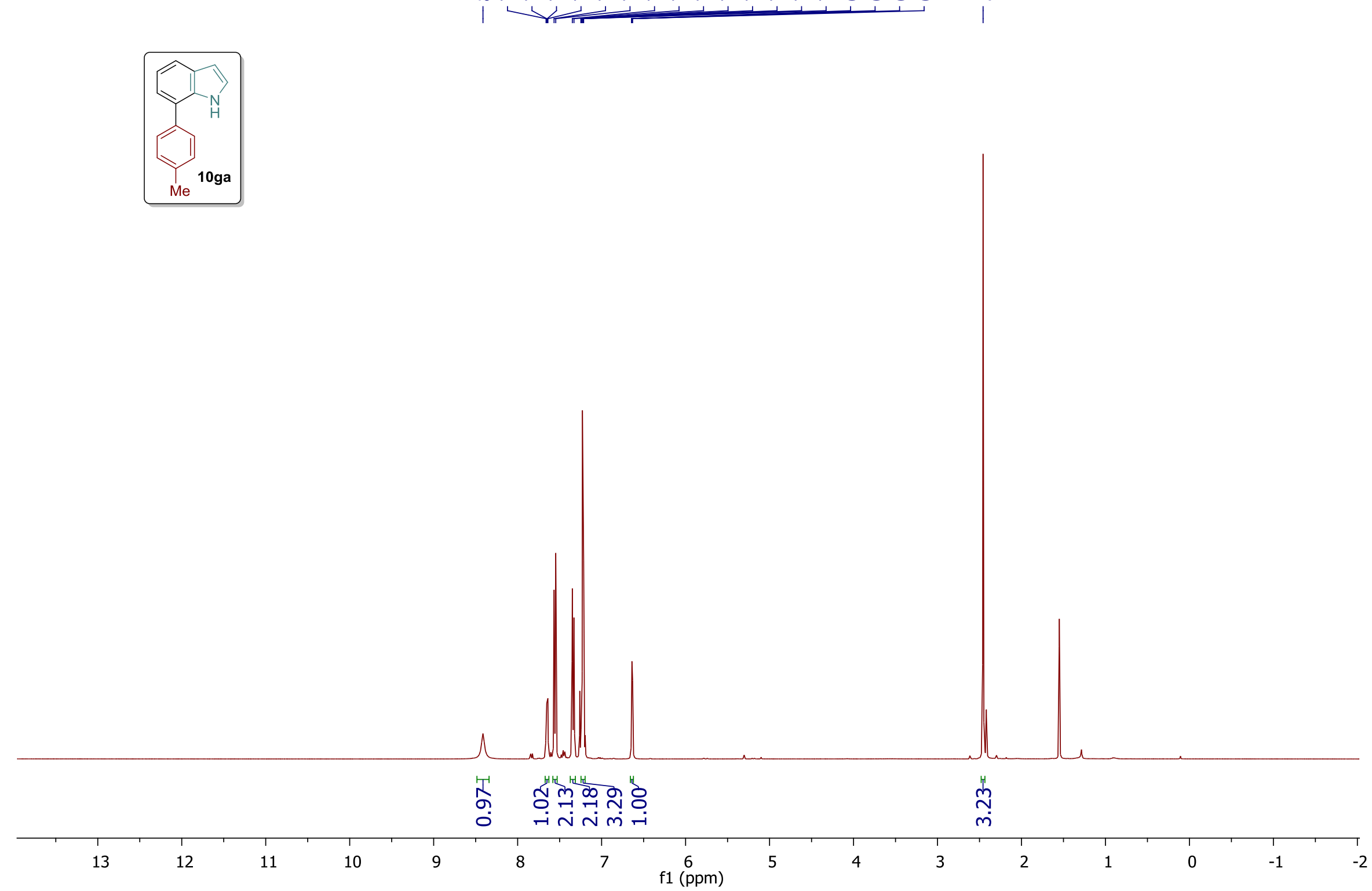


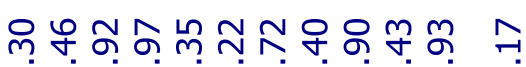

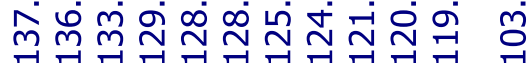
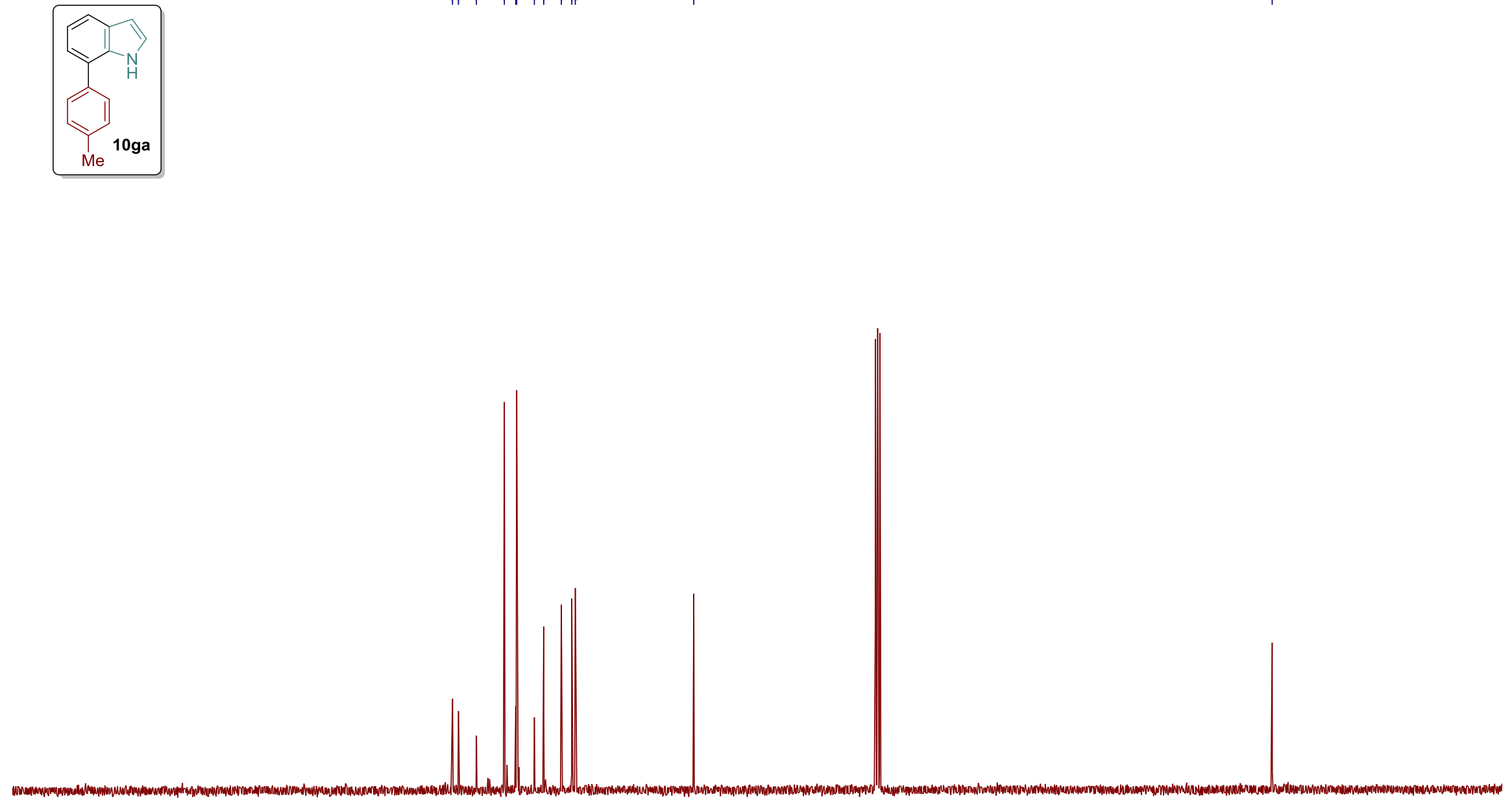


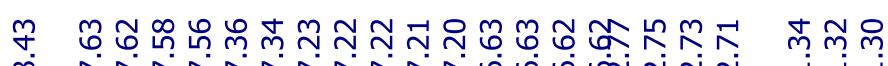

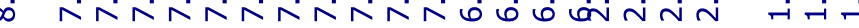
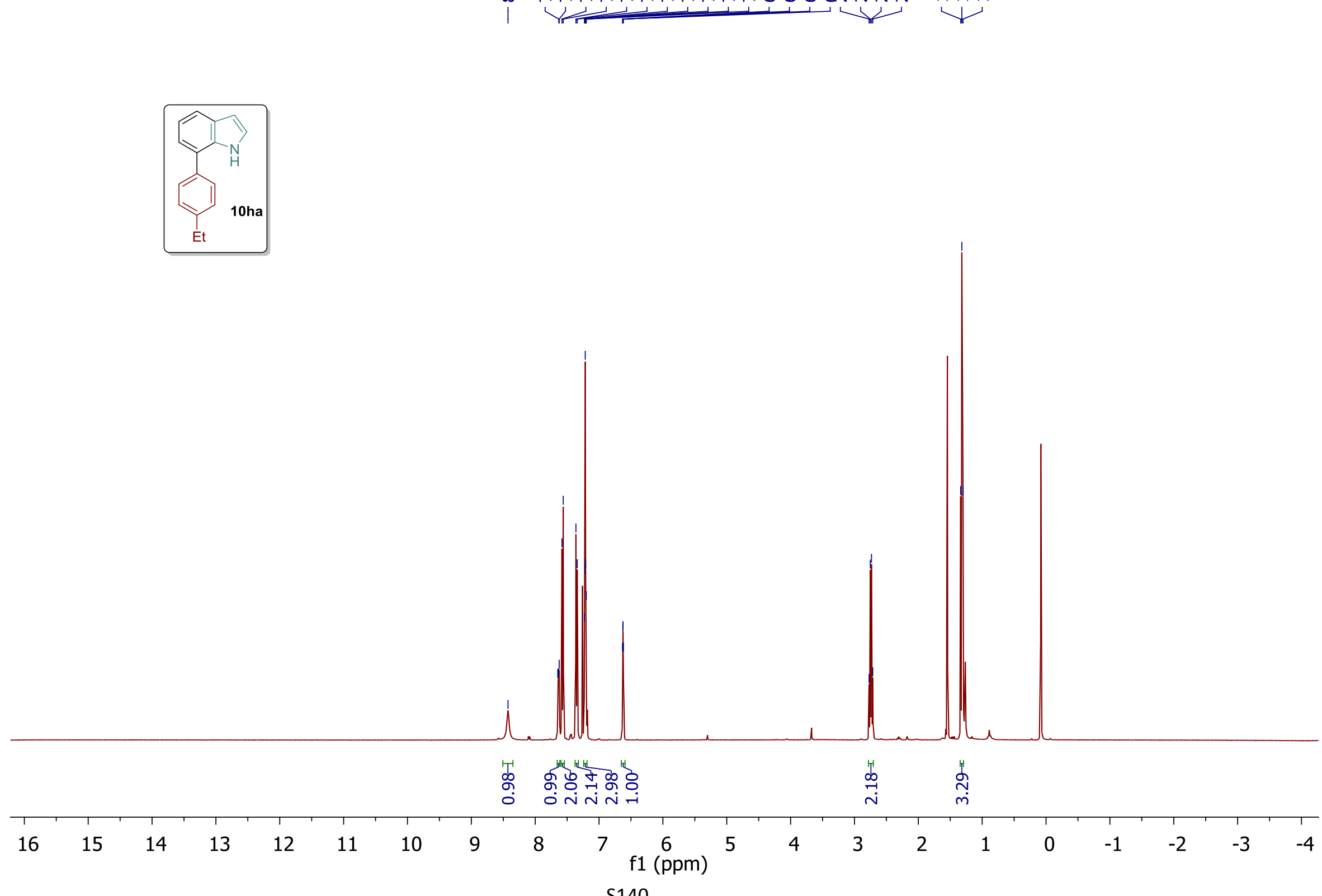


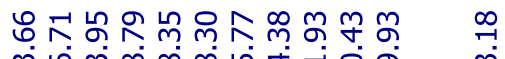

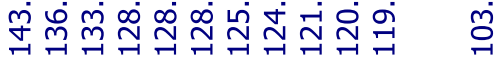

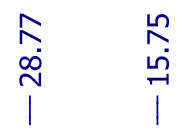
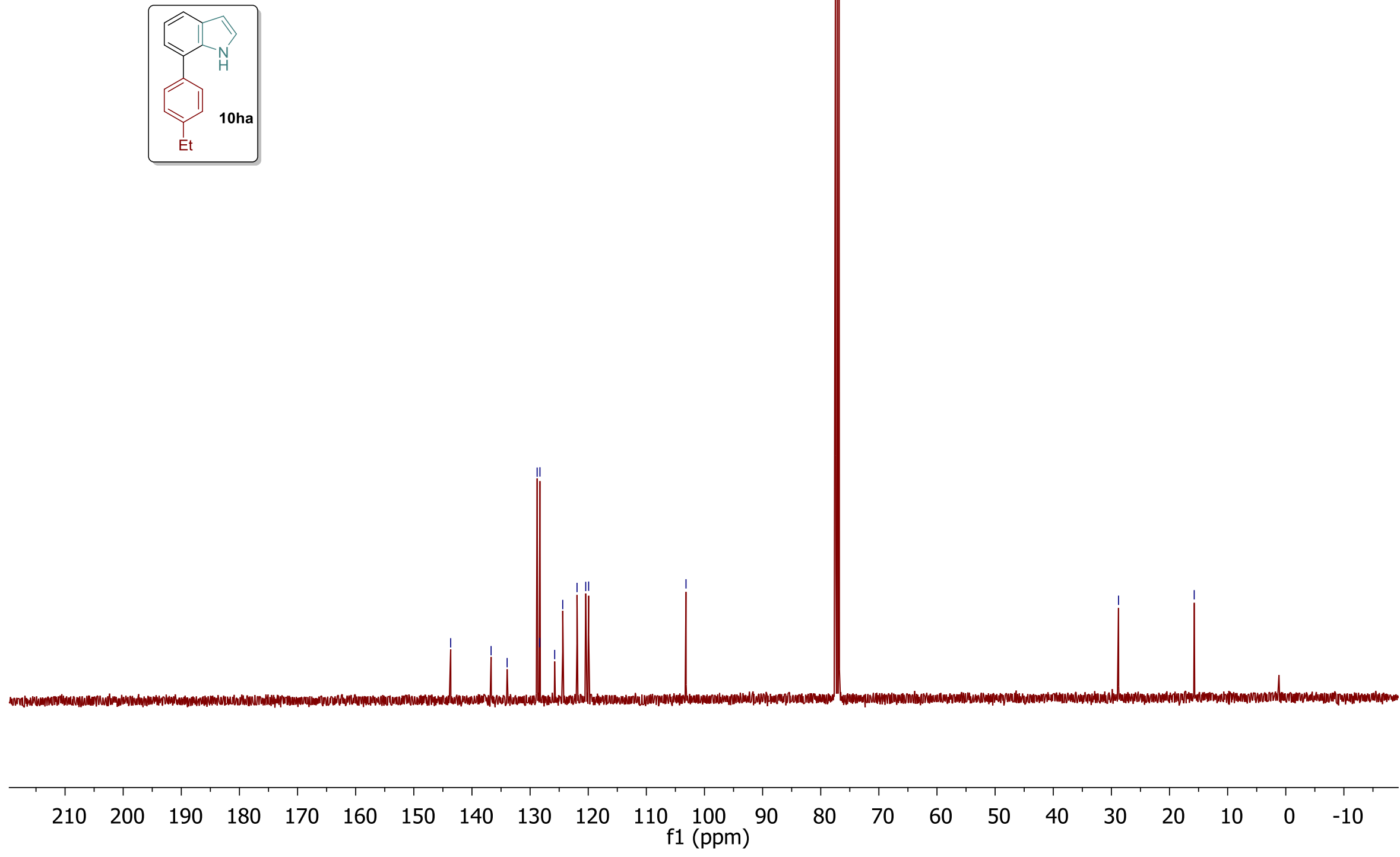


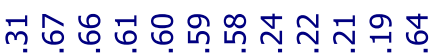

m

L

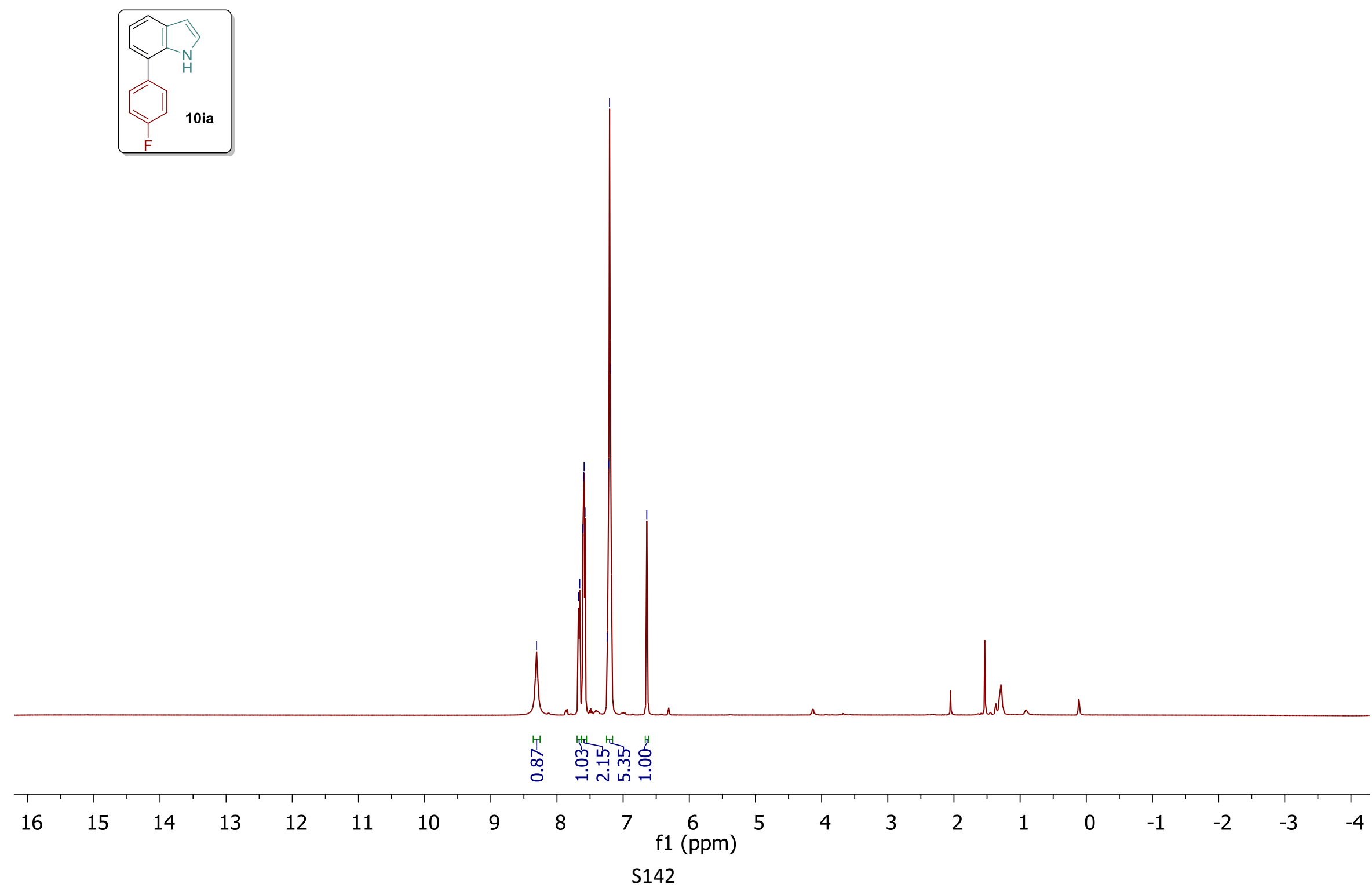



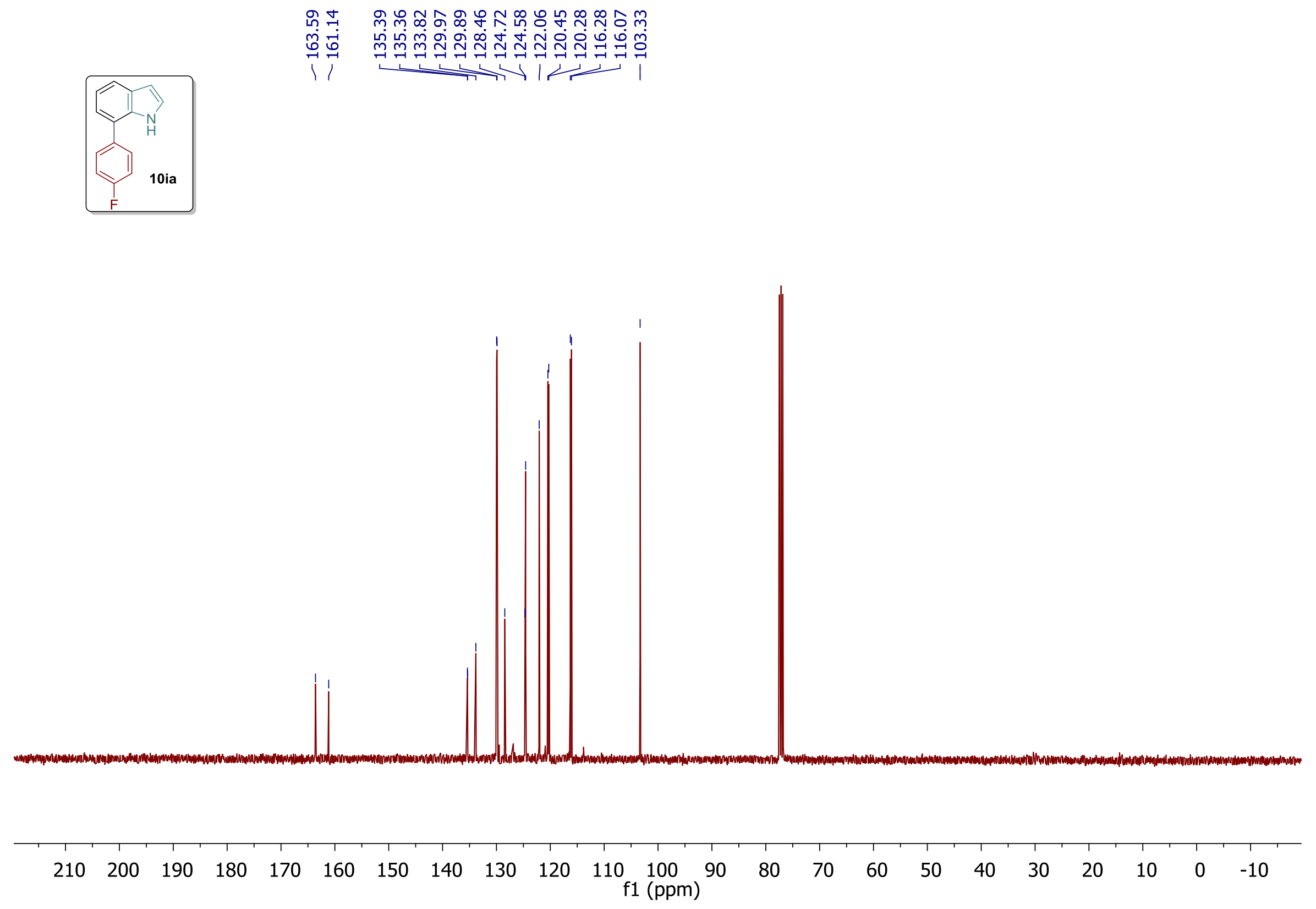
S143 


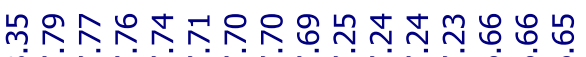

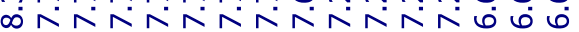

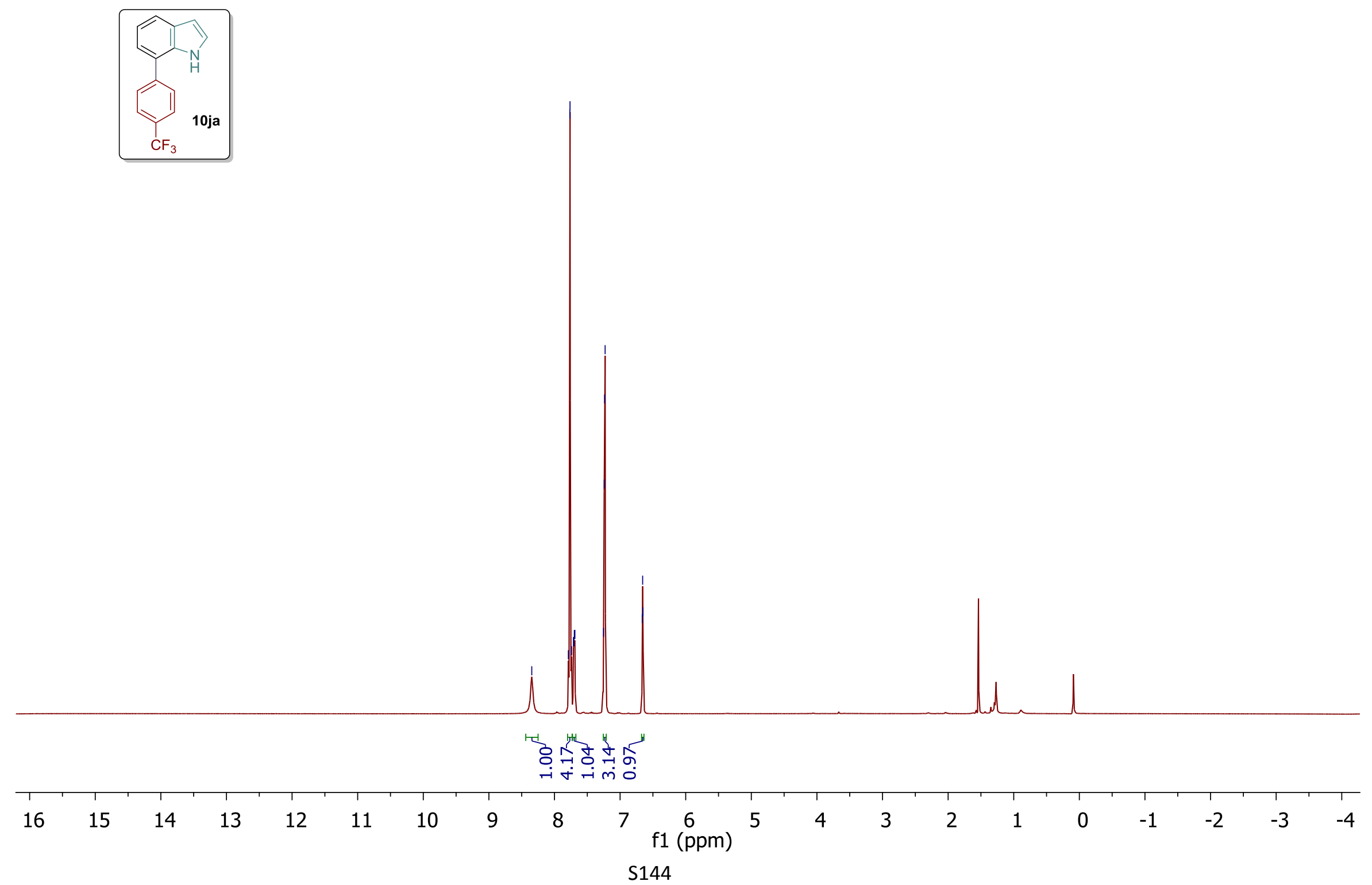



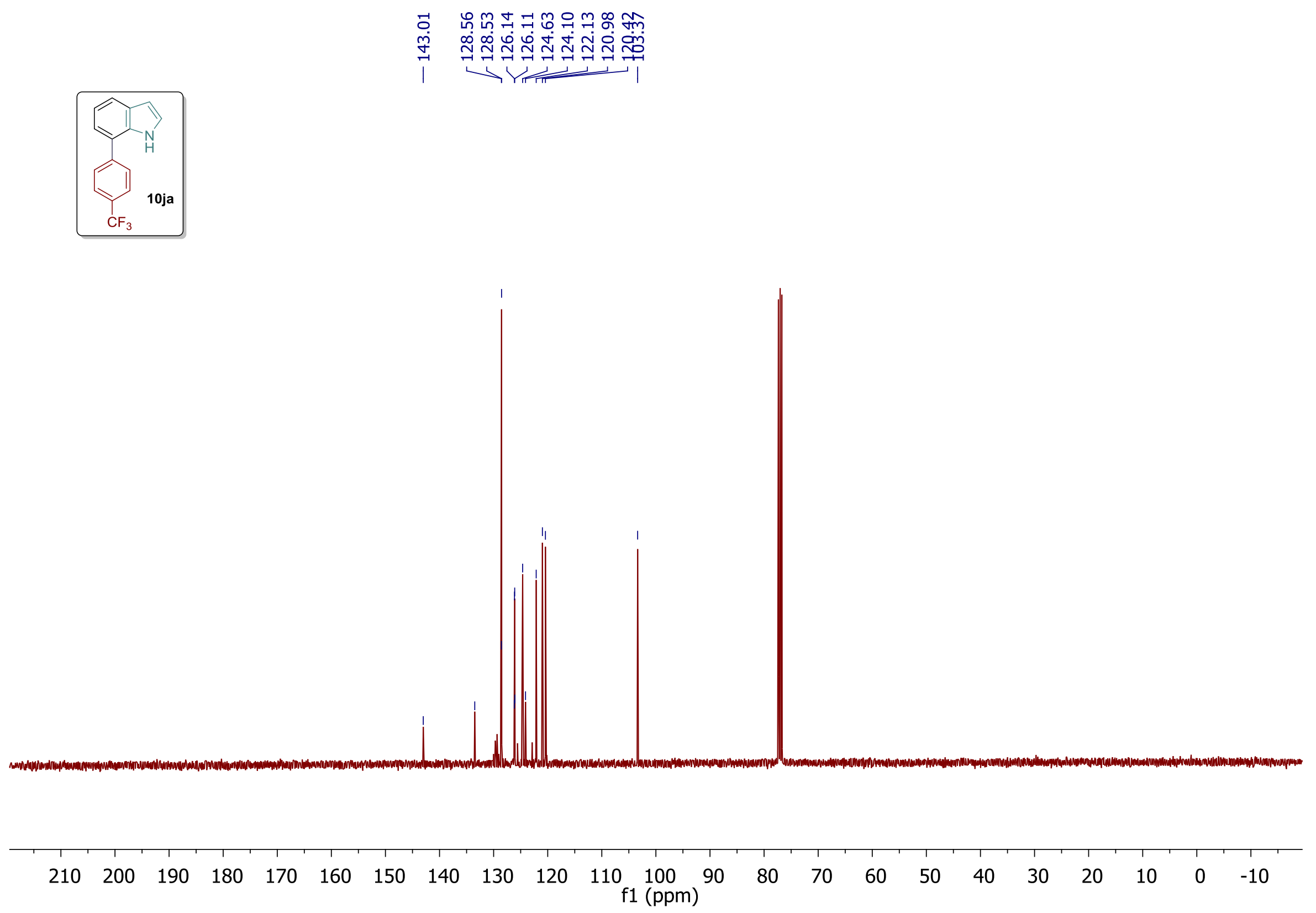
S145 


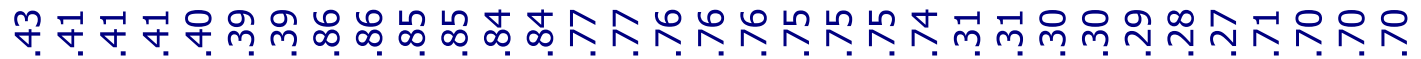

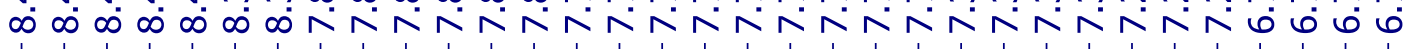
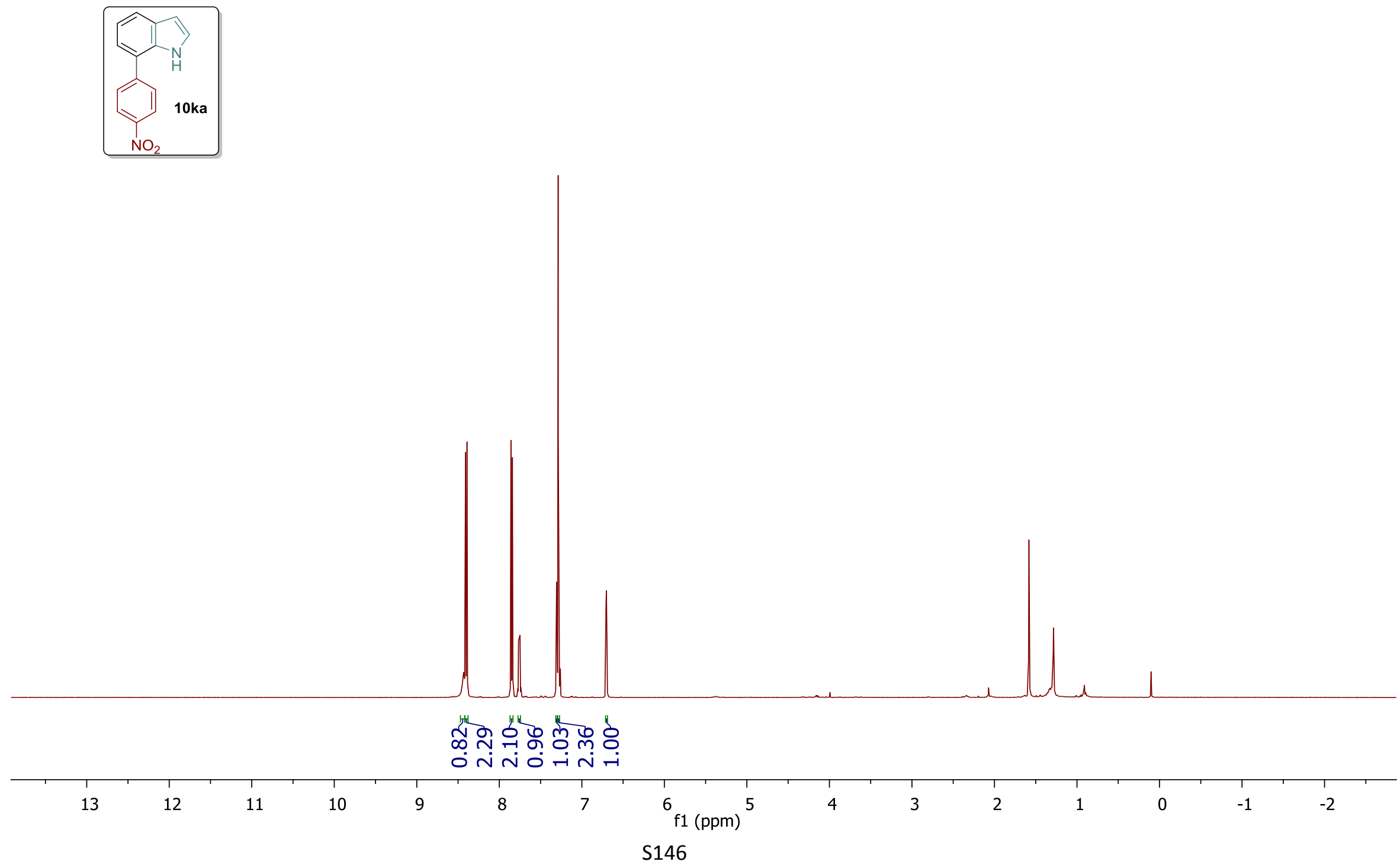


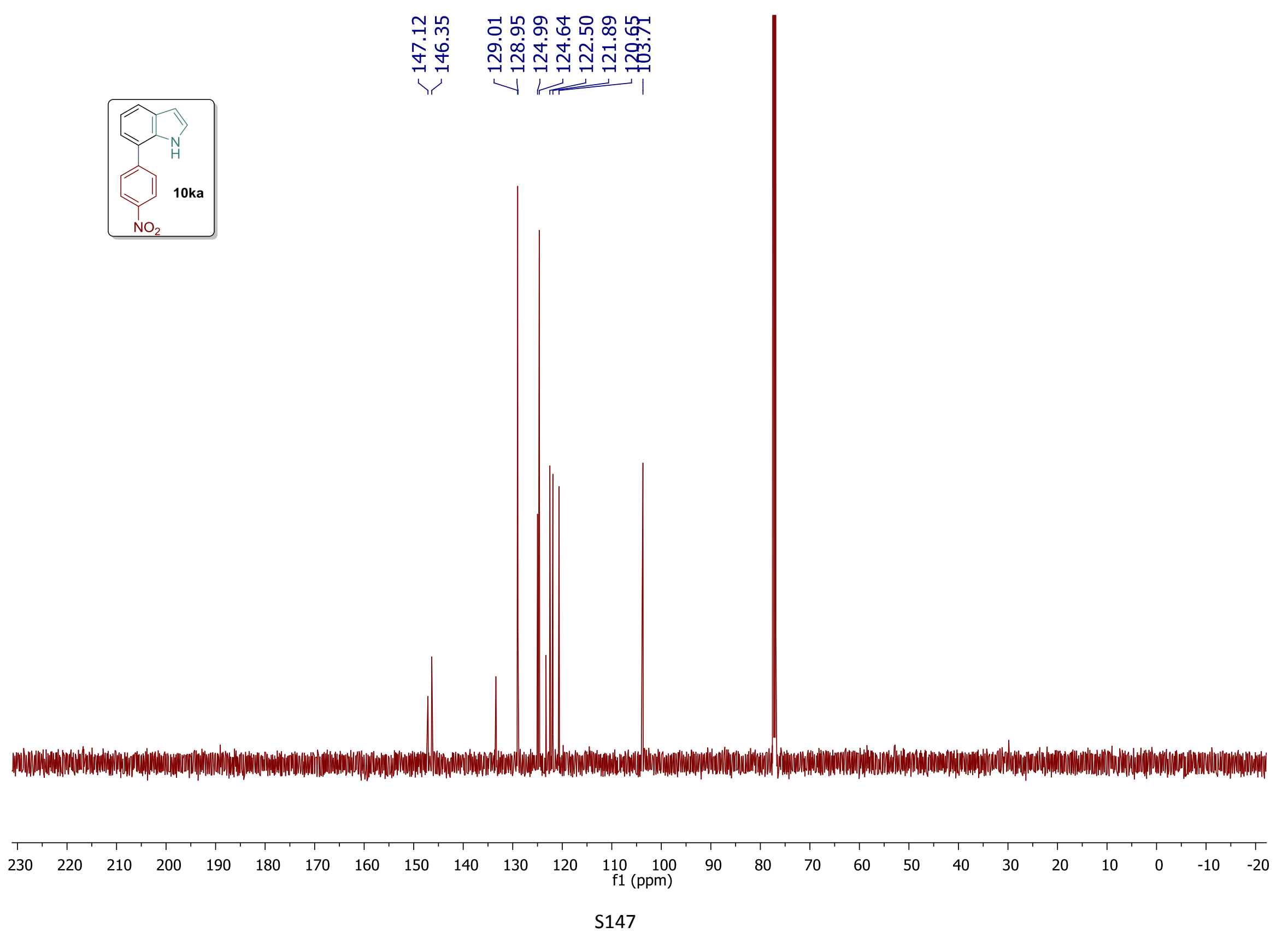




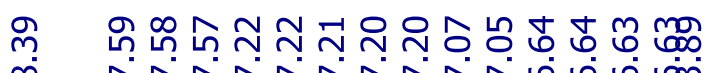

क

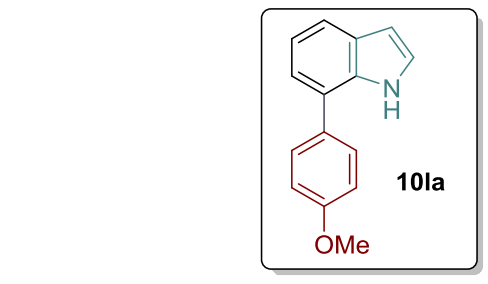

促

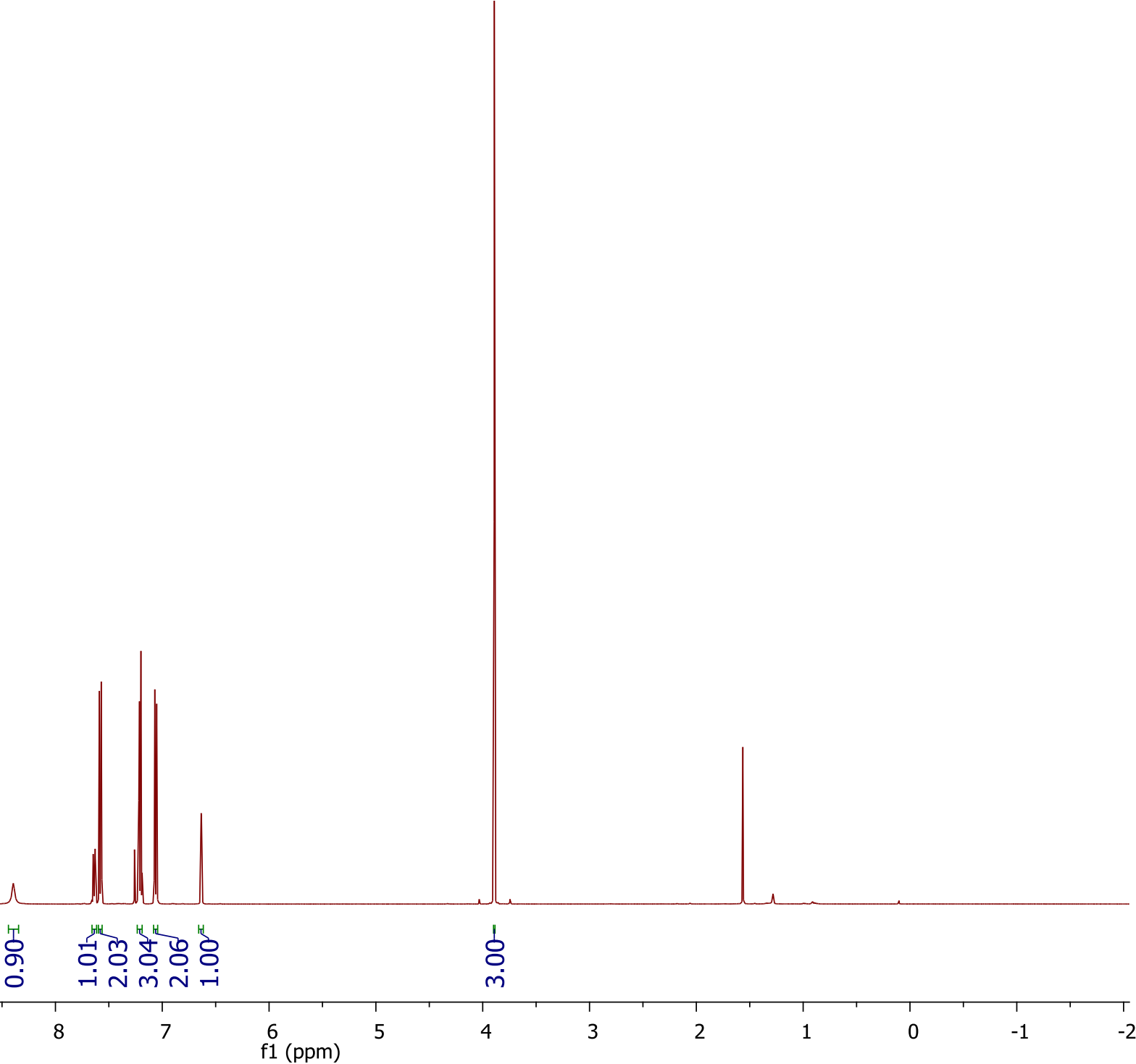




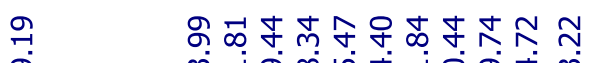

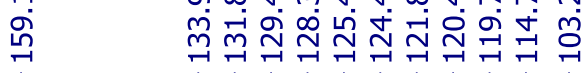

另

证
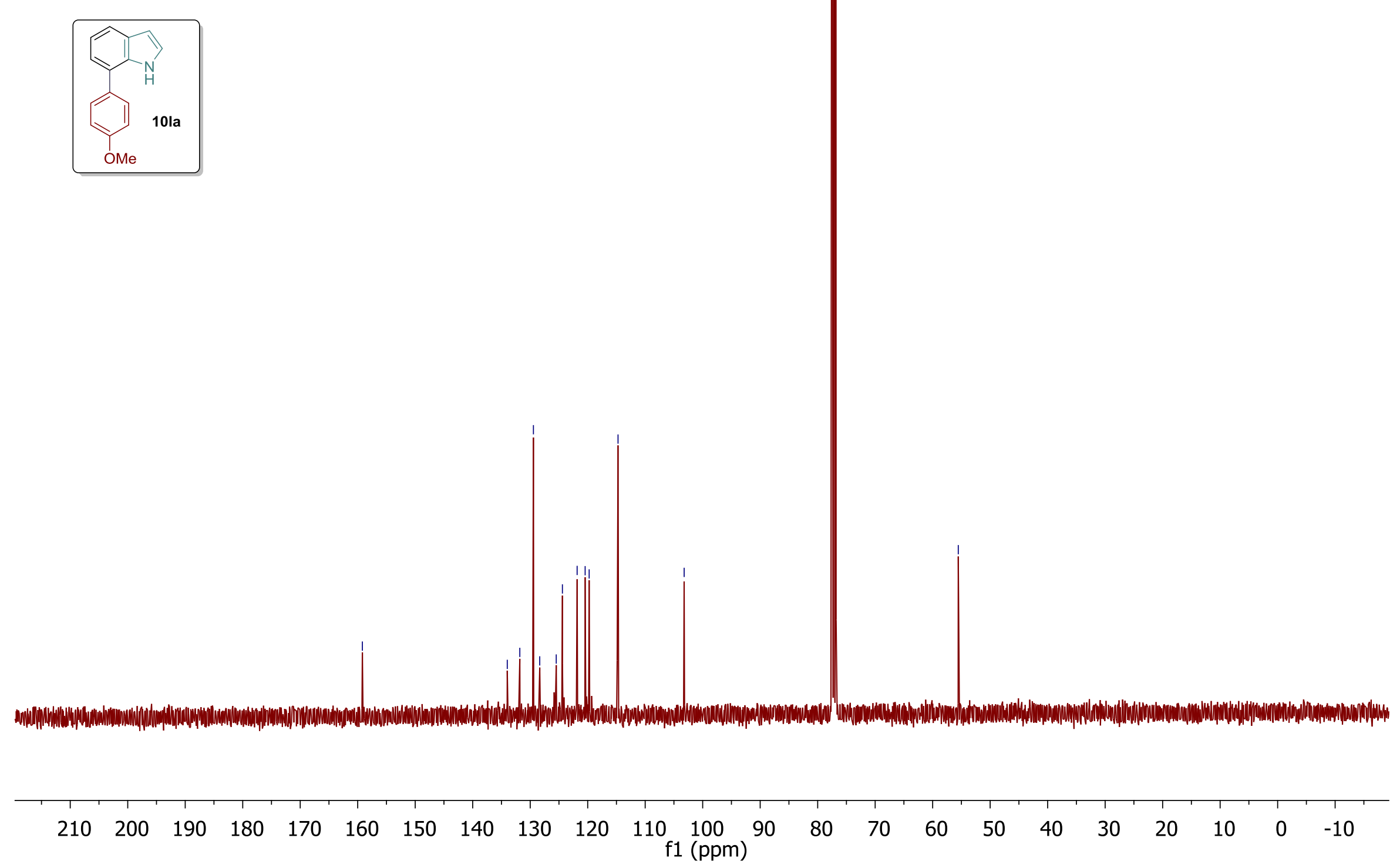

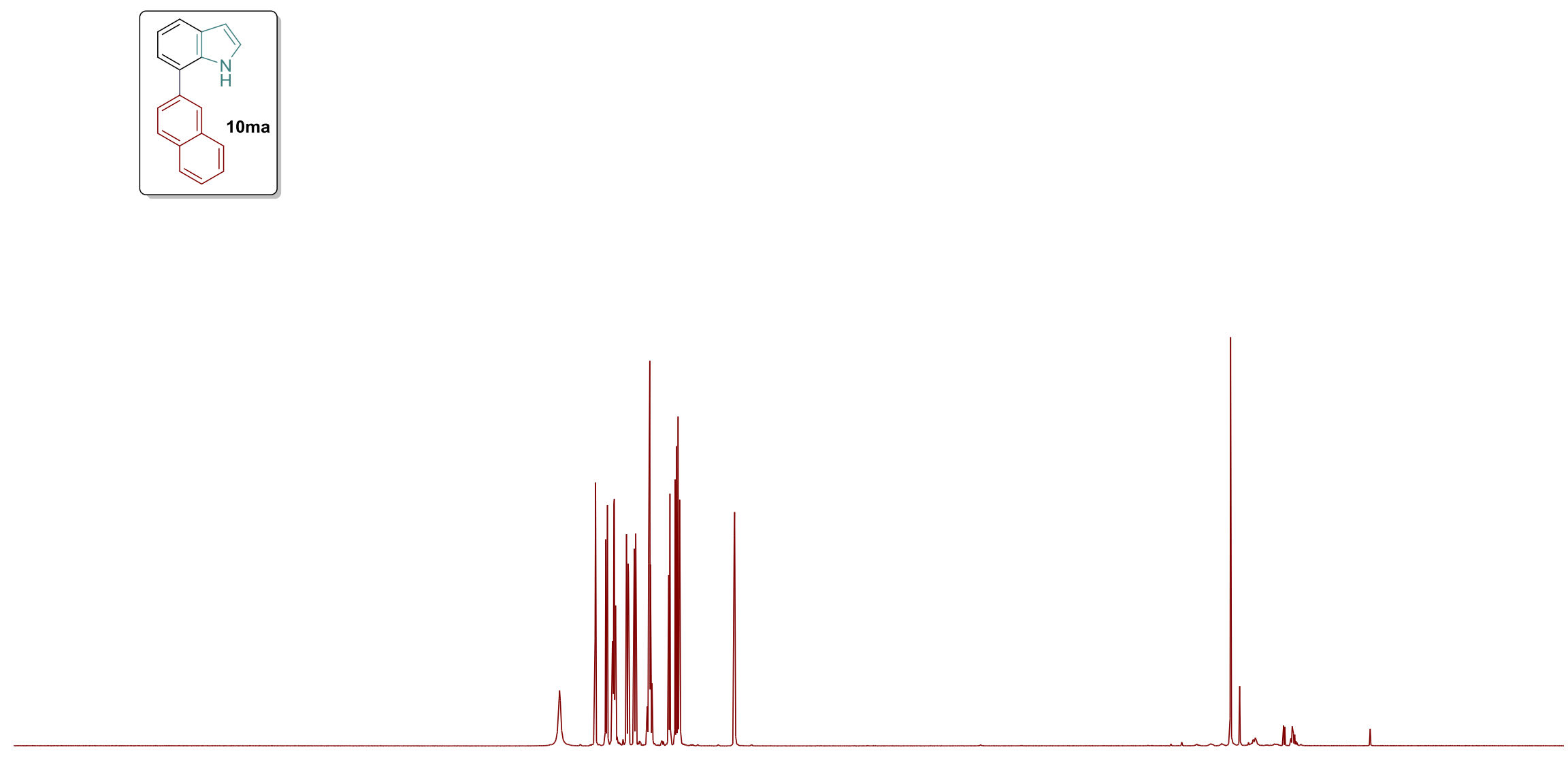

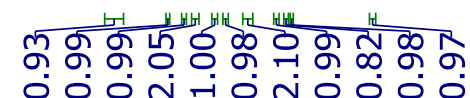

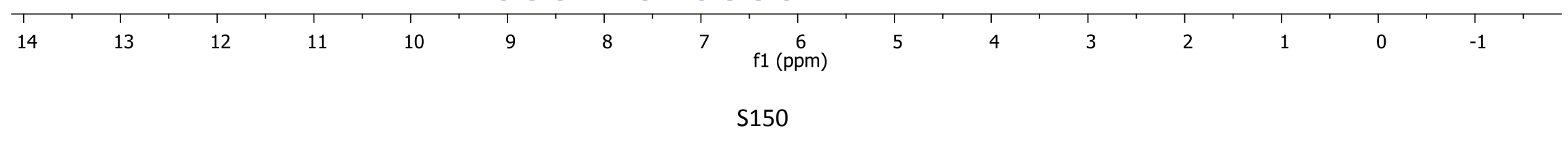




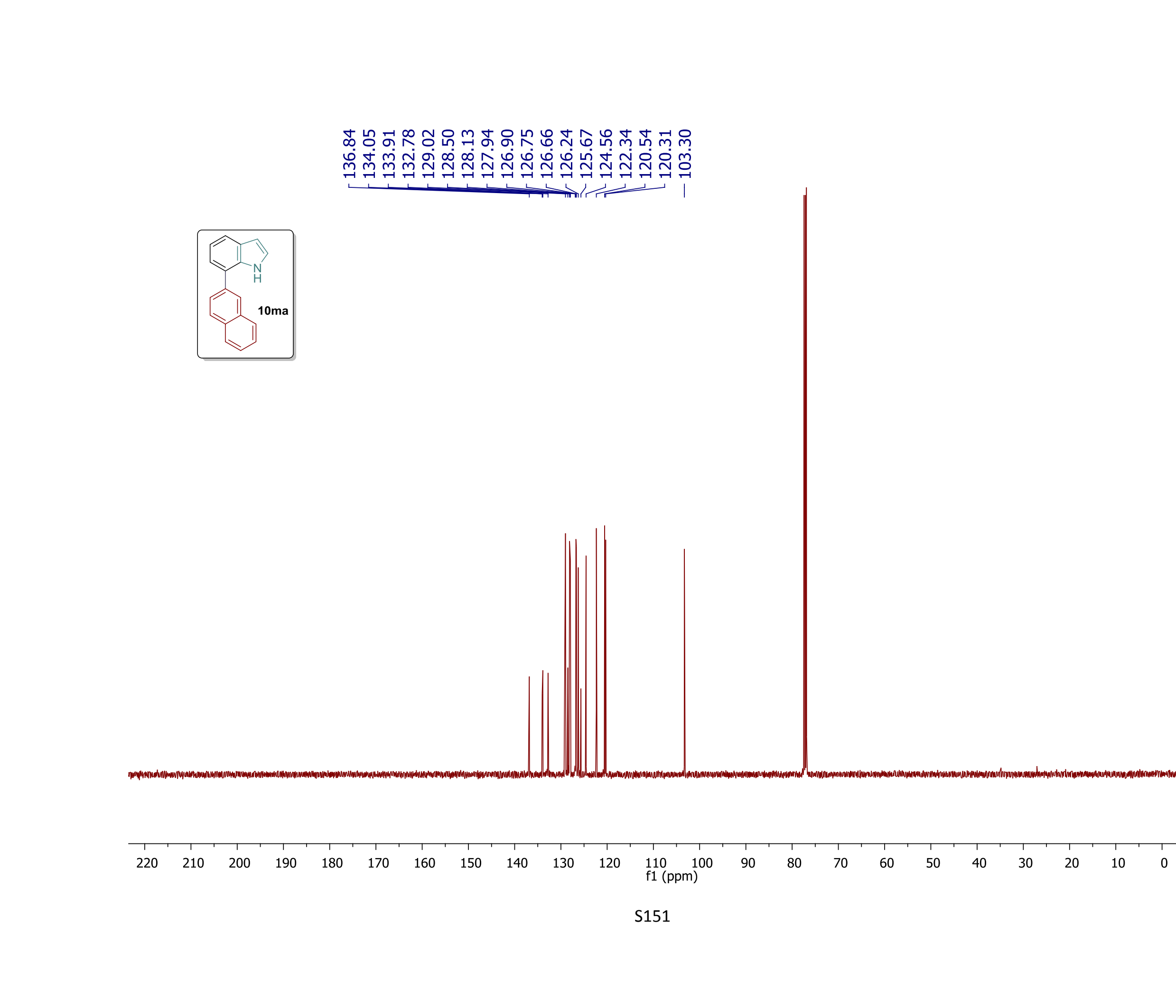




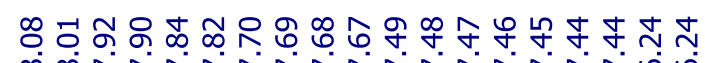

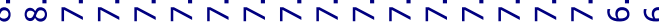

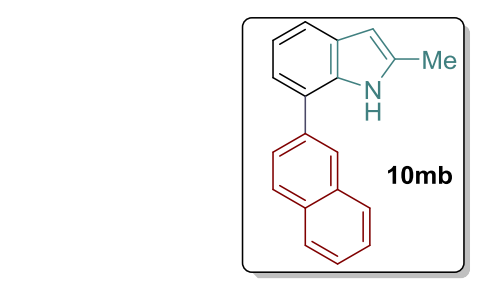

I

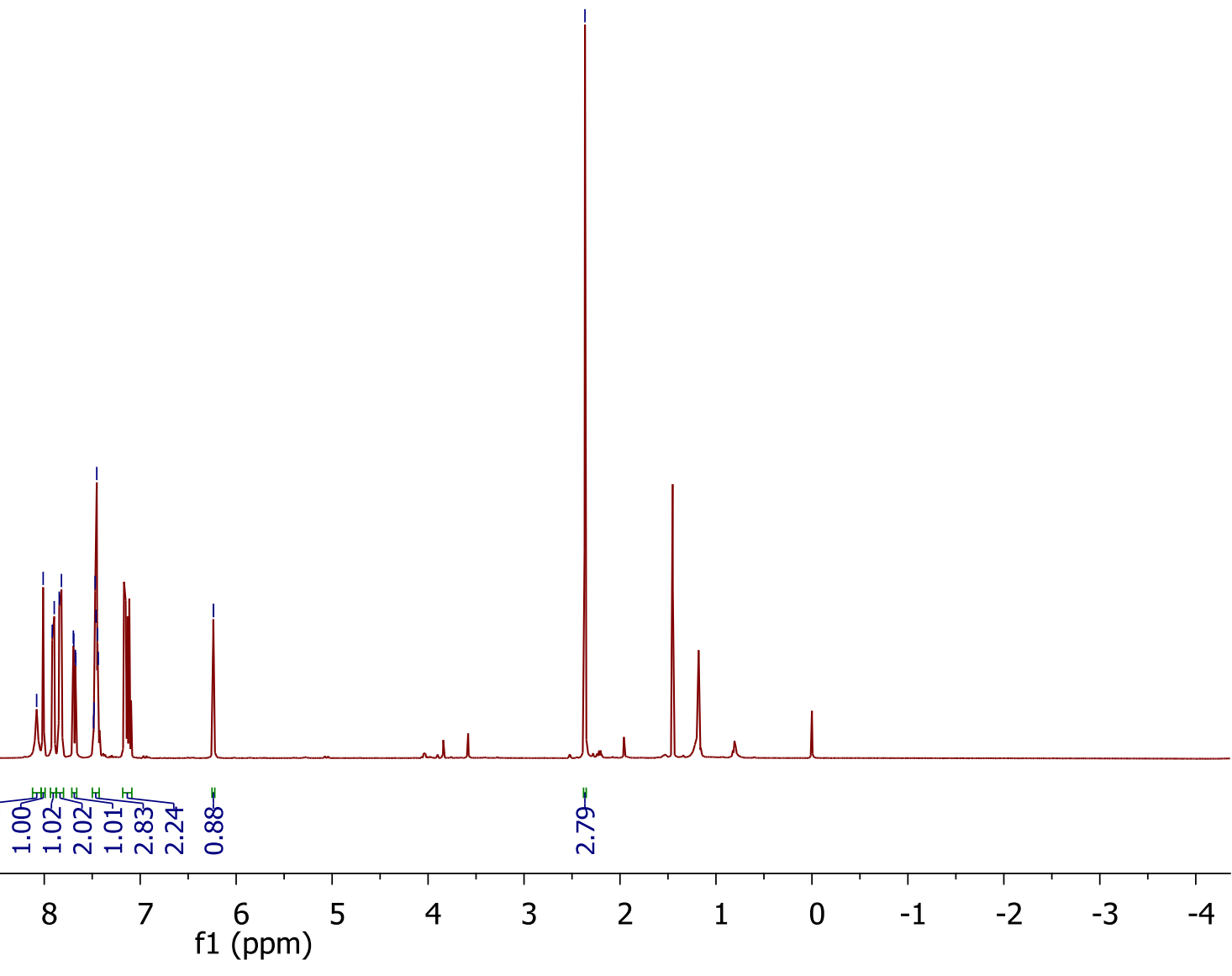




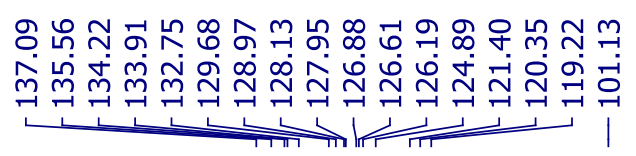

ผू กั
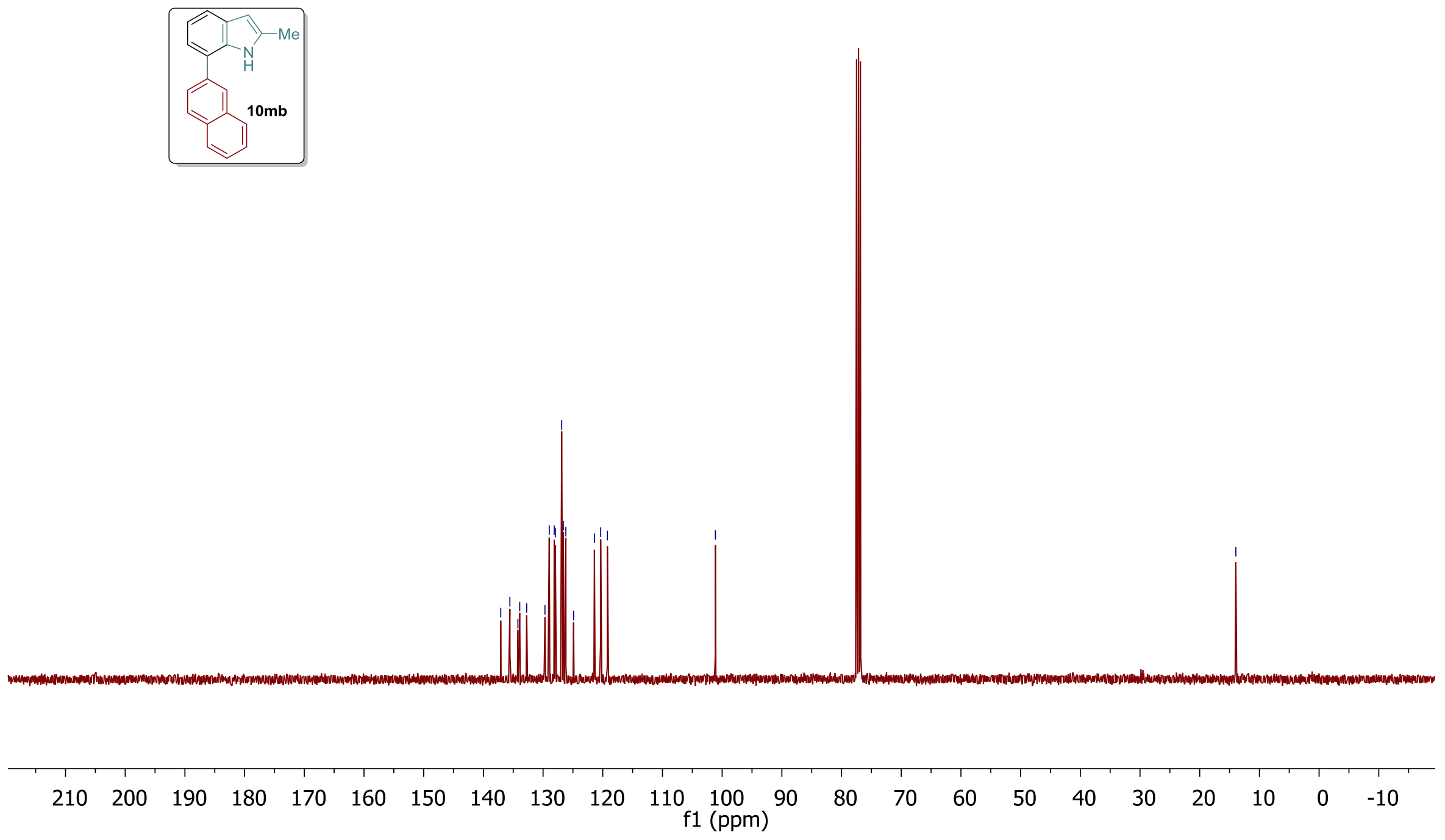

S153 


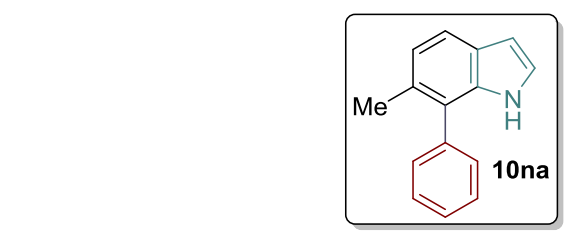

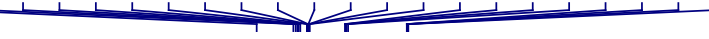

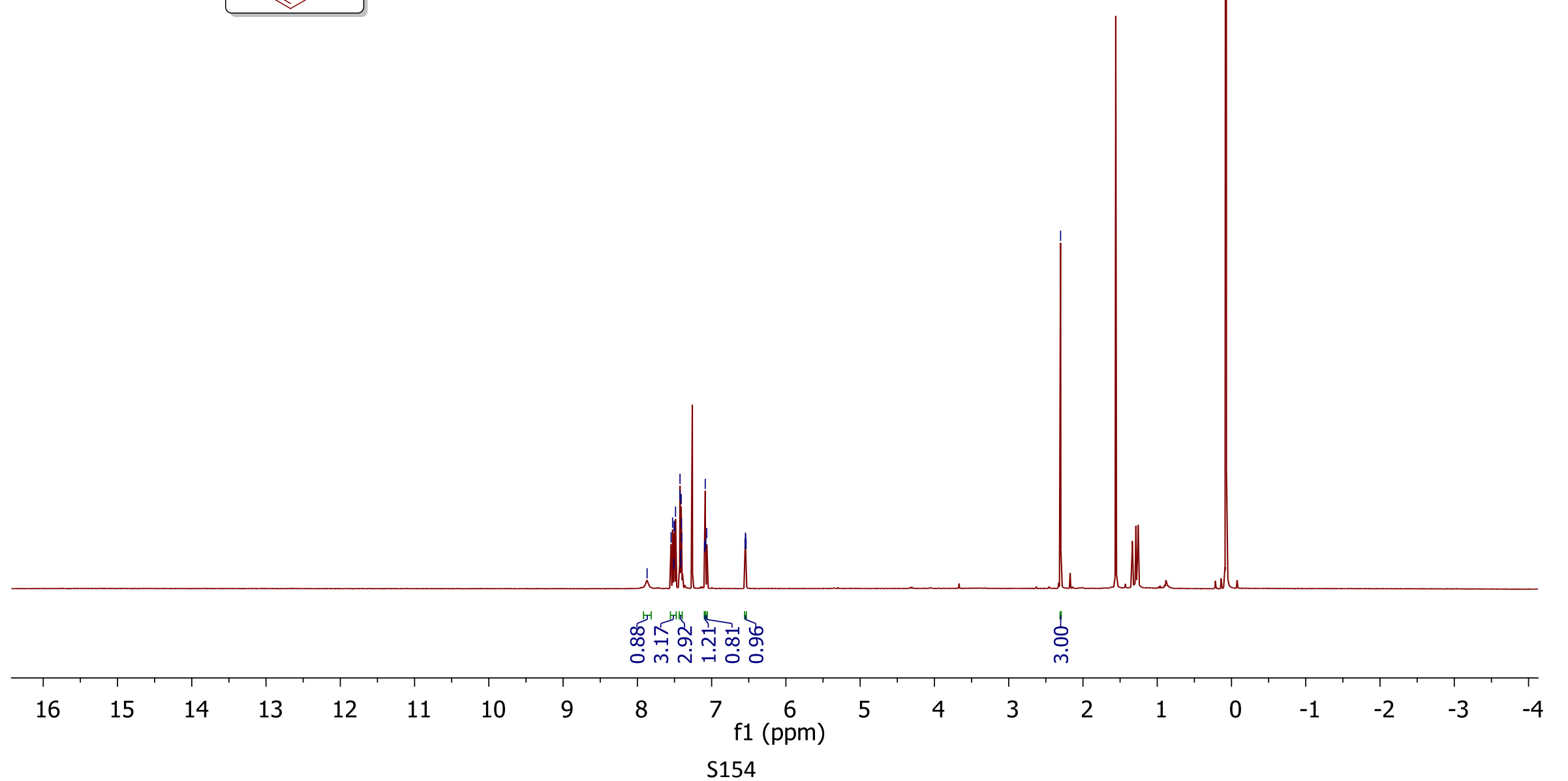




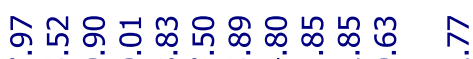

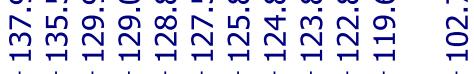

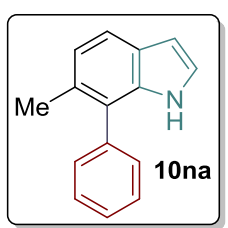

।
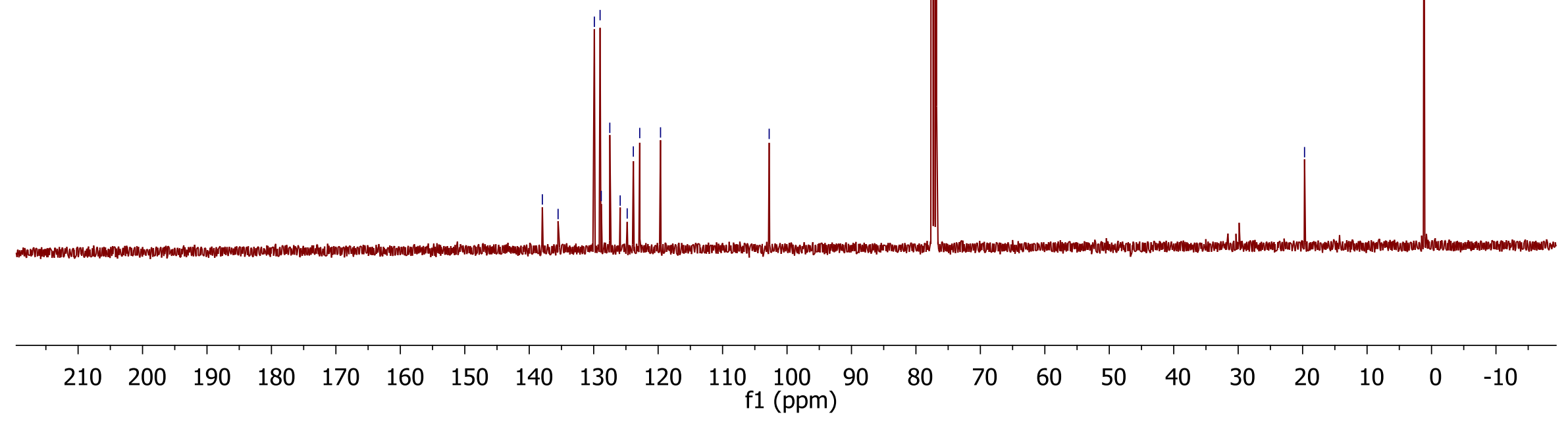

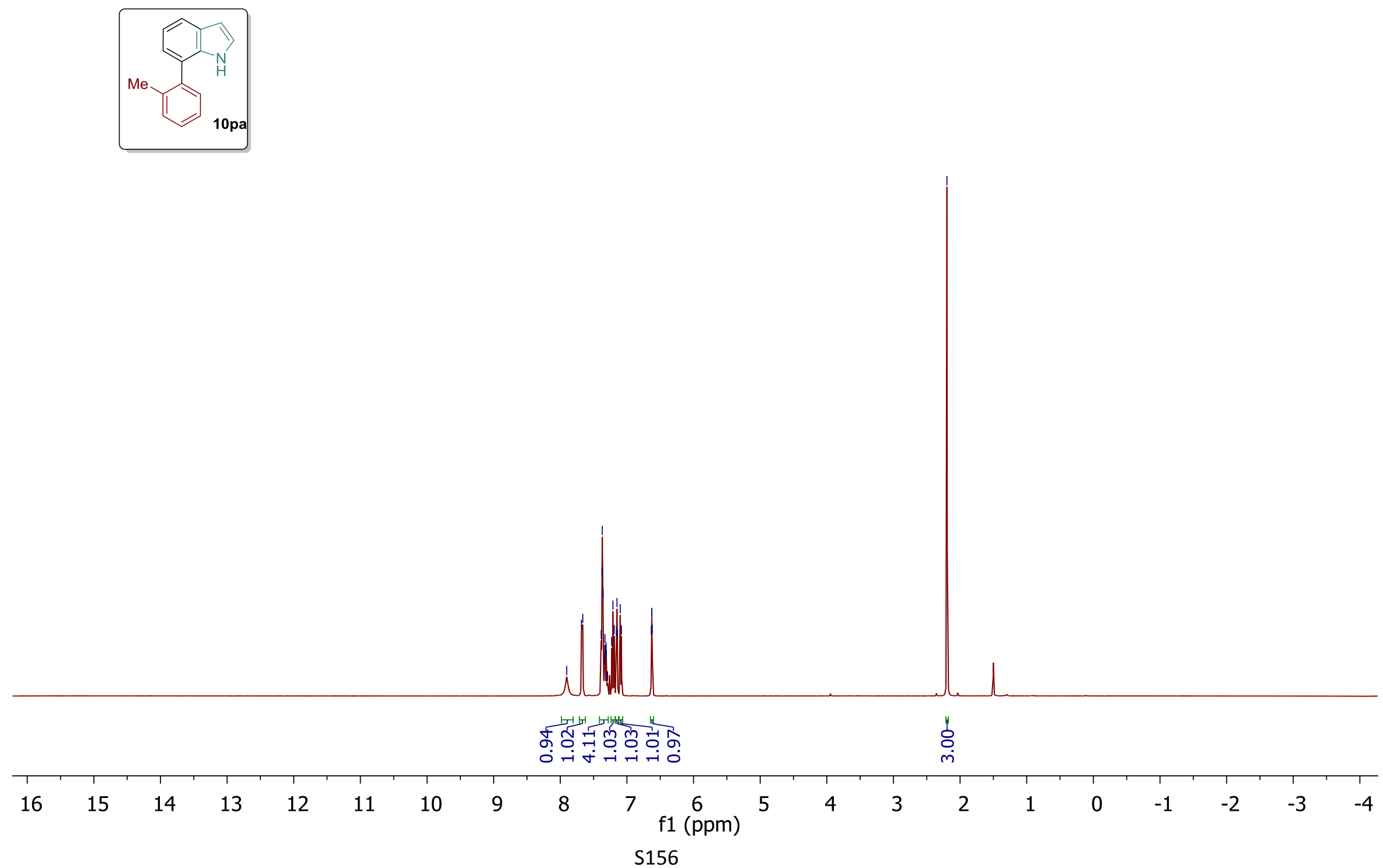


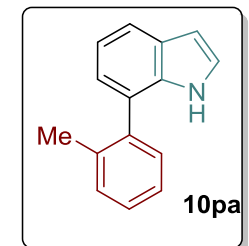

1
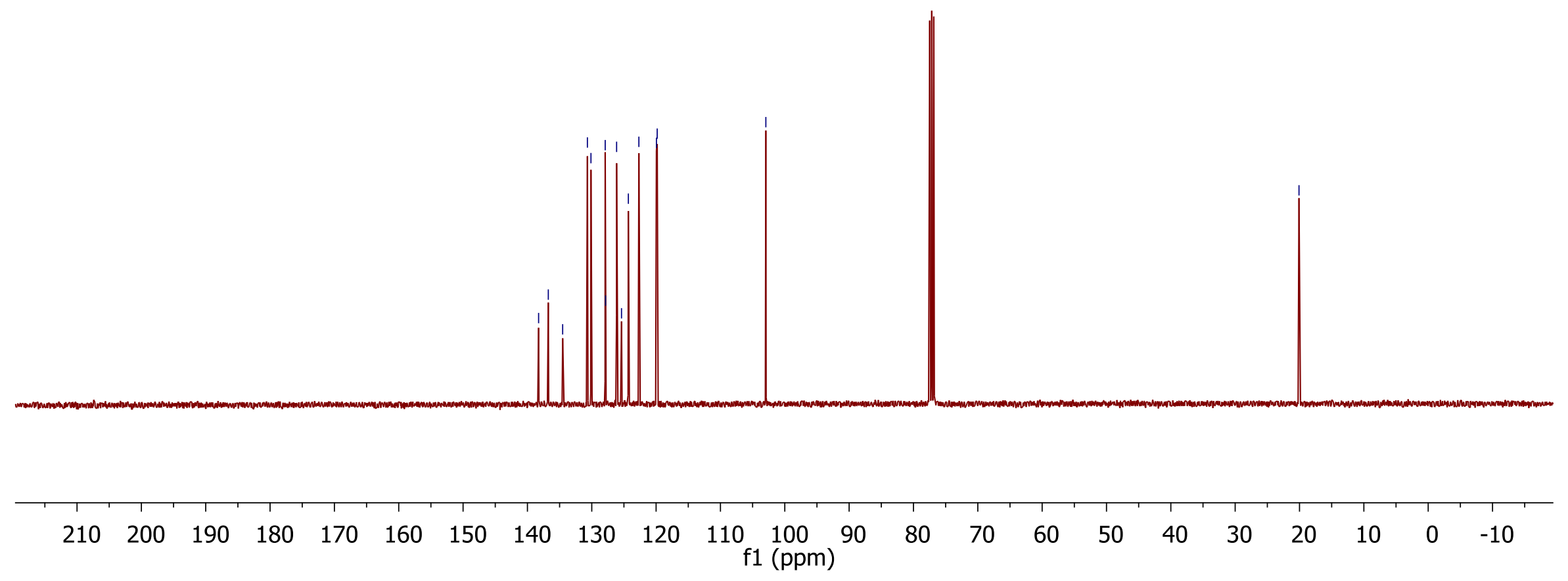


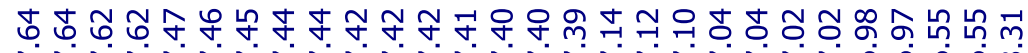

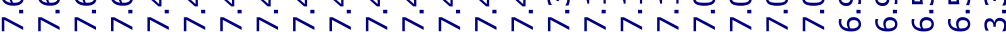

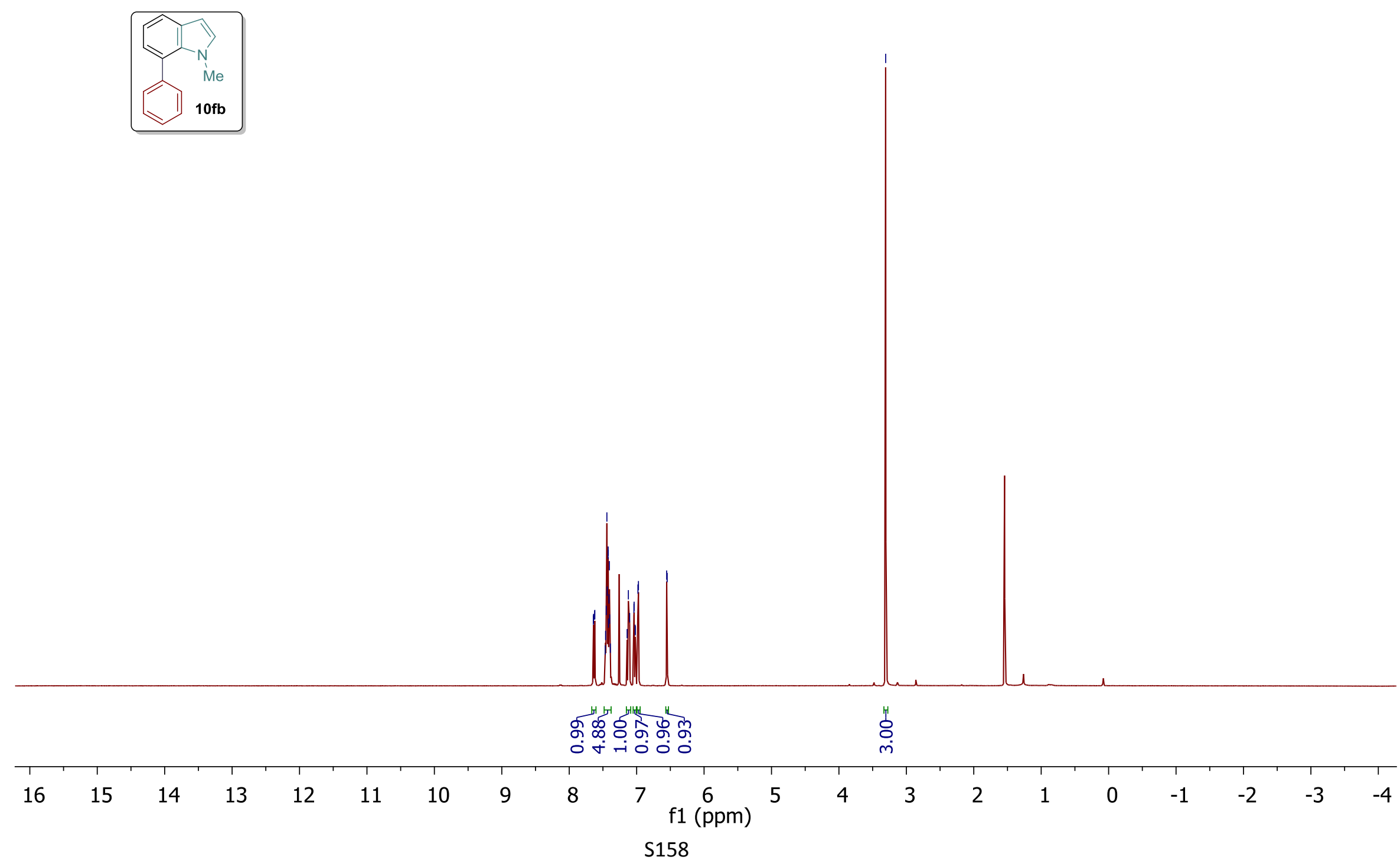



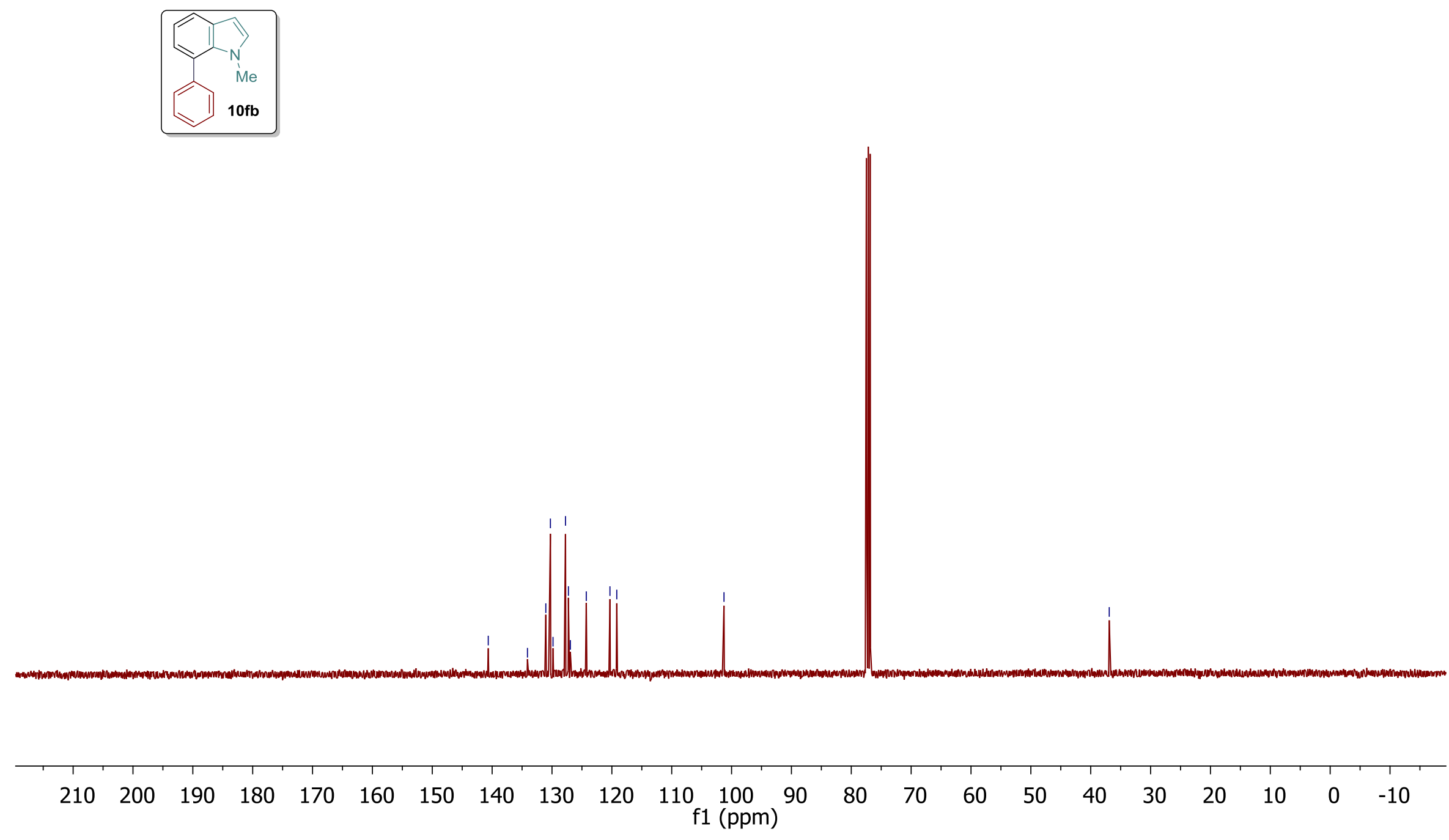


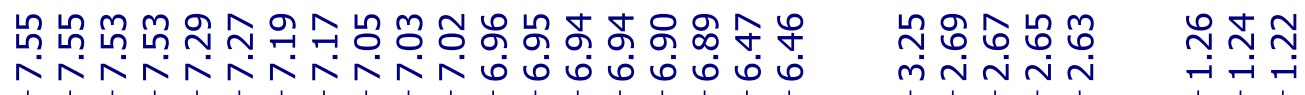

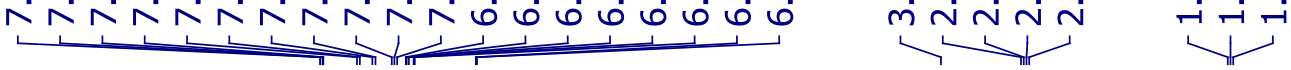
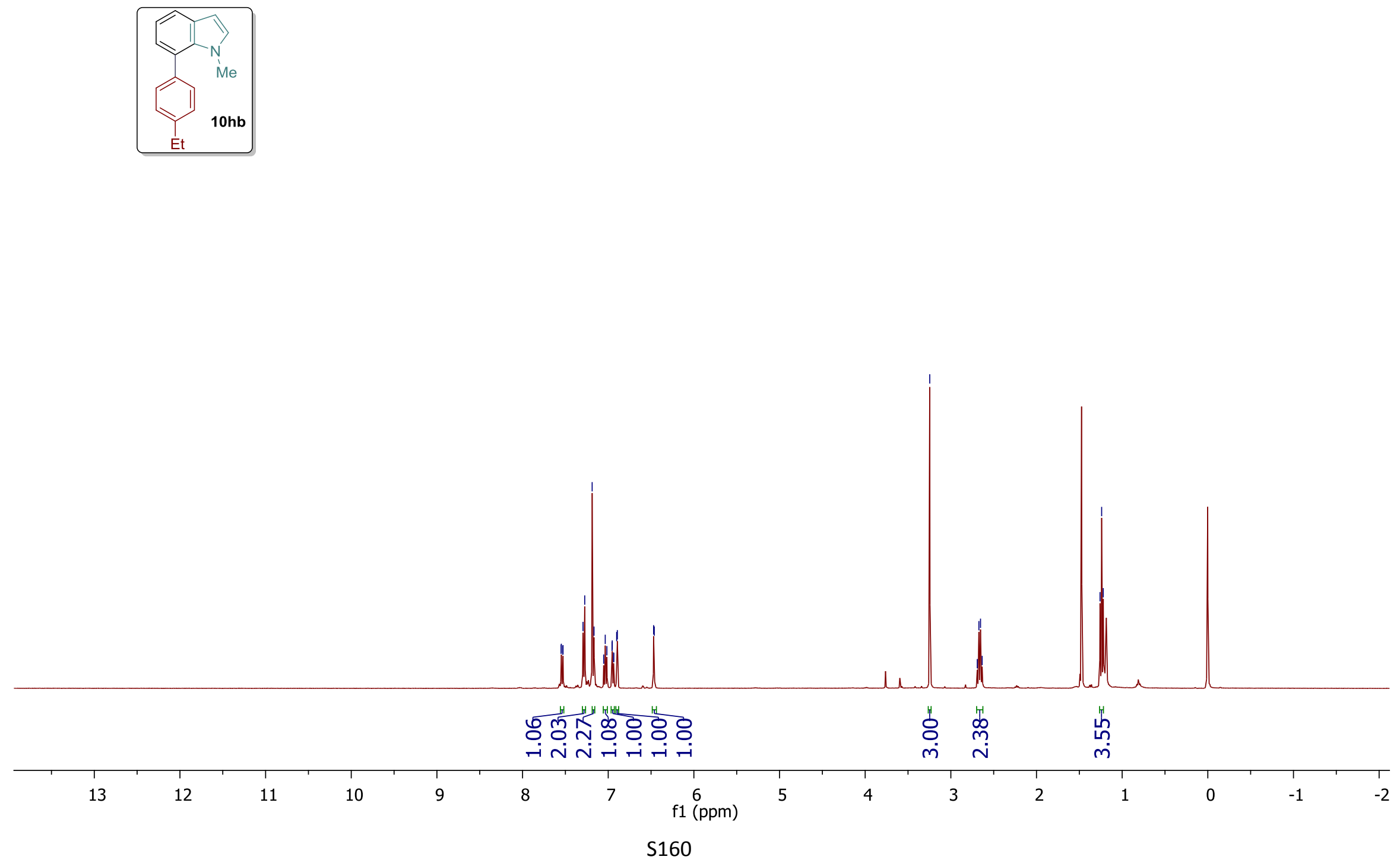

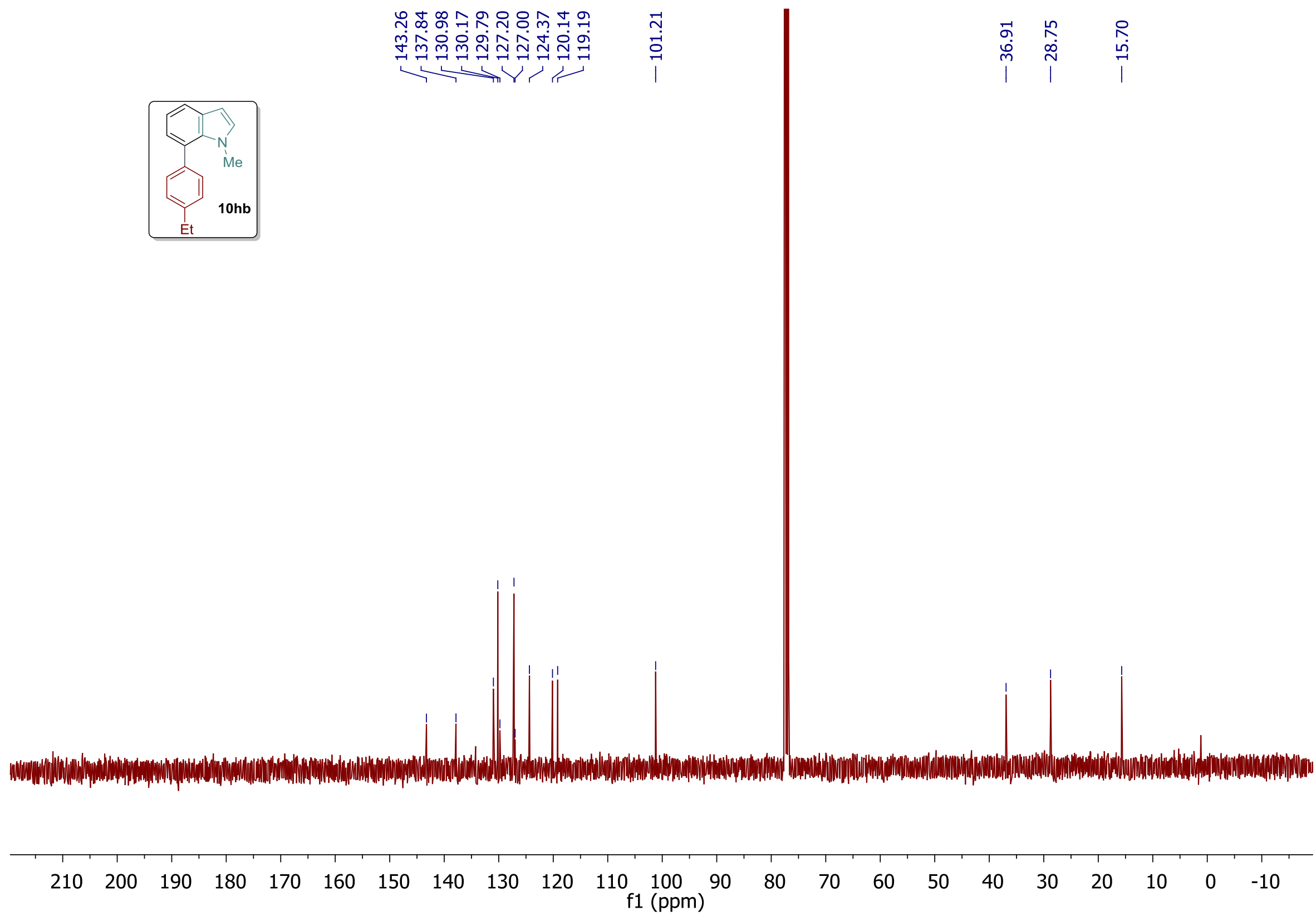
S161 

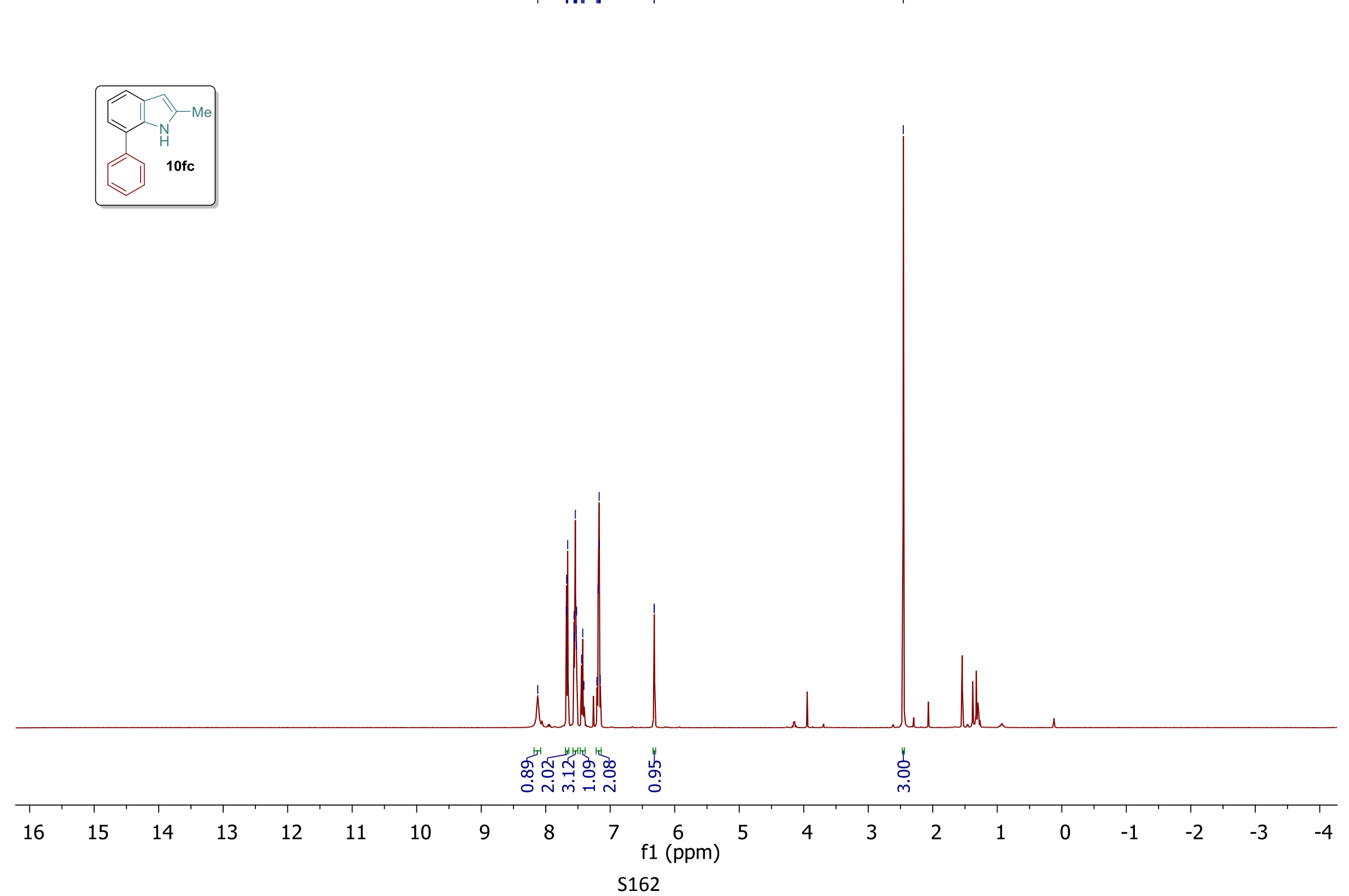

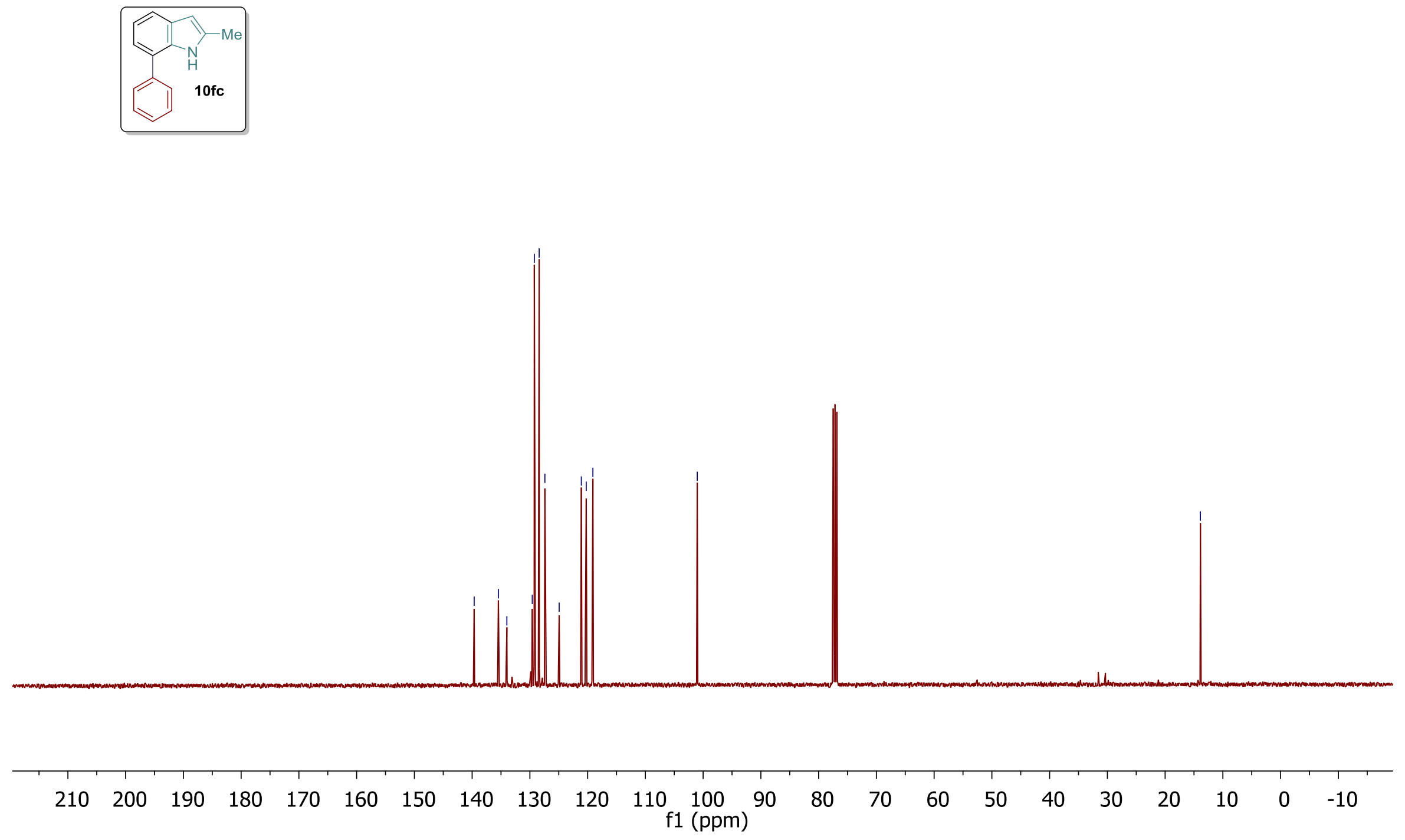
S163 


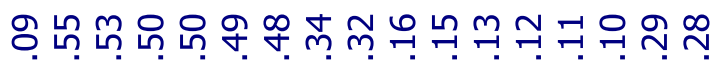

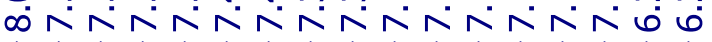
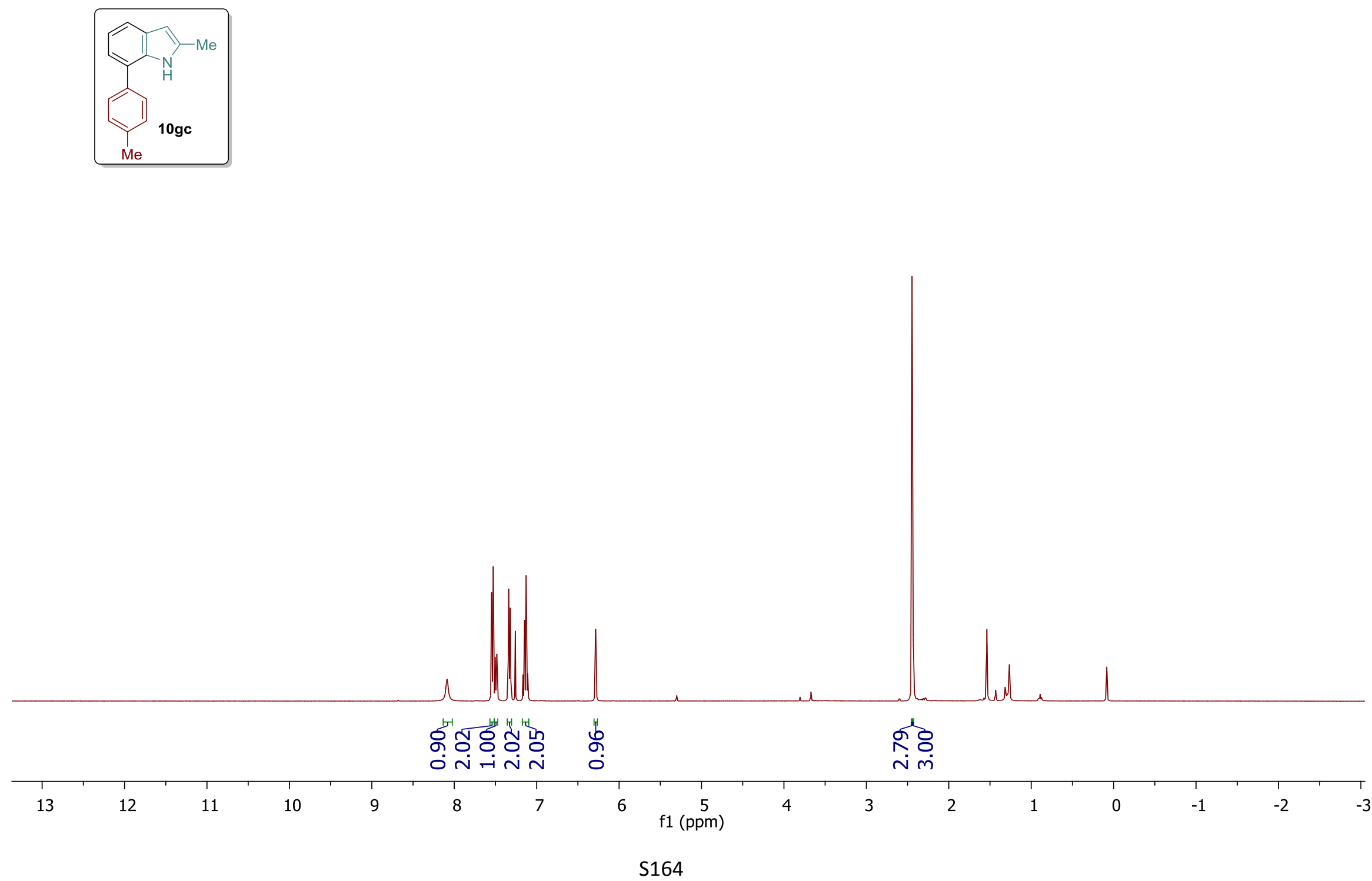


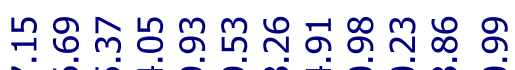

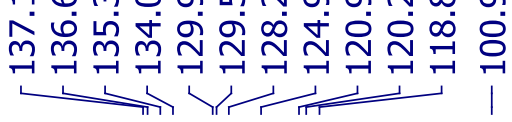

m 8

ㄱำ

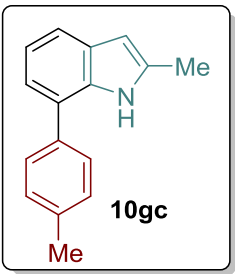




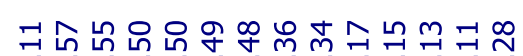

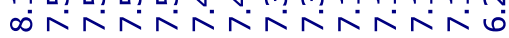

mananisiniso

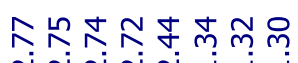

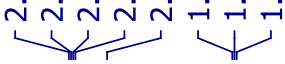
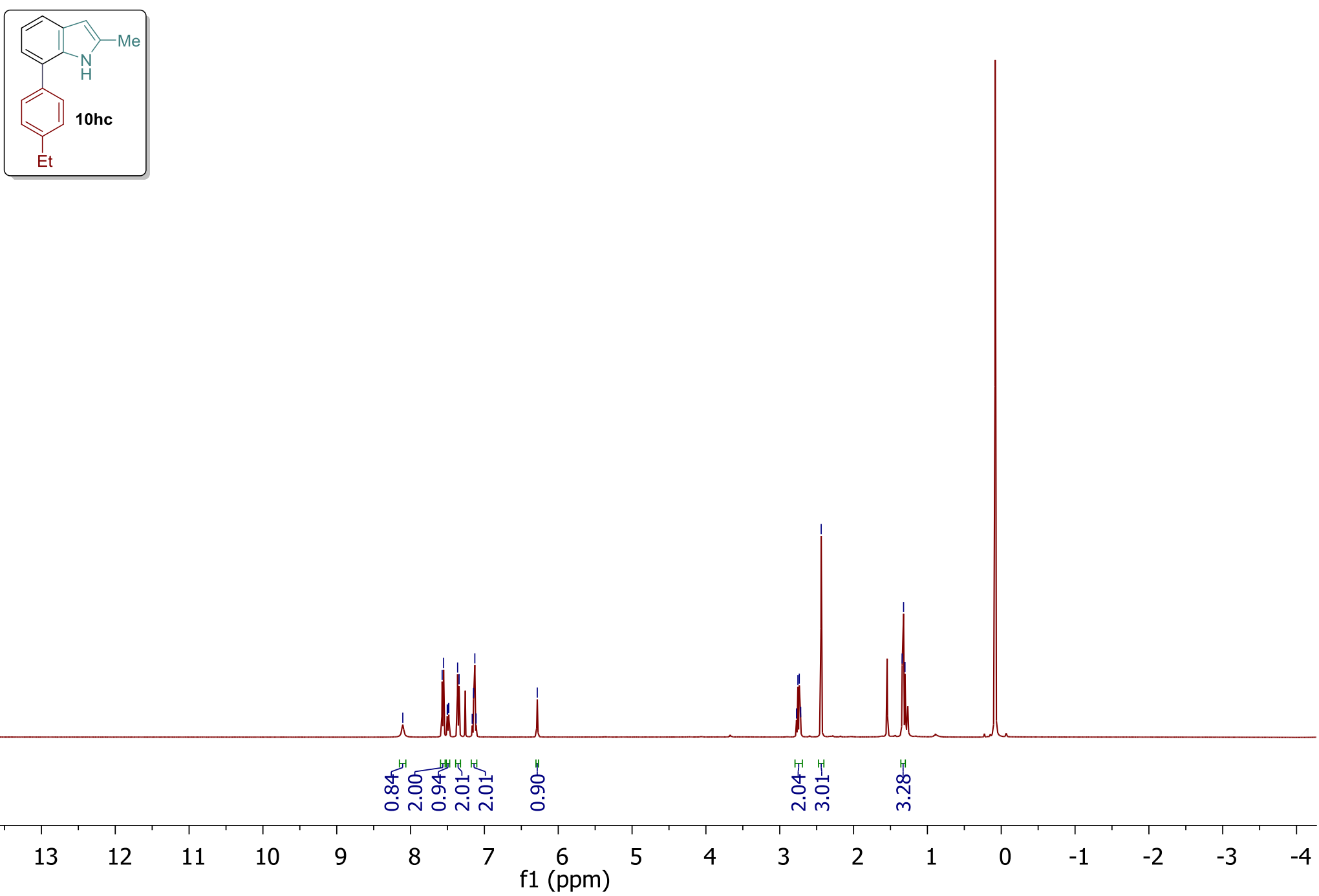


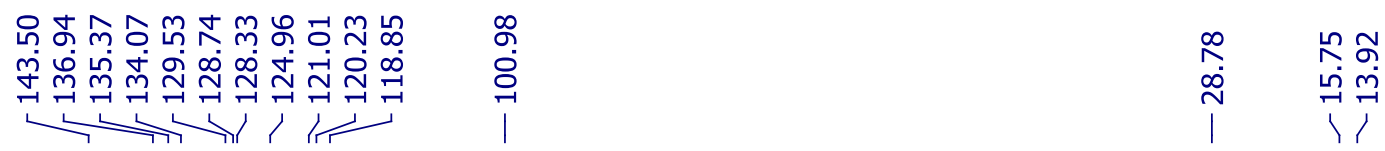
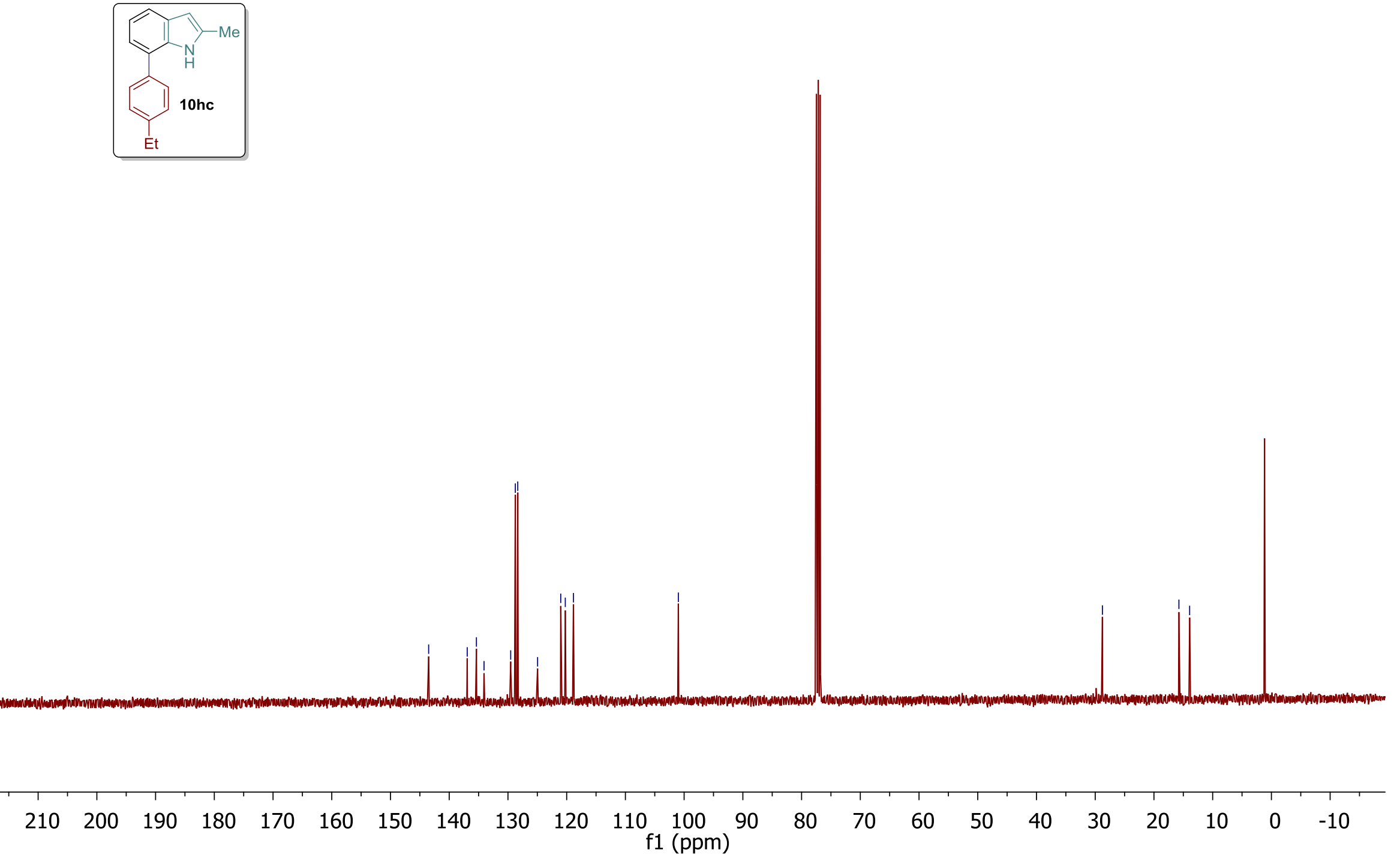


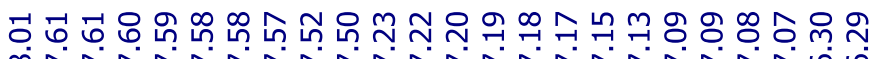

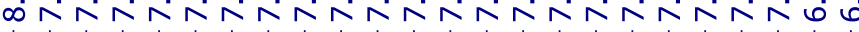
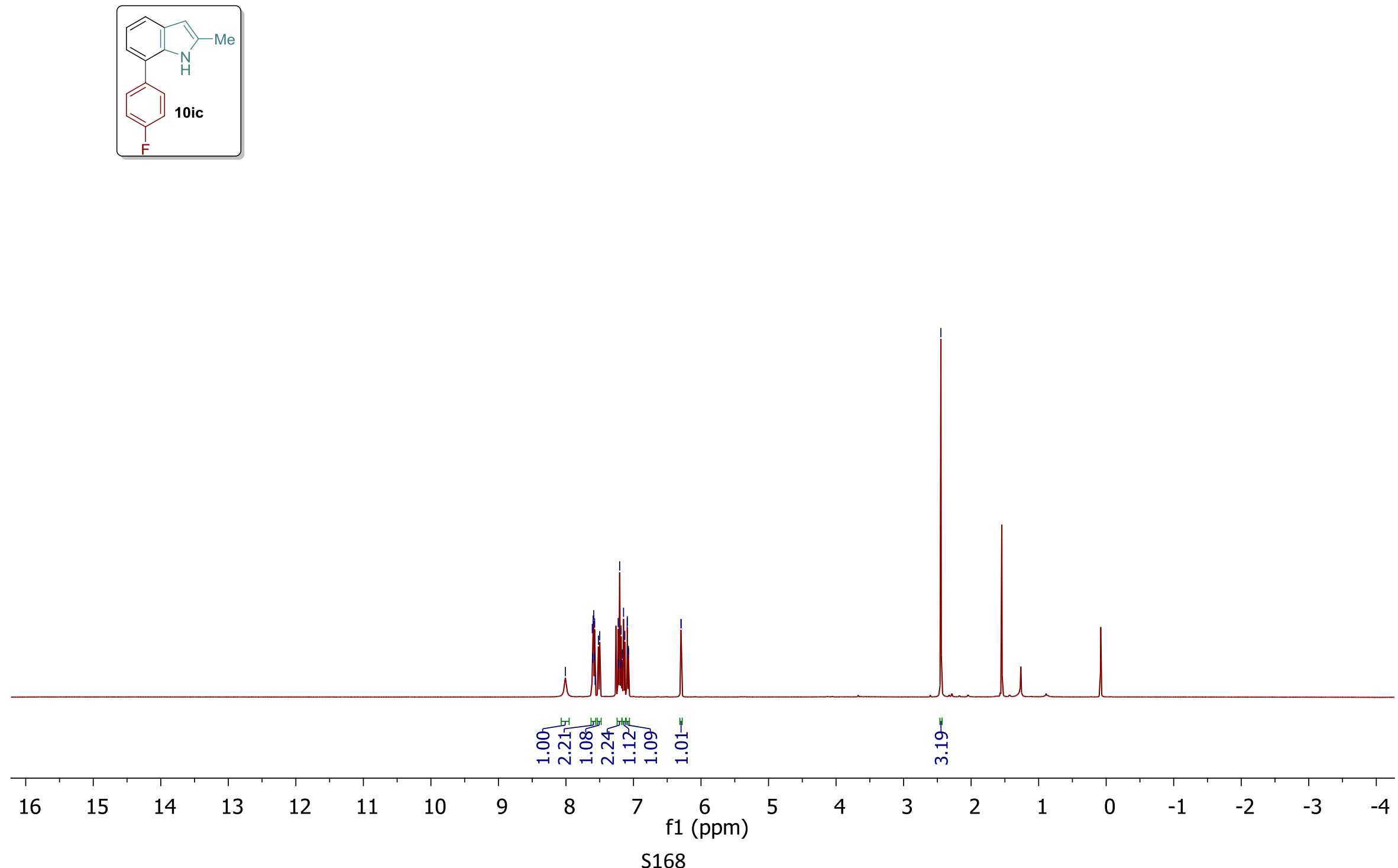

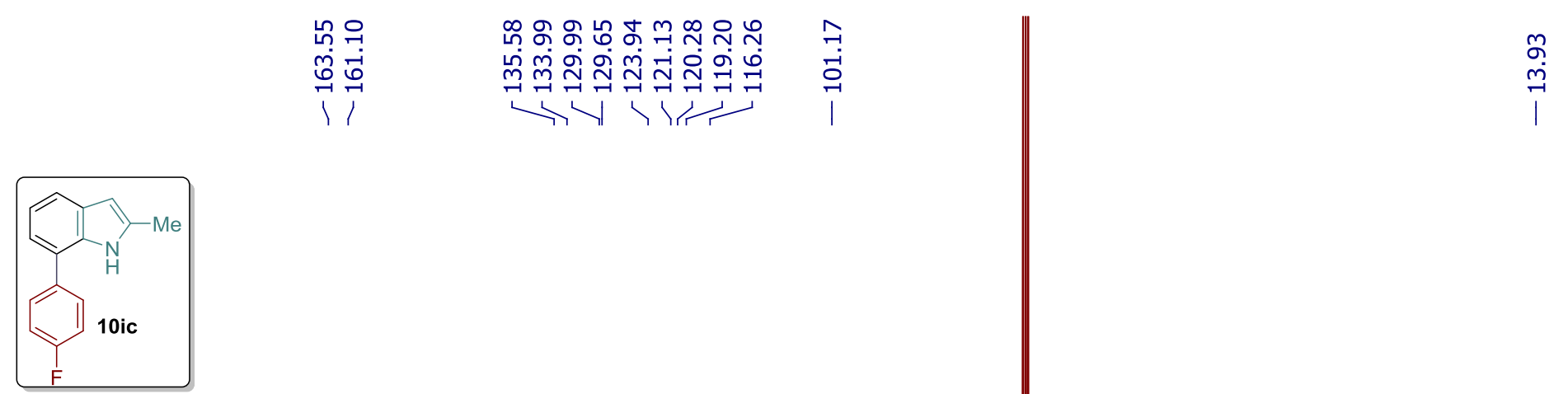

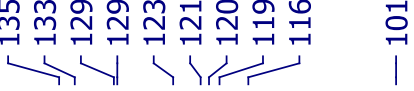

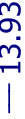
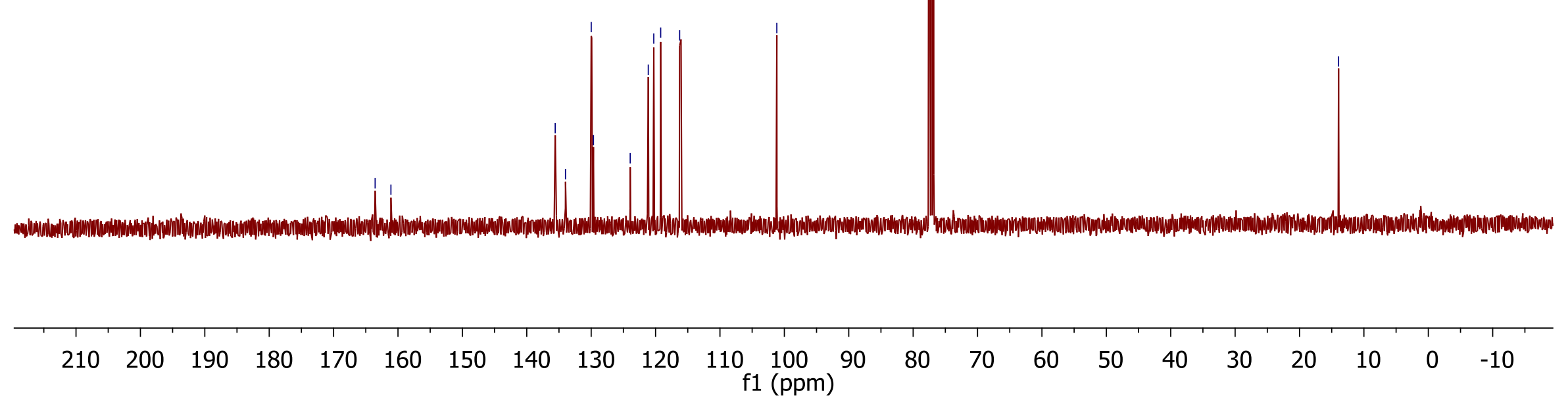
S169 


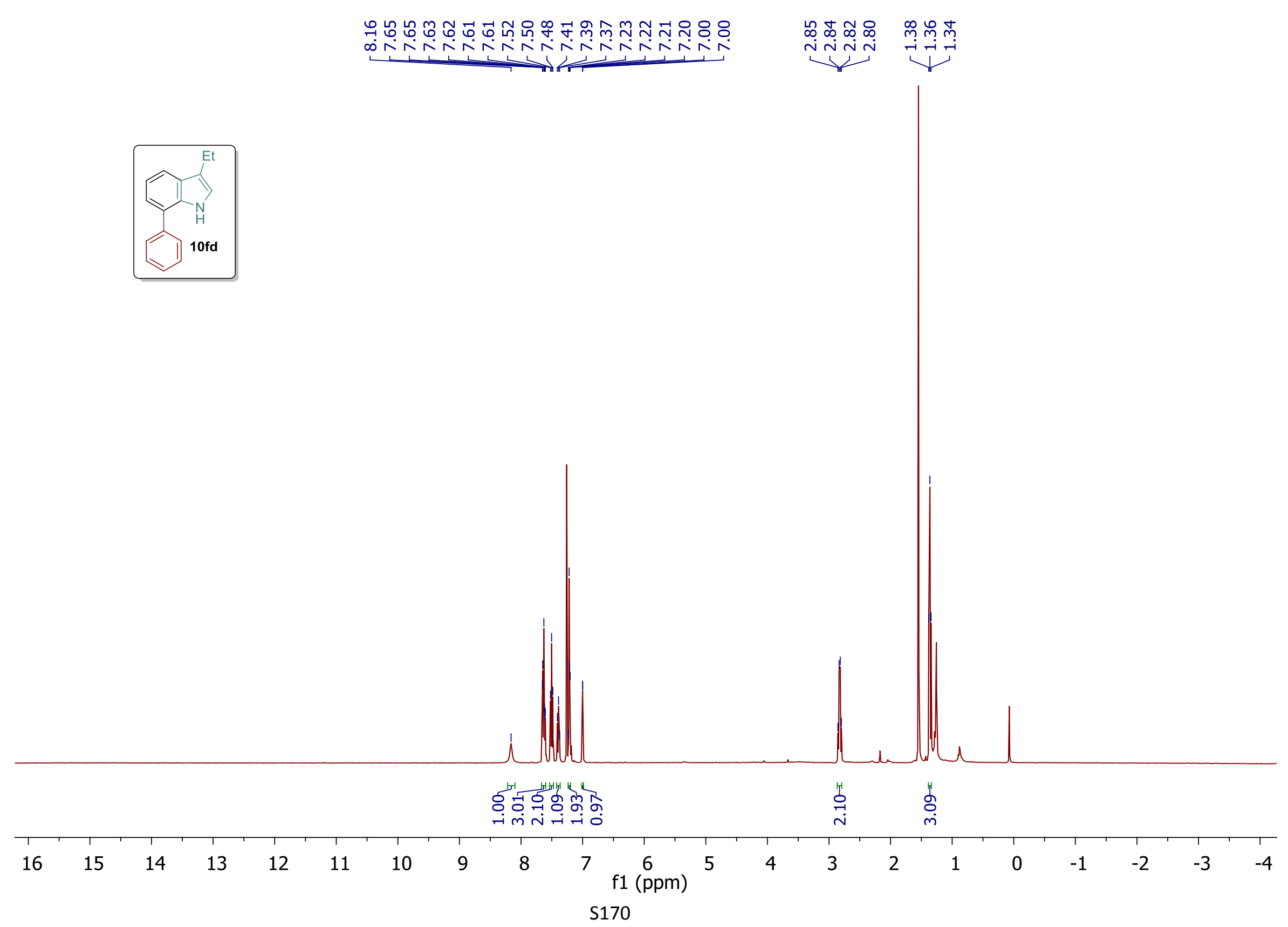



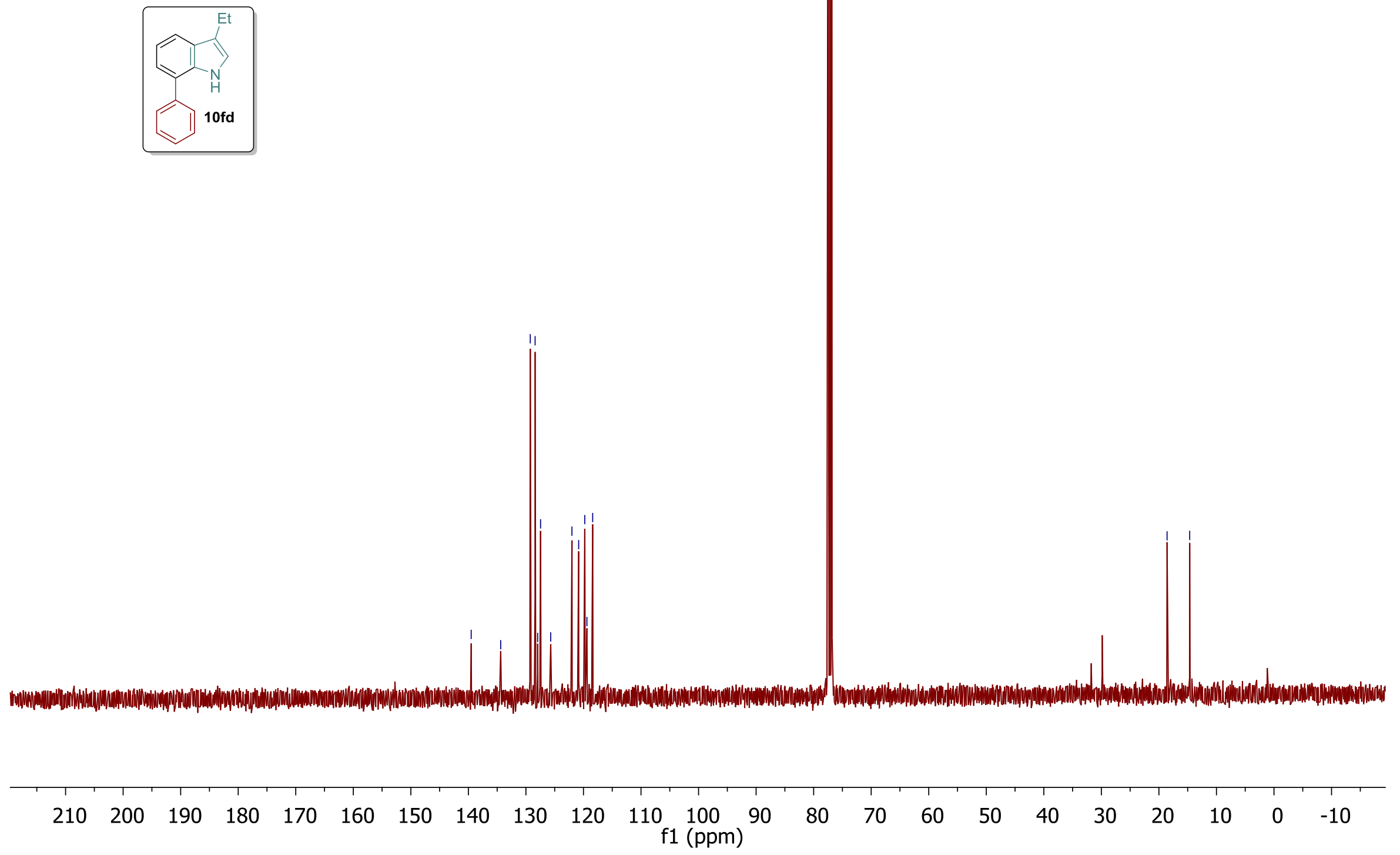
S171 


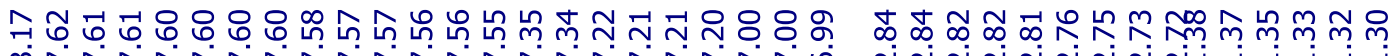

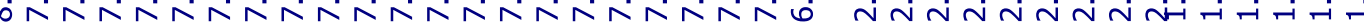
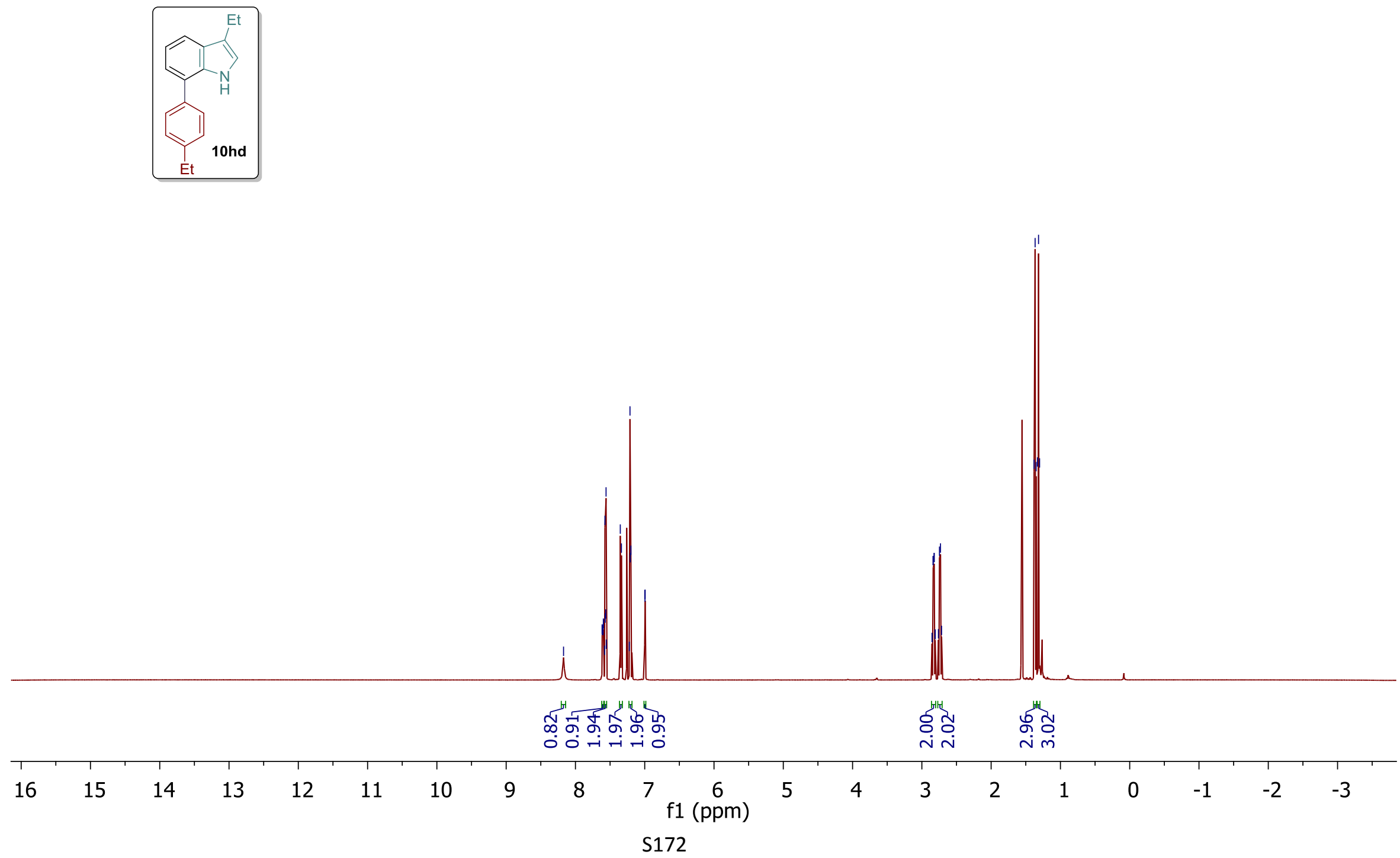
นกำๆ

守

人 ㅇํำํํำ

望

舟

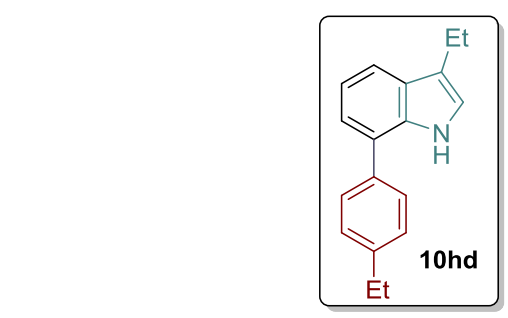

$1<1$

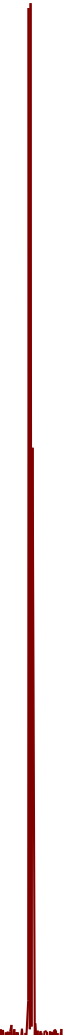

\begin{tabular}{|c|c|c|c|c|c|c|c|c|c|c|c|c|c|c|c|c|c|c|c|}
\hline 1 & 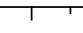 & 1 & $T$ & $\begin{array}{lll} & 1\end{array}$ & $T$ & $T$ & $T$ & $T$ & 1 & $T$ & 1 & $T$ & $T$ & $T$ & $T$ & $T$ & $T$ & 1 & $T$ \\
\hline 250 & 230 & 210 & 190 & 170 & 150 & 130 & $\begin{array}{r}110 \\
\mathrm{f} 1\end{array}$ & & 80 & 70 & 60 & 50 & 40 & 30 & 20 & 10 & 0 & -20 & -40 \\
\hline
\end{tabular}




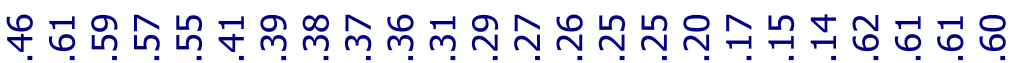

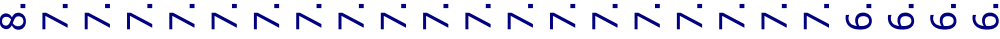
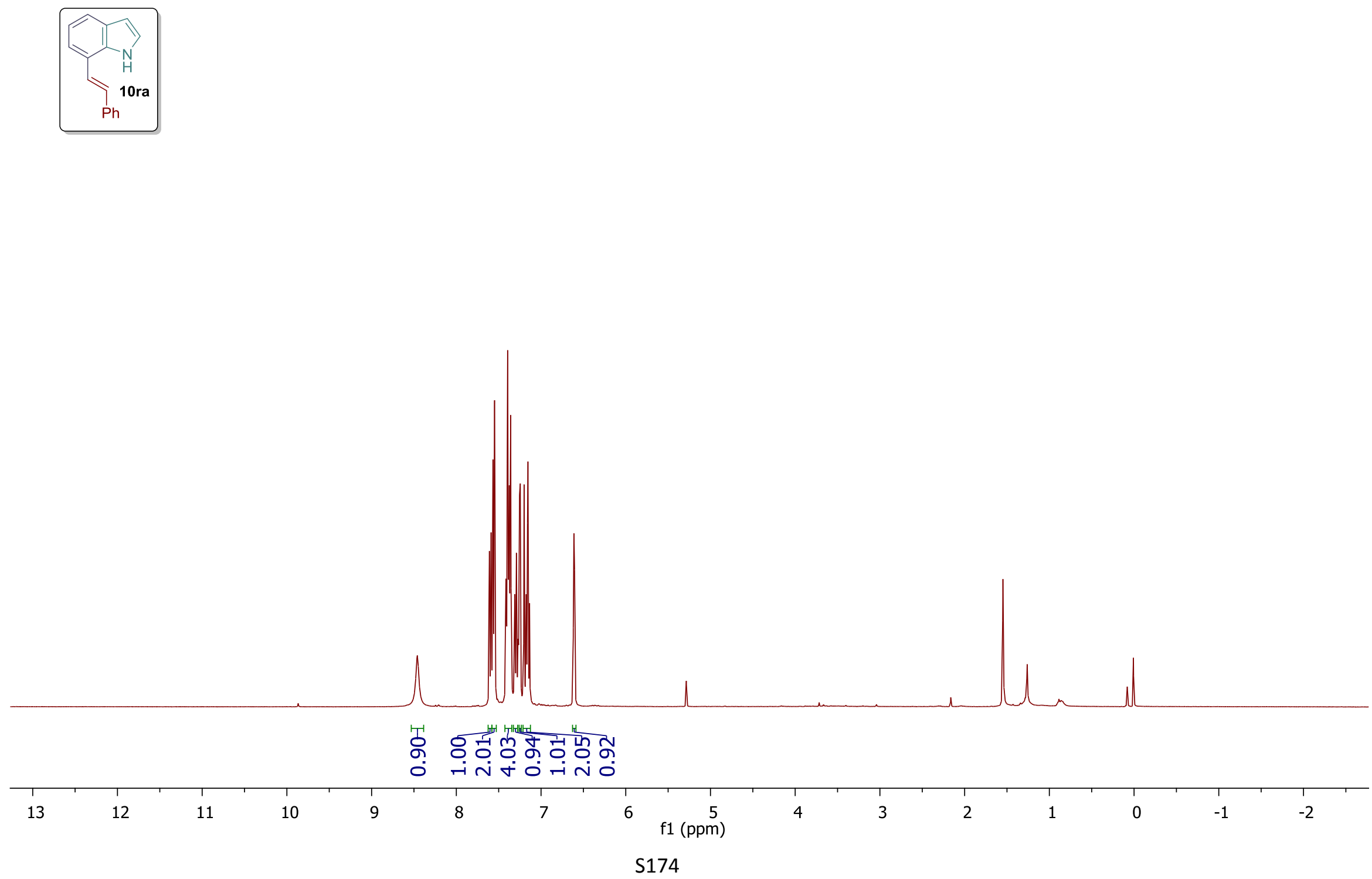
ชิ

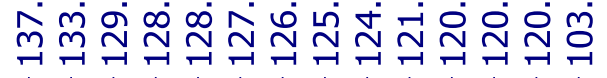

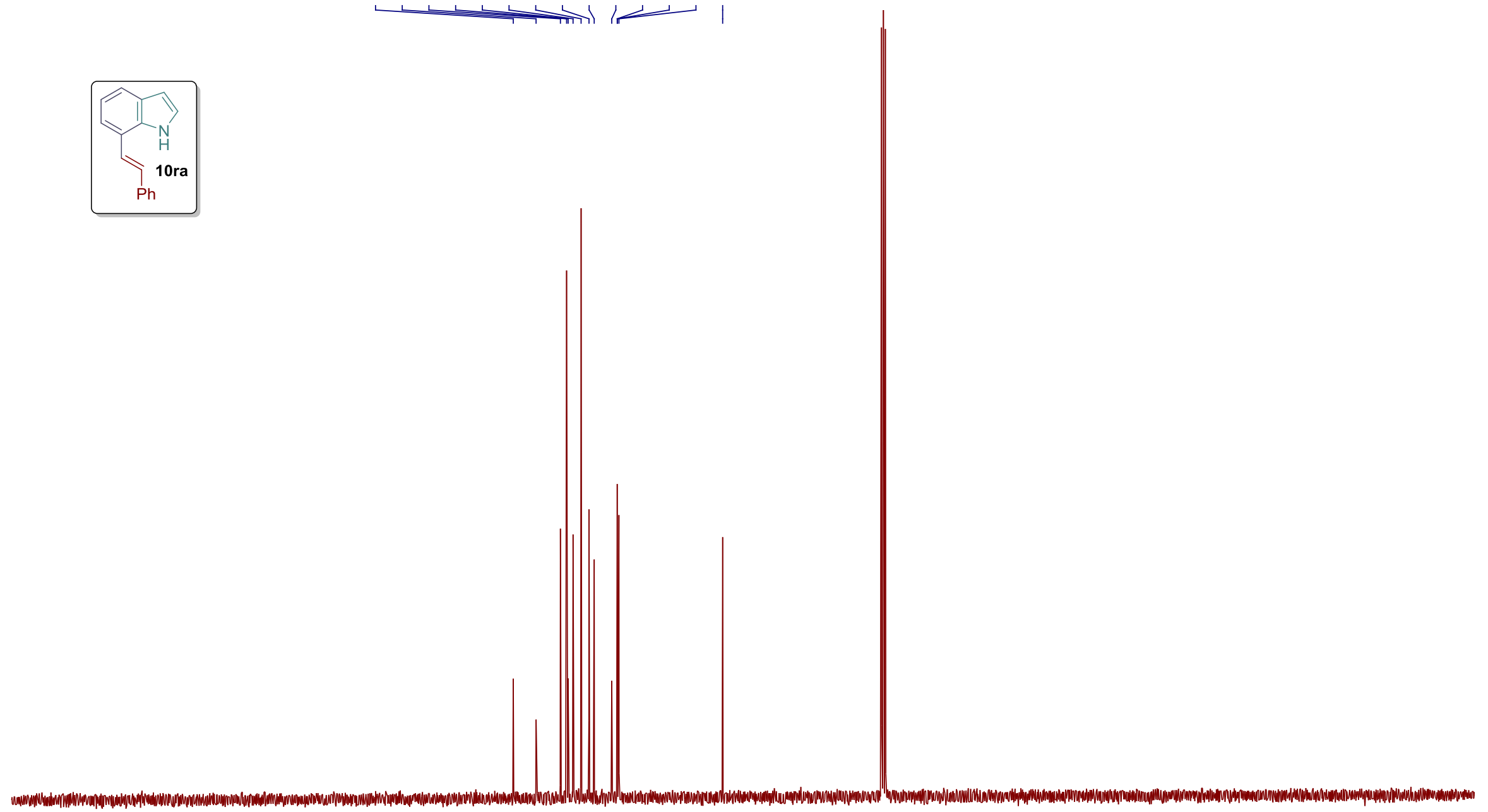

$\begin{array}{lllllllllllllllllllllllll}210 & 200 & 190 & 180 & 170 & 160 & 150 & 140 & 130 & 120 & 110 & \begin{array}{c}100 \\ \mathrm{f} 1(\mathrm{ppm})\end{array} & 90 & 80 & 70 & 60 & 50 & 40 & 30 & 20 & 10 & 0 & -10 & \end{array}$ 


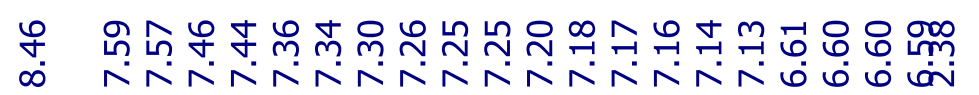

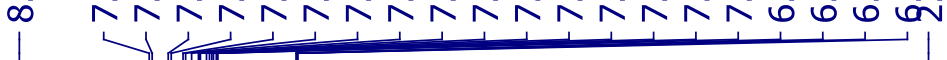
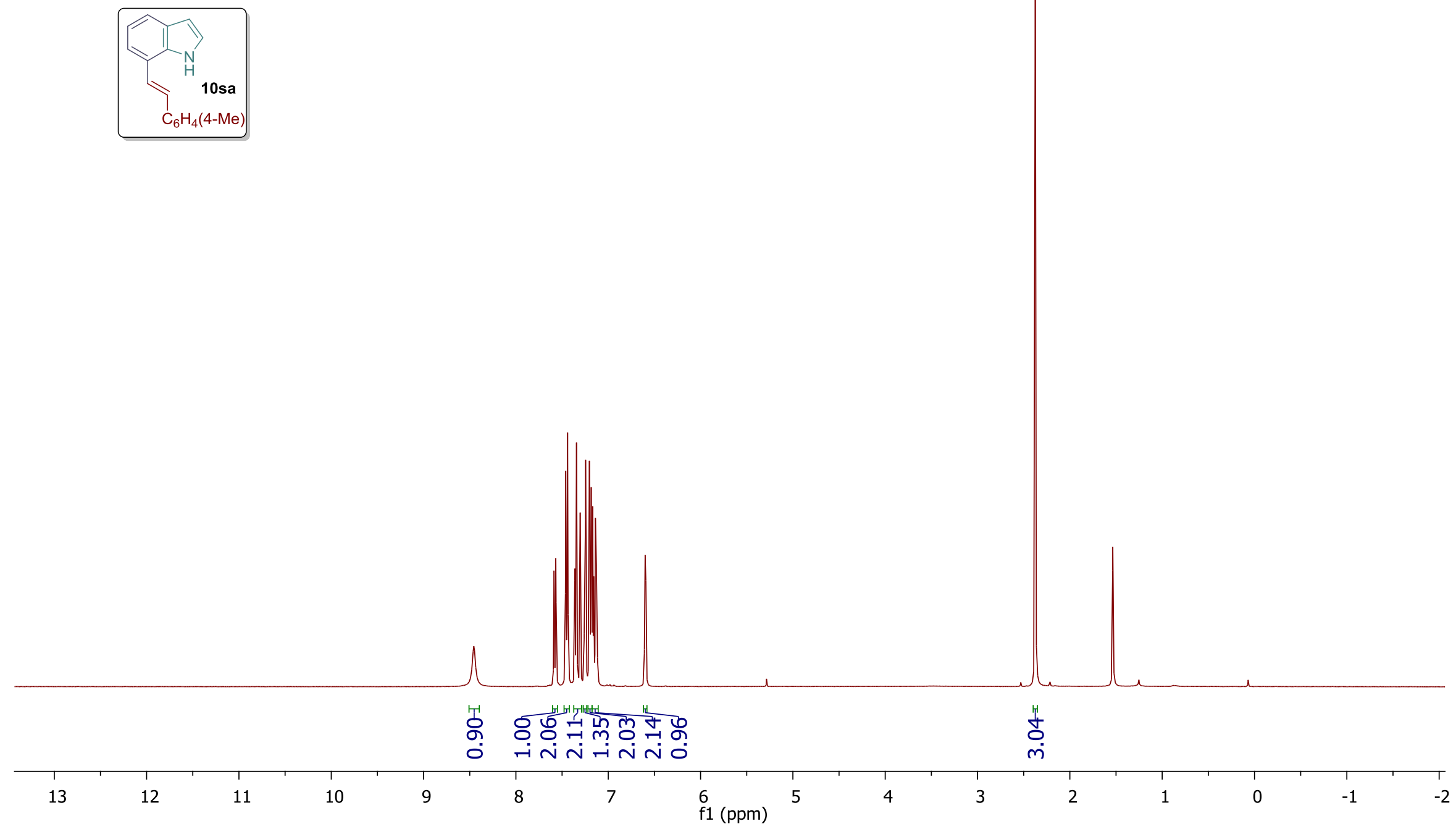
ळ

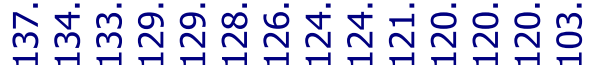
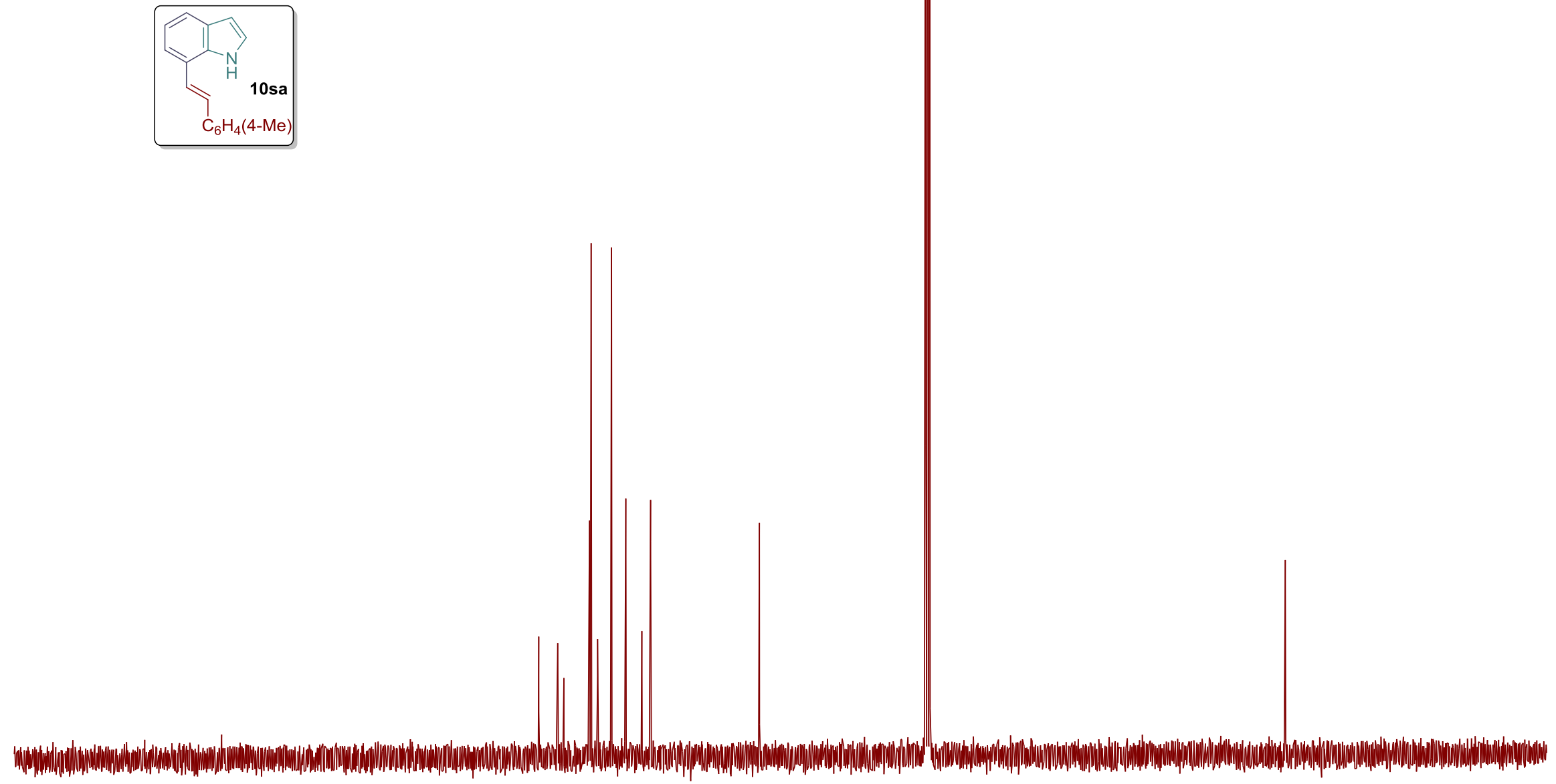


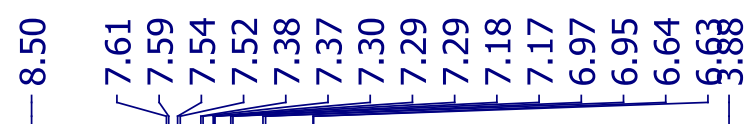

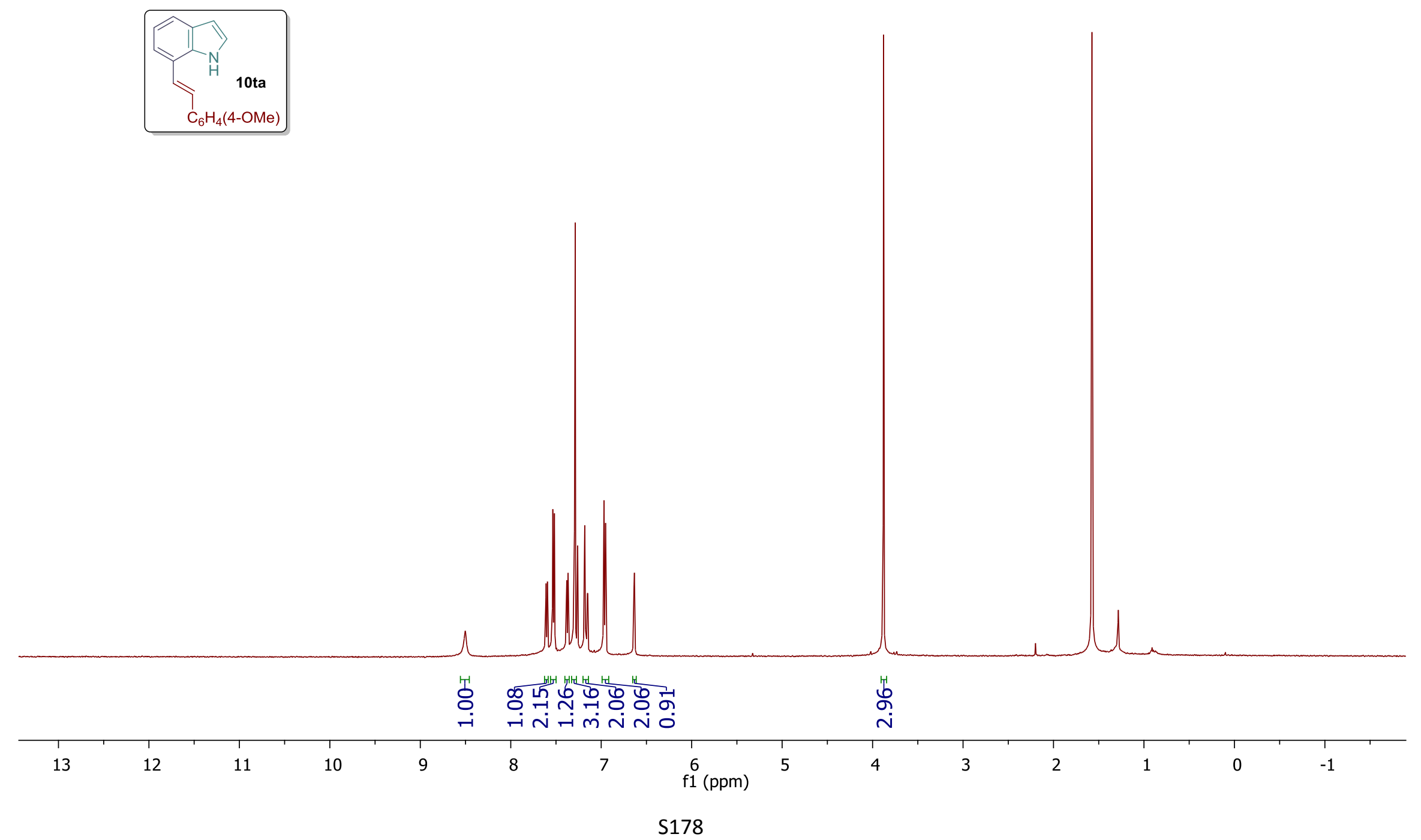




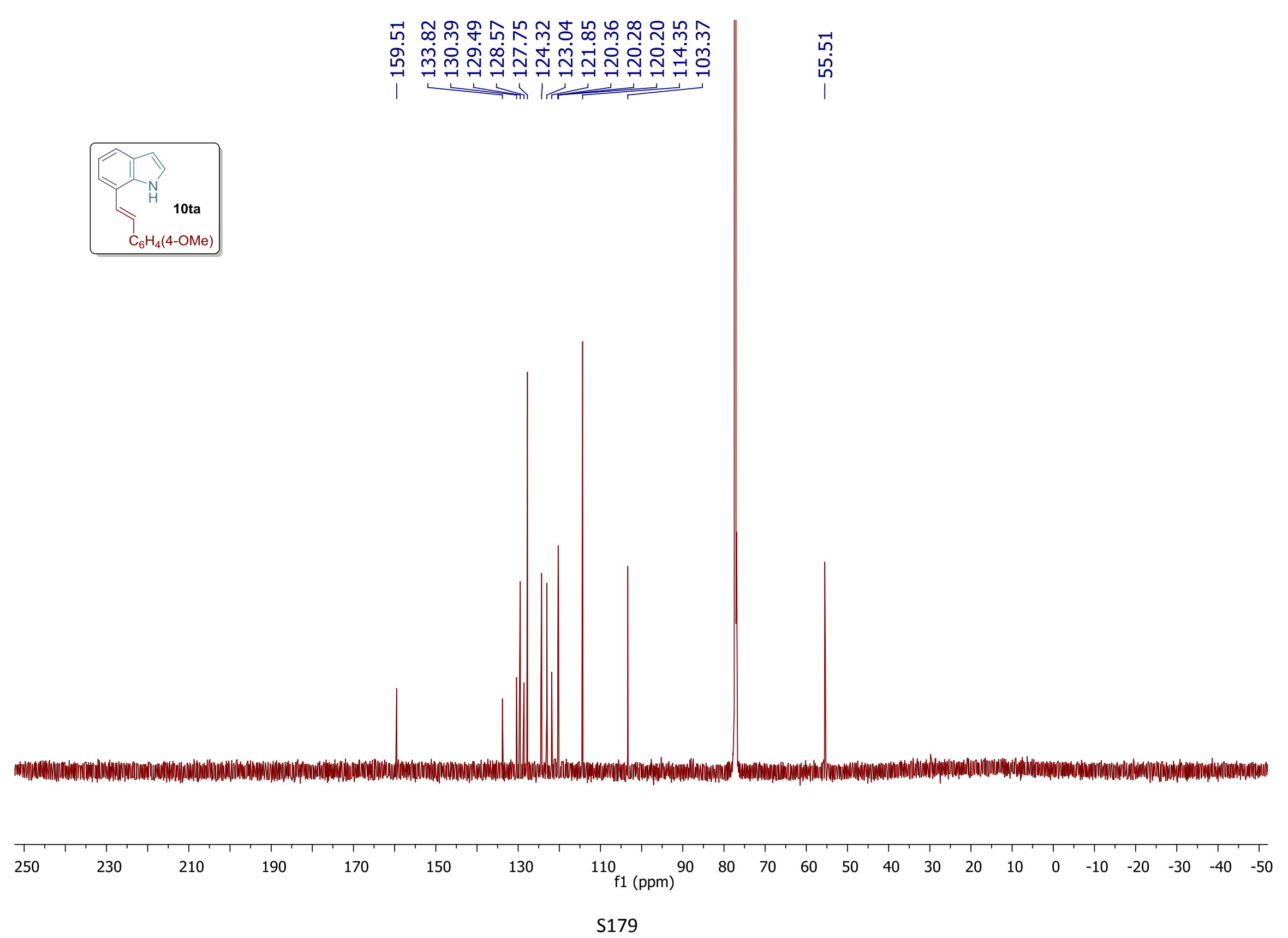




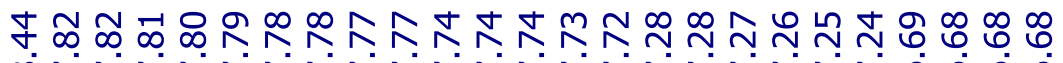

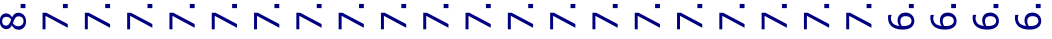
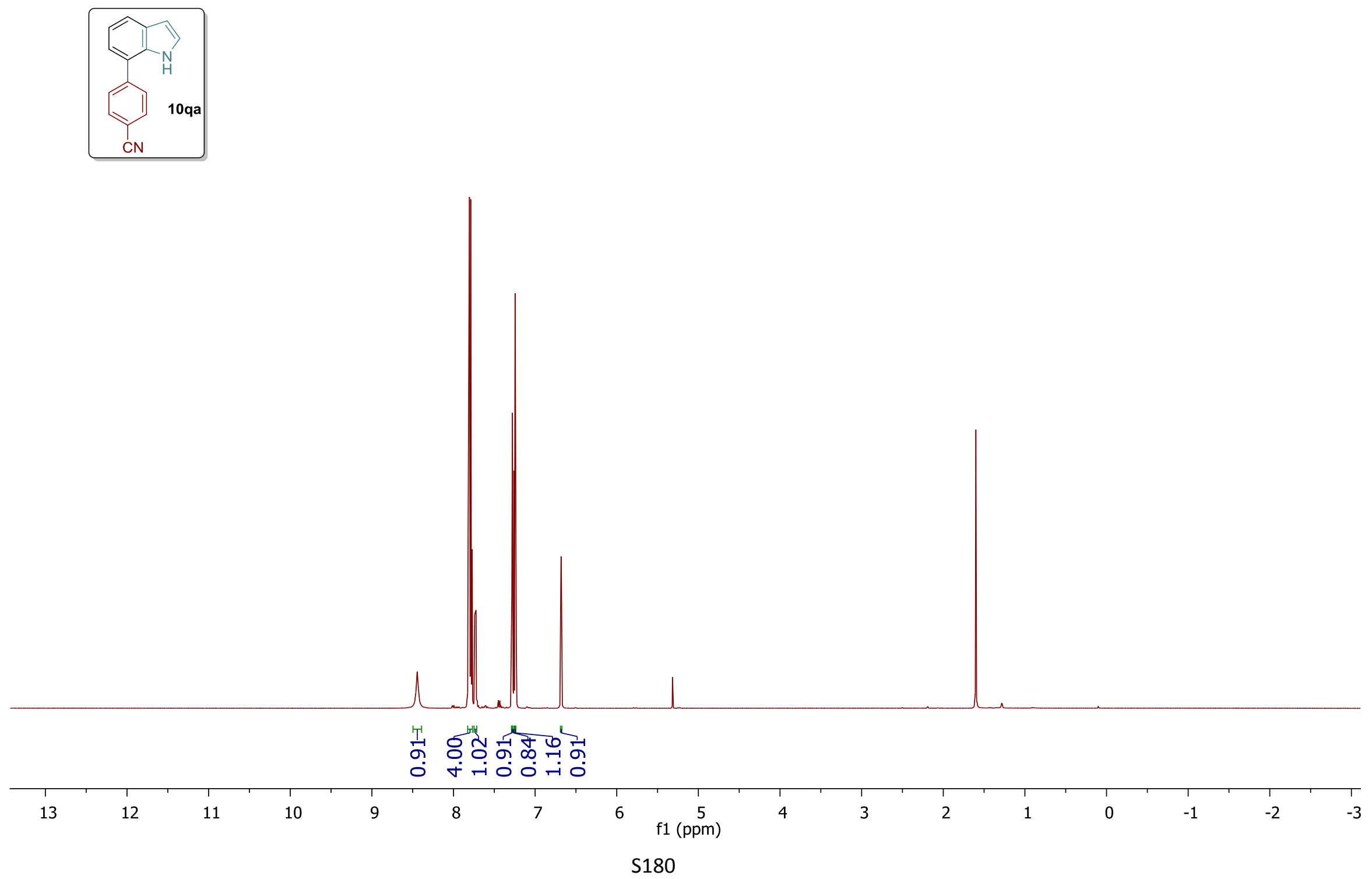


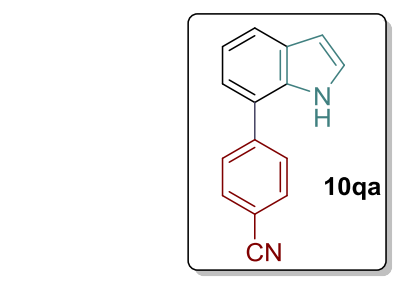

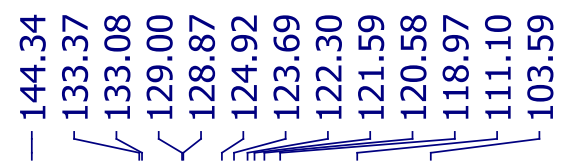
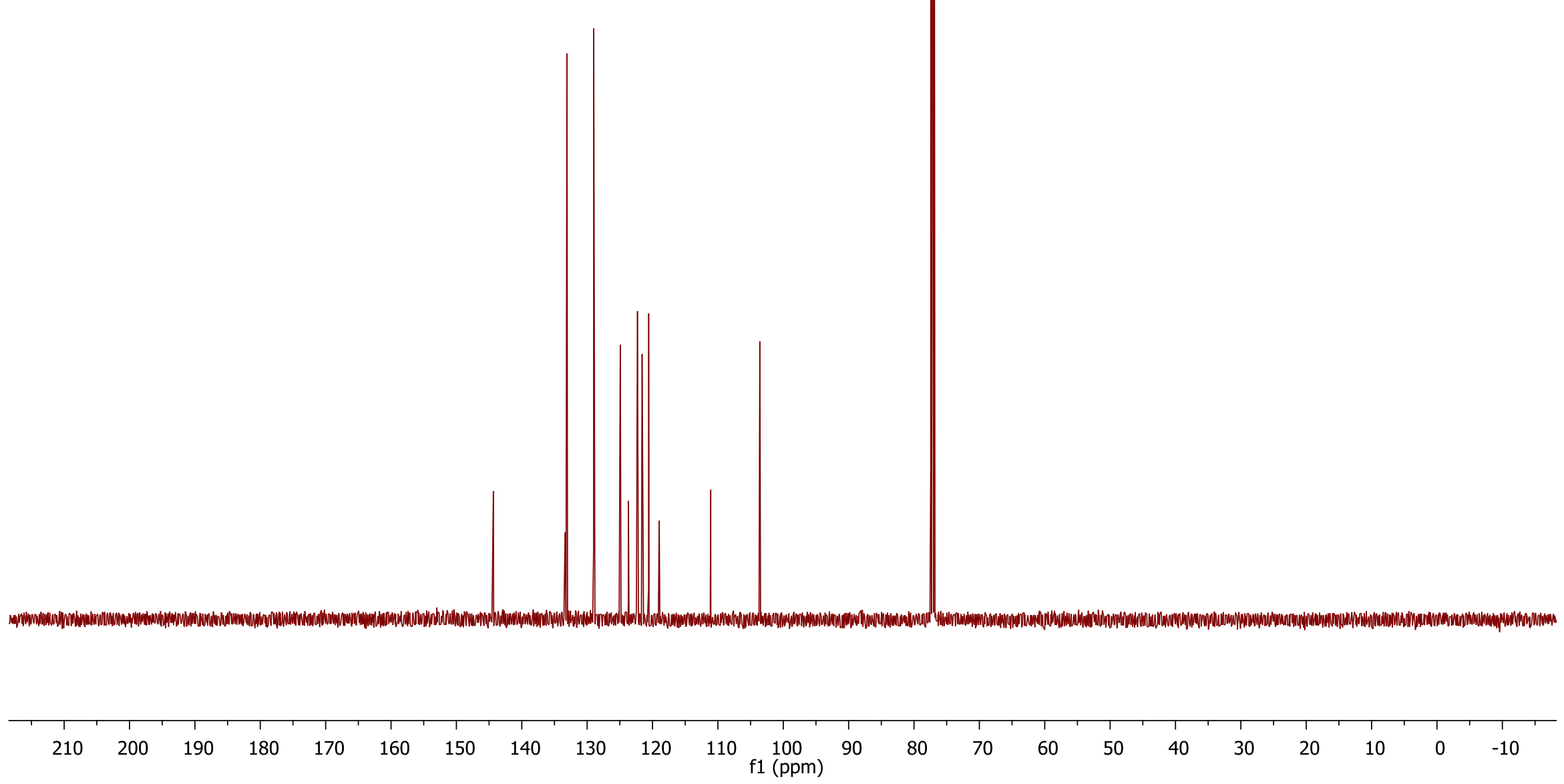

S181 


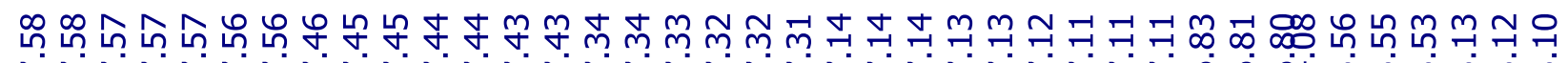

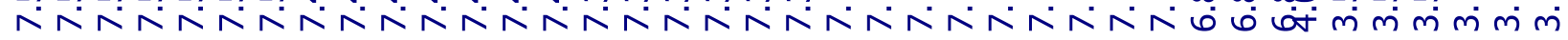
(1)
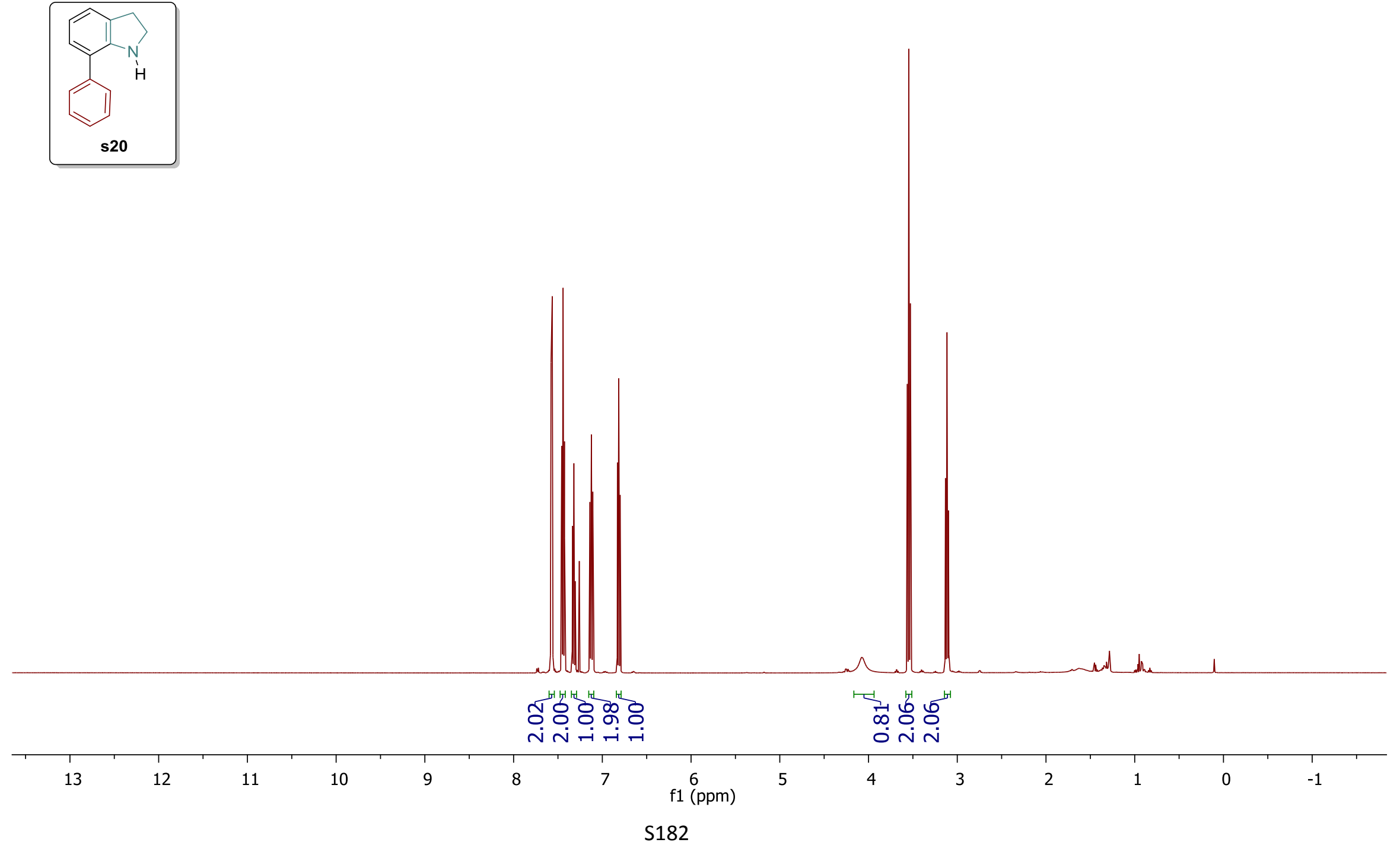


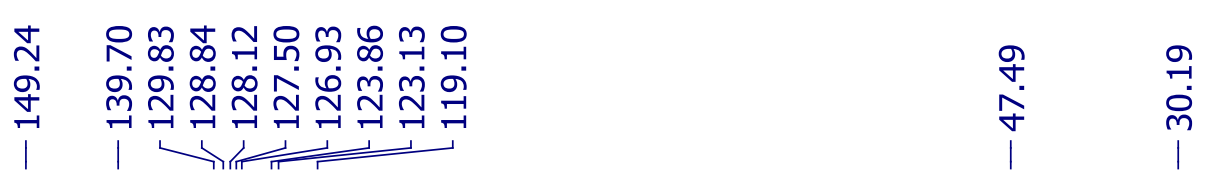
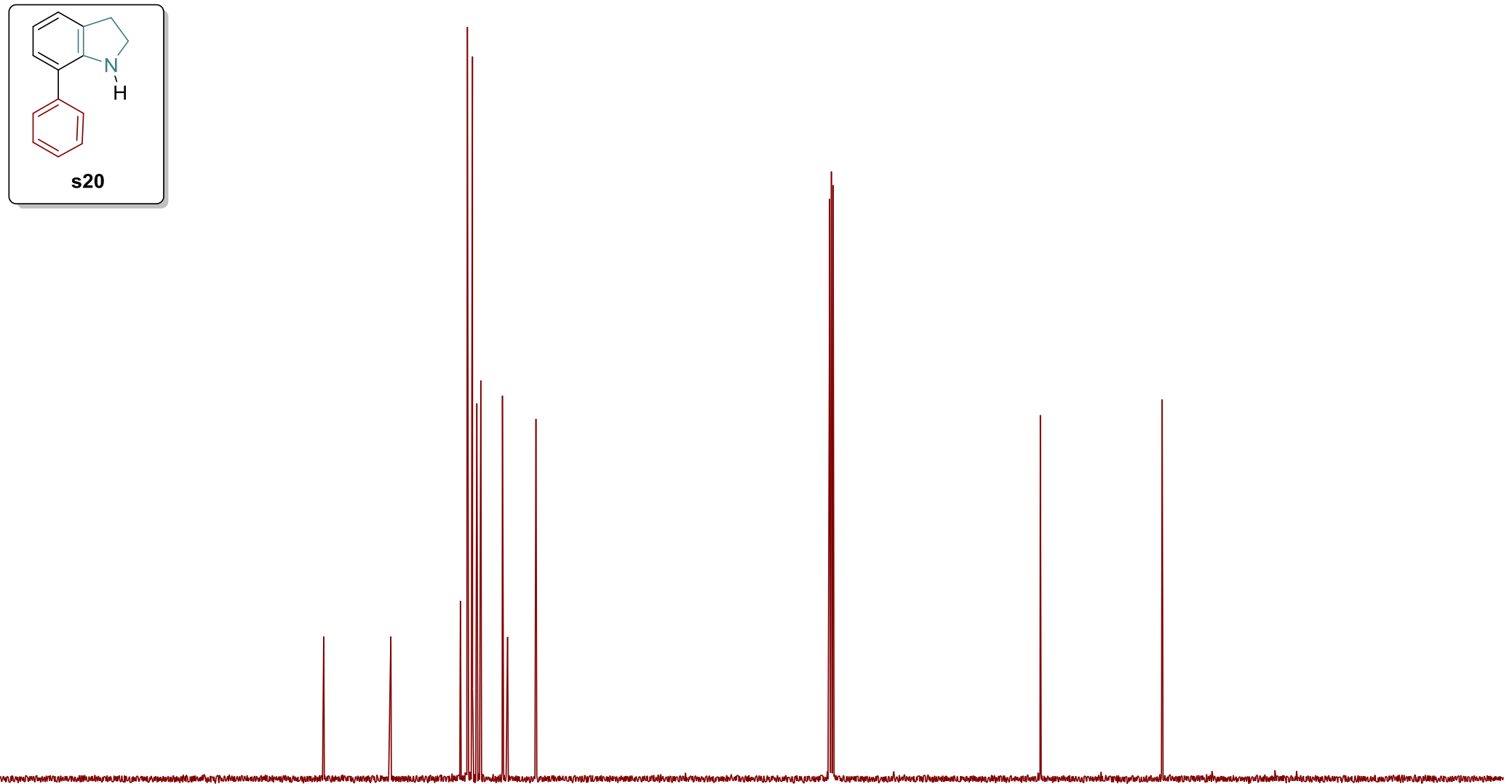

$210 \quad 200 \quad 190$

$180 \quad 170$

160
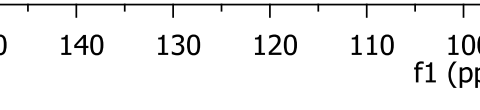


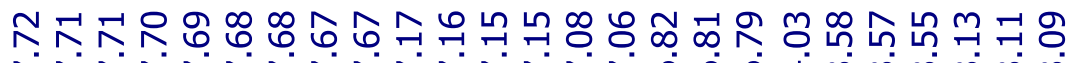

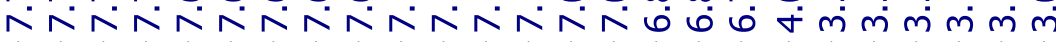
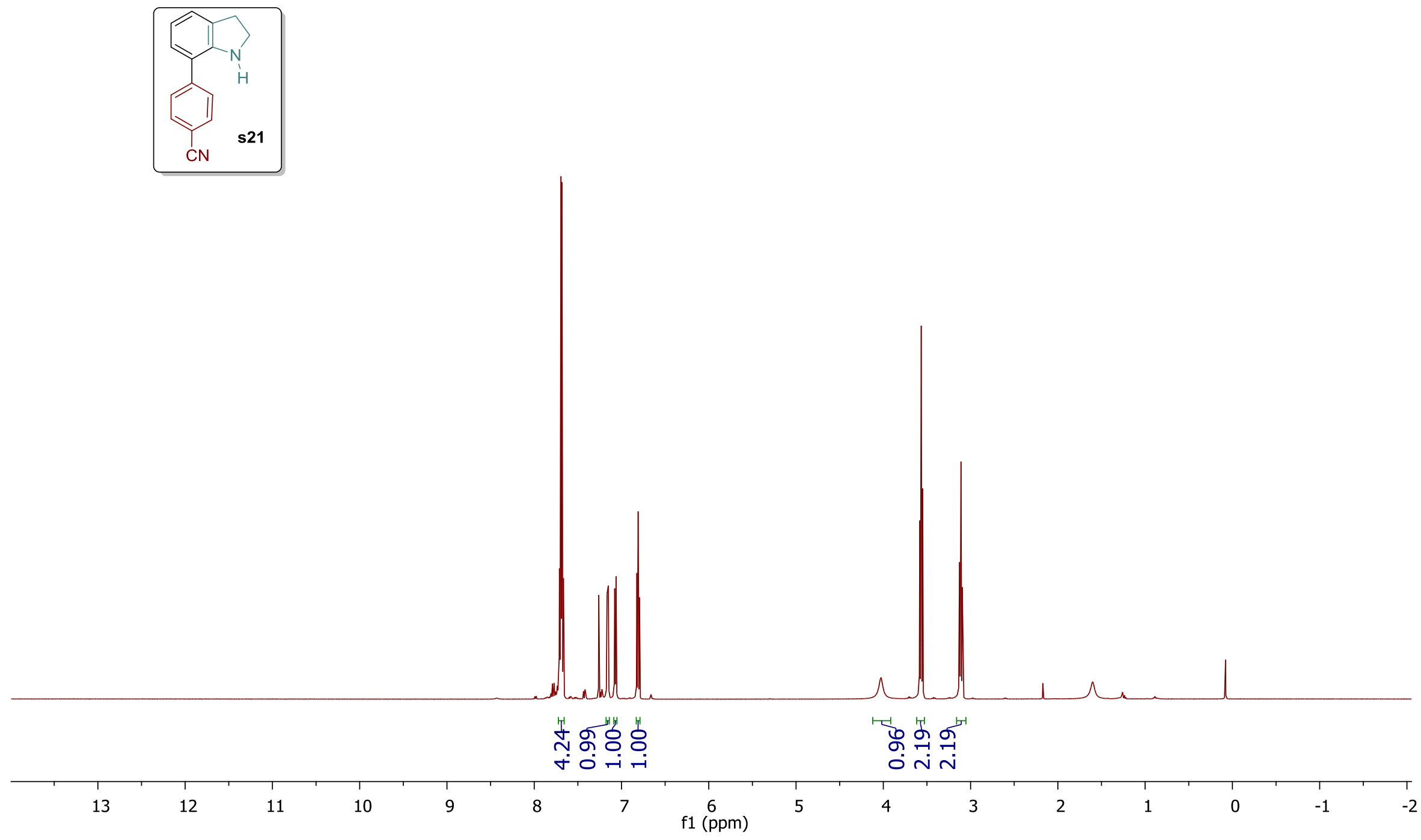


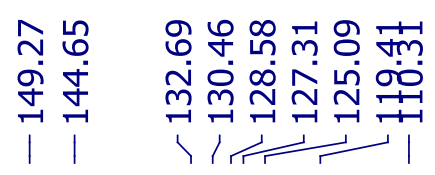

$\begin{array}{ll}\text { ₹ } & \text { ने } \\ \text { 1 } & \text { กे }\end{array}$
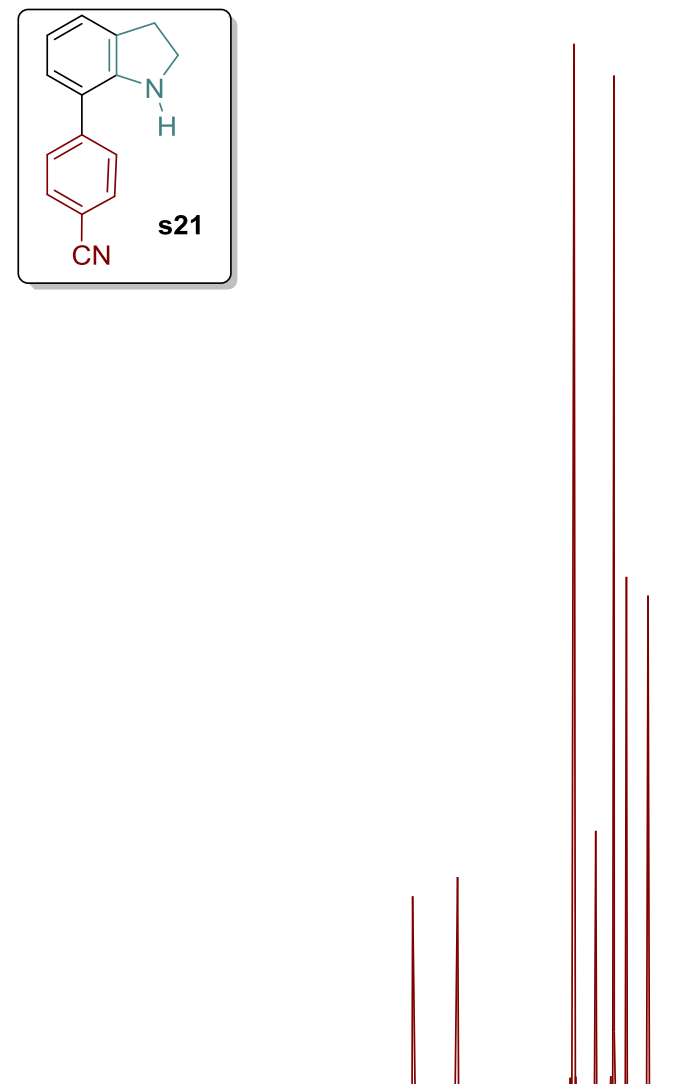

$210 \quad 200 \quad 190$

$180 \quad 170$

160

150

$140 \quad 130$

120

$110 \begin{gathered}100 \\ \mathrm{f} 1(\mathrm{ppm})\end{gathered}$

80

70

60

50

$40 \quad 30$

$20 \quad 10$ 


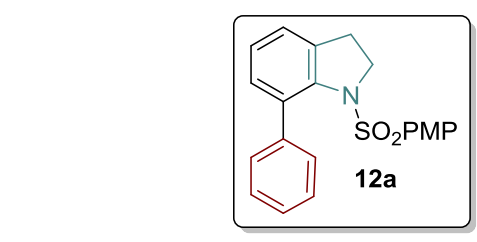
(1)

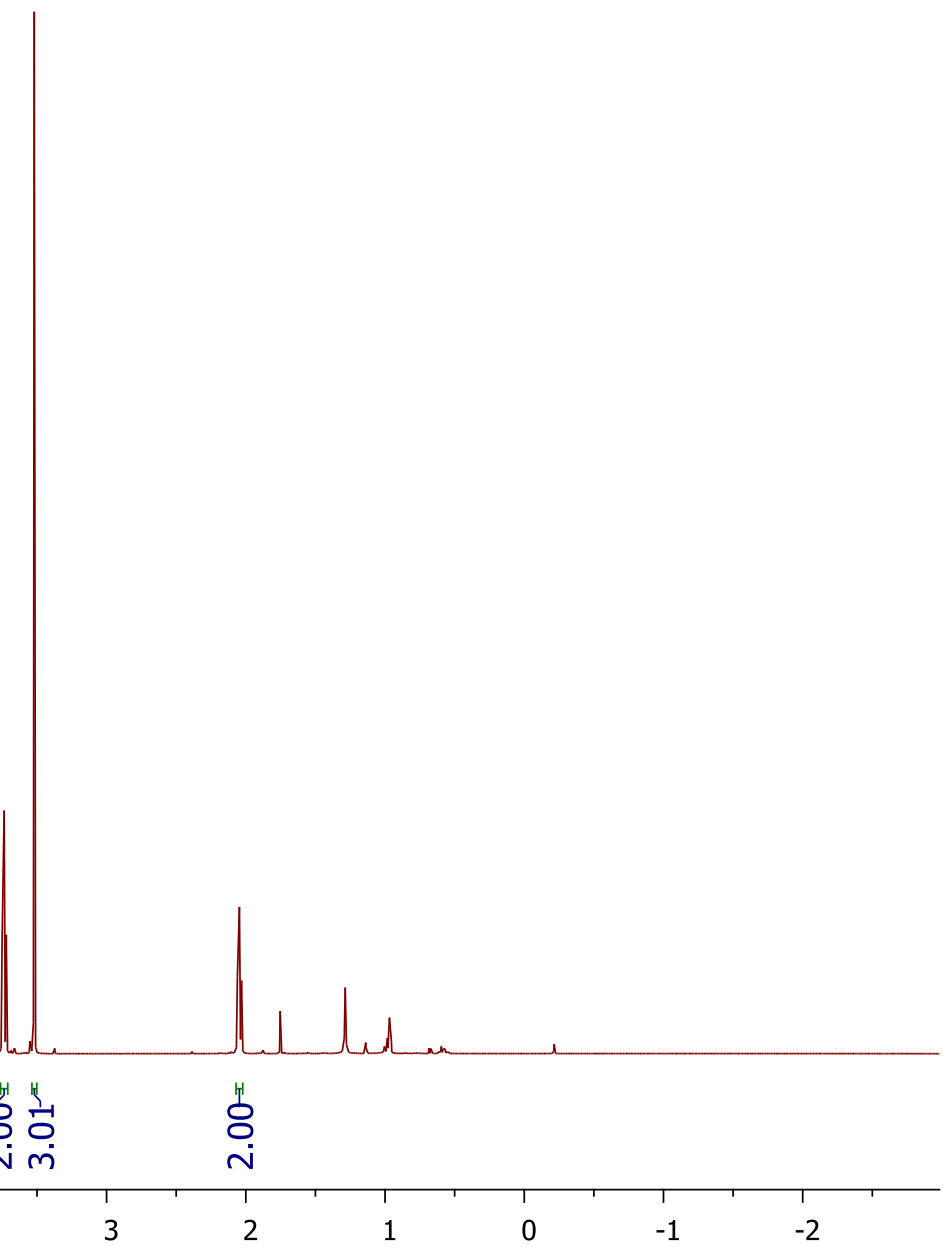




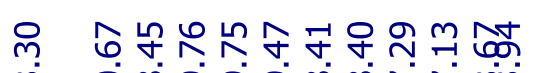

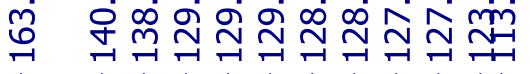

1 L L L L

$\widehat{6}$

นี่ กิ่

$\vec{m}$

|1

กิ
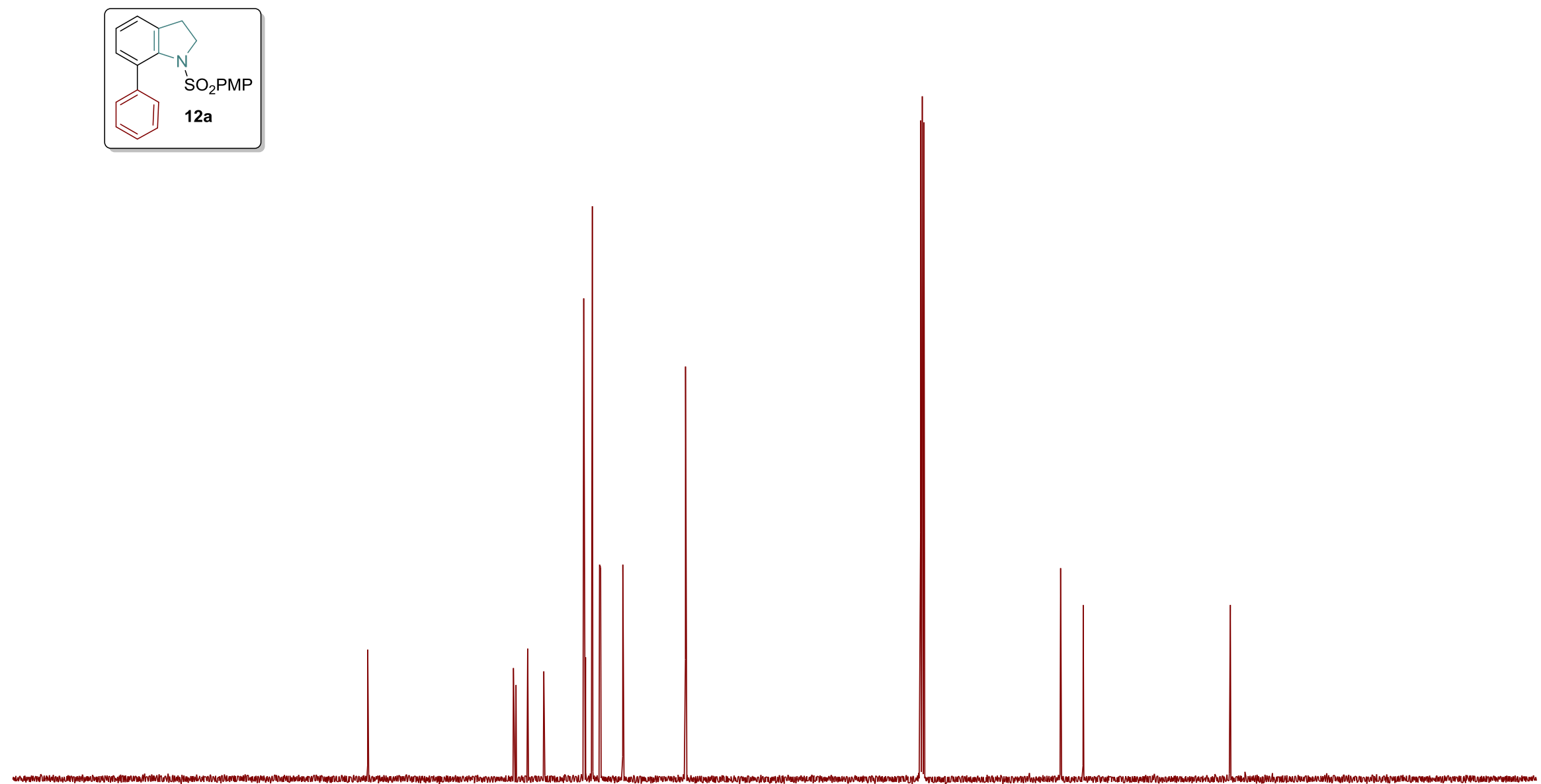


\begin{tabular}{|c|c|}
\hline 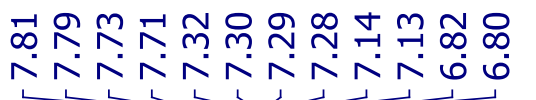 & 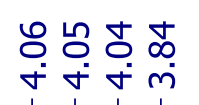 \\
\hline
\end{tabular}

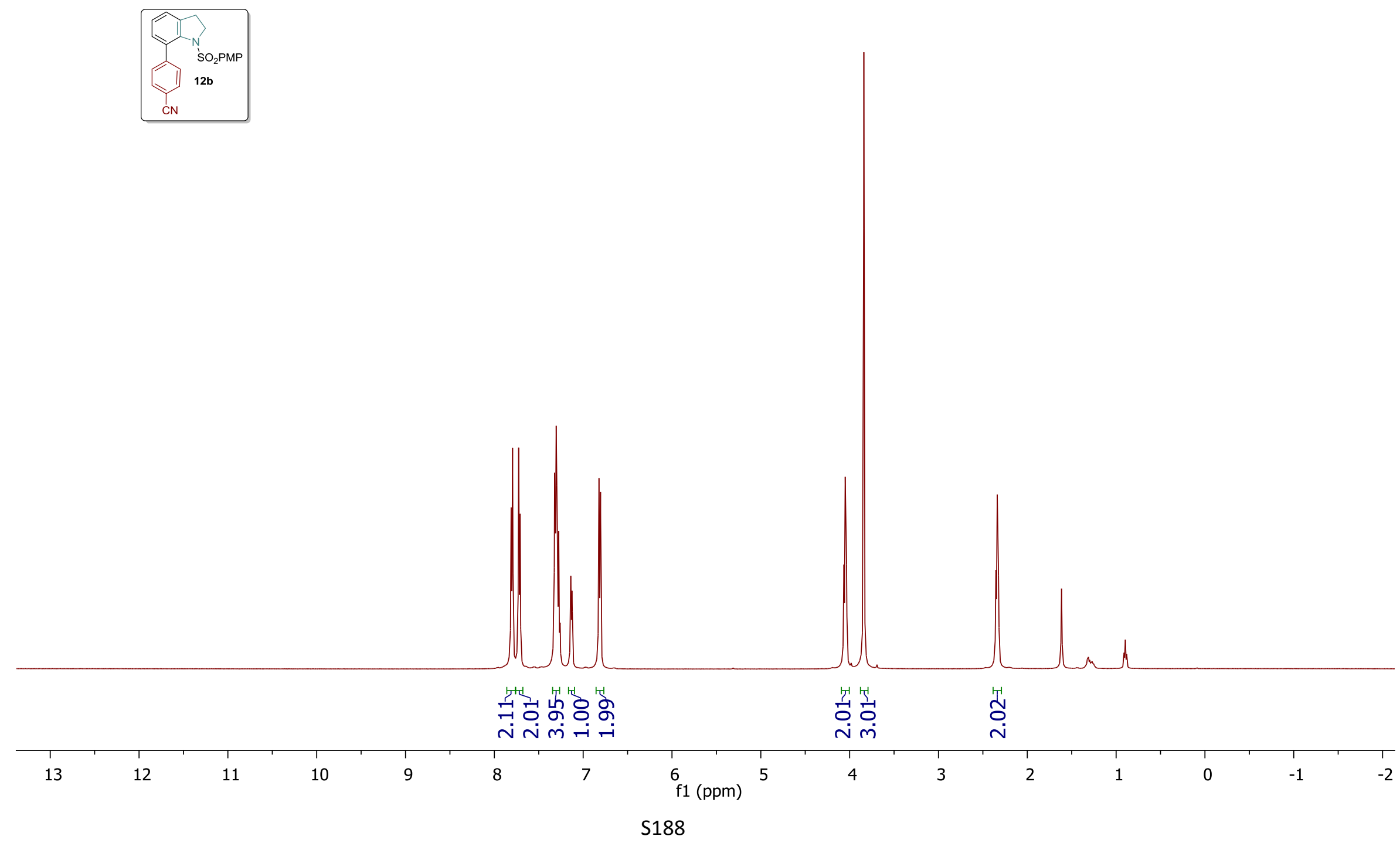




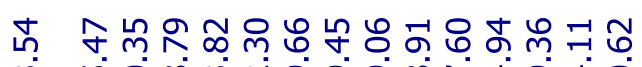

兽 守守兽

근

เู่กิ่

กิ

I
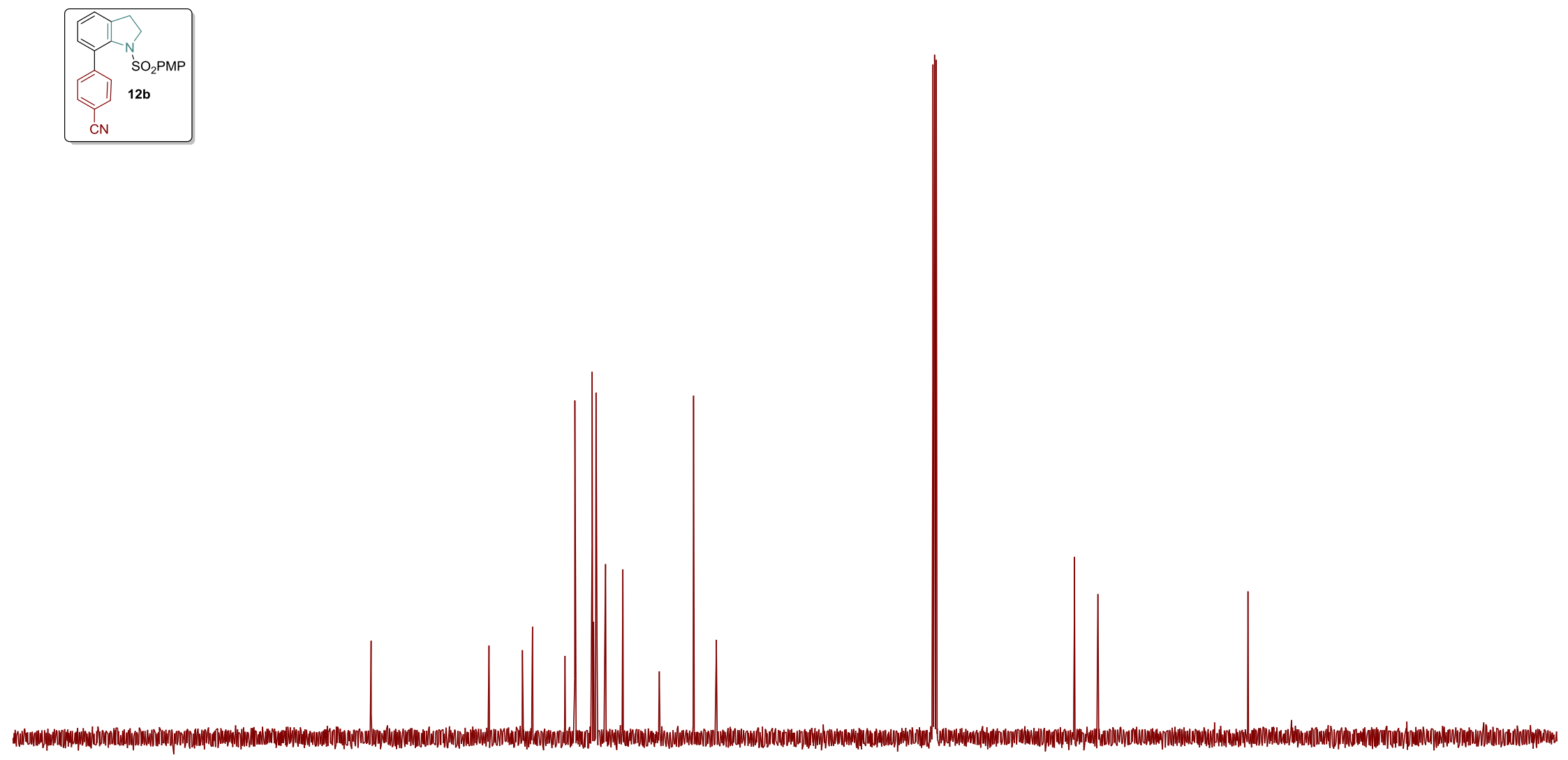

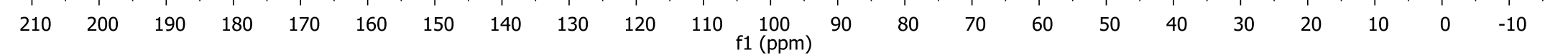

